\title{
Protective Particle Coatings applied by Cold Plasma Spraying
}

\section{Dissertation}

to acquire the doctoral degree in mathematics and natural science 'Doctor rerum naturalium' at the Georg-August-Universität Göttingen

in the doctoral degree program 'Material Science Wood' at the Georg-August University School of Science (GAUSS)

Submitted by

Lena Marie Wallenhorst

from Bremen

Göttingen, 2017 


\section{Thesis Committee}

Professor Dr. Gisela Ohms, Laboratory of Analytical Measurement Techniques, HAWK University of Applied Sciences and Arts

Professor Dr. Wolfgang Viöl, Laboratory of Laser and Plasma Technologies, HAWK University of Applied Sciences and Arts

Professor Dr. Holger Militz, Wood Biology and Wood Products, Faculty of Forest Sciences, Georg-August-University Göttingen

\section{Members of the examination board}

Referee:

Professor Dr. Gisela Ohms, Laboratory of Analytical Measurement Techniques, HAWK University of Applied Sciences and Arts

Co-referee:

Professor Dr. Wolfgang Viöl, Laboratory of Laser and Plasma Technologies, HAWK University of Applied Sciences and Arts

Co-referee:

Professor Dr. Holger Militz, Wood Biology and Wood Products, Faculty of Forest Sciences, Georg-August-University Göttingen

Other members of the examination board:

Professor Dr. Stephan Wieneke, Laboratory of Laser and Plasma Technologies, HAWK University of Applied Sciences and Arts

Professor Dr. Carsten Mai, Wood Biology and Wood Products, Faculty of Forest Sciences, Georg-August-University Göttingen

Professor Dr. Cynthia Volkert, Institute of Materials Physics, Georg-August-University Göttingen

\section{Date of oral examination:}

18.12.2017 


\section{Acknowledgements}

Many people have enriched my years as a $\mathrm{PhD}$ student through their professional advice and friendship. I would like to take this opportunity to express my gratitude towards some of those who indirectly contributed to the completion of this thesis.

First of all, I cordially thank my supervisors Prof. Gisela Ohms, Prof. Wolfgang Viöl and Prof. Holger Militz. I am very thankful for the opportunity to work on this fascinating topic as well as for their guidance and support.

Many thanks also to my colleagues from the Laboratory for Laser and Plasma Technology. I particularly thank Georg for his advice, criticism, support and fruitful discussions. I am further much indebted to various people who helped me with technical issues, especially Nils, Martin B., Martin K., Enrico, Chris O., Viktor, and Richard. Deike and Mirco, thank you for your help with chemical issues. Moreover, I thank my office-mates Ghiath and Roman for the great atmosphere in our office, countless tea-sessions and particularly for their support during these intense months towards the end of my PhD. Jan and Jenny: I will miss our cosy lunch breaks.

I further thank Roger and Chris G. for teaching me a lot about the English language and the art of writing research articles. Thank you, Roger, also for proofreading this thesis.

And last, but definitely not least, my most sincere thanks to my wonderful family, including my companion, for their wholehearted support and sympathy. 


\section{Abstract}

In this thesis, two different types of particle coatings deposited by cold plasma spraying on wood and glass substrates were investigated in an attempt to achieve protection of wood or other surfaces, particularly in outdoor applications. The first type involved coatings comprised of zinc and zinc oxide that were deposited from elemental zinc. Zinc oxide features several properties favourable to wood protection, such as absorption of ultraviolet light. Indeed, UVblocking properties of semi-transparent coatings on acrylic glass were confirmed and UVinduced discolouration of wood could be distinctly reduced by coatings with increased particle concentration. Coating systems comprising a $\mathrm{Zn} / \mathrm{ZnO}$ particle coating and a polyurethane (PUR) top coat were found to decrease photodiscolouration without damage to the PUR matrix by photocatalytic decomposition. The second type of coating involved the deposition of a powder made of poly(methyl methacrylate) (PMMA), reinforced with aluminium trihydrate $\left(\mathrm{ATH}\right.$ or $\left.\mathrm{Al}(\mathrm{OH})_{3}\right)$ filler particles. This powder constitutes an industrial waste of a bulk material that has properties usable in wood protection; hence, its deposition was studied in order to explore a method of recycling the powder to make use of the material's protective potential. The deposition of pure PMMA/ATH powder yielded coatings with insufficient abrasion resistance, but their characterisation confirmed a certain potential to bond well, particularly to polar matrix materials. Particle fixation was achieved by the addition of a phenol-formaldehyde (PF) powder to the PMMA/ATH raw material. Studies on the coating's morphology and chemical composition suggest a film formation of the reacted PF which encased the PMMA/ATH particles and the generation of covalent bonds between the layer and the substrates as adhesion mechanisms. 


\section{Zusammenfassung}

Diese Arbeit stellt Studien zur Abscheidung und Analyse zweier unterschiedlicher Partikelschichten vor, die mittels Kaltplasmasprühen auf Holz- und Glassubstrate aufgebracht wurden. Primäres Ziel war dabei der Schutz von Holz und anderen Materialien vor Schädigungen bei Anwendung im Außenbereich. Auf diese Weise wurden zunächst Schichten bestehend aus Zink und Zinkoxid abgeschieden, da Zinkoxid über diverse Eigenschaften wie beispielsweise die Absorption von ultraviolettem Licht verfügt, welche nützlich im Bereich des Holzschutzes sind. Tatsächlich ließ sich für semitransparente Schichten auf Acrylglas eine reduzierte Transmission im UV-Bereich nachweisen. Weiterhin waren Schichten mit erhöhter Partikelkonzentration in der Lage, die durch UV-Licht induzierte Verfärbung von Holz zu mindern. Ebenso konnten Schichtsysteme auf Basis einer entsprechenden Partikelschicht und einer Polyurethanversiegelung (PUR) die Verfärbungen durch UV-Licht stark reduzieren, ohne dabei die PUR-Matrix durch photokatalytischen Abbau zu schädigen. Als zweites Ausgangsmaterial wurde ein Pulver aus Polymethylmethacrylat (PMMA) genutzt, das mit Aluminiumtrihydrat (ATH oder $\mathrm{Al}(\mathrm{OH})_{3}$ ) angereichert war. Dieses Pulver fällt als industrieller Abfall bei der Bearbeitung eines Vollmaterials an, welches auf Grund seiner Eigenschaften auf vielfältige Weise Verwendung im Bereich des Holzschutzes finden könnte. Daher wurde dieses Pulver sowohl auf Holz- als auch auf Glasoberflächen appliziert, um Recyclingoptionen unter Berücksichtigung der protektiven Eigenschaften zu untersuchen. Diese Studien ergaben, dass die Abscheidung von reinem PMMA/ATH Pulver zu Schichten mit unzureichender Abriebfestigkeit führt. Allerdings konnte durch deren Charakterisierung eine prinzipielle Eignung zum Anbinden des Schichtmaterials an polare Matrixmaterialien nachgewiesen werden. Eine mechanische Stabilisierung ließ sich durch Beimischung eines Phenol-Formaldehydpulvers zum PMMA/ATH Rohmaterial erreichen. Studien über die morphologischen und chemischen Eigenschaften solcher Schichten deuten auf eine Umhüllung der PMMA/ATH-Partikel mit reagiertem PF sowie auf die Erzeugung kovalenter Bindungen zwischen der Schicht und den Substraten hin. 


\section{List of Publications}

This thesis is based on the following research articles, which are referred to by their Roman numerals:

I L. M. Wallenhorst, L. Loewenthal, G. Avramidis, C. Gerhard, H. Militz, G. Ohms, W. Viöl: Topographic, optical and chemical properties of zinc particle coatings deposited by means of atmospheric pressure plasma. Applied Surface Science 410 (2017), pp. 485-493.

II L. Wallenhorst, L. Gurău, A. Gellerich, H. Militz, G. Ohms, W. Viöl: UV-blocking properties of $\mathrm{Zn} / \mathrm{ZnO}$ coatings on wood deposited by cold plasma spraying. Applied Surface Science 434 (2018), pp. 1183-1192.

III L. M. Wallenhorst, S. Dahle, M. Vovk, L. Wurlitzer, L. Loewenthal, N. Mainusch, C. Gerhard, W. Viöl: Characterisation of PMMA/ATH layers realised by means of atmospheric pressure plasma. Advances in Condensed Matter Physics (2015).

IV L. Wallenhorst, R. Rerich, M. Vovk, S. Dahle, H. Militz, G. Ohms, W. Viöl: Enhancing the abrasion resistance of PMMA/ATH layers realised by means of atmospheric pressure plasma powder deposition on wood. Proceedings of the $11^{\text {th }}$ International Conference "Wood Science and Engineering in the third Millenium", Braşov (Romania, 2017).

V L. Wallenhorst, R. Rerich, M. Vovk, S. Dahle, H. Militz, G. Ohms, W. Viöl: Morphologic and chemical properties of PMMA/ATH layers with enhanced abrasion resistance realised by cold plasma spraying at atmospheric pressure. Advances in Condensed Matter Physics (2018). 


\section{Abbreviations}

AFM

ATH

ATR

CWRA

DBD

EDX

FTIR

HMDSO

HMTA

ICP

LSM

LFS

MIES

PDMSO

$\mathrm{PFH}$

PMMA

PUR

$\mathrm{RH}$

ROS

SEM

TEOS

UPS

UV

VOC

WCA

XPS
Atomic Force Microscopy

Aluminium Trihydrate $\left(\mathrm{Al}(\mathrm{OH})_{3}\right)$

Attenuated Total Reflection

Constant Wetting Rate Angle

Dielectric Barrier Discharge

Energy Dispersive X-Ray Spectroscopy

Fourier Transform Infrared Spectroscopy

Hexamethyldisiloxane

Hexamethylenetetramine

Inductively Coupled Plasma

Laser Scanning Microscopy

Liquid Flame Spray

Metastable Impact Electron Spectroscopy

Polydimethylsiloxane

Perfluorohexane

Poly(methyl methacrylate)

Polyurethane

Relative Humidity

Reactive Oxygen Species

Scanning Electron Microscopy

Tetraethyl Orthosilicate

Ultraviolet Photoelectron Spectroscopy

Ultraviolet

Volatile Organic Compound

Water Contact Angle

X-Ray Photoelectron Spectroscopy 


\section{Contents}

1. Introduction $\quad 1$

2. State of the Art and Research 3

2.1 Plasma treatment of wood and wood products .............................................. 3

2.1.1 Pre-treatments using plasmas from oxidising gases........................... 4

2.1.2 Deposition of protective layers using plasma processes .......................5

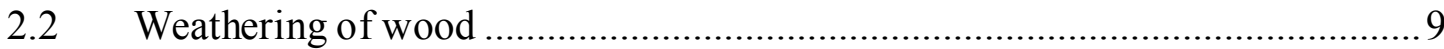

2.2.1 Photodegradation and photostabilisation of wood ............................ 9

2.2.2 Fungal infestation of wood.......................................................... 13

2.3 Characteristics and properties of zinc oxide............................................... 14

2.4 Characteristics and properties of poly(methyl methacrylate)/ aluminium

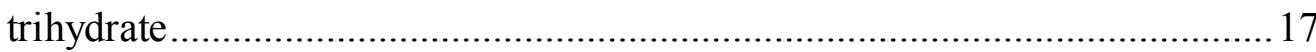

3. Experimental Setup and Methods 20

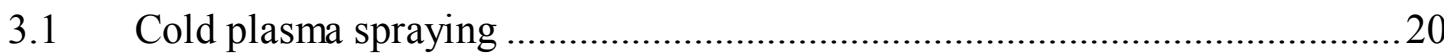

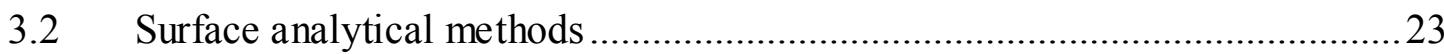

3.2.1 Methods to investigate morphologic properties..............................23

3.2.2 Methods to investigate chemical properties....................................2 24

4. Deposition of Zinc/Zinc Oxide Particles 28

4.1 Characterisation of layers deposited on glass substrates ..............................28

4.2 Exposure of zinc/zinc oxide-coated wood to blue stain ...............................29

4.3 Exposure of zinc/zinc oxide-coated wood to UV light...................................32

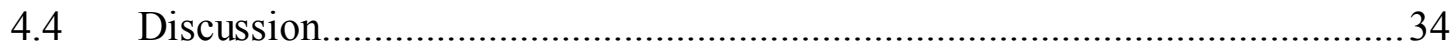

5. Deposition of Poly(methyl methacrylate)/Aluminium Trihydrate Particles 37

5.1 Characterisation of pure poly(methyl methacrylate)/aluminium trihydrate coatings on wood 
5.2 Enhanced abrasion resistance of poly(methyl methacrylate)/aluminium trihydrate coatings on wood and glass substrates........................................ 39

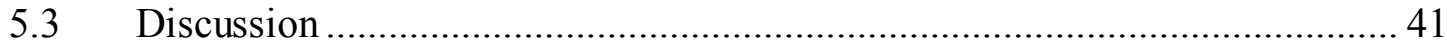

6. Summary and Outlook $\quad 44$

Appendix - Hydrophobic Plasma Polymers 47

$\begin{array}{ll}\text { References } & 53\end{array}$

Publications

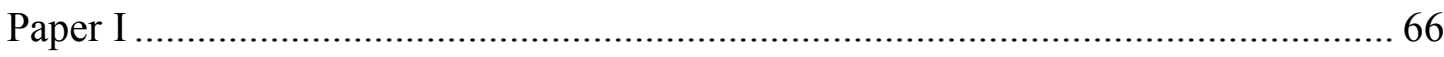

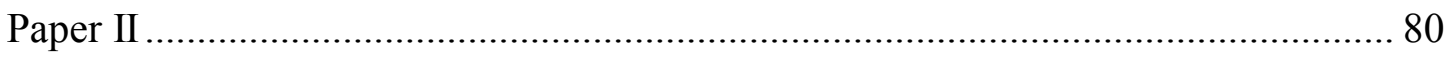

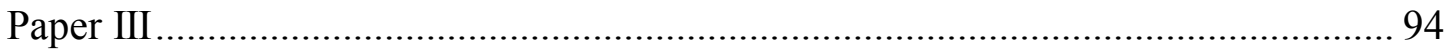

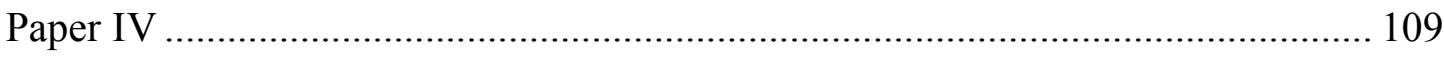

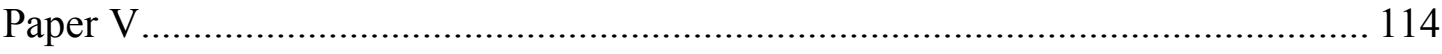





\section{Introduction}

Every material used in exterior applications is subjected to environmental degradation. Depending on the specific matter and its surrounding conditions, different types of protection are needed to slow down the complex physico-chemical changes leading to deterioration. Particularly affected by degradation in outdoor use are timber surfaces. Wood represents one of the oldest and most versatile materials used, for example, for the construction of buildings or furniture. It is a renewable and sustainable resource featuring outstanding properties such as a low thermal expansion and a high mechanical strength together with a low density [1]. Wood still promises remarkable innovative potential. Most temperate wood species, however, need ample protection when used outdoors. Various biotic and abiotic factors contribute to the degradation of wood, including attacks by microorganisms or insects, changes in humidity and temperature, exposure to sunlight, and possibly fire [2-9]. Since the studies presented in this thesis predominantly aim to increase the longevity of wood, the term "protective" therefore refers to the kind of protection needed to shelter wood in exterior use.

Some tropical wood species feature a higher resistance to weathering; however, a limited availability and growing concerns about sustainability of the production process have led to a reduction of their use. In addition, restrictions have been imposed on several effective wood preservatives due to their toxic potential [10,11]. Therefore, the search for effective but safe protective agents for timber materials still plays a major role in contemporary research. Among the promising approaches to enhance protection are different types of plasma treatments. Technical plasmas are commonly applied to tailor the surface properties of various materials like polymers or glasses. In wood science, the use of plasmas comprising oxidative species is being widely investigated in order to increase wettability and thus improve adhesion or liquid uptake [12-17]. In this way, the application of conventional preservatives may be improved to enhance protection.

Moreover, plasma technology affords the outstanding possibility to introduce reactive and film-forming species into a plasma discharge. In this manner, a direct generation of layers can be achieved, for instance, by plasma polymerisation [18]. Many of these approaches aim at the creation of hydrophobic coatings to reduce water and moisture uptake [19-24]. Moreover, particles can be introduced in a plasma, for example in plasma spraying processes to melt the particles and subsequently propel them towards a surface where they solidify and adhere through micromechanical clamping [25,26]. However, conventional plasma spraying involves high substrate temperatures and is therefore only suitable for materials featuring a sufficient 
heat resistance. Wood, in contrast, already starts decaying at temperatures above $130{ }^{\circ} \mathrm{C}[7]$. Recent studies have reported the application of a modified plasma spraying process, namely cold plasma spraying, which maintains low substrate temperatures and therefore enables the coating of thermosensitive materials like wood [27-30].

Various powder materials are eligible as the base material for a deposition by cold plasma spraying. Since the targeted application of the coatings studied throughout this work is the protection of wood, one type of coating containing zinc oxide was investigated. $\mathrm{ZnO}$ features several properties favourable to wood protection. It interacts with ultraviolet light and can therefore be used to diminish damage from exposure to sunlight [31]. Moreover, it manifests a biocidal action against microorganisms like fungi or bacteria [32]. Hence, coatings comprising zinc oxide may significantly reduce the degradation of wood used outdoors. Besides the characterisation of such coatings on wood and glass substrates, the focus of this work is on reducing the photodegradation and fungal infestation of wood.

In addition, a second type of coating comprised of poly(methyl methacrylate) reinforced with aluminium trihydrate (PMMA/ATH) was investigated. Here, the starting point was the search for recycling options for a powder that accrues as industrial waste in order to ensure a more complete utilisation of a valuable material and improve sustainability. The PMMA/ATH bulk material stands out due to its mechanical properties (e.g. high stiffness, wear and heat resistance), chemical resistance, and flame-retardant as well as smoke-suppressing effect [33,34]. If some of these properties can be transferred to coatings made of the PMMA/ATH waste powder, it would ensure an effective protection of wood or other materials and simultaneously provide a cost-effective and adequate way to recycle an industrial waste. Since the coatings applied by the use of pure PMMA/ATH powder did not exhibit a sufficient abrasion resistance, the focus was on ensuring particle fixation.

Hence, the objective of this work was to deposit suitable protective substances by cold plasma spraying on wood and other substrates, and to characterise the resulting coatings including an evaluation of their protective potential. Moreover, an approach to ensure the recycling of PMMA/ATH waste powder was explored. To avoid unintentional release of zinc or aluminium compounds to the environment and particularly to aquatic systems, methods to ensure particle fixation were sounded out. 


\section{State of the Art and Research}

This chapter reviews important background information and presents advances in current research. First, recent progress in the plasma treatment of wood both to increase and reduce wettability are discussed. To understand the need for UV protection of wooden surfaces, some aspects of the chemical composition of wood together with photodegradation mechanisms are presented. Moreover, fungal infestation of wood is reviewed. Finally, the used coating materials are presented along with their characteristics and challenges when used as layers.

\subsection{Plasma treatment of wood and wood products}

Plasma treatments are increasingly gaining interest as a method to modify wood and wood products. A plasma in the physical sense denotes a (partially) ionised gas consisting of free electrons, ions, radicals, and neutral particles of alternating excitation levels permanently interacting among each other and with photons. Depending on the type of plasma, the constituents possess certain energies and may interact with surfaces in manifold ways. Plasmas can be generated at low, high and atmospheric pressure. Since large-scale treatments are usually required to modify wood, the focus of this work is on atmospheric-pressure plasmas. A plasma at atmospheric pressure for technical use is often generated by applying high voltage to some arrangement of electrodes. Omnipresent electrons are accelerated and may interact with atoms or molecules via elastic or inelastic scattering. Inelastic processes can lead to excited states or ionisation and therefore to generating additional free electrons which eventually yield an electron avalanche (“electrical breakdown"). Moreover, the scattering may cause dissociation and therefore generate reactive molecular fragments. Details about the generation and properties of plasmas can be found in the literature [35-38].

Important characteristics of a plasma are its temperatures. In some plasmas (thermal plasmas), the electrons feature the same temperature as the heavier particles (ions, neutral particles) and are therefore in thermal equilibrium. For "cold plasmas" (non-thermal plasmas), however, the constituents can exhibit very different temperatures. Particles of a higher mass require more energy to be accelerated and therefore cannot achieve high temperatures in the plasma. Consequently, the energy transfer upon collision with a substrate (heating via scattering) remains low and therefore prevents heating to some extent. Samples may even remain at room temperature. On the other hand, light electrons can easily be accelerated by external electromagnetic fields. These particles subsequently possess sufficient energy to interact with 
atoms or molecules via inelastic scattering and can therefore initiate manifold chemical processes. For example, Peters et al. [39] thoroughly studied various temperatures occurring in three different dielectric barrier discharges ${ }^{1}$. Despite a mean electron temperature of up to $70,000^{\circ} \mathrm{C}$, the surface temperature did not exceed $42.3^{\circ} \mathrm{C}$ during $30 \mathrm{~s}$ of air plasma treatment. The treatments distinctly increased the surface free energy of all treated materials. Hence, plasma treatments are a versatile and effective tool to alter the surface properties of various materials.

\subsubsection{Pre-treatments using plasmas from oxidising gases}

Plasmas containing oxygen species proved to be particularly interesting for pre-treatments of wood and wood products to enhance subsequent processing like bonding or painting, especially with waterborne media. Various researchers were able to confirm the positive effects on wettability, liquid uptake, bonding, and adhesion of paints [14,15,41-46]. In many cases, ambient air at atmospheric pressure is feasible as process gas; hence, the implementation of expensive and size-limiting vacuum setups or costly technical gases can be avoided. These treatments are therefore generally suitable for large-scale and in-line processing. When exposed to such a plasma, several effects were found to occur, which may increase the surface's wettability and improve the spreading, bonding, or even uptake of modification agents. These effects can be summarised as follows:

- removal and oxidation of hydrophobic wood extractives,

- oxidation of wood constituents/accumulation of polar functional groups,

- $\quad$ shift of $\mathrm{pH}-\mathrm{level}$ towards more acidic values ${ }^{2}$,

- differential etching leading to an increased surface roughness.

During ageing of wood, wood extractives from the bulk material migrate towards the surface and create a rather hydrophobic layer ${ }^{3}$. In conventional processing, such layers are removed by sanding. Wolkenhauer et al. [41], however, were able to show that the increase in wettability by plasma treatments can be superior to sanding. During certain plasma treatments, extractives are efficiently degraded and, to a minor degree, oxidised [47-49]. In this way, the rather hydrophilic wood bulk material is exposed - comparable to a surface after

1 A dielectric barrier discharge contains at least one insulating layer between the electrodes. This type of discharge is particularly suitable for generating cold plasmas at atmospheric pressure for many (industrial) applications [40].

2 This shift may affect adhesion in a positive but also negative way, depending on the type of adhesive/paint.

3 The nature of wood extractives will be reviewed in Chapter 2.2.1. 
sanding. Moreover, studies by XPS and FTIR revealed an oxidation of wood constituents during plasma treatment, leading to an accumulation of polar functional groups $[16,48,50$ 52]. Odrášková et al. [51] confirmed the generation of polar groups and a shift of the pH-level to lower, more acidic, values. Klarhöfer et al.[50] used a combination of XPS, UPS and $\mathrm{MIES}^{4}$ to study the chemical effects of plasma treatments on lignin and model substances for lignin (coniferyl alcohol) and for cellulose (cellubiose, glucose and methanol). Their results suggest a degradation of both lignin and cellulose but at different rate. In addition, lignin is oxidised in a plasma generated from synthetic air, leading to the formation of hydroxyl $(-\mathrm{OH})$, carbonyl $(-\mathrm{C}=\mathrm{O})$ and carboxyl $(-\mathrm{C}(=\mathrm{O}) \mathrm{OH})$ groups. A generation of carbonyl groups could also be shown for the cellulose model substances; however, cellulose appeared to be degraded through a depletion of hydroxyl groups.

Moreover, etching of the surface takes place, leading to an increased surface roughness and hence a higher effective surface. Jamali and Evans [53] used scanning electron microscopy (SEM) and chromatic confocal profilometry to study differential plasma etching of wooden surfaces. They discovered that the parts of wood cells exhibiting a dominant contribution from lignin or crystalline cellulose were affected less by water vapour plasma treatment than those parts featuring a high contribution from amorphous cellulose.

Even though plasma treatments are known to affect only the uppermost surface with a penetration depth of approximately some tens or hundreds of nanometres [54], there is evidence that electrical breakdown can also occur to some extent within the bulk material's cavities and thus even lead to an improved uptake of liquid modification agents [55].

\subsubsection{Deposition of protective layers using plasma processes}

In contrast to the use of plasmas as pre-treatment to enhance further processing, it is also possible to introduce reactive substances into a plasma and directly deposit a layer on (wooden) surfaces. Plasma polymerisation permits, for example, the generation of highly hydrophobic coatings. Wood is particularly affected by changes in humidity leading to cracks in the material due to alternating swelling and shrinking. Furthermore, a sufficient water content is prerequisite for bacteria and fungi growth [56]. Many techniques are therefore

$4 \quad$ UPS: Ultraviolet Photoelectron Spectroscopy, MIES: Metastable Impact Electron Spectroscopy. This combination of spectroscopic techniques permits a surface-sensitive differentiation between hydroxyl and carbonyl groups as well as between conjugated and non-conjugated carbon bonds. The information depth should not exceed $10 \mathrm{~nm}$ [50]. 
applied or studied to inhibit water uptake, ranging from conventional paint to plasma-assisted coating.

Polymerisation in the conventional way needs special, low molecular monomers. After initiation, these molecules form long polymer chains. However, this process often requires high temperatures which would destroy wood. Furthermore, a polymer created in this way usually demands additional processing steps to bond it to a surface. When introducing such monomers into a plasma, some energetic species may initiate polymerisation in a conventional sense (plasma-induced polymerisation). Contrary to the conventional polymerisation, however, plasma polymerisation also occurs on an atomic level through plasma-state polymerisation. In this process, the precursor molecules (outgoing material) may dissociate to reactive fragments in a very efficient way. These fragments recombine to three dimensional, highly branched clusters, condense on the substrate's surface and continue growing. On the other hand, simultaneous etching takes place. In summary, the following processes occur competitively during polymerisation in a plasma $[18,57-59]$ :

- plasma-induced polymerisation,

- plasma-state polymerisation,

- plasma etching.

The predominating effect is determined by the specific plasma conditions. Plasma-induced polymerisation is a chain reaction polymerisation on the molecular level. This process may dominate particularly at very low power input and resembles conventional polymerisation. In contrast, plasma-state polymerisation occurs on the atomic level from reactive intermediate species created in the plasma. Concurrently with plasma etching, this process can dominate at medium power input. By plasma etching, the substrate's surface and the previously deposited polymer are etched. This process may dominate at very high power levels. Plasma etching also accounts for the fragmentation of monomer molecules [59].

In many cases, the resulting plasma polymers are found to be highly crosslinked and branched, and therefore exhibit a comparatively high mechanical, chemical and thermal stability. Thin films with thicknesses in the nanometre range, low pinhole density and a very good adhesion to the substrate may be achievable in a single-step process. Nearly all organic compounds, even those being saturated, can be polymerised in a suitable plasma process $[18,57-59]$.

Precursors commonly used in research to deposit hydrophobic coatings on wood can be sorted in three different classes: hydrocarbons like ethylene $\left(\mathrm{C}_{2} \mathrm{H}_{4}\right)$ and acetylene $\left(\mathrm{C}_{2} \mathrm{H}_{2}\right)$, fluorine- 
containing compounds such as tetraflouromethane $\left(\mathrm{CF}_{4}\right)$ and hexafluoropropylene $\left(\mathrm{C}_{3} \mathrm{~F}_{6}\right)$, and organosilicons like hexamethyldisiloxane (HMDSO or $\mathrm{O}\left[\mathrm{Si}\left(\mathrm{CH}_{3}\right)_{3}\right]_{2}$ ) and tetraethyl orthosilicate (TEOS or $\left.\mathrm{Si}\left(\mathrm{OC}_{2} \mathrm{H}_{5}\right)_{4}\right)$. Particularly HMDSO-based plasma coatings are being studied intensely in current research [19,23,60-66]. The chemical composition of the resulting coatings strongly depends on the specific deposition conditions. Some researchers $[23,63,65]$ have almost completely removed organic parts of the precursor and obtained $\mathrm{SiO}_{2}$ coatings of high purity. On the other hand, the original structure may be more visible. Denes et al. [62] obtained layers mostly composed of oligomers or polymers with structural units based on $\mathrm{Si}\left(\mathrm{CH}_{3}\right)_{\mathrm{i}}, \mathrm{i}=1,2,3$, bonded with oxygen, e. g. fragments of the form $\mathrm{Si}\left(\mathrm{CH}_{3}\right)-\mathrm{O}-\mathrm{Si}\left(\mathrm{CH}_{3}\right)$. All research groups have found very high water contact angles of more than $100^{\circ}$ on the coated wood substrates. Levasseur et al. [60] applied a coating predominately composed of $\mathrm{Si}\left(\mathrm{CH}_{3}\right)_{\mathrm{i}^{-}}$ $\mathrm{O}-\mathrm{Si}\left(\mathrm{CH}_{3}\right)_{\mathrm{j}}$ fragments to wooden surfaces and exposed the samples to natural weathering. The water contact angle remained almost constant even during eight months of natural ageing. Bente et al. [20] investigated plasma coatings based on silane $\left(\mathrm{SiH}_{4}\right)$ as well as organic precursors (ethylene and methane, $\mathrm{CH}_{4}$ ). The silane coatings yielded the highest contact angles $\left(145^{\circ}\right)$; furthermore, studies on the absorption of water droplets did not show any absorption even after $9 \mathrm{~h}$. Likewise, the organic coatings successfully increased the contact angles as well as the absorption time from around $165 \mathrm{~s}$ for untreated wood to $6.7 \mathrm{~h}$ (methane) and $7.5 \mathrm{~h}$ (ethylene). Rehn and Viöl [67] conducted studies on plasma coatings based on organic precursors (methane and acetylene). Contact angles of up to $155^{\circ}$ could be achieved, and also the water uptake was dramatically decreased. Magalhães et al. [68] generated highly hydrophobic coatings from ethylene, acetylene, 1-butene $\left(\mathrm{C}_{4} \mathrm{H}_{8}\right)$ and vinyl acetate $\left(\mathrm{CH}_{3} \mathrm{CO}_{2} \mathrm{CHCH}_{2}\right)$, but studies on moisture absorption showed no significant difference. They concluded that their coating process failed to cover small capillaries and pores. This hypothesis was supported from SEM-EDX mappings for coatings based on TEOS, which were studied instead of the organic coatings because of their suitability for silicon EDX mapping.

Moreover, several fluorine-containing precursors were tested [15,19,21]. Hexafluoropropylene $\left(\mathrm{C}_{3} \mathrm{~F}_{6}\right)$ appeared to be a good candidate for hydrophobic coatings with contact angles of up to $145^{\circ}[19,21]$. In addition to studies of water contact angle measurements, Toriz et al.[21] studied the water uptake and found a strong decrease for coated wood samples.

Several recent studies have investigated the incorporation of inorganic substances like $\mathrm{TiO}_{2}$, $\mathrm{Ag}$ or $\mathrm{ZnO}$ in such hydrophobic plasma coatings. These materials are known to feature 
protective properties that may protect wood from degradation by UV light, fungal or bacterial attack, and could reduce possibly harmful emissions from wood or adhesives by photocatalytic degradation. Denes and Young[69] dispersed $\mathrm{ZnO}$ along with organic compounds $^{5}$ into PDMSO (polydimethylsiloxane or $\mathrm{CH}_{3}\left[\mathrm{Si}\left(\mathrm{CH}_{3}\right)_{2} \mathrm{O}\right]_{\mathrm{n}} \mathrm{Si}\left(\mathrm{CH}_{3}\right)$ ) and deposited the plasma polymer on pine wood. The resulting hydrophobic coating combined with additives was found to reduce chemical and mechanical changes due to weathering. Profili et al. $[23,65]$ and Gherardi et al. [63] introduced $\mathrm{ZnO}$ and/or $\mathrm{TiO}_{2}$ nanoparticles into a discharge which polymerised HMDSO, thus obtaining the corresponding nanocomposites incorporated into a hydrophobic $\mathrm{SiO}_{2}$ coating with very little organic moieties. Beier et al. [64] sprayed a silver nitrate solution into the discharge zone during polymerisation of HMDSO to include nanoparticles into a $\mathrm{SiO}_{\mathrm{x}}$ matrix and studied antibacterial effects. The coatings deposited on glass substrates strongly reduced the growth of Escherichia coli even after several washing cycles. Moghaddam et al. [66] successively deposited $\mathrm{TiO}_{2}$ nanoparticles by liquid flame spray (LFS) ${ }^{6}$ and a plasma polymer based on HMDSO or perfluorohexane ( $\mathrm{PFH}$ or $\mathrm{C}_{6} \mathrm{~F}_{14}$ ). The combination of these coatings led to water contact angles of up to $160^{\circ}$, being superior to contact angles on wood coated only with either $\mathrm{TiO}_{2}$ or HMDSO/PFH. Likewise, the water uptake strongly decreased, particularly for the combination of both layers.

Recently, micro-sized copper and aluminium particles were deposited in the cold plasma spraying process [27-29] which was also applied in this work (see Chapter 3.1). Even though copper particles did not reduce the photodegradation of lignin due to artificial or natural weathering, resistance to fungal infestation (blue stain, Aureobasidium pullulans) was increased [27,28]. Further improvement was achieved by a preceding vacuum pressure impregnation with siloxanes [29]. In addition, the combination of siloxane impregnation and copper coating proved to protect wood efficiently from termite attack. When termites were placed in a box containing only treated samples (no-choice test), they attacked the sample to a similar extent as the untreated samples; however, mortality strongly increased. Choice tests containing treated and untreated samples showed a clear preference for untreated samples. The same deposition process was used throughout this work but with a focus on coatings based on $\mathrm{Zn} / \mathrm{ZnO}$ as well as PMMA/ATH. Both materials feature outstanding properties that

5 Electromagnetic radiation absorbents: benzotriazole, 2-hydroxybenzophenone, phtalocyanine, graphite.

6 Liquid Flame Spray: Injection of a liquid precursor into a spray gun. Upon exciting the burner nozzle, the solution is atomised and forms small droplets. These droplets subsequently evaporate in the flame and further react to nanoparticles [70,71]. 
are favourable for various applications in wood protection (cf. Chapters 2.3 and 2.4, respectively).

\subsection{Weathering of wood}

The protection of wood used outdoors still poses challenges. Wood is particularly susceptible to environmental degradation by sunlight, alternating moisture and temperature, atmospheric pollutants, abrasion by windblown particles and biological degradation for example by fungi or bacteria [72,73]. Based on several review articles $[4,5,72,73]$, this chapter summarises important aspects of weathering with a focus on the degradation of the primary wood constituents by sunlight. Moreover, deterioration by fungal infestation is reviewed.

\subsubsection{Photodegradation and photostabilisation of wood}

Wood is primarily composed of polysaccharides (cellulose, hemicellulose), lignin and extractives $^{7}$. Exposure to sunlight affects all components; however, lignin was found to play a major role in the degradation and discolouration of wood. One reason is the elevated absorbance of UV light, which features energies sufficient to initiate degradation and depolymerisation. Absorption spectra of wood, lignin and cellulose are shown in Figure 1 [6]. Terrestrial sunlight only includes wavelengths above $295 \mathrm{~nm}$ [74] due to absorption processes by ozone in the atmosphere. The penetration depth of photoreactive light $(\lambda<420 \mathrm{~nm})$ varies for and even within every wood species, but should not exceed approximately $220 \mu \mathrm{m}$ [75].

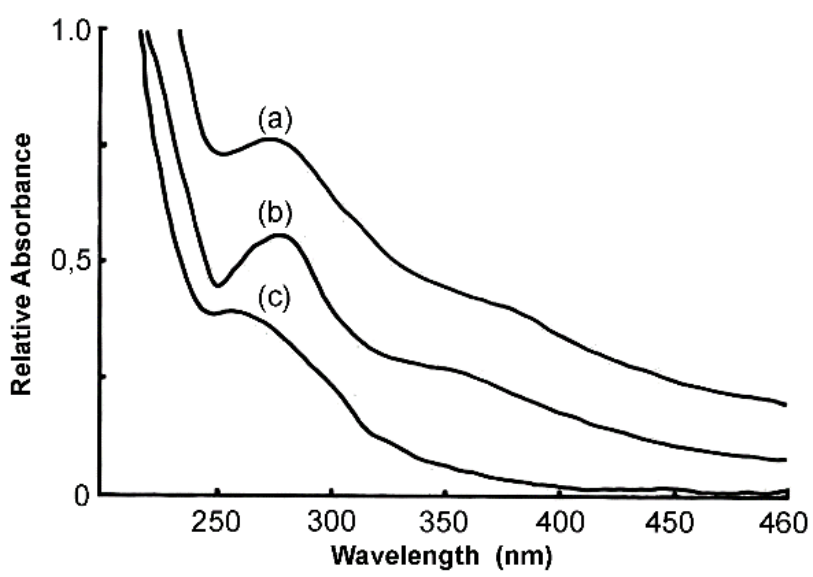

Figure 1: Absorbance of UV and violet light by (a) wood, (b) lignin, and (c) cellulose [6]. Terrestial sunlight is limited to $\lambda \geq 295 \mathbf{~ n m}$.

$7 \quad$ Inorganic ashes (up to $0.5 \%$ ) were not considered within this work. 


\section{Effects on polysaccharides}

Polysaccharides account for approximately two thirds of the wood mass and are almost exclusively represented by cellulose and hemicellulose ${ }^{8}$. These are essentially linear polymers without conjugated systems or carbonyl groups.

Cellulose chains are composed of $(1 \rightarrow 4)$ linked $\beta$-D-glucopyranose units $\left(\left[\mathrm{C}_{6} \mathrm{H}_{10} \mathrm{O}_{5}\right]_{\mathrm{n}}\right)$ with both crystalline and amorphous regions [5,76,77]. Cellulose contributes between $40 \%$ and $50 \%$ to the dry wood mass and is crucial for the mechanical properties of wood. Despite the reduced absorption of UV light compared to lignin, cellulose can undergo depolymerisation and thereby cause a loss in tensile strength [8]. However, the presence of lignin was found to stabilise cellulose by its strong absorption of UV light as well as by energy transfer from activated cellulose to lignin and thus deactivation of cellulose. Moreover, reactions in lignin seem to stabilise free radicals [78].

Hemicelluloses are smaller amorphous macromolecules and contain several different sugar monomers such as pentoses (arabinose, xylose) and hexoses (galactose, glucose, mannose) $[5,76,77]$. They were found to act as coupling agents between the polar surfaces of microfibrils and less polar lignin[79]. The specific composition differs between hardwood and softwood species. Hemicelluloses are also degraded by sunlight: leachates from weathered softwood include high rates of mannose and xylose [80].

\section{Effects on lignin}

Lignin is a highly complex, three-dimensional phenolic polymer. A definite structure to lignin does not exist due to the randomness of polymerisation processes; however, individual bond types have been studied intensely. Precursors of lignin are shown in Figure 2: (1) represents p-coumaryl alcohol which occurs as a minor precursor of both softwood and hardwood lignin, (2) depicts coniferyl alcohol which is the predominant precursor of softwood lignin, and (3) is sinapyl alcohol which, together with structure (2), is a precursor of hardwood lignin. These alcohols are linked by ether and carbon-carbon bonds [73,81,76,81]. Lignin is responsible for the cell wall's stiffness and for the bonding of wood cells in the middle lamella region [79].

$8 \quad$ Minor contributions arise e. g. from starch or pectin. 


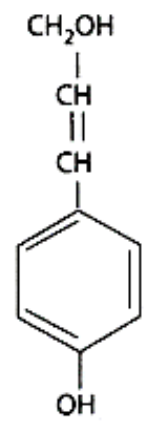

1

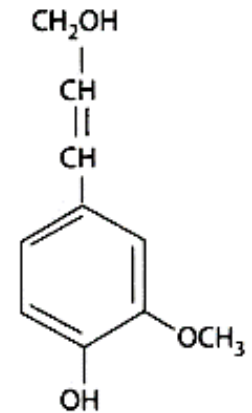

2

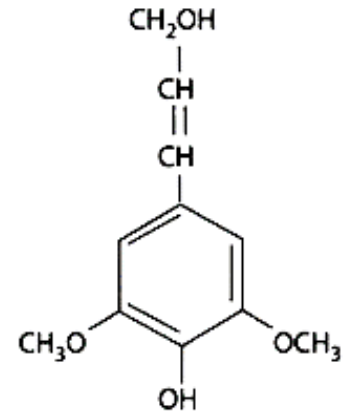

3

Figure 2: Lignin precursors. 1: p-coumaryl alcohol, 2: coniferyl alcohol, and 3: sinapyl alcohol [81,73].

Lignin represents the key structure in photodegradation. As mentioned earlier, it is a very good UV absorber. UV light features sufficient energy to dissociate parts of lignin involving carbonyl, biphenyl, or ring-conjugated double bonds [73]. In the presence of UV light and oxygen, different pathways give rise to the generation of free radicals - mainly phenoxy and ketyl radicals $[4,73,82]$. Free radical formation can be influenced by temperature, moisture, and the specific composition of the wood. Through further reactions with atmospheric oxygen, these radicals may eventually form new carbonyl groups such as non-conjugated aliphatic carbonyls, conjugated and aromatic carbonyls, and in particular chromophoric quinone structures, which account for the yellowing of wood upon UV irradiation [83]. The reaction products are water-soluble and can therefore be washed out by rain.

\section{Effects on extractives}

Extractives are usually low-molecular organic compounds that can, as the name implies, be extracted by using different solvents. Hundreds of different wood extractives have already been identified and depending on the wood species, their content may vary between approximately $0.5 \%$ and $20 \%$ [79]. The specific functions are not known for all types of extractives yet, but some extractives have been found to have a significant impact on the wood's colour, odour, and natural durability. The composition pattern of the extractives present in wood highly varies between different wood species and even between the sapwood and heartwood belonging to the same trunk. In sapwood, mainly glucose, fructose, sucrose, starch, fatty acids and only small amounts of polyphenols are present (primary extractives)[84]. Sugars and lipids can serve as energy storage compounds and therefore contribute to the physiological functions in the sapwood. Sapwood usually features a low amount of toxic extractives and is therefore susceptible to decay. On the other hand, when sapwood turns into heartwood, important changes affecting the nature and amount of 
extractives occur. For example, storage compounds are depleted and new extractives, e.g. polyphenols, are synthesised. In heartwood, mainly xylose, arabinose, mannose, resin acid, free $\mathrm{COOH}$, and large amounts of polyphenols are present (secondary extractives). Due to a generally higher amount of toxic extractives, the natural durability is usually higher than for sapwood [84,85]. Recent approaches have used selected extractives of durable wood species to enhance the longevity of timber from less durable species [5]. For example, resins can be used to improve water repellence. In general, a reduction of extractives by weathering may render the surface more hydrophilic [72]. Particularly some tropical wood species contain extractives which reduce photodegradation by acting as antioxidants and radical quenchers [5]. Extractives also contribute to the wood's colour. They can be photodegraded by UV light as well as by the visible portion of sunlight [73].

\section{Photostabilisation of wood}

Several types of photoprotective additives are currently being investigated to reduce damage from exposure to sunlight. Since photodegradation only occurs superficially, surface treatments are usually investigated.

A remarkable stabilisation may be achieved by additives based on chromic acid or chromated copper arsenate [72,73]; however, applicability is limited due to their toxicity. Another recent and promising approach uses wood extractives of durable species exhibiting high antioxidant capacities to impregnate less durable woods [86]. Diouf et al. [86] studied the impact of extractives from several wood species on poplar and pine wood. Extractives from padauk were found to ensure an effective protection, but also a local European wood, European walnut, reduced photodiscolouration.

Hindered amine light stabilisers (HALS) are derivatives of 2,2,6,6-tetramethyl piperidine and may also contribute to photostabilisation when used in a primer [5,73]. Studies on synthetic polymers (PP, PE) suggest that the stabilisation mechanism involves quenching of excited polymer-oxygen complexes as well as terminating free radicals [87].

Another important class of photoprotective additives are UV absorbers. Many organic absorbers usually based on benzophenone, benzotriazole, triazine, cinnamic derivatives, and oxalanilide have been studied successfully $[73,88]$. Moreover, inorganic UV absorbers such as rutile titanium dioxide, iron (III) oxide, or zinc oxide may provide effective protection from sunlight [31,89-92]. As semiconductors, these materials feature a band gap which enables the absorption of light with specific energies. For $\mathrm{TiO}_{2}, \mathrm{Fe}_{2} \mathrm{O}_{3}$ and $\mathrm{ZnO}$, this bandgap leads to the 
absorption of large parts of the solar spectrum, particularly in the UV range ${ }^{9}$. Moreover, elastic scattering of sunlight can enhance the photoprotective properties.

\subsubsection{Fungal infestation of wood}

When exposed to natural weathering, wooden surfaces undergo discolouration due to photodegradation of lignin and leaching by rain water. The resulting degradation products, however, may provide nutrition to fungi. The blue stain Aureobasidium pullulans (de Bary) Arnaud represents one of the most common fungi observed on weathered wood [73]. Since this fungus is only a staining fungus, it contributes to discolouration but does not distinctly affect wood stability[56]. However, the discolouration penetrates deep into the timber material and therefore cannot be removed by surfacing. A pullulans withstands even severe conditions such as temperatures above $80^{\circ} \mathrm{C}$, long periods without moisture, and can grow in a huge range of $\mathrm{pH}$ level (1.9-10.1) [73,93,94]. It mainly metabolises lignin breakdown products but is also capable of enzymatically degrading cellulose and hemicellulose to some extent $[73,94]$. Infestation by staining fungi usually requires a moisture content (occasionally) above $18 \%$ [95]; therefore, hydrophobic coatings may provide some protection.

In addition to staining fungi, wood decay fungi may also colonise surfaces. However, decay fungi usually attack living trees and stop growing after harvesting [56]. Decay fungi growth strongly depends on the surrounding conditions and requires the presence of free water in wood; hence, a moisture content above the fibre saturation point (around 30\%) is needed. Usually, such a high moisture content is rarely reached in timber, but staining fungi may increase hydrophilicity and absorption of water, and could therefore enable colonisation by decay fungi [56].

The effective protection of wood from biological attack and particularly from fungal infestation poses challenges. Preservatives of the first generation [10,96] mainly included creosotes, oil-borne pentachlorophenol, or water-borne arsenicals such as chromated copper arsenate. Despite a remarkable long-term effectiveness, their use is highly restricted in Europe or even prohibited due to strong concerns about health hazards imposed by the toxic or even potentially carcinogenic properties. Currently, most preservatives contain copper salts along with a cobiocide acting against copper-tolerant fungi such as alkaline copper quat ${ }^{10}$, copper azole, or copper xyligen. Problems arise due to leaching since copper may have negative 
impacts on aquatic systems $[10,96]$. Other formulations include boron compounds; however, since sufficient prevention of leaching has not yet been achieved, this type of preservative can only be used in the absence of rain and high moisture. Much research is currently being conducted on organic biocides such as triazoles, phenylsulfamides, carbamates, and many others $[10,96]$. Moreover, several approaches use inorganic micro- or nanoparticles to prevent biodeterioration. In particular, copper, zinc or silver particles are being studied intensely [2729,97-99].

\subsection{Characteristics and properties of zinc oxide}

One objective throughout this work was the deposition of coatings containing zinc oxide. Zinc oxide features a number of properties that are desirable in wood protection; therefore, the following chapter summarises several issues of importance for the targeted applications. Some aspects related to the thermal properties of zinc oxide are reviewed due to the relevance for the deposition method used in this work. The focus, however, is on the properties of $\mathrm{ZnO}$ as a semiconductor with a bandgap corresponding to the energy of light in the UV range. This feature leads to photoprotection but also to photocatalytic activity. Photocatalytic degradation may be used to reduce potentially harmful emissions (e. g. from wood or adhesives), but can also cause the degradation of organic matrix materials that are used to seal the particle coatings. It might even lead to a decomposition of the wood surface. Finally, the biocidal properties of zinc oxide are discussed.

\section{Aspects of thermal properties}

Zinc oxide exhibits a quite unusual behaviour when being heated. Unless high pressure is applied, it directly vaporises upon exceeding its melting point around $1975^{\circ} \mathrm{C}$ [100-102], mainly by dissociation into gaseous $\mathrm{Zn}$ and $\mathrm{O}_{2}$ [100,102]. A minor rate of sublimation and concurrent condensation has even been observed at temperatures as low as $500{ }^{\circ} \mathrm{C}[103]$. Throughout this work, a cold plasma spraying process as described below (Chapter 3.1) was applied to deposit coatings - a procedure which is principally based on a melting of the coating material and an acceleration towards the substrate's surface. To prevent damage to thermosensitive materials during the deposition process, temperatures need to be kept relatively low. Hence, a material like zinc oxide featuring a high melting point and such a complex thermal behaviour does not appear suitable as the base material in cold plasma spraying. Therefore, elemental zinc was used instead with a melting point around 
419. $6^{\circ} \mathrm{C}[104]$. Since the deposition proceeds under ambient conditions, sufficient oxidation was expected to occur ${ }^{11}$.

\section{Interaction with ultraviolet light}

Zinc oxide is a semiconductor with a wide direct bandgap around 3.2-3.4 eV for wurtzite $\mathrm{ZnO}^{12}$ [106-108], which corresponds to the energy of light with a wavelength of $365-387 \mathrm{~nm}$. Therefore, light featuring an energy that exceeds this band gap energy ${ }^{13}$ is absorbed by $\mathrm{ZnO}$. Photodegradation of wood is mainly initiated by the absorption of this UV light; therefore, $\mathrm{ZnO}$ is a promising candidate as a UV-protective coating on wood. The energy that is absorbed can subsequently be emitted as non-destructive heat; however, degradation processes of organic compounds may also be initiated. For several applications, this photocatalytic activity of $\mathrm{ZnO}$ and related materials is used to reduce potentially harmful emissions, for example volatile organic compounds (VOC) which occur in wood and adhesives. On the other hand, it needs to be kept in mind that the same process may also lead to a destruction of the wood surface or organic matrix materials that are applied as sealings to prevent (nano)particle release due to leaching or abrasion. The specific photocatalytic degradation processes are complex and greatly depend on the choice of the catalyst and the surrounding material. However, the basic photocatalytic mechanism can be described as follows [109-111]:

1. $M+S C a t \rightarrow M_{a d s}$

Adsorption of a reagent $M$ on the surface SCat of a catalyst (e.g. $\mathrm{ZnO}$ or $\mathrm{TiO}_{2}$ ). Possible reagents $M$ may be water or organic compounds.

2. $M_{a d s} \rightarrow M+S C a t$ Desorption of $M$ (no photocatalytic reaction occurs).

3. $S C a t+h v \rightarrow e_{c b}^{-}+h_{v b}^{+}$ Photoexcitation of the catalyst leading to a positive hole $h_{v b}^{+}$in the valence band and an electron $e_{c b}^{-}$in the conducting band.

4. $M_{a d s}+h_{v b}^{+} \rightarrow M_{a d s}^{+} \quad$ Formation of reactive radical state.

11

12 13

Detailed studies on the chemical composition are presented in Paper I.

Wurtzite crystal structure: stable phase under ambient conditions. Each anion (cation) is enclosed by four cations (anions) located at the corners of a tetrahedron [105].

That is, with a wavelength shorter than $365-387 \mathrm{~nm}$. 

5. $M_{a d s}^{+}+e_{c b}^{-} \rightarrow M_{a d s}$
Deactivation by recombination.
6. $M_{a d s}^{+} \rightarrow$ product + SCat
Chemical reactions yielding final product(s) and deexcitation of the catalyst.

Organic compounds can be decomposed directly by this mechanism[112]; however, the primary oxidising processes are assumed to be caused by radicals created from adsorbed water (formation of ${ }^{\circ} \mathrm{OH}$ radicals) and molecular oxygen (formation of ${ }^{\circ} \mathrm{O}_{2}$ radical anions) [111-113]. The specific processes crucially depend on the choice of the organic material but also on the catalyst; therefore, each system must be studied separately and the occurrence or rate of photocatalytic degradation may not be predictable. The predominant deexcitation pathway strongly depends on the availability of proper electron acceptors or donors [111]. For example, Liao et al. [114] found $\mathrm{TiO}_{2}$ to be superior to $\mathrm{ZnO}$ with regard to the photocatalytic decomposition of gaseous formaldehyde and suggested an elevated electron/hole recombination for the $\mathrm{ZnO}$ system.

Through photocatalytic processes, organic pollutants may be degraded on the one hand. As reaction products, mostly $\mathrm{CO}_{2}$ and $\mathrm{H}_{2} \mathrm{O}$ are assumed to occur $[109,112,115,116]$, but there is evidence that new and possibly harmful side products are generated [117]. On the other hand, this effect can decompose organic matrix materials that are used to seal particle coatings on wood surfaces. Due to the complexity of the specific processes, the occurrence or rate of degradation may differ strongly for various coating systems.

\section{Antimicrobial properties}

Another useful property of zinc oxide, particularly in its nanoparticulate form, is represented by its antibacterial and antifungal activity [97-99,118-122]. The antimicrobial mechanisms are still not entirely elucidated; however, several possible explanations are discussed in the literature [32]. For example, the generation of reactive oxygen species (ROS; mainly ${ }^{\circ} \mathrm{OH},{ }^{\circ} \mathrm{O}_{2}$, and $\mathrm{H}_{2} \mathrm{O}_{2}$ ) is assumed to play a major role. These ROS may, for example, lead to the destruction of cell membranes, lipids, DNA, and proteins, and eventually cause cell death $[32,120,123,124]$. ${ }^{\circ} \mathrm{O}_{2},{ }^{\circ} \mathrm{OH}$, and $\mathrm{H}_{2} \mathrm{O}_{2}$ are assumed to be formed predominantly from water and molecular oxygen in the photocatalytic processes as described above. However, there is evidence for significant ROS production even under darkness [32,125]. The underlying mechanism still needs to be determined. Moreover, the release of $\mathrm{Zn}^{2+}$ ions is assumed to be a second important mechanism $[32,119,124]$. These ions are assumed to play a role in the amino acid metabolism, enzyme system disruption, and active transport inhibition 
[32]. For example, McDevitt et al. [118] studied the effect of $\mathrm{Zn}^{2+}$ ions on Streptococcus pneumoniae and found an attachment to the manganese transport protein PsaA. By blocking these receptor sites, $\mathrm{Zn}^{2+}$ inhibits the manganese uptake which is essential for cellular processes.

In summary, zinc oxide is a promising candidate as a coating material to protect wood from degradation in outdoor use. $\mathrm{ZnO}$ absorbs light in the UV range and may therefore protect wood from photodegradation. Moreover, its antimicrobial properties should prevent damage induced by fungal or bacterial infestation. On the other hand, however, the possibility of photocatalytic degradation of organic materials must be kept in mind. Due to its complex behaviour during heating, zinc oxide was not chosen as the base material for application by cold plasma spraying. Elemental zinc was used instead, leading to a mixed system of zinc and zinc oxide (Paper I).

\subsection{Characteristics and properties of poly(methyl methacrylate)/ aluminium trihydrate}

Poly(methyl methacrylate) or PMMA that is reinforced with aluminium trihydrate ${ }^{14}$ (ATH) features several favourable properties. Within this work, waste PMMA/ATH powder was deposited on wood and glass substrates to work out a new recycling option that makes use of its protective potential. This chapter reviews important properties of the bulk material along with recent approaches to recycling the waste powder that accrues during post-polymerisation processing.

ATH (or $\left.\mathrm{Al}(\mathrm{OH})_{3}\right)$ as randomly distributed filler particles in a matrix of PMMA $\left(\left[\mathrm{C}_{5} \mathrm{O}_{2} \mathrm{H}_{8}\right]_{\mathrm{n}}\right)$ was first applied by Duggins et al.[33,126]. Various fabrication processes and specific compositions are now in use, but commonly applied formulations comprise an ATH content exceeding $50 \%$. The share of ATH may even be as high as $80 \%$ [127]. Usually, plate-type pre-products are produced during the polymerisation of PMMA. Since the material is thermoformable [33], it can easily be further processed to yield, for example, bathtubs or sinks $[33,128]$. The introduction of ATH filler particles in a PMMA matrix reduces production costs and simultaneously improves, for example, the material's stiffness, wear and heat resistance, and rheologic properties [33,34,129-131]. Moreover, it strongly reduces flammability through endothermal transformation to $\mathrm{Al}_{2} \mathrm{O}_{3}$ and water at high temperatures without releasing toxic gases [33,132,133]. Byrdy et al.[127] studied the influence of 
environmental factors on the flexural strength and modulus of elasticity of PMMA/ATH in natural weathering (study of facades) and under laboratory conditions. Even though high temperatures and moisture $\left(65^{\circ} \mathrm{C}\right.$ and almost $100 \% \mathrm{RH}$, respectively) did have an impact, only slight changes were observed upon UV irradiation for 3,000 hours at $40{ }^{\circ} \mathrm{C}$ and $50 \% \mathrm{RH}$ or 100 cycles of freezing and thawing. Therefore, PMMA/ATH is well-suited for an application in the building industry. Since it is also resistant to damage from many commonly used household acids, another application is the substitution of various indoor ceramics such as kitchen or bathroom countertops, sinks, and bath- or shower tubs [33,34].

However, much waste powder is generated during the manufacturing process by sanding, polishing or trimming. No adequate and cost-effective way of recycling the residual powder and chips has been found to date. Nevertheless, several approaches are discussed in the literature.

Hochberg and Young[134] proposed the application of waste PMMA/ATH powder in cleaning processes like the removal of paint from airplane skins. A blasting process using such powder should ensure a quick (due to the inorganic component) and gentle (due to the organic component) process. On the other hand, Kaminsky et al. [135] tried to recover monomer MMA from the waste by pyrolysis. Even though recovery of up to $97.2 \%$ MMA from pure PMMA is possible at a temperature of $450{ }^{\circ} \mathrm{C}$ [136], only up to $65 \%$ could be recovered from PMMA/ATH when the temperature is lowered to $400{ }^{\circ} \mathrm{C}$. The authors assume that the release of water from the decomposition of ATH along with the presence of chemical stabilisers account for the reduction in monomer gain.

A promising approach to recycle residual powder investigates its use as a filler for bitumen, particularly in asphalt mixtures [129,137-140]. Šušteršič et al. found PMMA/ATH to improve the viscoelastic and mechanical properties of bitumen at elevated temperatures occurring during handling and in service [129]. Further studies on pure bitumen [137] and asphalt mixtures [138] suggest a retardation of oxidation reactions in bitumen upon ageing ${ }^{15}$ and thus an increase in durability. Tušar et al. [139] conducted further field and laboratory studies on asphalt mixtures modified with PMMA/ATH waste powder and found, among other things, an improved dimensional stability in wheel tracking tests. Moreover, the water resistance was

15 In this context, the term "ageing" denotes the exposure to high temperatures in the presence of oxygen compounds. 
improved, presumably by promoting the adhesion between the aggregate and the binder (bitumen).

Within the frame of this thesis, PMMA/ATH powder was used to create coatings on wood and glass substrates by cold plasma spraying. The application of pure PMMA/ATH as the base material led to coatings without sufficient abrasion resistance. Therefore, the studies focused on the fixation and characterisation of the particle layers. If the bulk material's favourable properties can be transferred to the coatings, various applications for such layers on wood or other substrates are conceivable, including:

- fabrication of countertops for kitchens, bathrooms or laboratories with reduced production costs,

- flame-retardant coatings,

- weather-resistant coatings to protect, e. g., structural elements.

PMMA/ATH was found to be well-suited for an application by cold plasma spraying. However, the processes that occur during the deposition differ from those taking place for metal particles. No evidence was found for a melting (or rather: softening) in the effluent plasma and solidification on the surface; instead, the PMMA in PMMA/ATH powder appeared to be etched, thus uncovering ATH, and slightly oxidised (cf. Paper III) or significantly depolymerised (cf. Paper V), according to the specific deposition conditions. The possibility of $\mathrm{Al}(\mathrm{OH})_{3}$ reacting to $\mathrm{Al}_{2} \mathrm{O}_{3}$ and water upon heating has to be kept in mind. 


\section{Experimental Setup and Methods}

This chapter presents the setups and methods predominantly used throughout this work to generate and characterise different particle coatings. Cold plasma spraying is described in detail together with its advantages and challenges, particularly in distinction from other powder coating techniques such as conventional plasma spraying. Further, surface analytical methods to assess chemical and morphologic properties are specified.

\subsection{Cold plasma spraying}

Several different techniques to apply powder coatings on various surfaces have been developed during the past decades. However, many powder coating procedures face limitations because of the high substrate temperatures involved. For example, several powder coating techniques comprise a two-step process in which first the powder is applied, for example, by using an electrostatic gun ${ }^{16}$ followed by thermal curing. Depending on the melting point of the used powder material, the film formation may require high temperatures that would degrade thermosensitive materials like wood. Similar problems arise for thermal spraying $[25,26]$. Here, the coating material (usually metals) is melted in a high temperature region generated, for example, by a combustion gas flame (flame spraying) or an arc discharge (conventional plasma spraying), and subsequently propelled towards the sample to form solidifying splats, leading yet again to elevated substrate temperatures.

An emerging powder coating technique is represented by cold gas dynamic spraying (CGDS) [141-145]. In this technique, particles are highly accelerated to approx. 300$1200 \mathrm{~m} / \mathrm{s}$ by injection into a supersonic gas jet. When impinging on the substrate, the coating material undergoes plastic deformation to yield splats that adhere to the surface by micromechanical clamping. Even though this coating technique is still at a rather developmental stage, it seems to be well-suited for metal substrates. No literature about the application on wood surfaces was found, but results from CGDS on polymers suggest that the high particle velocity may lead to the erosion of more fragile surfaces [141-145]. Nonetheless, the application of CGDS to create powder coatings on wood seems to be another interesting approach to generating protective coatings.

16 An electrostatic gun charges the powder particles and sprays them towards the grounded substrate to achieve electroadhesion. 
The technique employed in these studies to create protective particle coatings is a modification of conventional thermal plasma spraying $[25,26,146]$. To apply coatings on wood and glass surfaces, a customised plasma jet coating system (Reinhausen Plasma GmbH) was used. The general setup is illustrated in Figure 3. As in conventional plasma spraying [146], the arc discharge is generated between a pen electrode (tungsten) and the surrounding nozzle-shaped copper electrode by a DC high voltage, streamed through by the process gas. Finally, the dry powder is injected into the effluent plasma and propelled towards the substrate. Typical arc discharges applied for plasma spraying feature high ampacities and high current densities and thus temperatures of several thousand centigrades [146]. Corresponding plasma jets are therefore not suitable for coating thermosensitive materials. In contrast, the arc discharge in cold plasma spraying is generated by a pulsed high voltage leading to a transient discharge (prevention of continuous electron flow) and consequently produces distinctly lower temperatures both in the discharge region and on the substrate ${ }^{17}$. Moreover, the use of small micro- or nanoparticles (large specific surface area) leads to short melting durations and therefore permits the application of a lower plasma power. Relatively high process gas flows can additionally be used, which also contributes to the cooling of the substrate. This specific deposition apparatus therefore avoids a significant thermal influence on the samples. Throughout these studies, the discharge was generated by applying an ignition voltage of approximately $15 \mathrm{kV}$ followed by an effective voltage of $2-3 \mathrm{kV}$. The maximum input power amounted to approximately $2 \mathrm{~kW}$. Pulses were generated at a repetition rate of $50 \mathrm{kHz}$ and a duration of $5-10 \mu \mathrm{s}$.

17 A proper characterisation of this specific plasma source is highly non-trivial and therefore addressed in separate current and future studies not related to this thesis. 


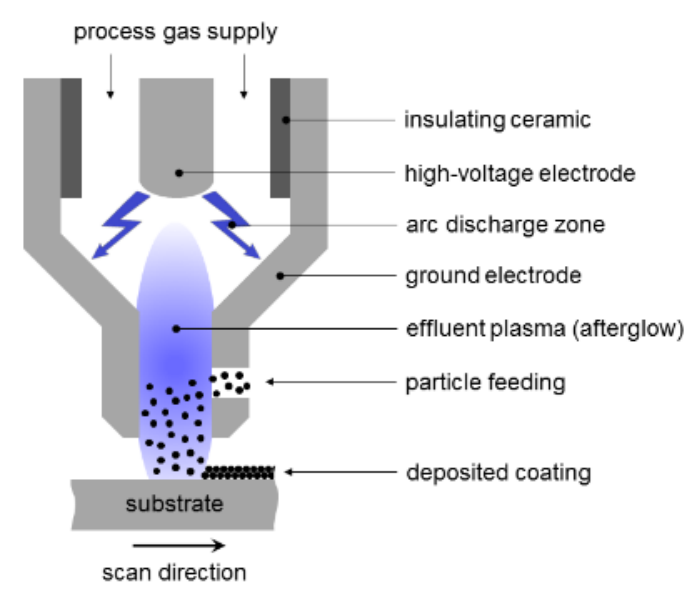

Figure 3: Assembly to deposit particle coatings by cold plasma spraying (Paper I).

To generate the particle aerosol, the powder disperser RBG 2000 from Palas was applied. Here, the dry powder is uniformly compressed to yield a powder column. This powder column is moved towards a rotating brush at a constant velocity which defines the feed rate. Above the brush, the carrier gas takes up the particles and transports them towards the discharge. Below the spraying nozzle, the sample is mounted on a xy-linear stage to obtain a coating of the entire surface.

Basically, particles are assumed to be melted in the plasma and blown on the substrate where they solidify and therefore adhere through micromechanical clamping. Since the temperatures in cold plasma spraying remain comparatively low, limitations caused by their melting points exist for applicable powder materials. Moreover, adhesion by the formation of chemical bonds or electroadhesion are conceivable mechanisms for some types of powder material.

The coating process was adjusted by a variety of parameters. Each combination of powder and substrate material together with the choice of a specific process gas needed a specific tuning of the parameters to achieve homogeneous coatings and concurrently inhibit thermal damage to the sample. Commonly varied parameters included:

- plasma power,

- volume flow rate of process gas,

- pressure of carrier gas,

- working distance between spraying nozzle and substrate,

- substrate displacement velocity,

- powder feed rate. 
Furthermore, the particles' size and shape was found to have a crucial impact on the quality of the coating, particularly with regard to homogeneity (cf. Chapter 4).

The setup for cold plasma spraying as described above represents a promising approach to applying protective coatings on thermosensitive wooden surfaces. Various powder materials like metals or polymers are suitable coating materials, therefore ensuring flexibility with regard to the design of surface properties. Particularly because of the absence of vacuum setups, the technique is suitable for the in-line processing of large-scale objects. Even on a laboratory scale, coating velocities of up to $150 \mathrm{~mm} / \mathrm{s}$ are possible. Due to the application of a jet, the assembly could easily be modified to coat different sample geometries by mounting it on a robotic arm. Moreover, solvents or binding agents are not included in this deposition process, thus preventing probably dangerous emissions - for example, of volatile organic compounds (VOCs).

On the other hand, cold plasma spraying faces some challenges. For industrial applications, a reliable encapsulation of the coating area is mandatory in order to avoid occupational risks. Furthermore, the adhesion and abrasion resistance of the particle coatings needs to be investigated carefully. Particle coatings on wood in general can emit nanoparticles - for example, due to abrasion by windblown sand particles [147]. The general risk assessment of nanoparticles is still ongoing work, but many studies have found dangers for human health and the environment from several types of nanoparticles [148,149].

\subsection{Surface analytical methods}

This section briefly presents the analytical methods predominantly applied to characterise deposited particle coatings. The measurement principles are summarised with a focus on the aspects necessary for the applications throughout this work.

\subsubsection{Methods to investigate morphologic properties}

The resulting morphology of different particle coatings was mainly studied by Laser Scanning Microscopy (LSM), Atomic Force Microscopy (AFM), and Scanning Electron Microscopy (SEM). The combination of these techniques made it possible to visualise diverse aspects of the structures on different scales, ranging from the nanometre scale to micrometric dimensions.

For LSM measurements [150], a laser beam is scanned across the sample's surface. A confocal pinhole ensures the detection of light reflected only in the focal plane. By a welldefined displacement of the sample in z-direction, it is scanned layer by layer, eventually 
yielding a height map of the surface. Different magnifications and thus frame sizes were used, but in most cases, a scanned area of $320 \times 320 \mu \mathrm{m}^{2}$ was found to be suitable for the purpose of these studies. This technique operates under ambient conditions and does not require any further sample preparation. Therefore, it permits quick and non-destructive 3D-imaging even of rather thick samples. The shape and size of individual particles or agglomerates as well as surface roughness parameters can be assessed. However, scattering of light, particularly by metallic particles, can lead to distortion.

To obtain height information on a scale beyond optical resolution limits, AFM measurements were carried out. During AFM measurements, a flat and very thin probe (diameter of some $\mathrm{nm}$ ) attached to a spring scans the sample's surface. Height information is extracted from the deflection of the spring resulting from atomic forces between the surface and the probe with a resolution below the nanometre scale [151]. On particle-coated glass surfaces, a frame size of $3 \times 3 \mu \mathrm{m}^{2}$ was found to yield the most informative data on the roughness of the coatings as well as on the shape and size of individual particles on a much smaller scale compared to LSM measurements. AFM measurements can be carried out under ambient conditions and therefore require little sample preparation. However, the application on rough surfaces such as wood may pose a challenge due to a limited vertical lift.

Moreover, SEM measurements were performed. During SEM measurements, a focused electron beam (primary electrons, PE) is scanned across the sample's surface and thus produces various signals through interactions between the electron beam and the surface atoms [152]. In addition to a signal created from backscattered electrons, secondary electrons (SE) may be detected. These are electrons from the valence band of the surface atoms being released by inelastic scattering with the primary electrons. Since the intensity of the detected SE signal strongly depends on the local inclination of the surface with respect to the primary electron beam (i.e. the interaction volume), this signal can be used to visualise the morphology. SEM stands out due to its high lateral resolution and large depth of focus. Throughout this work, areas sized $48 \times 64 \mu \mathrm{m}^{2}$ were commonly investigated. However, the measurements usually need to be carried out under high vacuum and thus, only very small samples can be analysed. Moreover, insulating samples must usually be coated by a thin conductive layer to avoid charging.

\subsubsection{Methods to investigate chemical properties}

Chemical issues were mainly investigated by X-Ray Photoelectron Spectroscopy (XPS) and Fourier Transform Infrared Analysis (FTIR). 
XPS permits surface-sensitive measurements of the chemical composition with an information depth of up to approximately $10 \mathrm{~nm}$. For XPS measurements [153], X-rays irradiate the sample's surface and cause an electron release by the photoelectric effect. Since the energy of the incident light is well-defined, the binding energy of the photoelectrons may be calculated from their measured kinetic energy ${ }^{18}$, leading to peaks at binding energies characteristic for the element as well as for the chemical bonds involved. In addition to these photoelectrons, Auger electrons are generated due to the relaxation of excited ions, where an electron from an outer energy level falls into an inner vacancy, causing the emission of a second electron to ensure energy conservation. These additional characteristic peaks in the XPS spectra may support species identification. XPS measurements were one core topic throughout this work. For quantitative analysis, a Shirley-type background [154] was first subtracted to remove contributions from photoelectrons which undergo inelastic scattering before leaving the surface and thus yield an apparent binding energy exceeding those of the corresponding element's characteristic photoemission peak. Since the binding energy of these peaks depends not only on the particular element but also on the chemical bonds involved (molecular orbit energies) $[155,156]$, the resulting chemical shift leads to photoemission peaks which usually are the sums of several subpeaks. The chemical bonds of a specific element can therefore be determined by spectral deconvolution of the corresponding total peak. In this way, for example, an oxidation or depolymerisation of PMMA was shown to result from different plasma treatments (cf. Chapter 5). The line shape of the (sub-)peaks is usually represented by a convolution of a Gaussian and a Lorentzian function ${ }^{19}$, since the profile is determined by both the natural broadening (core-hole lifetime, Lorentzian) and instrumental influences (e.g. resolution of the analyser, typically Gaussian) [155]. Final-state effects can further affect the peak profile; for example, metals usually feature an asymmetric peak shape since the escaping photoelectron can excite valence band electrons which are transferred into the conduction band. For this process, a continuous range of energies is available (conductor) and by losing some of their kinetic energy, the photoelectron's calculated binding energy appears slightly higher. Such asymmetric peak shape was indeed observed for elemental zinc in this work (cf. Chapter 4.1). Because the zinc peaks of

18 The binding energy $E_{B}$ is calculated from the kinetic energy $E_{k i n}$ and the photon energy $h v$ through: $E_{B}=h v-E_{k i n}-\Phi_{\text {spec }}-E_{c h}$, with corrections for the spectrometer workfunction $\Phi_{\text {spec }}$ and charging effects $E_{c h}$.

19 For practical reasons, the convolution is approximated by a linear combination of the two contributing functions. 
elemental zinc and zinc oxide suffer from a strong overlap that complicates thorough deconvolution, an Auger peak was also studied to support species identification qualitatively. The particular $\mathrm{L}_{3} \mathrm{M}_{45} \mathrm{M}_{45}$ zinc Auger peak was chosen because it is clearly visible in the spectrum with distinctly separated binding energies for elemental zinc and zinc oxide. Moreover, it is generated by the electrons contributing to the photoemission peak that was mainly studied (energy level $\mathrm{L}_{3}$ or $\mathrm{Zn} 2 \mathrm{p}_{3 / 2}$ ). In addition, depth profiles were assessed by alternating acquisition of spectra and etching with argon ions in order to study the oxidative and elemental moieties below the uppermost surface.

In general, XPS measurements are surface sensitive, capable of detecting every element except hydrogen and helium, and permit the determination of the elemental composition independent of the chemical states. However, measurements need to be carried out under ultra-high vacuum, which leads to challenges when performing measurements on outgassing samples such as wood. Moreover, even though the identification of some functional groups is generally possible (valence band region), the required analysis is comparatively complex and thus, sole XPS measurements are usually not used for this purpose.

A method well-suited for the identification of functional groups is FTIR analysis. This technique measures the characteristic absorption peaks resulting from the molecular vibrations induced by interaction with light, usually in the mid-infrared region between $4000 \mathrm{~cm}^{-1}$ and $400 \mathrm{~cm}^{-1}[157,158]$. The characteristic spectra permit a direct identification of functional groups. Common acquisition techniques include the assessment of spectra via transmission of light which requires only small amounts of the substance of interest (usually $1 \mu \mathrm{g}$ ). For opaque wood samples, spectra may instead be recorded by the use of Attenuated Total Reflectance (ATR-FTIR). Here, the sample is pressed onto a crystal in which the infrared light is guided in total internal reflectance. Absorption characteristics are generated through interactions of the resulting evanescent waves at the interface between the crystal and the sample with the sample's surface molecules. Since the penetration depth of these evanescent waves depends on the specific wavenumbers, the relative ATR intensities must be corrected prior to comparison with the transmittance spectra. ATR-FTIR copes without further sample preparation and permits non-destructive measurements. It is therefore wellsuited, for example, to studying chemical changes on wood surfaces induced by UV light, which requires that the sample's original state be maintained after the initial chemical analysis. The direct detection of functional groups enables, for example, a distinction between lignin and cellulose/hemicellulose. Valuable information on some samples was also able to be extracted from difference spectra. The subtraction of spectra acquired, for example, after and 
before exposure to UV light can highlight changes; however, it requires preceding data processing that might not be possible for specific samples. Following thorough baseline correction, this method demands normalisation to a reference peak which is unaffected by the investigated treatment. If all chemical moieties change, specifying a reference peak is not possible.

(ATR-)FTIR measurements are therefore well-suited for determining functional groups and can even permit non-destructive measurements. On the other hand, however, the information depth is significantly higher (up to several $\mu \mathrm{m}$ ) than for XPS; therefore, bulk properties influence the results. Moreover, the elemental composition cannot be determined independent of the chemical bonds involved. 


\section{Deposition of Zinc/Zinc Oxide Particles}

This chapter summarises studies on coatings composed of zinc and zinc oxide. First, the fundamental properties of the coatings were investigated on homogeneous substrates to avoid bias from a complex substrate such as wood. Subsequently, studies on the applicability of zinc/zinc oxide coatings to reduce fungal infestation or photodegradation are presented.

\subsection{Characterisation of layers deposited on glass substrates}

To estimate if the coatings applied by cold plasma spraying with elemental zinc as the base material are suitable candidates for further applied studies on their protective potential, some fundamental properties of the particle layers were investigated on coated homogeneous glass substrates (Paper I). The focus was on studies of topographic, optical, and chemical properties.

The applied coatings were adjusted to yield almost transparent layers on the surface ${ }^{\mathbf{2 0}}$ in order to minimise changes to its appearance. A powder containing flake-like zinc particles with a diameter of $\mathrm{d}_{50}=13 \mu \mathrm{m}$ served as the base material. As ascertained by LSM and AFM measurements, the coatings consisted of sporadically distributed larger particles and accumulations with sizes in the $\mu \mathrm{m}$-range, both smaller and slightly larger than the initial diameter. In between such accumulations, an almost closed layer of particles in the nm-range was generated, probably resulting from the evaporation of zinc in the plasma and condensation on the sample's surface.

One targeted application of such coatings is the protection of wood surfaces from photodegradation. Therefore, transmittance spectra of coated UV-transparent acrylic glass were acquired. A representative spectrum is depicted in Figure 4, which shows a strong absorption edge in the UV range. From such spectra, a band gap energy of $(3.2 \pm 0.02) \mathrm{eV}$ was calculated. Hence, considerable UV-blocking properties of the coatings were confirmed. 


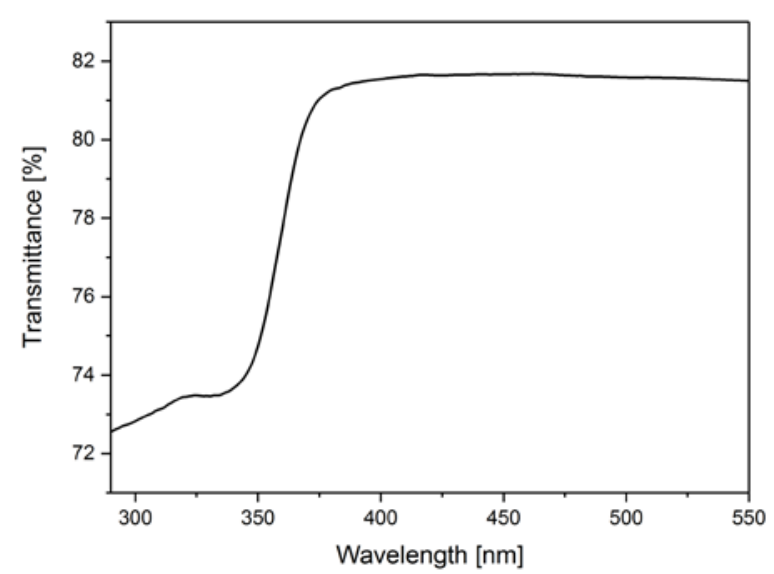

Figure 4: Absorption edge in the UV range of the transmittance spectrum of coated acrylic glass (Paper I).

The coatings are supposed to contain zinc oxide; however, elemental zinc was used as the base material. Therefore, studies on the chemical composition were carried out to investigate the formation of zinc oxide. XPS measurements on coated fused silica confirmed the presence of a significant share of oxidised zinc also below the uppermost surface, as ascertained by depth profiling. Moreover, the nanostructures observed in the AFM measurements were affirmed to be composed of zinc compounds.

In summary, studies on the topographic, optical, and chemical properties of almost transparent coatings applied by cold plasma spraying with elemental zinc as the base material confirmed the suitability of such coatings as protective layers. Chemical analysis affirmed the formation of zinc oxide, and optical measurements revealed significant UV-blocking properties. As ascertained by topographic measurements, an almost closed layer of nanostructures is located between accumulations of larger particles.

\subsection{Exposure of zinc/zinc oxide-coated wood to blue stain}

To study the antimicrobial effect of coatings partly consisting of zinc oxide, their impact on the growth of blue stain under laboratory conditions was studied (unpublished results). Even though these tests did not show any difference between the fungal infestation of coated and uncoated samples, the results could be used to optimise the particle coatings. The failure of protection is assumed to be mainly due to an insufficient surface density/concentration ${ }^{21}$ of particles on the surface. Moreover,

21 A decrease in the particle size (use of nanoparticles) and thus an increase in specific (reactive) surface area could lead to similar effects as an increased concentration of microparticles. However, particle fixation needs to be ensured as well. 
leaching of the particle coatings was observed, leading to a further reduction of the particle concentration.

For blue stain tests, wood specimens from Scots pine (Pinus sylvestris L.) with a size of $45 \times 40 \times 10 \mathrm{~mm}^{3}$ were used. Coatings were realised by applying a process gas (air) flow rate of $30 \mathrm{~L} / \mathrm{min}$, a carrier gas (air) pressure of $0.16 \mathrm{MPa}$, a distance of $23 \mathrm{~mm}$ between the spraying nozzle and the substrate, and a substrate displacement velocity of $50 \mathrm{~mm} / \mathrm{s}$. To achieve two different concentrations, the particle feed rate was set to $3.2 \mathrm{~cm}^{3} / \mathrm{h}$ (coating A) and $6 \mathrm{~cm}^{3} / \mathrm{h}$ (coating B). The test was carried out basically according to the standard DIN EN 152 [159] by applying the fungus Aureobasidium pullulans (de Bary) Arnaud. In this way, six replicates per treatment (coating A, coating B, control) were sterilised by exposure to gamma radiation at Synergy Health Ede BV (Schiedam, The Netherlands). Inoculation was attained by dipping the coated surface into spore suspension and subsequently placing the sample's uncoated rear side onto vermiculite which also contained spore suspension. In addition, a second set of samples was prepared. Analogous to [28], the coated surface was placed directly onto the inoculated vermiculite. To prevent an unintentional spreading of the fungus beyond the coated surface, all lateral faces were sealed. Incubation lasted for six weeks at $22{ }^{\circ} \mathrm{C}$ and $70 \%$ relative humidity. Fungal infestation was assessed by visual inspection and classification from 0 (no blue staining) to 3 (strong blue staining), and by measuring the length of the zone without penetration of the fungal hyphae after cutting the specimens. Figure 5 depicts representative images of surfaces after direct contact with the vermiculite containing fungal spores (i.e. particle-coated surface in direct contact) showing no significant difference between the uncoated and coated specimen.

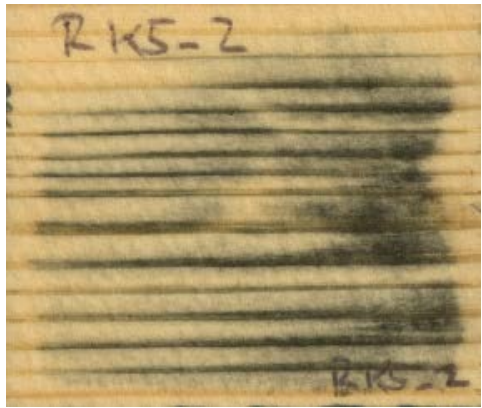

(a)

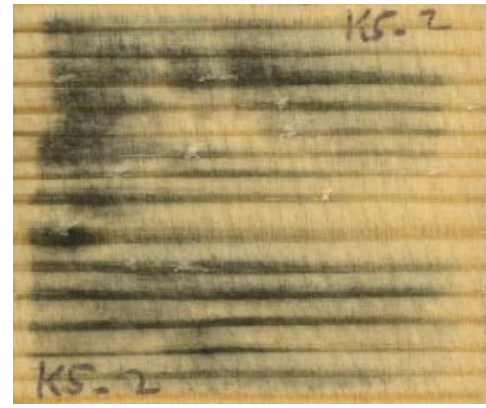

(b)

Figure 5: Blue staining of Scots pine after six weeks of incubation. Left: uncoated specimen. Right: particle-coated sample after direct contact with the inoculated vermiculite.

Table 1 (coated surfaces in contact with the vermiculite) and Table 2 (coated surface opposite to the vermiculite) summarise the results assessed according to [159]. In consideration of the 
inaccuracies inherent to the classification methods, no significant differences between the fungal infestation of coated and uncoated wood specimens were found.

Table 1: Classification of the surfaces from rank 0 (no blue staining) to 3 (strong blue staining) along with the length of the area free from fungal infestation according to [159]. The particle-coated surface was in direct contact with the inoculated vermiculite.

\begin{tabular}{lccc}
\hline Samples & Contact side & Rear side & $\begin{array}{c}\text { Depth without } \\
\text { infestation in } \mathbf{~ m m}\end{array}$ \\
\hline Control & $3 \pm 0$ & 0 & $6.3 \pm 1.7$ \\
Coating A & $2.8 \pm 0.4$ & 0 & $7.7 \pm 1.9$ \\
Coating B & $2.8 \pm 0.4$ & 0 & $7.6 \pm 1.6$ \\
\hline
\end{tabular}

Table 2: Classification of the surfaces from rank 0 (no blue staining) to 3 (strong blue staining) along with the length of the area free from fungal infestation according to [159]. The particle-coated surface was on the surface opposite to the inoculated vermiculite.

\begin{tabular}{lccc}
\hline Samples & Contact side & Rear side & $\begin{array}{c}\text { Depth without } \\
\text { infestation in } \mathbf{~ m m}\end{array}$ \\
\hline Control & $2.8 \pm 0.4$ & $1.8 \pm 1$ & $7.4 \pm 2.1$ \\
Coating A & $3 \pm 0$ & $1.5 \pm 0.8$ & $6.3 \pm 2.4$ \\
Coating B & $3 \pm 0$ & $1.3 \pm 1$ & $6.6 \pm 1.8$ \\
\hline
\end{tabular}

Up to this point, the coatings were designed to yield (almost) transparent layers to keep the wood's natural appearance. Even though measurements on homogenous substrates confirmed the presence of zinc oxide, actual applications seemed to demand higher concentrations. In addition, these studies on the growth of blue stain were the first tests that required a coating of larger substrates and revealed problems in the homogeneity of large-scale coatings. Based on these results, several different zinc base materials were tested. A powder consisting of particles featuring an irregular shape and a diameter of $\mathrm{d}_{50}=16 \mu \mathrm{m}$ was found to increase the quality of the coatings significantly compared to the flake-like particles with a diameter of $\mathrm{d}_{50}=13 \mu \mathrm{m}$ which were used in this and previous (Paper I) studies.

Hence, preliminary tests investigating the impact of zinc/zinc oxide particle coatings on the infestation by blue stain did not show any difference between coated and uncoated wood specimens. The major reason for the failure is assumed to be an insufficient particle concentration. During the execution of these studies, leaching of the particle coatings was observed, revealing the need for a proper particle fixation. Moreover, the challenge of ensuring homogeneity in large-scale coating became apparent. In the following studies on the 
photoprotection of wood, coating systems comprised of a particle coating and additional conventional sealing were investigated to find suitable materials that would ensure particle fixation. In addition, zinc particles featuring an irregular shape were used as the base material to improve the homogeneity of the coatings.

\subsection{Exposure of zinc/zinc oxide-coated wood to UV light}

This section presents studies on the particle coatings' impact on the photodegradation of wood and potential sealing materials in artificial ageing tests. Moreover, morphologic properties of the optimised zinc/zinc oxide coatings on wood were investigated (Paper II).

Even though thin, transparent coatings led to an increased absorption of UV light (cf. Figure 4 and Paper I), the effect was not considered to reduce photodegradation sufficiently. Therefore, the particle concentration was increased, leading to a greying of the surface. Moreover, coating systems containing a particle coating and a conventional sealing were studied. In preliminary tests, particle-coated samples were sealed with an alkyd paint (ProfiDur Klarlack, Schöner Wohnen Farbe), polyurethane (SKresin P34 NV, S u. K. Hock), epoxy resin (SKresin V6, S u. K. Hock) or an acrylic binder (Acronal LR 9014, BASF) and exposed to UV light. The acrylic sealing appeared to degrade very rapidly, presumably through photocatalytic decomposition, and was therefore rejected. All other sealings seemed to be adequately stable; therefore, the alkyd and the PUR sealing were chosen for further studies.

The coatings' morphology was examined by scanning electron microscopy (SEM) and roughness measurements. Two different concentrations were realised: coating A represented a rather thin coating, and coating B was an example of a thicker coating. SEM measurements on coated beech samples confirmed a melting and solidifying process as was expected to occur during cold plasma spraying, leading to the formation of coatings composed of very flat particles that did not completely screen the wood structure for either coating. Roughness measurements ${ }^{22}$ on coating B suggested that the particles were even incorporated in the wood structure to some extent (e. g. in wood pores).

Unsealed and PUR- or alkyd-sealed samples were exposed to UV light for a total of 50 hours. Changes induced by artificial ageing were studied through colour measurements and ATRFTIR. For the colour measurements, pictures were taken under equal conditions. CIE Lab 
parameters were calculated by using the software Photoshop CS6. This system describes colour values by the parameters $L, a$, and $b$, with $L$ being the lightness in grey-scale values, $a$ representing the red-green colour axis, and $b$ corresponding to the blue-yellow axis ${ }^{23}$. The total colour change $\Delta E$ is defined as:

$$
\Delta E=\sqrt{\left(L_{0}-L_{i}\right)^{2}+\left(a_{0}-a_{i}\right)^{2}+\left(b_{0}-b_{i}\right)^{2}} .
$$

Figure 6 shows the variation of $\Delta E$ with increasing irradiation time for unsealed samples. Compared to uncoated samples, the thin coating (coating A) was significantly able to reduce colour changes induced by UV irradiation. Colour changes of samples coated with a thick layer of zinc/zinc oxide particles (coating B) remained almost negligible. Similar improvements were observed for particle-coated and sealed samples.

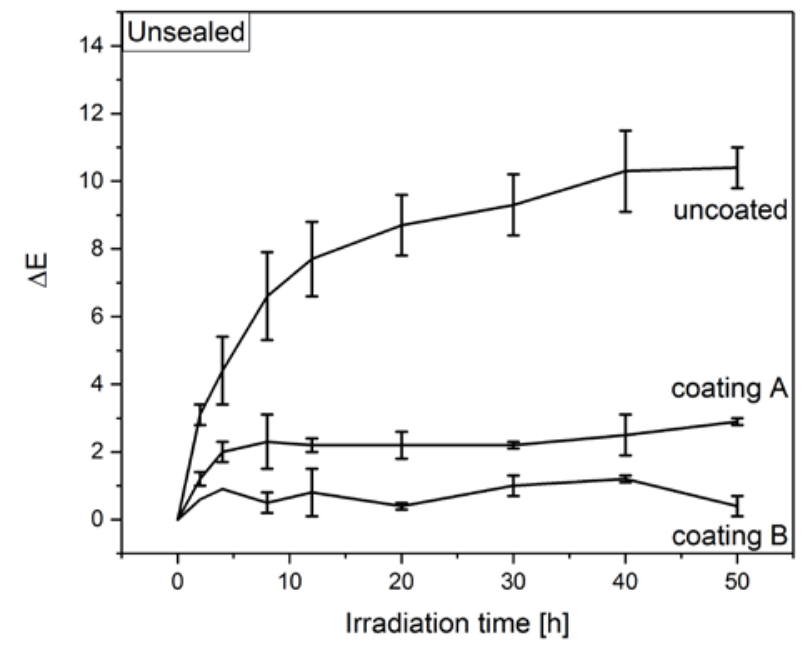

Figure 6: Variation of total colour change with irradiation time for the unsealed samples (Paper II).

To assess chemical changes induced by UV irradiation, ATR-FTIR measurements were carried out. Uncoated and unsealed reference samples showed, as expected, a significant reduction of lignin on the surface. PUR-sealed samples underwent only slight changes upon UV irradiation in the absence of zinc/zinc oxide particles and even seemed to be slightly stabilised by adding a particle coating. On the other hand, alkyd-sealed samples exhibited distinct chemical changes. These effects strongly increased in the presence of a particle coating, thus indicating photocatalytic decomposition processes.

23 $\Delta a=a_{0}-a_{i}>0$ denotes a green shift for the $i$-th measurement, $\Delta a<0$ a red shift, $\Delta b=b_{0}-b_{i}>0$ a blue shift, and $\Delta b<0$ a yellow shift. 
In summary, the deposited particle coatings were significantly able to reduce colour changes induced by photodegradation. The PUR sealing underwent only negligible chemical changes during artificial ageing and seemed to be stabilised by a particle coating. On the other hand, alkyd-sealed samples exhibited strong chemical changes, particularly for coating systems involving a particle layer. Therefore, a coating system consisting of a particle coating and the PUR sealing was identified as a promising candidate for further studies.

\subsection{Discussion}

The results presented in Chapter 4.1, 4.2 and 4.3 showed the feasibility of cold plasma spraying for the deposition of protective $\mathrm{ZnO}$-containing layers on wood and other substrates. The characterisation of thin coatings on glass substrates showed the generation of mixed micro- and nano-scaled zinc and zinc oxide structures that successfully reduced the transmittance of ultraviolet light. An exposure to blue stain did not show any increased resistance induced by such a thin coating; therefore, the particle concentration was increased for subsequent UV tests and sealings were studied which should enhance particle fixation. With these optimised coatings, the photostability of beech wood could be significantly enhanced.

Throughout this work, the coatings were prepared from particles featuring an initial diameter in the micrometre range, leading to the generation of both micro- and nano-scale particles on the surface. In contrast, several other research groups have successfully applied zinc oxide nanoparticles to reduce photodegradation of wood[160-164]. Among other things, nanoparticles feature a particularly high specific surface area and thus an increased reactivity compared to the bulk material. Indeed, these studies were able to decrease discolouration of wood by UV irradiation despite an impressively low concentration of $\mathrm{ZnO}$ (suspensions with up to 4 wt. \% of $\mathrm{ZnO}$ ) and thus a semitransparency of the layers. However, even though photodiscolouration could be reduced, distinct colour changes were still measured. For this reason, Miklečić et al.[163] proposed the addition of pigments which would, however, prevent semitransparency. In comparison, the greyish coatings used in this work were (almost) completely able to prevent photodiscolouration; and the use of micro- instead of nanoparticles should decrease occupational hazards. Nevertheless, the use of nanoparticles presents a promising approach and could be explored in future studies. To avoid severe agglomeration of a powder, approaches to synthesising nanoparticles directly in the plasma could be explored $[165,166]$. 
During this work, a reliable quantification of the particle concentration was not possible. Standard methods such as $\mathrm{ICP}^{24}$ - atomic emission spectroscopy were not available, and simple methods such as weighing the sample before and after coating were not considered to be adequate due to the possible further effects of the plasma treatment on wood surfaces such as etching and drying. Therefore, an exploration of methods to assess the particle load thoroughly would be crucial for ongoing studies related to the influence of the deposition parameters on the resulting particle concentration, and to improve comparability with results from other research groups. Moreover, leaching of the coatings and thus the efficiency of the sealing was not able to be investigated systematically yet.

In an attempt to achieve both the sealing of particle coatings and water repellence in one process step, two monomers were tested with regard to their potential to become polymerised in the effluent plasma of the setup for cold plasma spraying. Even though the trials were carried out without the addition of particles, a concurrent injection of particles and monomers would be possible. On the one hand, octafluorocyclobutane $\left(\mathrm{C}_{4} \mathrm{~F}_{8}\right)$ was added to the plasma, because it can be used to create hydrophobic layers by plasma polymerisation [167-169]. However, no significant increase in water contact angles was measured and therefore, this approach was not pursued further. On the other hand, HMDSO was injected into the plasma. The characterisation of the surfaces indeed showed a layer formation which, depending on the deposition parameters, was able to exhibit a highly hydrophobic nature. However, the coatings could easily be wiped off and were therefore not eligible to ensure particle fixation. LSM and AFM measurements suggested the formation of small particles in the plasma which are subsequently blasted onto the surface. An injection of the monomer much closer to the substrate might permit film growth on or close to the surface, but a further reduction of the distance could not be achieved without elaborate changes to the deposition apparatus. Nevertheless, this approach could be pursuit further in future studies.

Given the fact that coatings including $\mathrm{ZnO}$ nanoparticles can also be applied by dispersing the particles in a conventional varnish [160-164], the sequential application of particles by cold plasma spraying and additional sealing might appear inconvenient. However, this procedure features advantages since an optimisation of the method might permit a precise dosage of the UV-filters and the top coat. Due to the absence of particles in the sealing, an unintentional release of (nano-)particles by leaching or abrasion by windblown particles might be inhibited. 
Moreover, as discussed in the literature [161,163], the preparation of a suspension that can be applied like conventional varnishes is non-trivial and may be time consuming since an agglomeration of the particles has to be avoided and the suspension is usually not stabilised. Pre-dispersed suspensions may contain stabilisers that decrease efficiency by shielding the $\mathrm{ZnO}$ nanoparticles [160]. Therefore, an optimisation of the procedure to deposit $\mathrm{ZnO}$ containing coatings applied throughout this work appears worthwhile. 


\section{Deposition of Poly(methyl methacrylate)/Aluminium Trihydrate Particles}

This chapter summarises studies on coatings with PMMA/ATH as the base material. Initially, pure PMMA/ATH powder was deposited without additives, leading to coatings with insufficient abrasion resistance. The abrasion resistance could subsequently be strongly enhanced by the addition of a phenol-formaldehyde powder.

\subsection{Characterisation of pure poly(methyl methacrylate)/aluminium trihydrate coatings on wood}

Pure PMMA/ATH coatings on wood were investigated with regard to their topographic and chemical properties as well as wettability (Paper III). Based on the results, changes induced by the plasma treatment could be revealed and the potential to improve bonding between the powder and possible matrix materials in order to enhance particle fixation was evaluated.

Particle coatings were obtained by the deposition of PMMA/ATH powder using compressed air as the process and carrier gas. To avoid damage to the coating facility, the powder was sieved previously to contain only particles featuring a diameter of less than $63 \mu \mathrm{m}$. Three different concentrations were realised by adjusting the distance between the spraying nozzle, the powder feed rate, and the carrier gas pressure.

The general chemical structure of PMMA is shown in Figure 7 and the results from the chemical analysis are summarised in Table 3. A strong increase of aluminium on the surface, together with the decrease of carbon, indicated etching of the PMMA matrix and thus exposure of the filler particles. Moreover, oxidation of the PMMA occurred to some extent, leading to a relative increase in $\mathrm{O}-\mathrm{C}=\mathrm{O}$ bonds. These chemical changes may be favourable for an improved bondability.

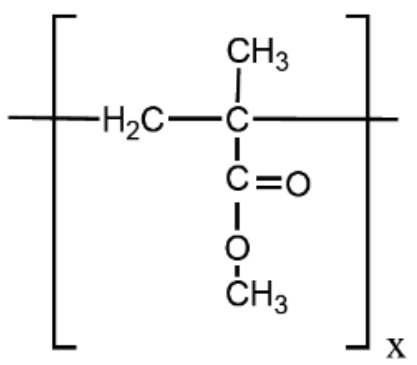

Figure 7: Che mical structure of PMMA [170]. $x$ refers to the number of building units. 
As ascertained by $\mathrm{LSM}^{25}$, the deposition process did not lead to film formation even though the powder was exposed to temperatures exceeding the composite's glass transition temperature. Thus, a very rough structure composed of individual particles was obtained. Contact angle measurements revealed a very high wettability by water and a disperse liquid (diiodomethane), thus suggesting the suitability of both polar and apolar liquids for embedding the particles in a matrix and ensuring fixation. The high wettability may result from the rough structure on the one hand, and the chemical changes on the other hand. Particularly the exposure of ATH should promote adhesion to a polar top-coat matrix because it is more likely to form chemical bonds than PMMA - for example, through hydrogen bonds.

Table 3: Chemical changes induced by the plasma treatment (data adapted from Paper III). The concentrations of the particular elements or chemical bonds are summarised together with the measured relative area $A_{\text {rel }}$ of the carbon bonds within the C1s peak to highlight changes in the organic structure.

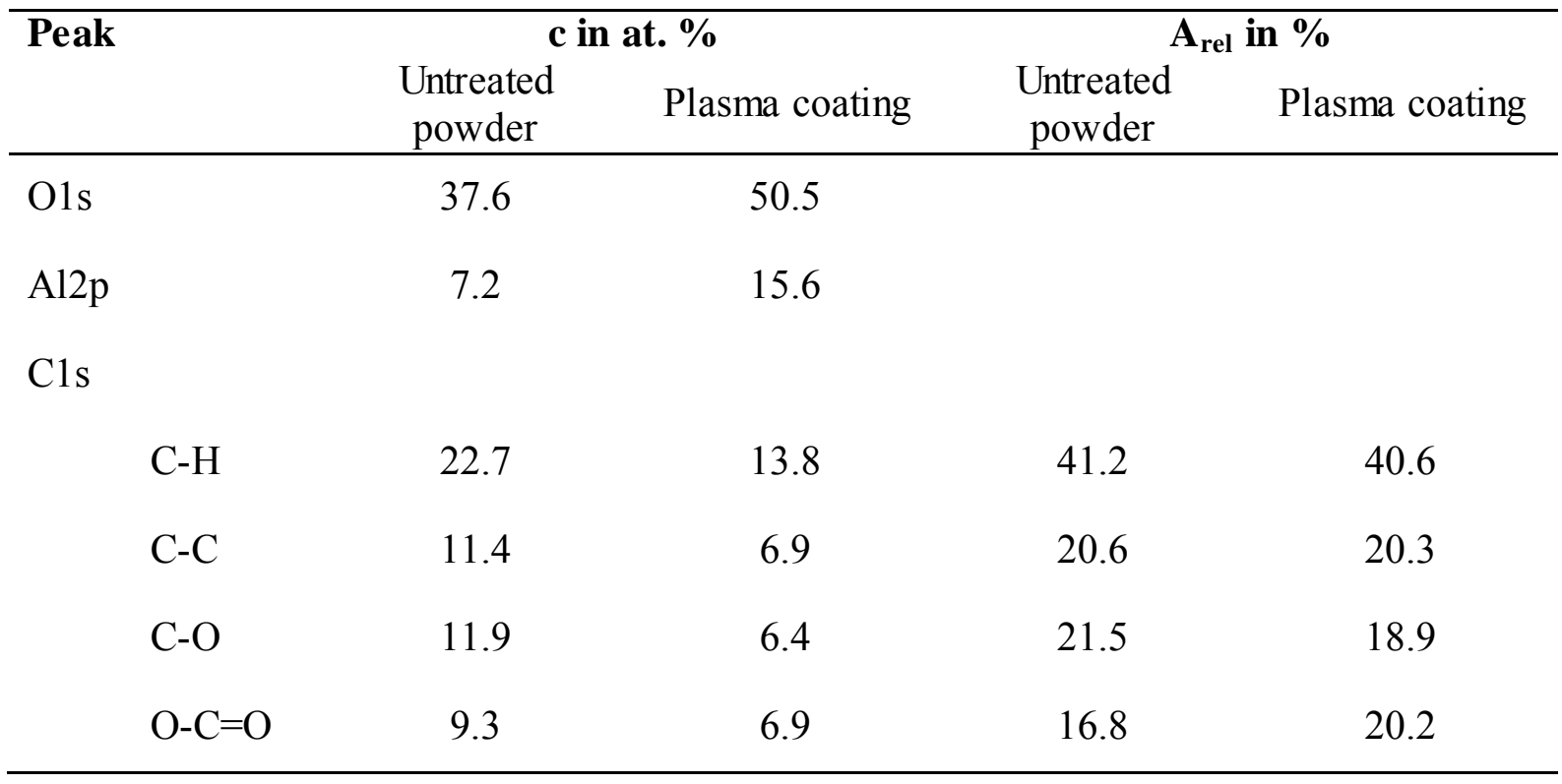

In summary, the characterisation of PMMA/ATH layers on wood confirmed the material's suitability as a coating material deposited by cold plasma spraying. Despite an insufficient abrasion resistance, the chemical changes induced by the plasma treatment together with the high roughness and wettability by polar and apolar liquids suggest the eligibility for an embedding in a matrix material to ensure particle fixation. Therefore, the approach appears suitable for developing an adequate recycling option for PMMA/ATH waste powder. gratefully acknowledged. 


\subsection{Enhanced abrasion resistance of poly(methyl methacrylate)/aluminium trihydrate coatings on wood and glass substrates}

In an attempt to stabilise PMMA/ATH coatings, two different modifications of the basic deposition process were studied. Abrasion tests were carried out to evaluate the efficacy of the two approaches (Paper IV). To elucidate the processes occurring during the plasma treatment, the different coatings on wood and glass were characterised with a focus on their morphologic and chemical properties as well as wettability. Moreover, the coatings' adhesion was studied to complement the abrasion tests (Paper V).

In the first approach to creating wood coatings based on PMMA/ATH powder, compressed air was used as process and carrier gas (hereafter referred to as coating A). Compressed air was found to be feasible for the deposition of various powder materials and it contributes to keeping process costs relatively low. In a first trial, this process and carrier gas was replaced by forming gas to test a reducing environment (coating B). XPS measurements and particularly the investigation of the carbonic structure confirmed severe depolymerisation of PMMA in the plasma, presumably by depletion of methyl/methylene groups and chain scission. Abrasion and adhesion tests showed, however, that these modifications did not lead to improved particle fixation. Accordingly, SEM measurements did not show any evidence for film formation.

In a second approach, the PMMA/ATH base material was mixed with a powdered novolac phenol-formaldehyde ( $\mathrm{PF}$, yielding samples with coating $\mathrm{C}$ ). The general formula of a novolac PF is depicted in Figure 8.

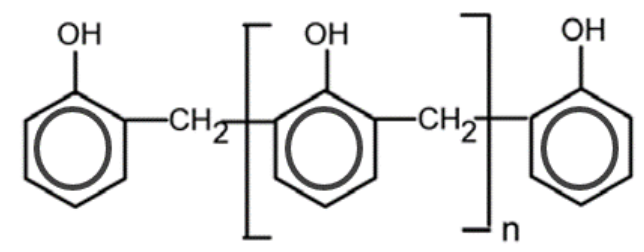

Figure 8: Chemical structure of a novolac phenol-formaldehade resin (adapted from [171]).

Abrasion and adhesion tests revealed an outstanding bonding between the coating and wood and particularly between the coating and glass substrates. The bonding between individual particles/agglomerates within the coating was found to be improved but not sufficient for applications yet. As ascertained by SEM, the particles seemed to fuse, presumably by an envelope of reacted PF around individual PMMA/ATH particles. On the other hand, piling of particles on the substrates was observed, thus leading to large spatial distances between the 
particles/agglomerates within the coatings. The failure to ensure sufficient bonding within the coatings is therefore assumed to result from the coating's morphology. Chemical analysis by both FTIR and XPS showed a strong dominance of reacted PF in the coating. The elemental composition as assessed by XPS is shown in Table 4. Compared to the untreated powder, coating $\mathrm{C}$ featured notably less aluminium and oxygen on its surface. Concurrently, the nitrogen and carbon content increased. Since the untreated PF powder comprised significantly more carbon than PMMA and additionally included a nitrogen compound (hexamethylenetetramine, HMTA), these findings supported the hypothesis that reacted PF encased the PMMA/ATH particles. Moreover, investigation of the carbonic structure showed an increase in oxidative compounds. As an adhesion mechanism of PF on wood, Laborie and Frazier [172] proposed interactions of the PF's hydroxyl groups with the different wood constituents. Similarly, the outstanding adhesion of the coating on glass may result from interactions between the resin's hydroxyl groups and $\mathrm{Si}-\mathrm{OH}$ bonds on the glass surface in the presence of an activating plasma, i.e. formation of Si-O-C bonds and release of water. Moreover, water contact angle measurements revealed the hydrophobic nature of the coating, with angles constantly above $100^{\circ}$.

Table 4: Elemental composition of the different samples as determined by XPS together with the resulting ratio O/C (data adapted from Paper V). Mean values of three measurements are displayed together with standard deviations in parenthesis. Contributions from Na contamination are not listed.

\begin{tabular}{lccccc}
\hline Sample & $\begin{array}{c}\text { O1s } \\
\text { c in at. \% }\end{array}$ & $\begin{array}{c}\text { C1s } \\
\text { c in at. \% }\end{array}$ & $\begin{array}{c}\text { Al2p } \\
\text { c in at. \% }\end{array}$ & $\begin{array}{c}\text { N1s } \\
\text { c in at. \% }\end{array}$ & O/C \\
\hline Untreated PMMA/ATH & 49.8 & 38.4 & 11.8 & & 1.3 \\
& $(0.8)$ & $(0.5)$ & $(0.4)$ & & \\
Coating A & 50.9 & 32.1 & 15.6 & & 1.6 \\
(PMMA/ATH in air) & $(0.2)$ & $(0.4)$ & $(0.3)$ & & 1.3 \\
Coating B & 47.8 & 37.5 & 13.7 & & \\
(PMMA/ATH in $\left.\mathrm{H}_{2}+\mathrm{N}_{2}\right)$ & $(0.8)$ & $(1.2)$ & $(0.4)$ & & 0.2 \\
Untreated PF & 16.1 & 81.8 & & 2.2 & \\
& $(0.3)$ & $(0.1)$ & & $(0.2)$ & \\
Untreated PF + & 42.8 & 46.8 & 9.9 & 0.4 & \\
PMMA/ATH & $(1.4)$ & $(1.8)$ & $(0.6)$ & $(0.3)$ & \\
Coating C & 34.0 & 57.3 & 5.4 & 3.3 & 0.6 \\
(PMMA/ATH + PF in air) & $(0.4)$ & $(0.6)$ & $(0.2)$ & $(0.3)$ & \\
\hline
\end{tabular}

In summary, two modifications of coatings based on PMMA/ATH waste powder were deposited by cold plasma spraying. The replacement of air as process and carrier gas by 
forming gas led to a distinct depolymerisation of PMMA but failed to ensure particle fixation. On the other hand, the addition of PF powder was significantly able to enhance the bonding. SEM measurements showed film formation, but also piling of particles and agglomerates. The resulting spatial distances between the particles within the coating is assumed to prevent further improvement of the bonding within the coatings. In contrast, the adhesion between the coating and the substrates appeared to be strong. This is assumed to result from surface interactions between the hydroxyl groups of the resin and the wood constituents or the Si-OH groups, respectively. The hydrophobicity of the coatings suggests the application of further optimised coatings - for example, on wood - to reduce water uptake.

\subsection{Discussion}

As shown by the results summarised in Chapter 5.1 and 5.2, cold plasma spraying is an eligible technique to deposit PMMA/ATH powder-based coatings on wood and glass surfaces. The addition of PF to the pure PMMA/ATH powder permitted the generation of hydrophobic coatings and significantly increased the bonding, particularly between the layer and the substrates. Adhesion within the coating between individual particles/agglomerates needs further improvement and might be achieved by adjusting the deposition parameters or additionally reducing the particle sizes within the PMMA/ATH powder prior to deposition. Pure PMMA/ATH was found to be UV-stable [127]; however, coatings comprising PF underwent discolouration when exposed to UV light for $5 \mathrm{~h}$ in preliminary tests. Since hydrophobicity was preserved and the mechanical stability as assessed by abrasion tests did not change, this problem is assumed to be merely of an aesthetic nature. The addition of suitable pigments might ensure colour stability for an outdoor application.

The method chosen to evaluate the coatings' adhesion was based on the standard ISO 2409:2013 [173], but modified to suit the aim of the specific investigations. By gradually removing the particle coatings, studies on the bonding at different positions within the coatings and between the coatings and the substrates were possible in contrast to standard procedures $[173,174]$. However, since the tests did not precisely follow a standard, comparisons with results from other research groups were not possible. So far, only relative comparisons were of interest in order to evaluate the suitability of the tested approaches. In further studies, the tests could now be extended to the application of standard methods for coatings involving PF to achieve comparability. Moreover, the abrasion tests followed a standard usually applicable for optical coatings [175] because the standard methods for wood coatings, e.g. [176], were assumed to be too rigorous for the coatings featuring insufficient 
abrasion resistance. The abrasion tests could now also be extended to standard procedures for coatings involving PF powder in order to provide comparability with results from other research groups.

Studies on the chemical properties of the coatings by XPS revealed valuable information on the composition and consequently on the processes occurring in the plasma. For instance, even though the use of forming gas did not improve particle fixation, severe changes in the carbonic structure were shown. A transfer of the method might be of general use for other applications, probably to optimise the surface properties of other organic polymers. However, further studies on model substances or the application of further, complementary measurement techniques would be needed to expand the information on the chemical composition of the different coating systems. In the XPS spectra, the carbon peak included manifold overlapping subpeaks accounting for different carbon bonds and thus, sound spectral deconvolution was not possible without summing up several different subpeaks. Future studies could include other methods, for example MIES and UPS [50], to permit a further differentiation between the carbonic bonds involved. Moreover, the deposition of pure PF powder could be studied. However, since the choice of a specific powder generally seemed to influence the conditions in the effluent plasma, the comparability would therefore remain limited.

The adhesion and abrasion resistance of PMMA/ATH and PF coatings might be improved by optimising the deposition parameters. However, results from other types of particle coatings showed that the piling of particles presents a significant problem. For this reason, testing other approaches to ensure particle fixation appears worthwhile. Since PF seemed to undergo distinct film formation in the plasma, an application of a pure PF coating on top of the PMMA/ATH and PF coating could be tested. In a different approach, pure PMMA/ATH coatings were deposited with air as process and carrier gas. These coatings exhibited strongly hydrophilic properties, probably by the exposure of ATH and oxidation of PMMA. An increase in the hydrophilicity of PMMA by plasma treatments was also observed by several other research groups $[177,178]$. These changes may improve the bondability to polar topcoats like conventional waterborne varnishes, which could be applied following the plasma coating to ensure sealing. Moreover, suitable liquid monomers like MMA could be spread on the basic plasma coating and subjected to a second plasma treatment to evoke plasma polymerisation. To avoid the need for an additional processing step, monomers could also be injected into the effluent plasma concurrently with the PMMA/ATH powder. Preliminary tests were carried out with MMA, but even though the polymerisation of MMA is generally 
possible in a suitable plasma $[179,180]$, no film formation could be achieved by introducing the monomer into the effluent plasma from cold plasma spraying. Nevertheless, this approach could be further tested by modifying the deposition conditions - for example by exchanging the process and carrier gas. The introduction of HMDSO (see Chapter 4.4) did not lead to scratch resistant coatings; nonetheless, the combined deposition of PMMA/ATH and HMDSO was tested to study possible synergetic effects. As expected, however, no improvement of abrasion resistance was observed. As already mentioned in Chapter 4.4, a modification of the setup to permit injection of HMDSO very close to the substrate's surface could improve the deposition.

To summarise, depositing PMMA/ATH powder by cold plasma spraying appeared to be a viable approach to recycling a valuable industrial waste powder. Particle fixation could partly be achieved by adding a PF resin to the PMMA/ATH powder. Future work could further optimise the coatings' bonding and complement the characterisation of the layers. 


\section{Summary and Outlook}

The present thesis summarises studies about the properties of two different particle coatings deposited by cold plasma spraying on wood and glass substrates with a targeted application as protective layers. On the one hand, coatings consisting of zinc and zinc oxide aimed at decreasing photodegradation and fungal infestation of wood were studied. On the other hand, coatings based on a powder made of poly(methyl methacrylate) filled with aluminium trihydrate were investigated. Here, the starting point was the search for adequate recycling options of this waste powder in consideration of the material's protective potential.

The characterisation of the coatings with elemental zinc as the base material on homogeneous glass substrates confirmed the presence of a significant share of zinc oxide. Even though the coating was designed to yield an almost transparent layer, distinct UV-blocking properties were evident. Despite the use of rather large zinc particles as the starting material $\left(\mathrm{d}_{50}=13 \mu \mathrm{m}\right)$, topographic analysis revealed the generation of nano-scale structures. XPS measurements confirmed significant oxidation of the zinc powder. Hence, an application of the coatings to reduce degradation of wood by sunlight or microbial attack appeared conceivable. Studies on the impact of such coatings on the growth of blue stain, however, did not show any improvement. Presumably, the initial particle concentration was not sufficient. Leaching of the particles became apparent, leading to a further reduction of the particle concentration. Moreover, there was an insufficient homogeneity of large-scale coatings. Based on these results, the coatings were optimised for further studies. Artificial ageing tests were carried out with significantly increased particle concentrations and a zinc-base powder of a different particle geometry. Moreover, coating systems consisting of a particle coating and a conventional sealing were investigated. All samples that were covered with a coating system comprising a particle layer exhibited significantly less discolouration upon exposure to UV light. Nevertheless, chemical analysis revealed a photocatalytic degradation of the alkyd sealing in the presence of a particle coating. PUR-sealed samples, on the other hand, did not undergo chemical changes. Therefore, a coating system generated by the sequential application of a particle coating and the PUR used in this study provides a promising candidate as a layer to protect wood from photodegradation. In this way, a precise dosage of UV absorbers should be possible with the simultaneous fixation of the particles to prevent (nano-)particle release due to leaching or abrasion by windblown sand particles.

To develop such coatings further, future studies could investigate the efficacy of sealings with regard to unintentional particle release. Due to the photocatalytic activity of zinc oxide, the 
possibility of wood surface decomposition in the presence of such particle coatings needs to be investigated. With a thorough method to assess particle concentrations, detailed studies on the resulting particle load would be needed to study its dependence on the deposition parameters. To further increase the efficacy of the coatings, studies with base powders featuring a diameter in the nanometre-range could be carried out. In this work, elemental zinc was used as the base material because it was expected to melt easily during the deposition process and, due to subsequent solidification on the surface, to adhere well to the surface through micromechanical clamping. However, the adhesion was not sufficient to prevent leaching, as ascertained in the course of these studies, thus revealing the need for additional sealing. Another approach would be to deposit $\mathrm{ZnO}$ directly. Even though a melting would not be expected, electroadhesion is conceivable to occur and could yield a temporary fixation. Permanent fixation could then be achieved by subsequent sealing, for example, with a polyurethane. In this way, the necessary particle concentration could probably be decreased, since the zinc should be completely oxidised - thereby presumably permitting coatings that preserve the wood's natural appearance.

On the other hand, coatings based on PMMA/ATH powder deposited on glass and wood were investigated. In a first approach, the powder was deposited without additives and by using air as process and carrier gas. The characterisation of such coatings revealed an etching and slight oxidation of the PMMA matrix along with exposure of ATH filler particles. In addition to the high surface roughness, these chemical changes led to a hydrophilicity of the coatings and thus suggested a suitability to bond to polar top coats. No evidence for film-forming processes was found and accordingly, abrasion tests confirmed the need for particle fixation. Attempting to increase the coatings' mechanical stability, the process gas was replaced by forming gas. XPS affirmed severe depolymerisation, but these changes failed to ensure particle fixation. In contrast, the addition of phenol-formaldehyde powder to the PMMA/ATH and the use of air as process gas yielded improved bonding within the coating between individual particles/agglomerates and outstanding adhesion between the coating and the substrate, presumably by generating covalent bonds between the PF's hydroxyl groups and functional groups on the substrates' surfaces. Studies on the morphology and the chemical composition suggested an encasement of the PMMA/ATH particles by reacted PF, which additionally underwent film formation and thereby fused individual particles.

To further optimise the bonding of such coatings, several approaches as already discussed in Chapter 5.3 could be (further) tested. Accordingly, the deposition conditions for creating PMMA/ATH coatings could be adjusted with the aim to reduce piling of the particles on the 
substrate's surface and thus reduce the large spatial distances within the layers. Moreover, pure PMMA/ATH coatings could be sealed with a top-coat, generated for example by making use of the hydrophilic nature of such coatings and applying a conventional waterborne varnish, or by spreading a suitable monomer on the PMMA/ATH coating and causing polymerisation in a second, sequential plasma treatment. Moreover, a concurrent injection of PMMA/ATH powder and suitable monomers into the effluent plasma in cold plasma spraying could be further studied.

The PMMA/ATH and PF coatings were found to be very hydrophobic and thus provide a candidate as a coating to reduce the water uptake by wood. Since hydrophobic but porous coatings may reduce the uptake of liquid water but could fail to be a moisture barrier, future studies could investigate the protective properties of such coatings with regard to moisture uptake by wood. Moreover, many of the PMMA/ATH bulk material's properties discussed in Chapter 2.4 are favourable to wood protection. Further studies could concentrate on testing whether some of these properties could be transferred to the coatings and therefore meet requirements to protect wooden surfaces from various degradation factors.

In general, the elucidation of adhesion mechanisms between different powder coatings and substrates remains crucial. Results from the present work suggest micromechanical clamping as the dominant adhesion mechanism for the $\mathrm{Zn} / \mathrm{ZnO}$ coatings and the formation of covalent bonds for the PMMA/ATH and PF coatings. However, further studies on the interface between a coating and a substrate would be needed to support the hypotheses and elucidate other possible effects such as electroadhesion. Moreover, a sound characterisation of the coating facility would be needed to study the processes occurring in the plasma further. This characterisation could include spectroscopic techniques to assess information on different plasma temperatures and to identify the predominant species.

To summarise, the results elaborated within this thesis suggest that cold plasma spraying is a versatile tool for depositing different types of (protective) coatings on wood and glass surfaces. Based on these findings, several different approaches were discussed which could further optimise the coating systems to ensure a thorough protection of various surfaces, particularly in outdoor use. 


\section{Appendix - Hydrophobic Plasma Polymers}

In an attempt to identify potential sealings that inhibit an unintentional particle release and concurrently protect wood from water uptake, plasma polymers were generated by the polymerisation of ethylene (unpublished results). As intended, the created coatings on wood and glass featured highly hydrophobic properties. Studies on the optical, chemical, and morphologic properties as well as on resulting film thicknesses were carried out.

As discussed in Chapter 2.1.2, the plasma technology represents a versatile tool to create hydrophobic coatings, for example by plasma polymerisation of various precursors. Some approaches used ethylene $\left(\mathrm{C}_{2} \mathrm{H}_{4}\right)$ to generate coatings similar to polyethylene $[20,21,181,182]$. Based on these results, a DBD plasma was used to explore the eligibility of such coatings as hydrophobic layers which might eventually ensure particle fixation. The following chapter summarises the results which are described in more detail in [183].

\section{Experimental setup}

The configuration used to deposit coatings through the plasma polymerisation of ethylene is sketched in Figure 9. Two electrodes were located on opposite sides of a rectangular tube made of fused silica, with the sample placed in between. The rectangular tube ensured encapsulation and acted, together with the sample, as dielectric. A mixture of argon and ethylene is streamed through this tube and ionised and polymerised by a pulsed, alternating high voltage.

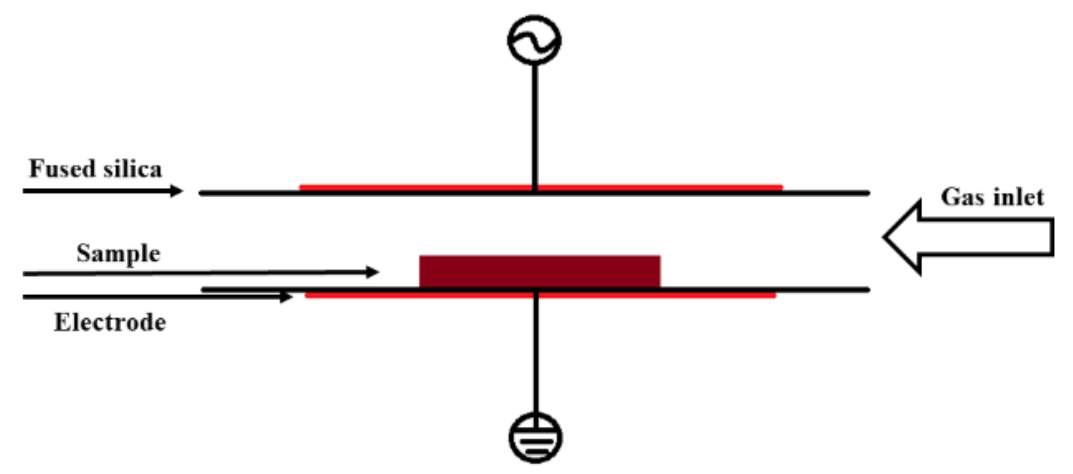

Figure 9: Se tup to create plasma polymers based on ethylene.

The basic setup permits manifold variations to the plasma parameters which crucially impact the physical and chemical properties of the resulting polymer [59,184,185]. To reduce complexity, the total gas flow and the discharge gap were kept constant at $6 \mathrm{~L} / \mathrm{min}$ and 
$9.8 \mathrm{~mm}$, respectively. By varying the applied voltage, the treatment time and the mixing ratio of argon and ethylene, the coatings were optimised with respect to hydrophobicity (see below). Details on the resulting input powers and energy densities can be found in [183].

\section{Wettability}

To assess the hydrophobicity of coated wood (beech, spruce) and thereby optimise the plasma parameters, water contact angles were measured and evaluated according to Nussbaum[186]. Since wood is a porous and soaking material, this method provides for the determination of constant wetting rate angles (CWRA) at which the spreading stage changes into constant penetration, characterised by the constancy of the angles' first derivative with time. As all coated samples featured constant contact angles, the application of this method was actually only important for untreated samples.

In the first step, only the treatment time was varied while keeping the mixing ratio constant at 2:1 $\left(\right.$ Ar: $\left.\mathrm{C}_{2} \mathrm{H}_{4}\right)$ and the outgoing voltage at $22 \mathrm{kV}$. According to the results displayed in Figure 10, the CWRAs for both beech and spruce initially increased with increasing treatment time, but remained constant for durations of $5 \mathrm{~s}$ or longer. Therefore, $5 \mathrm{~s}$ should be the optimal treatment time and was kept constant during further studies.

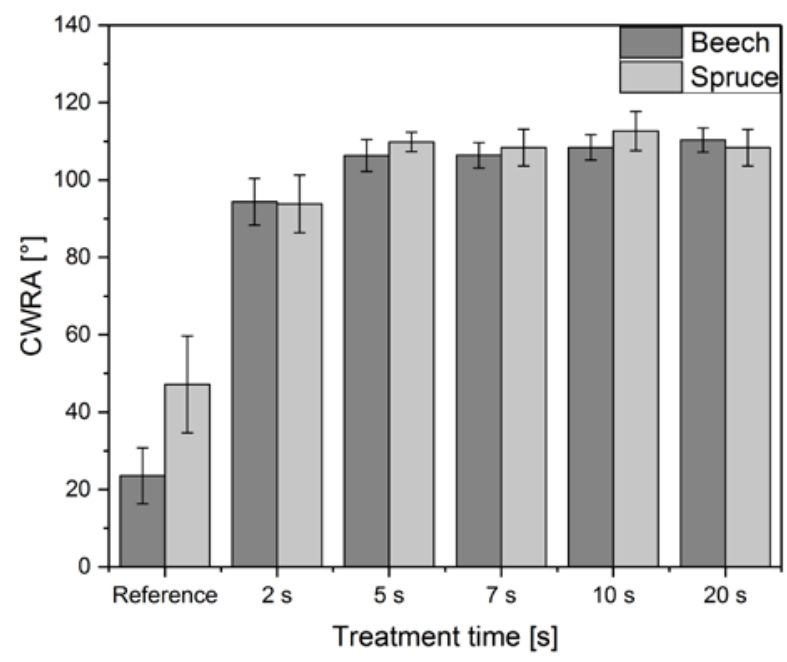

Figure 10: Evolution of the constant wetting rate angles (CWRA) with treatment time for coated beech and spruce.

Moreover, the mixing ratio and the input power were varied to some extent. For further analysis, the plasma parameters of the coatings yielding the highest CWRA were mainly used $\left(120.5^{\circ}\right.$ for beech and $121.6^{\circ}$ for spruce with a mixing ratio $\mathrm{Ar}: \mathrm{C}_{2} \mathrm{H}_{4}$ of 5:1 and an output voltage of $24 \mathrm{kV}$ ). In some cases, also coatings with the parameters leading to the lowest CWRA were studied $\left(114.9^{\circ}\right.$ for beech and 110.5 for spruce with a mixing ratio $\mathrm{Ar}: \mathrm{C}_{2} \mathrm{H}_{4}$ of 
$2: 1$ and an output voltage of $22 \mathrm{kV}$ ). Toriz et al. [21] even obtained contact angles of almost $140^{\circ}$; therefore, additional optimisation of the plasma parameters might further improve hydrophobicity.

\section{Aspects of optical properties and film thickness}

The film thickness and growth of the coatings that led to the highest contact angles were studied by ellipsometry ${ }^{26}$. For this purpose, multiple coatings were deposited on microscope slides. Since a change in the substrate material and a different discharge gap may distinctly change the plasma, the microscope slides were positioned on beech wood samples with a thickness reduced by $1 \mathrm{~mm}$ compared to the previous studies in order to keep the conditions in the plasma as constant as possible. The resulting film thicknesses for increasing numbers of coatings are depicted in Figure 11. For each number of coatings, three replicates were studied.

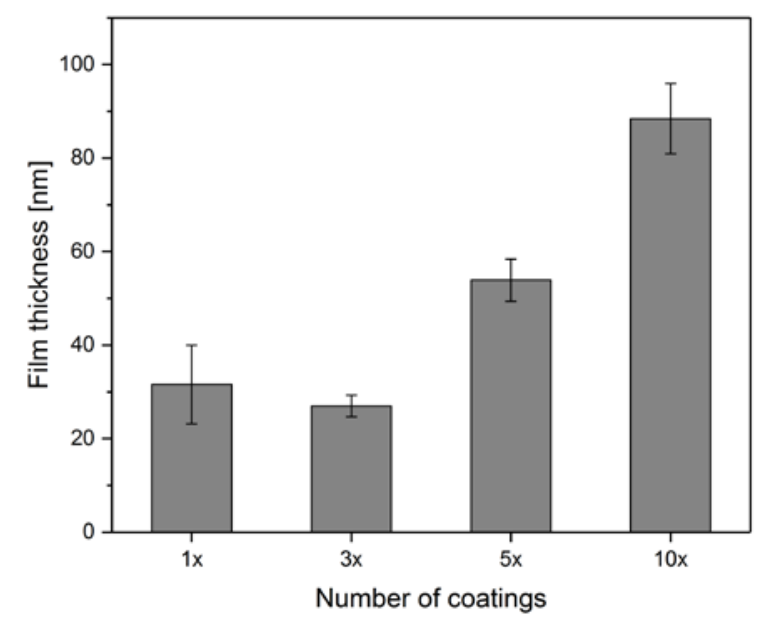

Figure 11: Evolution of film thickness with multiple coatings on glass.

The measurements indicated that the coatings did not grow linearly. Particularly the high thickness of single-coated samples compared to triple coatings was unexpected, but the resulting behaviour can be explained by topographic measurements (AFM) and is discussed below. Since determining film thicknesses in the nanometre range is challenging, no second method to validate the thicknesses was available. Instead, validation was carried out by comparing the resulting real part of the coatings' refractive indices. Refractive indices $\mathrm{n}$ can be determined from the same ellipsometric measurements, but also by other techniques such as UV-Vis spectroscopy. Therefore, the real part of the refractive indices as determined by

26 The execution and evaluation of ellipsometric measurements by Christian Röhling, Accurion GmbH, is gratefully acknowledged. 
ellipsometry and by UV-Vis spectroscopy were compared at a wavelength of $550 \mathrm{~nm}$. The results are summarised in Table 5.

Table 5: Comparis on of the refractive indices at $550 \mathrm{~nm}$ determined by ellipsometry and UV-Vis spectroscopy. Errors are given in parentheses.

\begin{tabular}{lccccc}
\hline Number of coatings: & $\mathbf{0 x}$ & $\mathbf{1} \mathbf{x}$ & $\mathbf{3} \mathbf{x}$ & $\mathbf{5 x}$ & $\mathbf{1 0} \mathbf{x}$ \\
\hline $\mathrm{n}_{\text {ellipsometry }}$ & 1.520 & 1.509 & 1.526 & 1.535 & 1.541 \\
& $(0.002)$ & $(0.01)$ & $(0.005)$ & $(0.006)$ & $(0.007)$ \\
$\mathrm{n}_{\text {UV-Vis }}$ & 1.524 & 1.510 & 1.526 & 1.543 & 1.569 \\
& $(0.001)$ & $(0.006)$ & $(0.008)$ & $(0.007)$ & $(0.015)$ \\
$\Delta \mathrm{n}$ & 0.004 & 0.001 & 0.000 & 0.008 & 0.028
\end{tabular}

Except for the samples with ten coatings, the resulting real parts of the refractive indices as assessed by the two methods differed only slightly. The values for the uncoated samples almost coincided with the literature value of $1.53 \pm 0.02$ at $546.07 \mathrm{~nm}$ [187]. Therefore, the film thicknesses determined from the same ellipsometric measurements as the refractive indices are assumed to be reliable.

\section{Topography}

To study the coatings' topography and assess if the irregular results for the film growth were caused by morphologic issues, AFM measurements were carried out. Representative measurements for samples coated $1 \mathrm{x}, 3 \mathrm{x}$, and $5 \mathrm{x}$ are displayed in Figure 12. Moreover, results from the determined roughness values $\mathrm{Ra}$ (i.e. the arithmetical mean deviation) are summarised in Table 6.

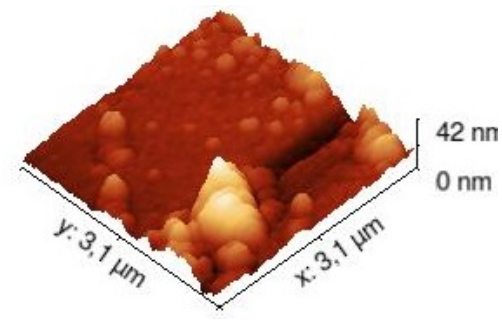

$1 \mathrm{x}$

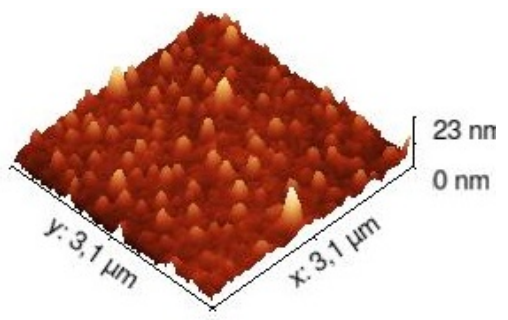

$3 x$

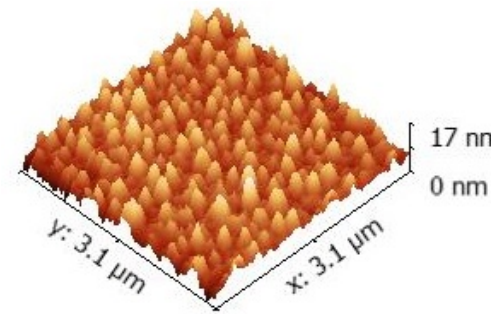

$5 \mathrm{x}$

Figure 12: Topography of coated microscope slides.

Table 6: Roughness parameter Ra of samples with multiple coatings.

\begin{tabular}{lccccc}
\hline Number of coatings: & $\mathbf{0} \mathbf{x}$ & $\mathbf{1 x}$ & $\mathbf{3 x}$ & $\mathbf{5 x}$ & $\mathbf{1 0} \mathbf{x}$ \\
\hline \multirow{2}{*}{$\mathrm{xa}[\mathrm{nm}]$} & 1.5 & 6.3 & 3.1 & 2.1 & 3.2 \\
& $(0.7)$ & $(2.5)$ & $(2.0)$ & $(0.7)$ & $(1.8)$ \\
\hline
\end{tabular}


Particularly for the samples coated only once, the measurements revealed a high surface roughness and many isolated peaks. During polymerisation, the coating therefore did not seem to be deposited homogeneously. For an increased number of coatings, the peaks appeared to be etched in the plasma and the spaces in between seemed to be filled, leading to increased homogeneity. These findings can therefore explain the results from film thickness measurements. Presumably, the film thickness measurements partly assessed the height of the isolated peaks and therefore yielded a higher film thickness for single-coated samples than for samples with triple coatings.

\section{Chemical composition}

Infrared analysis was performed to study the coatings' chemical composition. Since the low film thickness posed challenges regarding the acquisition of spectra, several different techniques were tested [183]. Eventually, 30 coatings were applied to one microscope slide. The layer material of several replicates was scratched off and mixed with $\mathrm{KBr}$ to form a pressed pellet, suitable for acquiring transmittance spectra. Relevant regions of the spectrum for the coating deposited with the plasma parameters that led to the highest contact angles are depicted in Figure 13.

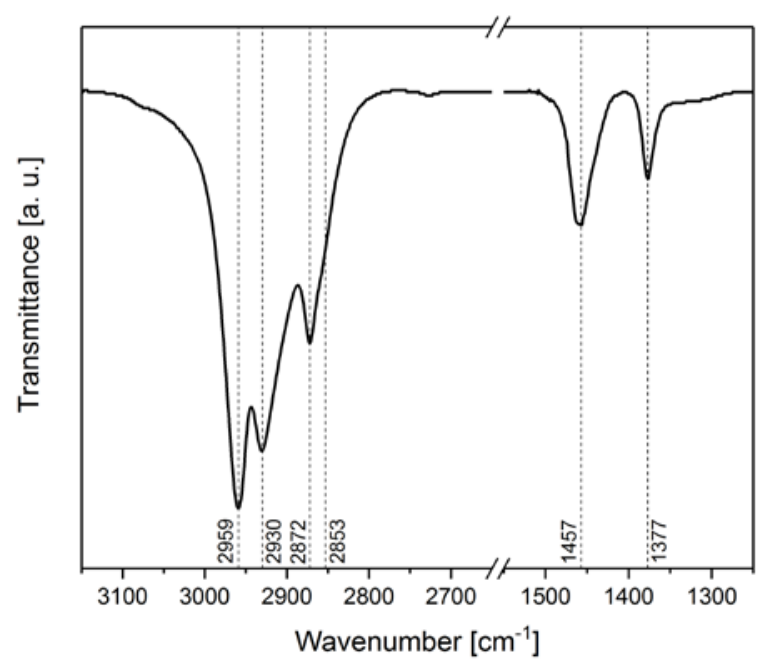

Figure 13: IR spectrum of the coating corresponding to the plasma parameters of the highest WCA.

The peaks at $2959 \mathrm{~cm}^{-1}, 2872 \mathrm{~cm}^{-1}$ and $1377 \mathrm{~cm}^{-1}$ account for contributions from $\mathrm{CH}_{3}$ bonds, peaks at $2930 \mathrm{~cm}^{-1}$ and $2853 \mathrm{~cm}^{-1}$ represent $\mathrm{CH}_{2}$ bonds, and the peak at $1457 \mathrm{~cm}^{-1}$ results from both methyl and methylene groups [188]. Compared to classical polyethylene, the peaks corresponding to $\mathrm{CH}_{3}$ groups are far more intense [188], indicating the generation of very short but presumably cross-linked polymer units. Deconvolution of the peaks was carried out 
to determine the ratio of $\mathrm{CH}_{3}: \mathrm{CH}_{2}$ for the parameters leading to the highest and the lowest contact angles [183], but these results need further validation. Preliminary analysis suggested a slightly higher share of $\mathrm{CH}_{3}$ groups for the plasma parameters, leading to the highest contact angles.

\section{Discussion}

By polymerising ethylene in a DBD-plasma, highly hydrophobic coatings could be generated. Studies on the film thickness revealed the generation of very thin layers in the nanometre range which acquired quite high homogeneity upon multiple coatings. Therefore, the eligibility of such coatings as moisture barriers might be studied to investigate if the hydrophobic coatings also reduce water and water vapour uptake.

The resulting film thickness in the nanometre range might be problematic with regard to an application as a particle sealing. Even though the coatings appeared to adhere well to glass surfaces as discovered when scraping off layer material to prepare $\mathrm{KBr}$ pellets, a successful stabilisation of the particle coatings in the micrometre range seems questionable. Leaching of such coating systems would need to be investigated carefully. Moreover, it became apparent that a proper encapsulation of the discharge area was crucial for generating hydrophobic coatings from ethylene. Slight leakiness already sufficed to introduce air and moisture, leading instead to the creation of hydrophilic layers. However, the need for encapsulation strongly limits the conceivable sample dimensions and thus prevents an eventual application on an industrial scale.

Even though the application of this method as described above is not considered to yield coatings suitable as hydrophobic layers on wood that additionally ensure particle fixation, it could be used to coat different substrate materials with hydrophobic layers that usually feature smaller dimensions. In addition, a modification of the setup could permit a better scalability and maybe even the generation of thicker layers, since the resulting plasma polymers strongly depend on the deposition parameters, including the plasma source's geometry $[59,184,185]$. 


\section{References}

[1] D. N.-S. Hon and S.-T. Chang, "Surface degradation of wood by ultraviolet light," Journal of Polymer Science: Polymer Chemistry Edition, vol. 22, no. 9, pp. 22272241, 1984.

[2] M. Shimada and M. Takahashi, "Biodegradation of cellulosic materials," in Wood and Cellulosic Chemistry, D. N.-S. Hon and N. Shiraishi, Eds., Marcel Dekker, New York, 1991.

[3] T. K. Kirk and E. B. Cowling, "Biological decomposition of solid wood," in The Chemistry of Solid Wood, R. Rowell, Ed., vol. 207, pp. 455-487, American Chemical Society, 1984.

[4] W. C. Feist and D. N.-S. Hon, "Chemistry of weathering and protection," in The Chemistry of Solid Wood, R. Rowell, Ed., American Chemical Society, 1984.

[5] B. George, E. Suttie, A. Merlin et al., "Photodegradation and photostabilisation of wood - the state of the art," Polymer Degradation and Stability, vol. 88, no. 2, pp. 268-274, 2005.

[6] D. N.-S. Hon, "Photochemistry of wood," in Wood and Cellulosic Chemistry, D. N.-S. Hon and N. Shiraishi, Eds., Marcel Dekker, New York, 1991.

[7] R. Rowell and M. Dietenberger, "Thermal properties, combustion, and fire retardancy of wood," in Handbook of Wood Chemistry and Wood Composites, R. Rowell, Ed., pp. 127-150, CRC Press, 2012.

[8] H. Derbyshire and E. R. Miller, "The photodegradation of wood during solar irradiation," European Journal of Wood and Wood Products, vol. 39, no. 8, pp. 341350, 1981.

[9] R. A. Blanchette, "A review of microbial deterioration found in archaeological wood from different environments," International Biodeterioration \& Biodegradation, vol. 46, no. 3, pp. 189-204, 2000.

[10] T. P. Schultz, D. D. Nicholas, and A. F. Preston, "A brief review of the past, present and future of wood preservation," Pest Management Science, vol. 63, no. 8, pp. 784$788,2007$.

[11] H. Militz and M. Altgen, "Processes and properties of thermally modified wood manufactured in Europe," in Deterioration and Protection of Sustainable Biomaterials, T. P. Schultz, B. Goodell, and D. D. Nicholas, Eds., pp. 269-285, American Chemical Society, 2014.

[12] A. Wolkenhauer, G. Avramidis, Y. Cai et al., "Investigation of wood and timber surface modification by dielectric barrier discharge at atmospheric pressure," Plasma Processes and Polymers, vol. 4, S1, 470-474, 2007.

[13] G. Avramidis, H. Militz, I. Avar et al., "Improved absorption characteristics of thermally modified beech veneer produced by plasma treatment," European Journal of Wood and Wood Products, vol. 70, no. 5, pp. 545-549, 2012.

[14] R. Wascher, N. Schulze, G. Avramidis et al., "Increasing the water uptake of wood veneers through plasma treatment at atmospheric pressure," European Journal of Wood and Wood Products, vol. 72, no. 5, pp. 685-687, 2014. 
[15] L. Podgorski, B. Chevet, L. Onic et al., "Modification of wood wettability by plasma and corona treatments," International Journal of Adhesion and Adhesives, vol. 20, no. 2, pp. 103-111, 2000.

[16] C. Lux, Z. Szalay, W. Beikircher et al., "Investigation of the plasma effects on wood after activation by diffuse coplanar surface barrier discharge," European Journal of Wood and Wood Products, vol. 71, no. 5, pp. 539-549, 2013.

[17] W. Viöl, G. Avramidis, and H. Militz, "Plasma treatment of wood," in Handbook of Wood Chemistry and Wood Composites, R. Rowell, Ed., pp. 627-658, CRC Press, 2012.

[18] H. Yasuda, "Glow discharge polymerization," Journal of Polymer Science: Macromolecular Reviews, vol. 16, no. 1, pp. 199-293, 1981.

[19] L. Podgorski, C. Bousta, F. Schambourg et al., "Surface modification of wood by plasma polymerisation," Pigment \& Resin Technology, vol. 31, no. 1, pp. 33-40, 2002.

[20] M. Bente, G. Avramidis, S. Förster et al., "Wood surface modification in dielectric barrier discharges at atmospheric pressure for creating water repellent characteristics," Holz als Roh- und Werkstoff, vol. 62, no. 3, pp. 157-163, 2004.

[21] G. Toriz, M. G. Gutiérrez, V. González-Alvarez et al., "Highly hydrophobic wood surfaces prepared by treatment with atmospheric pressure dielectric barrier discharges," Journal of Adhesion Science and Technology, vol. 22, no. 16, pp. 2059 2078, 2008.

[22] S. Zanini, C. Riccardi, M. Orlandi et al., "Wood coated with plasma-polymer for water repellence," Wood Science and Technology, vol. 42, no. 2, pp. 149-160, 2008.

[23] J. Profili, O. Levasseur, A. Koronai et al., "Deposition of nanocomposite coatings on wood using cold discharges at atmospheric pressure," Surface and Coatings Technology, vol. 309, pp. 729-737, 2017.

[24] B. Poaty, B. Riedl, P. Blanchet et al., "Improved water repellency of black spruce wood surfaces after treatment in carbon tetrafluoride plasmas," Wood Science and Technology, vol. 47, no. 2, pp. 411-422, 2013.

[25] R. B. Heimann, Plasma-Spray Coating: Principles and Applications, Wiley-VCH, Weinheim, 2008.

[26] J. Ružbarský and A. Panda, Plasma and Thermal Spraying, Springer International Publishing, Cham, 2017.

[27] P. Gascón-Garrido, N. Mainusch, H. Militz et al., "Copper and aluminium deposition by cold-plasma spray on wood surfaces: effects on natural weathering behaviour," European Journal of Wood and Wood Products, vol. 75, no. 3, pp. 315-324, 2016.

[28] P. Gascón-Garrido, N. Mainusch, H. Militz et al., "Effects of copper-plasma deposition on weathering properties of wood surfaces," Applied Surface Science, vol. 366, pp. 112-119, 2016.

[29] P. Gascón-Garrido, M. F. Thévenon, N. Mainusch et al., "Siloxane-treated and copperplasma-coated wood: Resistance to the blue stain fungus Aureobasidium pullulans and the termite Reticulitermes flavipes," International Biodeterioration \& Biodegradation, vol. 120, pp. 84-90, 2017.

[30] P. Gascón-Garrido, Treatment of wood with siloxanes and metal micro-particle deposition using plasma technology, Dissertation, University of Göttingen, 2016. 
[31] C. A. Clausen, F. Green, and S. Nami Kartal, "Weatherability and leach resistance of wood impregnated with nano-zinc oxide," Nanoscale Research Letters, vol. 5, no. 9, pp. 1464-1467, 2010.

[32] A. Sirelkhatim, S. Mahmud, A. Seeni et al., "Review on zinc oxide nanoparticles: antibacterial activity and toxicity mechanism," Nano-Micro Letters, vol. 7, no. 3, pp. 219-242, 2015.

[33] R. B. Duggins and C. Ford, US 3847865: Use of alumina trihydrate in a polymethyl methacrylate article, 1974.

[34] C. Basaran, S. Nie, and C. S. Hutchins, "Time dependent behavior of a particle filled composite PMMA/ATH at elevated temperatures," Journal of Composite Materials, vol. 42, no. 19, pp. 2003-2025, 2008.

[35] C. Tendero, C. Tixier, P. Tristant et al., "Atmospheric pressure plasmas: a review," Spectrochimica Acta Part B: Atomic Spectroscopy, vol. 61, no. 1, pp. 2-30, 2006.

[36] U. Stroth, Plasmaphysik: Phänomene, Grundlagen, Anwendungen, Vieweg + Teubner, Wiesbaden, 2011.

[37] M. A. Lieberman and A. J. Lichtenberg, Principles of Plasma Discharges and Materials Processing, John Wiley \& Sons, Hoboken, New Jersey, 2005.

[38] R. Hippler, S. Pfau, and M. Schmidt, Low Temperature Plasma Physics: Fundamental Aspects and Applications, Wiley-VCH, Berlin, 2001.

[39] F. Peters, B. Hünnekens, S. Wieneke et al., "Comparison of three dielectric barrier discharges regarding their physical characteristics and influence on the adhesion properties on maple, high density fiberboards and wood plastic composite," Journal of Physics D: Applied Physics, 2017.

[40] U. Kogelschatz, "Dielectric-barrier discharges: their history, discharge physics, and industrial applications," Plasma Chemistry and Plasma Processing, vol. 23, no. 1, pp. 1-46, 2003.

[41] A. Wolkenhauer, G. Avramidis, E. Hauswald et al., "Sanding vs. plasma treatment of aged wood: A comparison with respect to surface energy," International Journal of Adhesion and Adhesives, vol. 29, no. 1, pp. 18-22, 2009.

[42] I. Sakata, M. Morita, N. Tsuruta et al., "Activation of wood surface by corona treatment to improve adhesive bonding," Journal of Applied Polymer Science, vol. 49, no. 7, pp. 1251-1258, 1993.

[43] R. Mahlberg, H. E.-M. Niemi, F. S. Denes et al., "Application of AFM on the adhesion studies of oxygen-plasma-treated polypropylene and lignocellulosics," Langmuir, vol. 15, no. 8, pp. 2985-2992, 1999.

[44] G. Avramidis, E. Hauswald, A. Lyapin et al., "Plasma treatment of wood and woodbased materials to generate hydrophilic or hydrophobic surface characteristics," Wood Material Science \& Engineering, vol. 4, 1-2, pp. 52-60, 2009.

[45] A. Wolkenhauer, G. Avramidis, E. Hauswald et al., "Plasma treatment of wood-plastic composites to enhance their adhesion properties," Journal of Adhesion Science and Technology, vol. 22, no. 16, pp. 2025-2037, 2008.

[46] B. Hünnekens, F. Peters, G. Avramidis et al., "Plasma treatment of wood-polymer composites: a comparison of three different discharge types and their effect on surface properties," Journal of Applied Polymer Science, vol. 133, no. 18, 2016. 
[47] G. Avramidis, L. Klarhöfer, W. Maus-Friedrichs et al., "Influence of air plasma treatment at atmospheric pressure on wood extractives," Polymer Degradation and Stability, vol.97, no. 3, pp. 469-471, 2012.

[48] G. Avramidis, R. Wascher, H. Militz et al., "Impact of air-plasma treatment at atmospheric pressure on wood and wood extractives," International Wood Products Journal, vol. 7, no. 2, pp. 76-79, 2016.

[49] L. C. Vander Wielen, M. Östenson, P. Gatenholm et al., "Surface modification of cellulosic fibers using dielectric-barrier discharge," Carbohydrate Polymers, vol. 65, no. 2, pp. 179-184, 2006.

[50] L. Klarhöfer, W. Viöl, and W. Maus-Friedrichs, "Electron spectroscopy on plasma treated lignin and cellulose," Holzforschung, vol. 64, no. 3, pp. 331-336, 2010.

[51] M. Odrášková, J. Ráhel, A. Zahoranová et al., "Plasma activation of wood surface by diffuse coplanar surface barrier discharge," Plasma Chemistry and Plasma Processing, vol. 28, no. 2, pp. 203-211, 2008.

[52] M. N. Belgacem, G. Czeremuszkin, S. Sapieha et al., "Surface characterization of cellulose fibres by XPS and inverse gas chromatography," Cellulose, vol. 2, no. 3, pp. 145-157, 1995.

[53] A. Jamali and P. D. Evans, "Etching of wood surfaces by glow discharge plasma," Wood Science and Technology, vol. 45, no. 1, pp. 169-182, 2011.

[54] P. Král, M. Stupavská, J. Šrajer et al., "XPS depth profile of plasma-activated surface of beech wood (Fagus sylvatica) and its impact on polyvinyl acetate tensile shear bond strength," Wood Science and Technology, vol. 49, no. 2, pp. 319-330, 2015.

[55] R. Wascher, G. Avramidis, U. Vetter et al., "Plasma induced effects within the bulk material of wood veneers," Surface and Coatings Technology, vol. 259, pp. 62-67, 2014.

[56] C. A. Clausen, "Biodeterioration of Wood," in Wood Handbook - Wood as an Engineering Material, Madison, Wis., 2010.

[57] N. Morosoff, "An introduction to plasma polymerization," in Plasma Deposition, Treatment, and Etching of Polymers, R. d'Agostino, Ed., pp. 1-93, Academic Press, San Diego, 1990.

[58] H. Yasuda and T. Hsu, "Plasma polymerization investigated by the comparison of hydrocarbons and perfluorocarbons," Surface Science, vol. 76, no. 1, pp. 232-241, 1978.

[59] H. Yasuda and T. Yasuda, "The competitive ablation and polymerization (CAP) principle and the plasma sensitivity of elements in plasma polymerization and treatment," Journal of Polymer Science Part A: Polymer Chemistry, vol. 38, no. 6, pp. 943-953, 2000.

[60] O. Levasseur, L. Stafford, N. Gherardi et al., "Deposition of hydrophobic functional groups on wood surfaces using atmospheric-pressure dielectric barrier discharge in helium-hexamethyldisiloxane gas mixtures," Plasma Processes and Polymers, vol. 9, 11-12, pp. 1168-1175, 2012.

[61] R. Mahlberg, H.-M. Niemi, F. Denes et al., "Effect of oxygen and hexamethyldisiloxane plasma on morphology, wettability and adhesion properties of polypropylene and lignocellulosics," International Journal of Adhesion and Adhesives, vol. 18, no. 4, pp. 283-297, 1998. 
[62] A. R. Denes, M. A. Tshabalala, R. Rowell et al., "Hexamethyldisiloxane-plasma coating of wood surfaces for creating water repellent characteristics," Holzforschung, vol. 53, no. 3, pp. 318-326, 1999.

[63] N. Gherardi, J. Profili, A. Koronai et al., "Synthesis of silica-like coatings containing metal-oxide nanoparticles by nitrogen-based dielectric barrier discharge," 22nd International Symposium on Plasma Chemistry, 2015.

[64] O. Beier, A. Pfuch, K. Horn et al., "Low temperature deposition of antibacterially active silicon oxide layers containing silver nanoparticles, prepared by atmospheric pressure plasma chemical vapor deposition," Plasma Processes and Polymers, vol. 10, no. 1, pp. 77-87, 2013.

[65] J. Profili, O. Levasseur, J.-B. Blaisot et al., "Nebulization of nanocolloidal suspensions for the growth of nanocomposite coatings in dielectric barrier discharges," Plasma Processes and Polymers, vol. 13, no. 10, pp. 981-989, 2016.

[66] M. S. Moghaddam, G. Heydari, M. Tuominen et al., "Hydrophobisation of wood surfaces by combining liquid flame spray (LFS) and plasma treatment: dynamic wetting properties," Holzforschung, vol. 70, no. 6, pp. 527-537, 2016.

[67] P. Rehn and W. Viöl, "Dielectric barrier discharge treatments at atmospheric pressure for wood surface modification," European Journal of Wood and Wood Products, vol. 61, no. 2, pp. 145-150, 2003.

[68] W. L. E. Magalhães and M. F. de Souza, "Solid softwood coated with plasma-polymer for water repellence," Surface and Coatings Technology, vol. 155, no. 1, pp. 11-15, 2002 .

[69] A. R. Denes and R. A. Young, "Reduction of weathering degradation of wood through plasma-polymer coating," Holzforschung, vol. 53, no. 6, pp. 632-640, 1999.

[70] H. Teisala, M. Tuominen, M. Aromaa et al., "Development of superhydrophobic coating on paperboard surface using the liquid flame spray," Surface and Coatings Technology, vol. 205, no. 2, pp. 436-445, 2010.

[71] J. M. Mäkelä, M. Aromaa, H. Teisala et al., "Nanoparticle deposition from liquid flame spray onto moving roll-to-roll paperboard material," Aerosol Science and Technology, vol. 45, no. 7, pp. 827-837, 2011.

[72] R. S. Williams, "Weathering of wood," in Handbook of Wood Chemistry and Wood Composites, R. Rowell, Ed., vol. 7, pp. 139-185, CRC Press, 2005.

[73] P. D. Evans, "Weathering of wood and wood composites," in Handbook of Wood Chemistry and Wood Composites, R. Rowell, Ed., vol. 2, pp. 151-216, CRC Press, 2012.

[74] "Solar energy; Reference solar spectral irradiance at the ground at different receiving conditions; Part 1: Direct normal and hemispherical solar irradiance for air mass 1,5," 1992, ISO 9845-1:1992.

[75] Y. Kataoka, M. Kiguchi, and P. D. Evans, "Photodegradation depth profile and penetration of light in japanese cedar earlywood (Cryptomeria japonica D. Don) exposed to artificial solar radiation," Surface Coatings International Part B: Coatings Transactions, vol. 87, no. 3, pp. 187-193, 2004.

[76] R. C. Petterson, "The chemical composition of wood," in The Chemistry of Solid Wood, R. Rowell, Ed., vol. 207, American Chemical Society, 1984. 
[77] R. M. Rowell, R. Pettersen, J. S. Han et al., "Cell wall chemistry," in Handbook of Wood Chemistry and Wood Composites, R. Rowell, Ed., vol. 2, CRC Press, 2005.

[78] D. N.-S. Hon, "Formation of free radicals in photoirradiated cellulose. VI. Effect of lignin," Journal of Polymer Science: Polymer Chemistry Edition, vol. 13, no. 12, pp. 2641-2652, 1975.

[79] C. A. S. Hill, Wood Modification: Chemical, Thermal and other Processes, John Wiley \& Sons, Chichester, 2007.

[80] P. D. Evans, A. J. Michell, and K. J. Schmalzl, "Studies of the degradation and protection of wood surfaces," Wood Science and Technology, vol. 26, no. 2, 1992.

[81] E. Adler, "Lignin chemistry - past, present and future," Wood Science and Technology, vol. 11 , no. 3, pp. 169-218, 1977.

[82] C. Kamoun, A. Merlin, X. Deglise et al., "Etude par spectroscopie de résonance paramagnétique électronique de la photodégradation des lignines extraites du bois de pin radiata (Pinus radiata D. Don)," Annals of Forest Science, vol. 56, no. 7, pp. 563$578,1999$.

[83] U. Müller, M. Rätzsch, M. Schwanninger et al., "Yellowing and IR-changes of spruce wood as result of UV-irradiation," Journal of Photochemistry and Photobiology B: Biology, vol. 69, no. 2, pp. 97-105, 2003.

[84] Kai, Y. U. J. I., "Chemistry of extractives," in Wood and Cellulosic Chemistry, D. N.S. Hon and N. Shiraishi, Eds., Marcel Dekker, New York, 1991.

[85] A. M. Taylor, B. L. Gartner, and J. J. Morrell, "Heartwood formation and natural durability-a review," Wood and Fiber Science, vol. 34, no. 4, pp. 587-611, 2007.

[86] P.-N. Diouf, A. Merlin, and D. Perrin, "Antioxidant properties of wood extracts and colour stability of woods," Annals of Forest Science, vol. 63, no. 5, pp. 525-534, 2006.

[87] F. Gugumus, "Current trends in mode of action of hindered amine light stabilizers," Polymer Degradation and Stability, vol. 40, no. 2, pp. 167-215, 1993.

[88] P. Hayoz, W. Peter, and D. Rogez, "A new innovative stabilization method for the protection of natural wood," Progress in Organic Coatings, vol. 48, no. 2, pp. 297 309, 2003.

[89] M. Vlad Cristea, B. Riedl, and P. Blanchet, "Enhancing the performance of exterior waterborne coatings for wood by inorganic nanosized UV absorbers," Progress in Organic Coatings, vol. 69, no. 4, pp. 432-441, 2010.

[90] Y. Yu, Z. Jiang, G. Wang et al., "Growth of ZnO nanofilms on wood with improved photostability," Holzforschung, vol. 64, no. 3, pp. 385-390, 2010.

[91] J. Salla, K. K. Pandey, and K. Srinivas, "Improvement of UV resistance of wood surfaces by using $\mathrm{ZnO}$ nanoparticles," Polymer Degradation and Stability, vol. 97, no. 4, pp. 592-596, 2012.

[92] S. M. Fufa, B. P. Jelle, and P. J. Hovde, "Effects of $\mathrm{TiO}_{2}$ and clay nanoparticles loading on weathering performance of coated wood," Progress in Organic Coatings, vol. 76, no. 10, pp. 1425-1429, 2013.

[93] W. B. Cooke and G. Matsuura, "Physiological studies in the black yeasts," Mycopathologia et Mycologia Applicata, vol. 21, 3-4, pp. 225-271, 1963. 
[94] M. W. Schoeman and D. J. Dickinson, “Aureobasidium pullulans can utilize simple aromatic compounds as a sole source of carbon in liquid culture," Letters in Applied Microbiology, vol. 22, no. 2, pp. 129-131, 1996.

[95] C. Mai, U. Kües, and H. Militz, "Biotechnology in the wood industry," Applied Microbiology and Biotechnology, vol. 63, no. 5, pp. 477-494, 2004.

[96] H. Militz and C. Mai, "Wood preservatives," in Wood Production, Wood Technology, and Biotechnological Impacts, U. Kües, Ed., Universitätsverlag Göttingen, 2007.

[97] C. A. Clausen, V. W. Yang, R. A. Arango et al., "Feasibility of nanozinc oxide as a wood preservative," Proceedings of American Wood Protection Association, vol. 105, pp. 255-260, 2009.

[98] S. N. Kartal, F. Green, and C. A. Clausen, "Do the unique properties of nanometals affect leachability or efficacy against fungi and termites?," International Biodeterioration \& Biodegradation, vol. 63, no. 4, pp. 490-495, 2009.

[99] M. Akhtari, H. R. Taghiyari, and M. G. Kokandeh, "Effect of some metal nanoparticles on the spectroscopy analysis of Paulownia wood exposed to white-rot fungus," European Journal of Wood and Wood Products, vol. 71, no. 2, pp. 283-285, 2013.

[100] R. H. Lamoreaux, D. L. Hildenbrand, and L. Brewer, "High-temperature vaporization behavior of oxides II. Oxides of Be, $\mathrm{Mg}, \mathrm{Ca}, \mathrm{Sr}, \mathrm{Ba}, \mathrm{B}, \mathrm{Al}, \mathrm{Ga}, \mathrm{In}, \mathrm{Tl}, \mathrm{Si}, \mathrm{Ge}, \mathrm{Sn}, \mathrm{Pb}$, Zn, Cd, and Hg," Journal of Physical and Chemical Reference Data, vol. 16, no. 3, pp. 419-443, 1987.

[101] E. N. Bunting, "Phase equilibria in the system $\mathrm{SiO}_{2}-\mathrm{ZnO}$," Journal of the American Ceramic Society, vol. 13, no. 1, pp. 5-10, 1930.

[102] I. Niikura and Y. Kashiwaba, "Crystal growth of bulk ZnO single crystal," in Wide Bandgap Semiconductors: Fundamental Properties and Modern Photonic and Electronic Devices, K. Takahashi, A. Yoshikawa, and A. Sandhu, Eds., pp. 356-362, Springer, Heidelberg, Berlin, 2007.

[103] D. E. Wurster, E. Oh, and J. C. Wang, "Determination of the mechanism for the decrease in zinc oxide surface area upon high-temperature drying," Journal of Pharmaceutical Sciences, vol. 84, no. 11, pp. 1301-1307, 1995.

[104] A. F. Holleman and E. Wiberg, Lehrbuch der anorganischen Chemie, W. de Gruyter, Berlin, New York, 1995.

[105] H. Morkoç and Ü. Özgür, Zinc Oxide, Wiley-VCH, Weinheim, 2009.

[106] J. Wang, Z. Wang, B. Huang et al., "Oxygen vacancy induced band-gap narrowing and enhanced visible light photocatalytic activity of $\mathrm{ZnO}$," ACS Applied Materials \& Interfaces, vol. 4, no. 8, pp. 4024-4030, 2012.

[107] P. L. Washington, H. C. Ong, J. Y. Dai et al., "Determination of the optical constants of zinc oxide thin films by spectroscopic ellipsometry," Applied Physics Letters, vol. 72, no. 25, pp. 3261-3263, 1998.

[108] E. A. Dalchiele, P. Giorgi, R. E. Marotti et al., "Electrodeposition of ZnO thin films on n-Si (100)," Solar Energy Materials and Solar Cells, vol. 70, no. 3, pp. 245-254, 2001.

[109] A. Mills and S. Le Hunte, "An overview of semiconductor photocatalysis," Journal of Photochemistry and Photobiology A: Chemistry, vol. 108, no. 1, pp. 1-35, 1997. 
[110] N. Serpone and A. V. Emeline, "Suggested terms and definitions in photocatalysis and radiocatalysis,” International Journal of Photoenergy, vol. 4, no. 3, pp. 91-131, 2002.

[111] M. M. Haque, D. Bahnemann, and M. Muneer, "Photocatalytic degradation of organic pollutants: mechanisms and kinetics," Organic Pollutants Ten Years After the Stockholm Convention-Environmental and Analytical Update, 2012.

[112] S. Sun, J. Ding, J. Bao et al., "Photocatalytic oxidation of gaseous formaldehyde on $\mathrm{TiO}_{2}$ : an in situ DRIFTS study," Catalysis Letters, vol. 137, no. 3, pp. 239-246, 2010.

[113] A. E. H. Machado, A. M. Furuyama, S. Z. Falone et al., "Photocatalytic degradation of lignin and lignin models, using titanium dioxide: the role of the hydroxyl radical," Chemosphere, vol. 40, no. 1, pp. 115-124, 2000.

[114] Y. Liao, C. Xie, Y. Liu et al., "Comparison on photocatalytic degradation of gaseous formaldehyde by $\mathrm{TiO}_{2}, \mathrm{ZnO}$ and their composite," Ceramics International, vol. 38, no. 6, pp. 4437-4444, 2012.

[115] C. H. Ao, S. C. Lee, J. Z. Yu et al., "Photodegradation of formaldehyde by photocatalyst $\mathrm{TiO}_{2}$ : effects on the presences of $\mathrm{NO}, \mathrm{SO}_{2}$ and VOCs," Applied Catalysis B: Environmental, vol. 54, no. 1, pp. 41-50, 2004.

[116] P. A. Christensen, A. Dilks, T. A. Egerton et al., "Photocatalytic oxidation of alkyd paint films measured by FTIR analysis of UV generated carbon dioxide," Journal of Materials Science, vol. 37, no. 22, pp. 4901-4909, 2002.

[117] J. Auvinen and L. Wirtanen, "The influence of photocatalytic interior paints on indoor air quality," Atmospheric Environment, vol. 42, no. 18, pp. 4101-4112, 2008.

[118] C. A. McDevitt, A. D. Ogunniyi, E. Valkov et al., "A molecular mechanism for bacterial susceptibility to zinc," PLoS Pathogens, vol. 7, no. 11, e1002357, 2011.

[119] S. B. Pointing, V. Bucher, and L. Vrijmoed, "Dye decolorization by sub-tropical basidiomycetous fungi and the effect of metals on decolorizing ability," World Journal of Microbiology and Biotechnology, vol. 16, no. 2, pp. 199-205, 2000.

[120] G. D. Savi, A. J. Bortoluzzi, and V. M. Scussel, "Antifungal properties of zinccompounds against toxigenic fungi and mycotoxin," International Journal of Food Science \& Technology, vol. 48, no. 9, pp. 1834-1840, 2013.

[121] L. He, Y. Liu, A. Mustapha et al., "Antifungal activity of zinc oxide nanoparticles against Botrytis cinerea and Penicillium expansum," Microbiological Research, vol. 166, no. 3, pp. 207-215, 2011.

[122] L. K. Adams, D. Y. Lyon, and P. J. J. Alvarez, "Comparative eco-toxicity of nanoscale $\mathrm{TiO}_{2}, \mathrm{SiO}_{2}$, and $\mathrm{ZnO}$ water suspensions," Water Research, vol. 40, no. 19, pp. 35273532, 2006.

[123] M. Khan, A. H. Naqvi, and M. Ahmad, "Comparative study of the cytotoxic and genotoxic potentials of zinc oxide and titanium dioxide nanoparticles," Toxicology Reports, vol. 2, pp. 765-774, 2015.

[124] T. Xia, M. Kovochich, M. Liong et al., "Comparison of the mechanism of toxicity of zinc oxide and cerium oxide nanoparticles based on dissolution and oxidative stress properties," ACS Nano, vol. 2, no. 10, pp. 2121-2134, 2008.

[125] K. Hirota, M. Sugimoto, M. Kato et al., "Preparation of zinc oxide ceramics with a sustainable antibacterial activity under dark conditions," Ceramics International, vol. 36, no. 2, pp. 497-506, 2010. 
[126] R. B. Duggins, C. Ford, H. C. Miller et al., US 3827933: Filled polymethyl methacrylate article and a process for its manufacture, 1974.

[127] A. Byrdy and M. Kołaczkowski, "Environmental impacts on the strength parameters of mineral-acrylic (PMMA/ATH) facade panels," International Journal of Polymer Science, vol. 2015, pp. 1-5, 2015.

[128] C. R. Peters and J. E. Young, US 6554944B1: Process for making bowls comprising acrylic plastics filled with alumina trihydrate, 2003.

[129] E. Šušteršič, M. Tušar, and A. Zupančič Valant, "Rheological and mechanical characterization of waste PMMA/ATH modified bitumen," Construction and Building Materials, vol. 38, pp. 119-125, 2013.

[130] S. Nie, C. Basaran, C. S. Hutchins et al., "Failure mechanisms in PMMA/ATH acrylic casting dispersion," Journal of the Mechanical Behavior of Materials, vol. 17, no. 2, pp. 79-96, 2006.

[131] O. Obakponovwe and J. G. Williams, "Temperature effects on the fatigue of highly filled PMMA," Journal of Materials Science, vol. 41, no. 2, pp. 437-443, 2006.

[132] A. P. Mouritz and A. G. Gibson, "Flame retardant composites," in Fire Properties of Polymer Composite Materials, A. P. Mouritz and A. G. Gibson, Eds., pp. 237-286, Springer, Dordrecht, 2006.

[133] K. Daimatsu, H. Sugimoto, Y. Kato et al., "Preparation and physical properties of flame retardant acrylic resin containing nano-sized aluminum hydroxide," Polymer Degradation and Stability, vol. 92, no. 8, pp. 1433-1438, 2007.

[134] J. Hochberg and R. B. Young, US 4759774 A: Process for cleaning substrates, 1988.

[135] W. Kaminsky, M. Predel, and A. Sadiki, "Feedstock recycling of polymers by pyrolysis in a fluidised bed," Polymer Degradation and Stability, vol. 85, no. 3, pp. 1045-1050, 2004.

[136] W. Kaminsky and J. Franck, "Monomer recovery by pyrolysis of poly(methyl methacrylate) (PMMA)," Journal of Analytical and Applied Pyrolysis, vol. 19, pp. 311-318, 1991.

[137] E. Šušteršič, A. Nikonov, and A. Zupančič Valant, "Influence of ageing on viscoelastic properties of PMMA/ATH modified bitumen," Polymer Composites, vol. 36, no. 9, pp. 1738-1747, 2015.

[138] E. Šušteršič, M. Tušar, and A. Zupančič Valant, "Asphalt concrete modification with waste PMMA/ATH,” Materials and Structures, vol. 47, no. 11, pp. 1817-1824, 2014.

[139] M. Tušar, E. Šušteršič, and A. Zupančič-Valant, "Alternative PMB produced from recycling waste PMMA/ATH," Transport Problems, vol. 9, no. 2, pp. 5-9, 2014.

[140] M. Tušar, A. Beličič, M. Prešeren et al., EP 20130730823: Modified bitumen and its use for preparing asphalt mixtures and bituminous products, 2015.

[141] D. Zhang, P. H. Shipway, and D. G. McCartney, "Cold gas dynamic spraying of aluminum: The role of substrate characteristics in deposit formation," Journal of Thermal Spray Technology, vol. 14, no. 1, pp. 109-116, 2005.

[142] I. Burlacov, J. Jirkovský, L. Kavan et al., "Cold gas dynamic spraying (CGDS) of $\mathrm{TiO}_{2}$ (anatase) powders onto poly (sulfone) substrates: Microstructural characterisation and photocatalytic efficiency," Journal of Photochemistry and Photobiology A: Chemistry, vol. 187, no. 2, pp. 285-292, 2007. 
[143] N. Bala, H. Singh, J. Karthikeyan et al., "Cold spray coating process for corrosion protection: a review," Surface Engineering, vol. 30, no. 6, pp. 414-421, 2013.

[144] E. Irissou, J.-G. Legoux, A. N. Ryabinin et al., "Review on cold spray process and technology: part I-intellectual property," Journal of Thermal Spray Technology, vol. 17, no. 4, pp. 495-516, 2008.

[145] A. Moridi, S. M. Hassani-Gangaraj, M. Guagliano et al., "Cold spray coating: review of material systems and future perspectives," Surface Engineering, vol. 30, no. 6, pp. 369-395, 2013.

[146] P. Fauchais, "Understanding plasma spraying," Journal of Physics D: Applied Physics, vol. 37, no. 9, R86-R108, 2004.

[147] L.-Y. Hsu and H.-M. Chein, "Evaluation of nanoparticle emission for $\mathrm{TiO}_{2}$ nanopowder coating materials," Journal of Nanoparticle Research, vol. 9, no. 1, pp. 157-163, 2007.

[148] P. H. M. Hoet, I. Brüske-Hohlfeld, and O. V. Salata, "Nanoparticles - known and unknown health risks," Journal of Nanobiotechnology, vol. 2, no. 1, p. 12, 2004.

[149] A. Baun, N. B. Hartmann, K. Grieger et al., "Ecotoxicity of engineered nanoparticles to aquatic invertebrates: a brief review and recommendations for future toxicity testing," Ecotoxicology, vol. 17, no. 5, pp. 387-395, 2008.

[150] B. V. Tata and B. Raj, "Confocal laser scanning microscopy: applications in material science and technology," Bulletin of Materials Science, vol. 21, no. 4, pp. 263-278, 1998.

[151] G. Binnig, C. F. Quate, and C. Gerber, "Atomic force microscope," Physical Review Letters, vol. 56, no. 9, pp. 930-933, 1986.

[152] G. H. Michler, Electron Microscopy of Polymers, Springer, Berlin, Heidelberg, 2008.

[153] J. F. Moulder, W. F. Stickle, P. E. Sobol et al., Handbook of X-Ray Photoelectron Spectroscopy: A Reference Book of Standard Spectra for Identification and Interpretation of XPS Data, Physical Electronics Division, Japan, 1992.

[154] D. A. Shirley, "High-resolution X-ray photoemission spectrum of the valence bands of gold," Physical Review B, vol. 5, no. 12, pp. 4709-4714, 1972.

[155] B. D. Ratner and D. G. Castner, "Electron Spectroscopy for Chemical Analysis," in Surface analysis: The principal techniques, J. C. Vickerman and I. S. Gilmore, Eds., pp. 47-112, John Wiley \& Sons, Hoboken, NJ, 2009.

[156] G. Ertl and J. Küppers, Low Energy Electrons and Surface Chemistry, VCH Verlagsgesellschaft, Weinheim, 1985.

[157] H. Günzler and H.-U. Gremlich, IR-Spektroskopie: Eine Einführung, Wiley-VCH, Weinheim, 2003.

[158] B. C. Smith, Fundamentals of Fourier Transform Infrared Spectroscopy, CRC Press, Boca Raton, FL, 2011.

[159] "Wood preservatives - Determination of the protective effectiveness of a treatment against blue stain in wood in service - Laboratory method," DIN EN 152:2012.

[160] V. Blanchard and P. Blanchet, "Color stability for wood products during use: effects of inorganic nanoparticles," BioResources, vol. 6, no. 2, pp. 1219-1229, 2011. 
[161] N. Auclair, B. Riedl, V. Blanchard et al., "Improvement of photoprotection of wood coatings by using inorganic nanoparticles as ultraviolet absorbers," Forest Products Journal, vol. 61, no. 1, pp. 20-27, 2011.

[162] M. R. M. Farahani and F. Banikarim, "Effect of nano-zinc oxide on decay resistance of wood-plastic composites," BioResources, vol. 8, no. 4, pp. 5715-5720, 2013.

[163] J. Miklečić, S. L. Blagojević, M. Petrič et al., "Influence of $\mathrm{TiO}_{2}$ and $\mathrm{ZnO}$ nanoparticles on properties of waterborne polyacrylate coating exposed to outdoor conditions," Progress in Organic Coatings, vol. 89, pp. 67-74, 2015.

[164] F. Weichelt, R. Emmler, R. Flyunt et al., "ZnO-based UV nanocomposites for wood coatings in outdoor applications," Macromolecular Materials and Engineering, vol. 295, no. 2, pp. 130-136, 2010.

[165] X. Liu, X. Wu, H. Cao et al., "Growth mechanism and properties of ZnO nanorods synthesized by plasma-enhanced chemical vapor deposition," Journal of Applied Physics, vol. 95, no. 6, pp. 3141-3147, 2004.

[166] A. Irzh, I. Genish, L. Klein et al., "Synthesis of $\mathrm{ZnO}$ and $\mathrm{Zn}$ nanoparticles in microwave plasma and their deposition on glass slides," Langmuir, vol. 26, no. 8, pp. 5976-5984, 2010.

[167] K. P. Ningel, D. Theirich, and J. Engemann, "Characterizing the remote plasma polymerization of octafluorocyclobutane induced by RF-driven hollow-cathode discharge," Surface and Coatings Technology, vol. 98, 1-3, pp. 1142-1147, 1998.

[168] S. Tajima and K. Komvopoulos, "Physicochemical properties and morphology of fluorocarbon films synthesized on crosslinked polyethylene by capacitively coupled octafluorocyclobutane plasma," The Journal of Physical Chemistry C, vol. 111, no. 11, pp. 4358-4367, 2007.

[169] H. Andersson, W. van der Wijngaart, P. Griss et al., "Hydrophobic valves of plasma deposited octafluorocyclobutane in DRIE channels," Sensors and Actuators B:

Chemical, vol. 75, no. 1, pp. 136-141, 2001.

[170] E. van de Walle, "Youth views on sustainability: PMMA, from plexiglass window to a packaging for an implantable glucose sensor," Chemistry International, vol. 37, no. 3, 2015.

[171] J. Janecki, J. Drabik, and M. Wolszczak, "Tribological tests in the system of steelcomposite made of phenol formaldehyde resin and the analysis of the effect of plastic grease in resin on the state of surface layer," Chemik, no. 4, pp. 309-316, 2013.

[172] M.-P. G. Laborie and C. E. Frazier, "13C CP/MAS NMR study of a wood/phenolformaldehyde resin bondline," Journal of Materials Science, vol. 41, no. 18, pp. 60016005, 2006.

[173] "Paints and varnishes - Cross-cut tests," 2013, ISO 2409:2013.

[174] "Plastics - Decorative solid surfacing materials - Part 2: Determination of properties Sheet goods," 2007, ISO 19712-2.

[175] "Optics and photonics - Optical coatings - Part 4: Specific test methods," 2012, ISO 9211-4:2012.

[176] "Paints and varnishes - Pull-off test for adhesion," 2016, ISO 4624:2016. 
[177] M. C. Sunny and C. P. Sharma, "Surface modification of corneal contact lens with phosphoryl choline by glow discharge," Biomaterials, Artificial Cells and Immobilization Biotechnology, vol. 19, no. 3, pp. 599-612, 2009.

[178] R. Wang, C. Zhang, X. Liu et al., "Microsecond pulse driven $\mathrm{Ar} / \mathrm{CF}_{4}$ plasma jet for polymethylmethacrylate surface modification at atmospheric pressure," Applied Surface Science, vol. 328, pp. 509-515, 2015.

[179] A. J. Ward and R. D. Short, "A time-of-flight secondary ion mass spectrometry and Xray photoelectron spectroscopy investigation of the structure of plasma polymers prepared from the methacrylate series of monomers," Polymer, vol. 34, no. 20, pp. 4179-4185, 1993.

[180] N. de Geyter, R. Morent, S. van Vlierberghe et al., "Deposition of polymethyl methacrylate on polypropylene substrates using an atmospheric pressure dielectric barrier discharge," Progress in Organic Coatings, vol. 64, no. 2, pp. 230-237, 2009.

[181] G. Avramidis, Surface analytics of atmospheric pressure plasma treated wood and wood-based materials, Dissertation, University of Göttingen, 2014.

[182] K. G. Donohoe and T. Wydeven, "Plasma polymerization of ethylene in an atmospheric pressure-pulsed discharge," Journal of Applied Polymer Science, vol. 23, no. 9, pp. 2591-2601, 1979.

[183] R. Rerich, Abscheidung und Analyse wasserabweisender Schichten auf Holz mittels Plasmapolymerisation von Ethylen, Bachelor thesis, University of Applied Sciences and Arts Hildesheim/Holzminden/Göttingen, 2014.

[184] H. K. Yasuda, "Some important aspects of plasma polymerization," Plasma Processes and Polymers, vol. 2, no. 4, pp. 293-304, 2005.

[185] F. F. Shi, "Recent advances in polymer thin films prepared by plasma polymerization: Synthesis, structural characterization, properties and applications," Surface and Coatings Technology, vol. 82, 1-2, pp. 1-15, 1996.

[186] R. M. Nussbaum, "Natural surface inactivation of scots pine and norway spruce evaluated by contact angle measurements," European Journal of Wood and Wood Products, vol. 57, no. 6, pp. 419-424, 1999.

[187] "Optics and optical instruments - Microscopes - Slides - Part 1: Dimensions, optical properties and marking," 1986, ISO 8037 - 1.

[188] S. Krimm, C. Y. Liang, and G. B. B. M. Sutherland, "Infrared spectra of high polymers. II. Polyethylene," The Journal of Chemical Physics, vol. 25, no. 3, pp. 549$562,1956$. 


\section{Publications}




\title{
Paper I
}

\section{Topographic, Optical and Chemical Properties of Zinc Particle Coatings deposited by means of Atmospheric Pressure Plasma}

\author{
Lena M. Wallenhorst ${ }^{1}$, Leander Loewenthal ${ }^{1}$, Georg Avramidis ${ }^{1}$, Christoph \\ Gerhard $^{1,2}$, Holger Militz ${ }^{3}$, Gisela Ohms ${ }^{1}$, Wolfgang Viöl ${ }^{1,2}$ \\ ${ }^{1}$ University of Applied Sciences and Arts, Laboratory of Laser and Plasma Technologies, \\ Von-Ossietzky-Straße 99, 37085 Göttingen, Germany \\ ${ }^{2}$ Fraunhofer Institute for Surface Engineering and Thin Films, Application Center for Plasma and \\ Photonics, Von-Ossietzky-Straße 100, 37085 Göttingen, Germany \\ ${ }^{3}$ Wood Biology and Wood Products, Burckhardt Institute, Georg-August-University Göttingen, \\ Büsgenweg 4, 37077 Göttingen, Germany
}

\begin{abstract}
In this research, topographic, optical and chemical properties of zinc oxide layers deposited by a cold plasma-spray process were measured. Here, zinc micro particles were fed to the afterglow of a plasma spark discharge whereas the substrates were placed in a quite cold zone of the effluent plasma jet. In this vein, almost closed layers were realised on different samples. As ascertained by laser scanning and atomic force microscopic measurements the particle size of the basic layer is in the nanometre scale. Additionally, larger particles and agglomerates were found on its top. The results indicate a partial plasma-induced diminishment of the initial particles, most probably due to melting or vaporisation. Itis further shown that the plasma gives rise to an increased oxidation of such particles as confirmed by X-ray photoelectron spectroscopy. Quantitative analysis of the resulting mixed layer was performed. It is shown that the deposited layers consist of zinc oxide and elemental zinc in approximately equal shares. In addition, the layer's band gap energy was determined by spectroscopic analysis. Here, considerable UV-blocking properties of the deposited layers were observed. Possible underlying effects as well as potential applications are presented.
\end{abstract}

Keywords: Plasma, Zinc Oxide, Particle Deposition, Protective Coatings, XPS

\section{Introduction}

Zinc oxide features a number of properties that permit manifold applications. Its use as a corrosion protection even extends to challenging environments such as sea water [1]. Since it is a semiconductor with a rather wide band gap energy of 3.2-3.4 eV [2-4], it is also of interest in the development of solar cells [5].This band gap energy corresponds to light in the near UV range (approx. 365-387 nm), resulting in a high absorption rate of light of shorter wavelengths. This property makes $\mathrm{ZnO}$ suitable for protecting surfaces from photo-degradation in outdoor use [6,7]. Moreover, $\mathrm{ZnO}$ is well known for its biocidal properties, thus ensuring protection against bacteria and fungi $[8,9]$. All these protective properties are desirable in wood protection. Contrarily to many naturally resistant tropical woods, European and Northern American local wood species, for example spruce or beech, are especially subject to biotic and abiotic degradation processes such as photodegradation and damage due to fungi attack when used outdoors. Therefore, local wood has to be protected and the development of new coatings is a particularly promising approach to achieve this goal. However, the creation of highly efficient protective systems that feature a low toxicity remains challenging. On the other hand, many advanced coatings that provide protection to glass or plastics have to be applied under vacuum conditions. This is problematic when considering the large dimensions of common wood products. Other techniques require high temperatures that may destroy thermosensitive materials such as wood. 
Emerging techniques for surface modifications, and particularly for the deposition of coatings, are plasma processes at atmospheric pressure. However, due to its thermosensitive nature, wood can-not be treated by all kinds of plasmas that are typically applied for surface treatments since some plasma discharges feature high gas temperatures. Against this background, low-temperature plasma polymerisation [10-16] represents an appropriate method for reducing the water uptake in wood by applying hydrophobic thin films to the wood surface. For this purpose, in particular dielectric barrier discharges which stand out due to a low gas temperature in the range of some tens of centigrades are applied to deposit coatings based on a gaseous or liquid precursor.

Another promising approach is the deposition of solid materials in powder form, requiring different plasma setups. The method described in this and previous articles [17-19] is conceivable to eventually yield an easy deposition process suitable for in-line procession. By applying this technique, it should be possible to minimise or even relinquish the use of binding agents.

In this study the properties of zinc powder-based coatings deposited in a plasma-spray coating process were investigated. This plasma coating process is also suitable for treating thermosensitive materials, but limits the range of materials that can be deposited since a relatively low melting point of the used powder material is required. Hence, elemental zinc $(\mathrm{Zn})$ was used as a starting material instead of zinc oxide $(\mathrm{ZnO})$. Coatings were deposited on fused silica and acrylic glass substrates with low surface roughness. Subsequently, LSM and AFM measurements were performed in order to investigate the topography of the coating's surface and to estimate the approximate sizes or heights of the particles and particle agglomerations. Here, the heterogeneously structured surface does not permit a thorough determination of a film's thickness. Since the resulting coatings are intended for an application as wood preservative, the absorption behaviour of the thin coatings in the UV range was studied via UV/VIS spectrometry. Moreover, the chemical structure was investigated directly on the coating's pristine surface and on etched surfaces.

\section{Methods}

\section{Deposition process}

Zinc particles $\left(\mathrm{d}_{50}=13 \mu \mathrm{m}\right.$, Standart Zinkflake GTT, ECKART GmbH) were applied to different substrates using a setup for plasma-spray coating processes as described in [17-19]. A sketch of the assembly is shown in Figure 1.

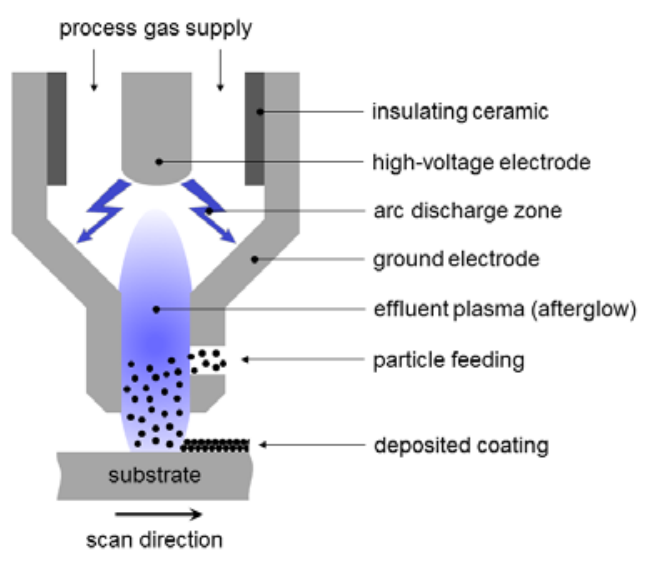

Figure 1: Assembly to deposit coatings using a plasma-assisted deposition process.

Here, the samples are placed on a linear stage in order to realise homogeneous large-scale coatings by scanning the sample at appropriate scan speeds. Further, the setup includes a commercially available power supply generating a pulsed high voltage and a plasma source based on a standard plasma jet (Plasmabrush from Reinhausen Plasma $\mathrm{GmbH}$ ) with an optimised spraying nozzle. In this assembly, the process gas is ionised by an arc discharge and driven out to form a plasma jet whereas the particle aerosol is added to the afterglow of the discharge. To generate the particle aerosol, a uniformly compressed cylindrical powder column of dry Zn powder was moved towards a rotating brush. The 
feed rate finally results from the velocity of the powder column. The particles were then dispersed in the carrier gas (compressed air) which streamed above the brush and transported towards the spraying nozzle. The plasma is generated by applying an ignition voltage of approx. $15 \mathrm{kV}$, whereas the effective voltage amounts to $2-3 \mathrm{kV}$. The input power reaches a maximum of $2 \mathrm{~kW}$ where high voltage pulses with a pulse duration of 5-10 $\mu$ s and a pulse repetition rate of $50 \mathrm{kHz}$ are applied [17-19]. Even though arc discharges typically feature high gas temperatures (several thousand centigrades), the substrate's temperature can be kept comparatively low (below $100^{\circ} \mathrm{C}$ at a working distance of $>20 \mathrm{~mm}$ [18]) by means of a low electrical current, a transient discharge and an appropriate working distance. This permits coating of thermosensitive materials such as wood. The feasibility of this setup for coating beech (Fagus sylvatica L.) and pine wood (Pinus sylvestris L.) has already been shown [17-19]. On the other hand, it restricts the choice of processible particles to those featuring a low melting point. $\mathrm{ZnO}$ is of particular interest in manifold applications, but due to its high melting/sublimation point, it cannot be directly deposited in the described way. Hence, elemental zinc was used, with the expectation of a high oxidation rate during and after the deposition process, consequently resulting in the formation of zinc oxide.

For all deposited coatings, compressed air was used as both process gas and carrier gas to form the aerosol along with the zinc particles. The process gas flow, working distance and carrier gas pressure were kept constant at $30 \mathrm{l} / \mathrm{min}, 16 \mathrm{~mm}$ and 1.6 bar, respectively. Table 1 summarises the various deposition parameters. Sample X denotes the sample prepared for XPS measurements (fused silica), whereas samples $A_{i}, B_{i}$, and $C_{i}$ were used for UV/VIS analysis and topographic measurements (acrylic glass). For the acrylic glass (Plexiglas GS 2458 from Evonik Performance Materials GmbH), a glass transition temperature of $(118.5 \pm 0.6)^{\circ} \mathrm{C}$ was measured according to [20] by Differential Scanning Calorimetry (DSC 3+, Mettler-Toledo $\mathrm{GmbH}$ ). The Vicat softening temperature equals $100^{\circ} \mathrm{C}$ as indicated by the manufacturer.

Table 1: Coating parameter sets and substrate materials for plasma-spray deposition of zinc particles.

\begin{tabular}{lccc}
\hline Sample no. & Substrate material & $\begin{array}{c}\text { Powder feed rate } \\
{\left[\mathbf{c m}^{3} / \mathbf{h}\right]}\end{array}$ & $\begin{array}{c}\text { Substrate scan speed } \\
{[\mathbf{m m} / \mathbf{s}]}\end{array}$ \\
\hline $\mathrm{X}$ & Fused silica & 5.6 & 40 \\
$\mathrm{~A}_{1}, \mathrm{~A}_{2}$ & Acrylic glass & 3.2 & 40 \\
$\mathrm{~B}_{1}, \mathrm{~B}_{2}$ & Acrylic glass & 6 & 40 \\
$\mathrm{C}_{1}, \mathrm{C}_{2}$ & Acrylic glass & 6 & 50 \\
\hline
\end{tabular}

\section{Surface analytical methods}

The coating's topography was examined by Confocal Laser Scanning Microscopy (using a LSM 700 from Zeiss) and Atomic Force Microscopy (AFM, using a NeaSnom from NeaSpec). For a larger overview, LSM measurements were performed using a laser with a wavelength of $405 \mathrm{~nm}$ and an objective lens magnification of $\mathrm{M}=20 \mathrm{x}$ (numerical aperture $\mathrm{NA}=0.6$ ). Evaluation of the measurements was carried out by use of the software ZEN 2012 Black [21]. AFM scans were executed with silicon probes (Arrow-NCPt from NanoWorld) with a tip radius of approx. $10 \mathrm{~nm}$ at a resonant frequency of approx. $280 \mathrm{kHz}$. All measurements were performed in ambient atmosphere and tapping mode. The scan rate was approx. $0.14 \mathrm{~Hz}$ and the set-point ratio was approx. 0.7. To ensure reliability, images were acquired on five different areas of each sample's surface. The evaluation software Gwyddion 2.44 from the Czech Metrology Institute was used for data processing [22].

Optical measurements were carried out at room temperature using a UV/VIS spectrophotometer (Lambda 650 from Perkin Elmer) to obtain transmittance spectra for wavelengths ranging from 290 to $550 \mathrm{~nm}$. The lower limit of $290 \mathrm{~nm}$ was chosen since sunlight of shorter wavelengths is absorbed by ozone in the atmosphere [23]. For this reason, Zn-coatings were deposited on acrylic glass that exhibits a high optical transmittance also in the UV-range.

The coating's chemical structure was studied by X-ray photo-electron spectroscopy (XPS) by means of a PHI 5000 Versa ProbeII system from ULVAC-PHI. A monochromatic Al $\mathrm{K}_{\alpha}$ source with a photon energy of $1486.6 \mathrm{eV}$ was used to irradiate a spot of $200 \mu \mathrm{m}$ in diameter on the surface. The detector's minimal resolution amounted to $0.45 \mathrm{eV}$ for the $\mathrm{Ag} 3 \mathrm{~d}_{5 / 2}$ peak. Detail spectra were acquired 
at a pass energy of $23.5 \mathrm{eV}$, survey spectra at $187.85 \mathrm{eV}$ and depth profiles at $46.95 \mathrm{eV}$. Depth profiles were obtained by etching the surface with argon ions at an accelerating voltage of $1 \mathrm{kV}$ and a sample current of $1 \mu \mathrm{A}$. The size of the etched surface amounted to $3 \times 3 \mathrm{~mm}^{2}$. In all measurements, charging effects were reduced by the system's neutralisation system consisting of low energy argon ions and electrons. As reference samples, Zinc foil (99.98\% purity from Alfa Aesar) and zinc oxide $(99.9 \%$ purity from Sigma Aldrich) were used to determine the relative peak positions. Since the zinc coatings were deposited on silica glass, the reference samples were also placed on the same insulating substrate. Prior to the measurement of a detail spectrum on the zinc foil, it was sputter cleaned to ensure a complete absence of oxygen.

All intensity spectra were analysed using the software MultiPak from PHI [24] and were displayed against binding energies with respect to the Fermi level. For spectral deconvolution and quantitative analysis, a Shirley-type background was subtracted and a symmetric Gauss-Lorentz profile was fitted to all peaks except for the elemental contribution to the $\mathrm{Zn} 2 \mathrm{p}_{3 / 2}$ peak; this was instead fitted with an asymmetric Gauss-Lorentz function. An overall error of $5 \%$ was assumed for all stoichiometric results, accounting both for the measurement and fitting error. The spectra of the deposited coatings were adjusted to give a binding energy (BE) of $103.3 \mathrm{eV}$ for the $\mathrm{Si} 2 \mathrm{p}$ peak of $\mathrm{SiO}_{2}$. Subsequently, the resulting positions of the Zinc $\mathrm{L}_{3} \mathrm{M}_{45} \mathrm{M}_{45}$ peak (hereafter referred to as LMM) of the etched coating were used to shift the reference spectra, since the reference samples appeared to be composed only of elemental zinc and $\mathrm{ZnO}$ (and some carbon in the case of $\mathrm{ZnO}$ powder). Moreover, an investigation of Auger peaks as presented below in Table 3 assures a reliable identification of species since they do not suffer from an overlap as strong as the $\mathrm{Zn} 2 \mathrm{p}_{3 / 2}$ peak for elemental and oxidised $\mathrm{Zn}$ and can therefore support the stoichiometric results in a qualitative way.

\section{Results and Discussion}

\section{Topography}

Figure 2 shows the height structure as measured by LSM for a representative measurement of sample $\mathrm{B}_{1}$ (coated acrylic glass, scanned area: $320 \times 320 \mu \mathrm{m}^{2}$ ). For a better visualisation, the grey values are gamma-corrected by a value of $\gamma=0.45$. Six measurements in total were executed on each sample; all images were tilted by applying a plane fit and slightly smoothed by a Gaussian low pass filter with a kernel size of 5 pixels. Qualitatively, the shape of the larger particles resembles the flake form of the original powder. Since the deposited coatings are rather inhomogeneous on a microscopic scale, a reliable quantitative analysis of height profiles cannot be performed. Nevertheless, the maximum peak-to-valley value $\mathrm{WSt}=(10.8 \pm 1.2) \mu \mathrm{m}$ was determined to give an idea about the height. Due to reflection losses close to large particles (cf. Figure 2(b)), a threshold was defined to minimise distortion by these valleys.

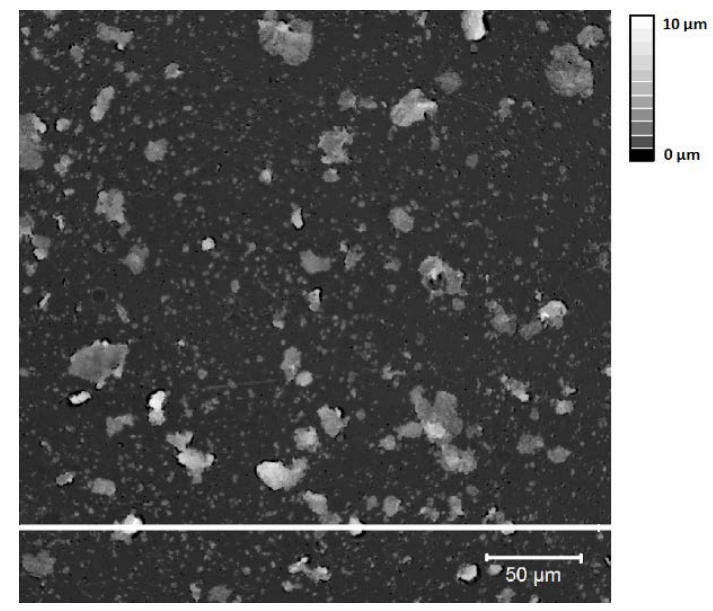

(a)

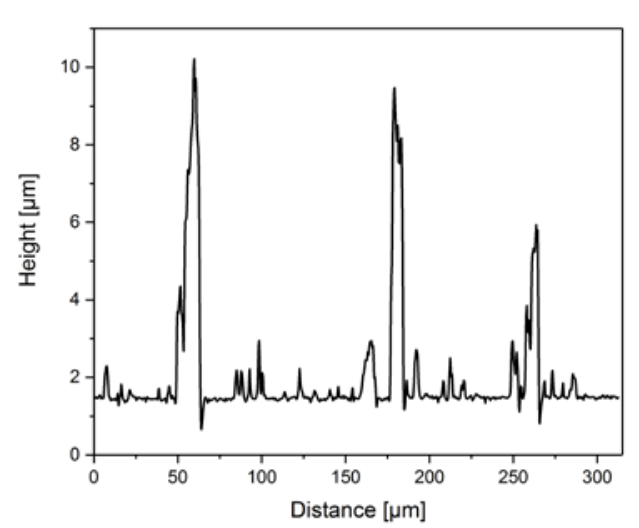

(b)

Figure 2: Topography as measured by laser scanning microscopy (scanned area: $320 \times 320 \mathrm{\mu m}^{2}$ ). (a) shows the height structure of sample $B_{1}$. (b) depicts an exemplary height profile along the white line in (a), before setting a threshold.

In addition, AFM measurements (scanned area: $3 \times 3 \mu \mathrm{m}^{2}$ ) were performed in order to investigate the space between larger particles/agglomerations. The raw data were corrected by a polynomial row 
alignment and by the application of a sharpening filter which includes Gaussian smoothing and a subsequent extrapolation backwards to estimate a sharper image (kernel size: 3 pixels). Figure 3 shows a representative example for the topography of coated acrylic glass. The measurements revealed a roughly structured surface composed of nanoparticles. The particles' shape differs notably from the form of the original powder, which could be due to an evaporation of material in the plasma and a recondensation on the substrate surface. Analogous to the evaluation of the LSM measurements, the maximum peak-to-valley value (here referred to as $\mathrm{PtV}$ ) was determined for five areas on the sample, yielding a value of $\mathrm{PtV}=(154 \pm 15) \mathrm{nm}$.

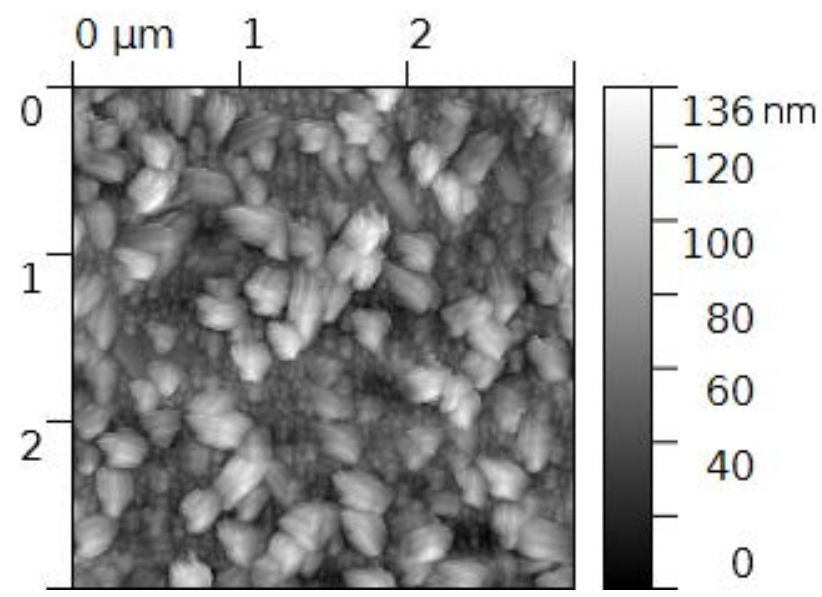

Figure 3: Topography of coated acrylic glass (sample $B_{1}$ ) as measured by AFM.

\section{Optical properties}

Optical properties of Zn-powder deposited onto acrylic glass were studied by investigating transmittance spectra of samples $A_{i}, B_{i}$ and $C_{i}$. To exclude changes in transmittance $T$ of the substrate only by virtue of heating during the plasma coating process, the reference samples were also plasma treated using the same plasma parameters but without the addition of any powder. All spectra were slightly smoothed by applying a Savitzky-Golay filter (2nd degree polynomial). Figure 4(a) depicts a representative transmittance spectrum (sample $\mathrm{B}_{1}$ ) along with the corresponding reference spectrum and Figure 4(b) shows this spectrum of sampleB1after normalisation to the spectrum of the uncoated reference sample. It shows a transmittance of $81-82 \%$ in the range of approx. $371-550 \mathrm{~nm}$, whereas a sharp transmittance edge and subsequently a strong decrease in transmittance for shorter wave-lengths are clearly visible.

For direct bandgap transitions, the absorption process is described by the following relation [25]:

$$
\alpha \cdot h v=A \cdot\left(h v-E_{B G}\right)^{1 / 2}=: g(h v)
$$

where $\alpha$ is the absorption coefficient, $\mathrm{E}_{\mathrm{BG}}$ the bandgap energy, $h v$ the photon energy and $\mathrm{A}$ a characteristic coefficient. This equation holds for photon energies slightly larger than $\mathrm{E}_{\mathrm{BG}}$. Since $\alpha \sim-\ln (\mathrm{T})$, the function $f(h v):=(-\ln (\mathrm{T}) h v)^{2}$ was investigated (Figure 4(c)). The bandgap energy can be determined by extrapolating the linear part of $g(h v)^{2}$ or $f(h v)$ and calculating the limit as $\alpha \rightarrow 0$.

Table 2 summarises the results for each set of parameters and the overall average. The slight variations of coating parameters do not appear to have a crucial impact on the band gap energy.

Table 2: Band gap energies for 3 different coating conditions.

\begin{tabular}{lc}
\hline Sample no. & $\mathbf{E}_{\mathbf{B G}}[\mathbf{e V}]$ \\
\hline $\mathrm{A}_{1}, \mathrm{~A}_{2}$ & $3.20 \pm 0.02$ \\
$\mathrm{~B}_{1}, \mathrm{~B}_{2}$ & $3.21 \pm 0.01$ \\
$\mathrm{C}_{1}, \mathrm{C}_{2}$ & $3.19 \pm 0.02$ \\
Average & $3.20 \pm 0.02$ \\
\hline
\end{tabular}


A bandgap energy for $\mathrm{ZnO}$ of $\mathrm{E}_{\mathrm{BG}}=3.20 \mathrm{eV}$ or even lower can be found in literature [2,26], however, most publications report higher values. Srikant and Clarke [27] studied the influence of different substrates on the bandgap energy of $\mathrm{ZnO}$ thin films and found a significantly lower value for coated fused silica than for C- and R-plane sapphire. Contrarily to the coatings on sapphire, the investigated coating on fused silica exhibited a roughly structured surface composed of nanoparticles. The authors concluded that due to the small grain size, the potential varies inside the particles by virtue of boundary effects instead of corresponding to flat bands. Hence, the energy needed for a band transition varies, leading to a lower effective bandgap energy. Since the $\mathrm{ZnO}$ coatings in the present study exhibit a similar structure as ascertained by AFM, we assume the same effects to take place.

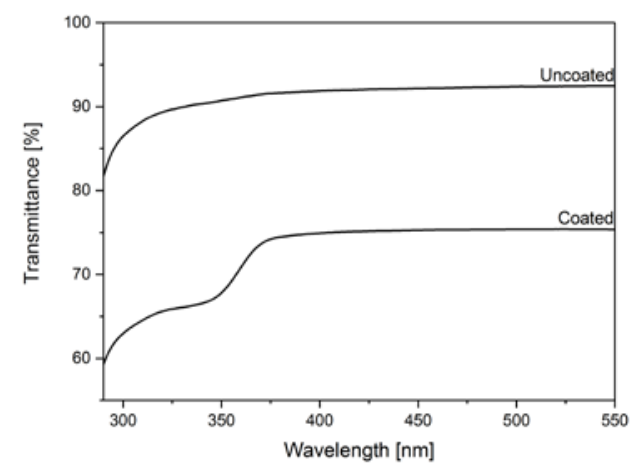

(a)

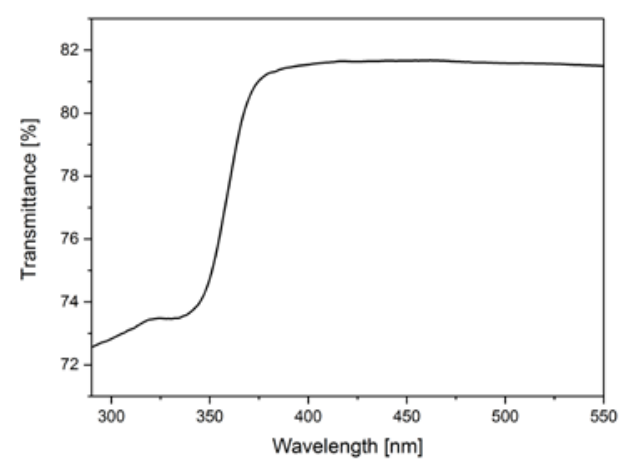

(b)

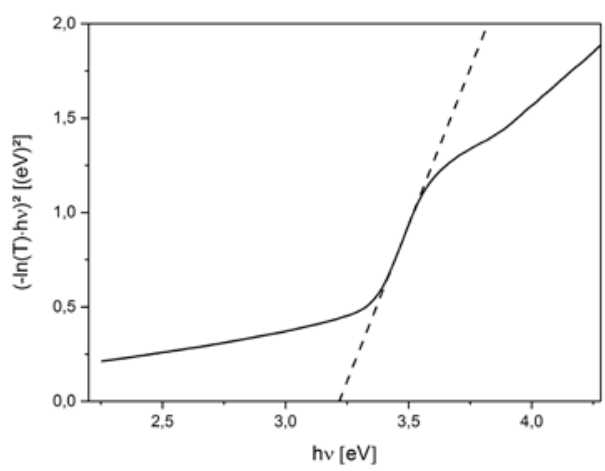

(c)

Figure 4: (a) Comparis on of the air plasma treated and the coated samples. (b) Normalised transmittance spectrum of coated acrylic glass showing an enhanced absorption in the UV-range. (c) Determination of the bandgap energy by extrapolating $f(h v) \sim \alpha h v^{2}$.

\section{Chemical structure}

Table 3 summarises results from XPS measurements of the reference samples, i. e. sputter cleaned elemental zinc foil and pure $\mathrm{ZnO}$ powder. It lists the positions of the $\mathrm{Zn} 2 \mathrm{p}_{3 / 2}$ peak after shifting the main peak of the LMM to $493.39 \mathrm{eV}$ and $497.78 \mathrm{eV}$ for $\mathrm{Zn}$ and $\mathrm{ZnO}$, respectively. As explained Section 2, these positions of the LMM main peaks were taken from the spectrum displayed in Figure 9(b) of the etched $\mathrm{Zn} / \mathrm{ZnO}$ coating with charge referencing of the Si2p peak at $103.3 \mathrm{eV}$ for $\mathrm{SiO}_{2}$ [28]. Furthermore, the resulting Modified Auger Parameters (MAP) and the position of the O1s peak for metal oxide are included.

Table 3: Zn2p $\mathrm{p}_{3 / 2}$, O1s, LMM and MAP values for pure $\mathrm{Zn}$ and $\mathrm{ZnO}$ for spectra acquired at pass energies of $23.5 \mathrm{eV}$ $(\mathrm{Zn})$ and $46.95 \mathrm{eV}(\mathrm{ZnO})$. * denotes shifted values, see text for explanation.

\begin{tabular}{lccccc}
\hline Compound & O1s [eV] & Zn2p $_{\mathbf{3} / \mathbf{2}}[\mathbf{e V}]$ & & LMM [eV] & MAP [eV] \\
\hline $\mathrm{ZnO}$ & 529.87 & 1021.12 & Peak 1 & $497.78^{*}$ & 2009.94 \\
& & & Peak 2 & 494.41 & 2013.31 \\
$\mathrm{Zn}$ & \multirow{2}{*}{1020.55} & Peak 1 & $493.39^{*}$ & 2013.76 \\
& & & Peak 2 & 490.10 & 2017.50 \\
\hline
\end{tabular}


These results are in good agreement with literature MAP values [29] and with values for the relative LMM positions [30]. The difference between the positions for $\mathrm{Zn} 2 \mathrm{p}_{3 / 2}$ (oxygen bound) and $\mathrm{O} 1 \mathrm{~s}$ ( $\mathrm{Zn}$ bound) also concurs well with numerous references [3,31-33]. Figure 5 shows a survey spectrum of the pristine plasma-deposited coating. Except for a negligible amount of nitrogen, it consists only of zinc, oxygen and carbon. A small amount of silicon manifests via the peak at $103.3 \mathrm{eV}$, but due to its overlap with a shake-up from $\mathrm{Zn}$, it is barely visible in this spectrum.

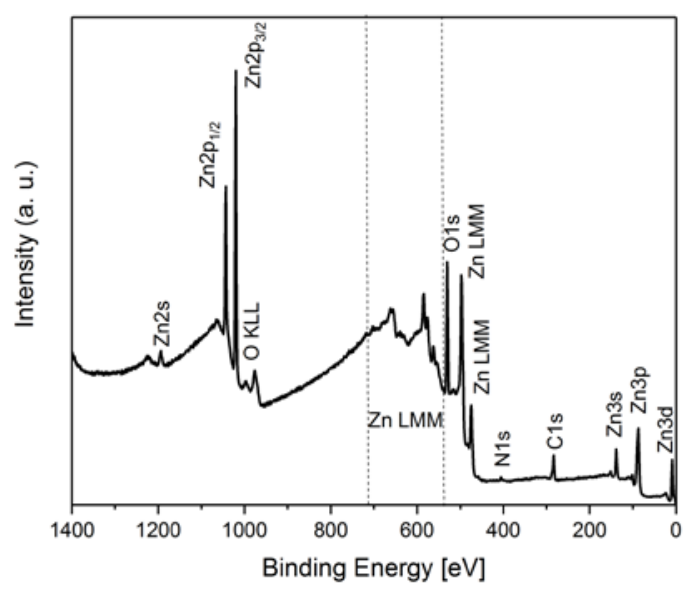

Figure 5: Survey spectrum of the pristine coating at a pass energy of $187.85 \mathrm{eV}$.

For the plasma-deposited coating, a depth profile was acquired to examine the evolution of the relative amounts of zinc, oxygen, silicon and carbon. At first, spectra were acquired after etching intervals of $0.5 \mathrm{~min}$ for in total $10 \mathrm{~min}$ of etching. Subsequently, two spectra were measured each after 3 more minutes of etching. Even though the argon bombardment can lead to preferential sputtering and therefore a reduction of oxides [34-36], it does not seem to occur for $\mathrm{ZnO}[37,38]$. Therefore, any effects on the stoichiometry were neglected. An overview of the chemical composition's variation with depth is depicted in Figure 6.

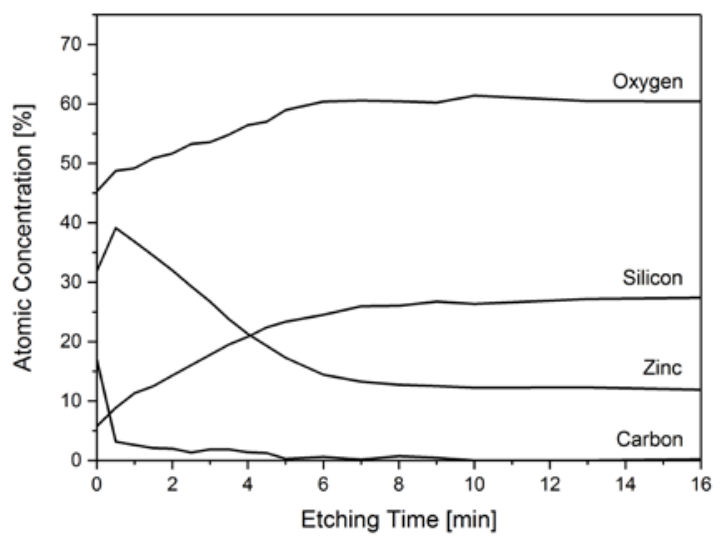

Figure 6: Variation of the chemical composition with etching time and depth.

The trend of the $\mathrm{Zn}$ content with increasing etching time corresponds to the expectations formed from the AFM pictures: Some large (accumulated) particles are deposited on the surface and an almost closed layer of smaller particles is situated in the spaces in between. There are two possible explanations for this observation. One possibility is that the original $\mathrm{Zn}$ particles are not only molten but also evaporated within the plasma to form new and very small particles when condensing on the substrate. The other possibility involves a selection process in which only the very small particles comprised in the powder adhere well to the substrate's surface whereas other, larger particles are mostly blown off the sample by virtue of the process gas flow. This thin layer is completely removed 
after approximately $5 \mathrm{~min}$ of etching. Since the coating is roughly structured and consists of different $\mathrm{Zn}$ compounds $\left(\mathrm{ZnO}\right.$, elemental $\mathrm{Zn}$ and $\mathrm{Zn}(\mathrm{OH})_{2}$, cf. Table 4), a definite thickness cannot be calculated from the data. Nevertheless, it can be approximated by the etching rate of $\mathrm{SiO}_{2}$ for the applied set-tings $(1.75 \mathrm{~nm} / \mathrm{min})$, leading to an approximate film thickness of $8.8 \mathrm{~nm}$. After the first etching interval $(0.5 \mathrm{~min})$, most of the adventitious carbon is already removed. Since neither the powder nor the cleaned substrate was kept under vacuum/inert conditions, accumulations of carbon could have been expected at the interface between the thin coating and the glass or also in general within the coating, since every original particle was supposedly surrounded by a thin carbon coating. Given that this was not observed, it suggests a cleaning process parallel to the deposition process which might be due to the photocatalytic activity of $\mathrm{ZnO}$ [39-42] induced by UV light that originates from the plasma $[43,44]$. Consequently, only a carbon layer on top of the coating is formed after the deposition process.

Figure 7(a) Figure 7(b) shows the evolution of the $\mathrm{O} 1 \mathrm{~s}$ and $\mathrm{Zn} 2 \mathrm{p}_{3 / 2}$ peaks, respectively, during etching. For clarity, only a few spectra are depicted. Figure 7(a) illustrates a distinct shift of the $\mathrm{Zn} 2 \mathrm{p}_{3 / 2}$ towards lower binding energies which indicates a change in chemical bonding states. Figure 7(b) reveals the gradual exposure of $\mathrm{SiO}_{2}$ by the appearance of a strong O1s peak at $532.6 \mathrm{eV}$.

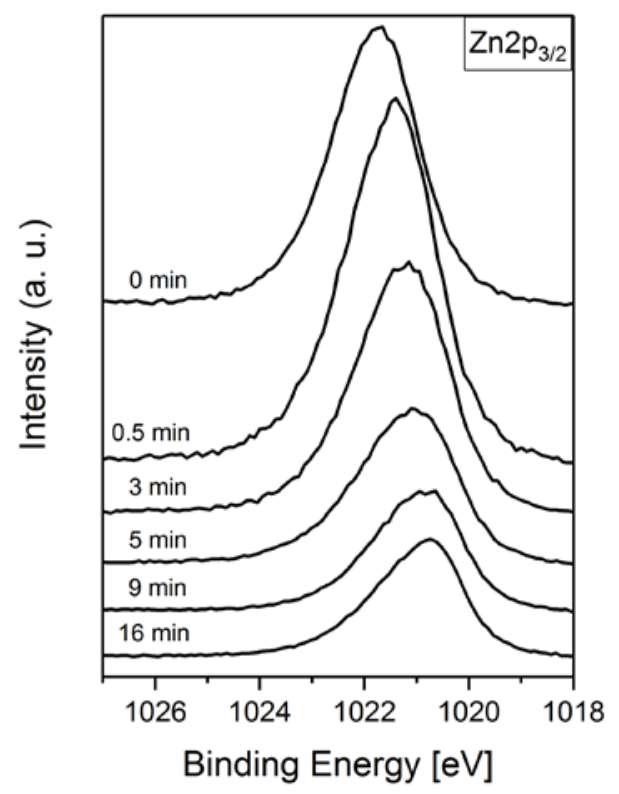

(a)

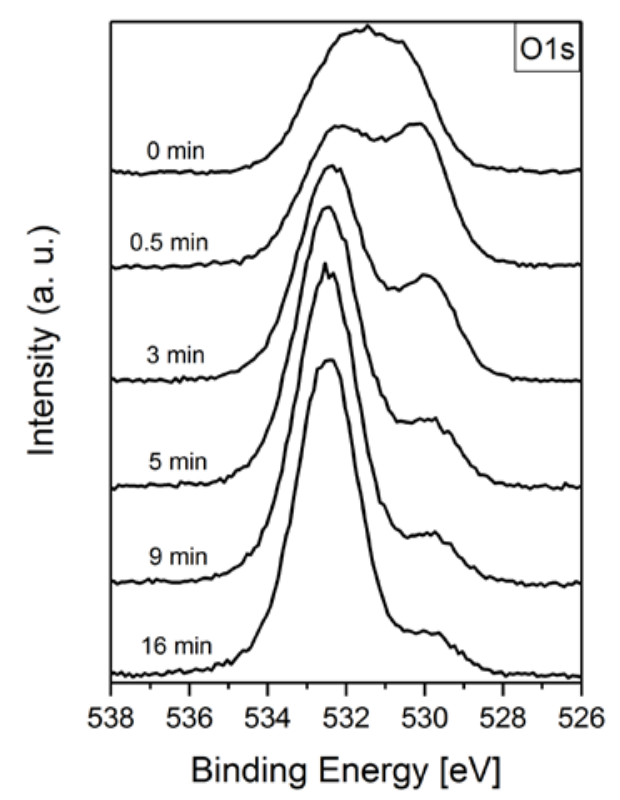

(b)

Figure 7: Selected spectra of the profile after $0,0.5,3,5,9$ and 16 min of etching. (a) Changes in the peak position of the $\mathrm{Zn} 2 \mathrm{p}_{3 / 2}$. Since these peaks result from mixed systems consisting of elemental $\mathrm{Zn}, \mathrm{ZnO}$ and $\mathrm{Zn}(\mathrm{OH})_{2}$, the peak positions differ from those of pure $\mathrm{Zn}$ and $\mathrm{ZnO}$ as presented in Table 3. (b) Variation of the O1s peak during the removal of metallic bound oxygen and exposure of oxygen bound to $\mathrm{Si}$.

Further investigation of the chemical composition was performed by acquiring detail spectra of the pristine coating and the surface that had been etched for a total of $16 \mathrm{~min}$. For spectral deconvolution, the O1s peak was divided a priori into three different contributions: oxygen from $\mathrm{ZnO}$, from $\mathrm{SiO}_{2}$ and from $\mathrm{Zn}(\mathrm{OH})_{2}$ or carbonic bounds (cf. Figure 8). The decomposition of the $\mathrm{Zn} 2 \mathrm{p}_{3 / 2}$ peak into its contributions from $\mathrm{ZnO}, \mathrm{Zn}$ and $\mathrm{Zn}(\mathrm{OH})_{2}$ is shown in Figure 9(a). Investigation of the LMM peaks (Figure 9(b)) yields a sound identification of $\mathrm{Zn}$ species since the two peaks resulting from elemental $\mathrm{Zn}$ (etched surface) are clearly separated from those generated by oxidised zinc.

To qualitatively distinguish the oxidation induced by the deposition process from the native oxide layer, Figure 10 compares the LMM peaks from the pristine coating to those from untreated $\mathrm{Zn}$ powder. It shows that contrarily to the spectrum of the plasma coating, the LMM peaks of the untreated powder feature clearly visible contributions resulting from elemental $\mathrm{Zn}$ (peak 3 and 4). 
Chen et al. [32] examined the growth rate of the native oxide layer on $\mathrm{Zn}$ for $72 \mathrm{~h}$ and found that, after an initial phase of about $10 \mathrm{~h}$, the growth rate slows down and exhibits a linear behaviour with a growth rate of approx. $0.01 \mathrm{~nm} \mathrm{~h}^{-1}$. Therefore, the oxidative component of the plasma coating is assumed to result mainly from oxidation induced by the deposition process.

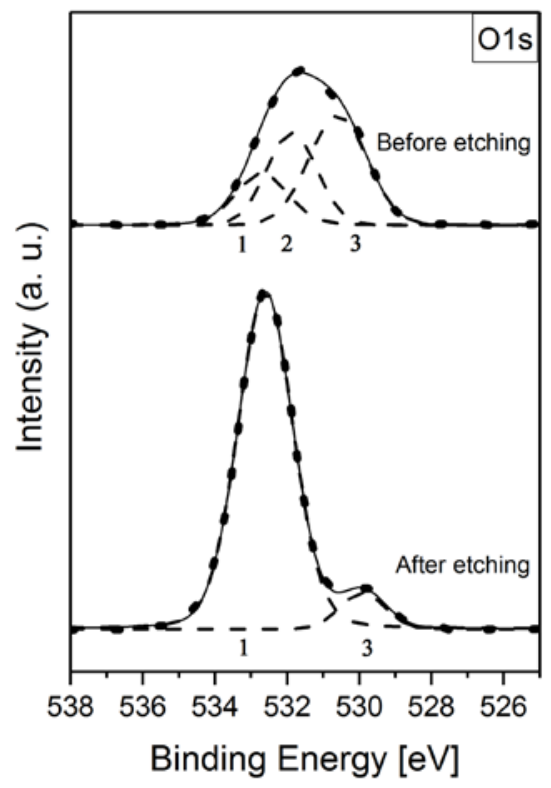

Figure 8: Decomposition of the $\mathrm{O} 1 \mathrm{~s}$ peak into its contributions from $\mathrm{SiO}_{2}$ (peak 1), $\mathrm{Zn}(\mathrm{OH})_{2} / \mathrm{carbon}$ compounds (peak 2) and ZnO (peak 3).

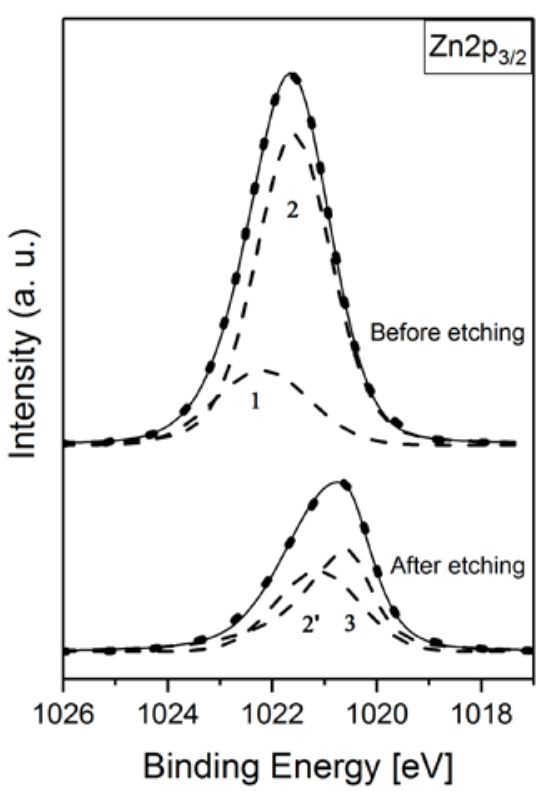

(a)

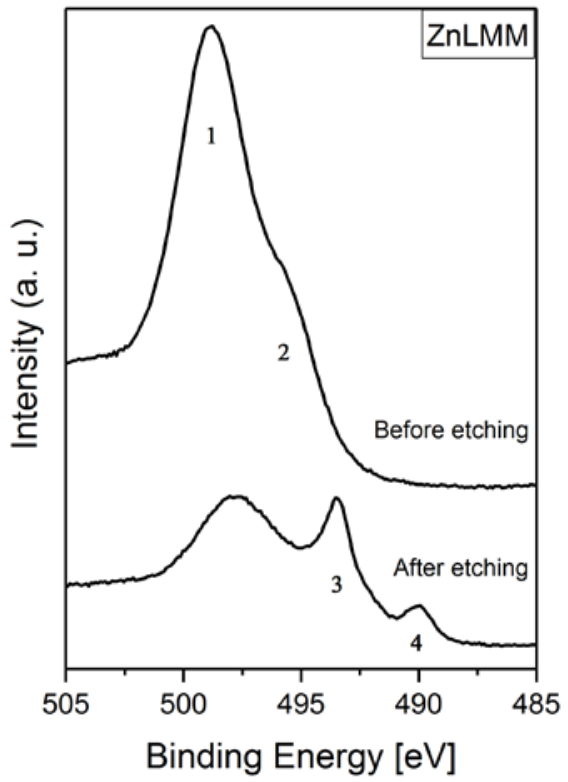

(b)

Figure 9: (a) Decomposition of the $\mathrm{Zn2p3/2peak} \mathrm{into} \mathrm{contributions} \mathrm{from} \mathrm{elemental} \mathrm{Zn}, \mathrm{ZnO}$ and $\mathrm{Zn}(\mathrm{OH})_{2}$ before and after etching. The dotted lines depict the raw data; dashed lines indicate the fit curves with peaks for $\mathrm{Zn}(\mathrm{OH})_{2}$ at $1022.19 \mathrm{eV}$ (peak 1) and $\mathrm{ZnO}$ at $1021.59 \mathrm{eV}$ (peak 2) before etching, as well as $\mathrm{ZnO}$ at $1021.13 \mathrm{eV}$ (peak 2_) and elemental $\mathrm{Zn}$ at $1020.56 \mathrm{eV}$ (peak 3 ) after etching. The resulting cumulative fits are displayed as solid lines. (b) LMM before and after etching. Peaks at $497.78 \mathrm{eV}$ (pe ak 1) and $494.58 \mathrm{eV}$ (peak 2) represent the presence of $\mathrm{ZnO}$; peaks at 493.39 eV (peak 3) and $490 \mathrm{eV}$ (peak 4) stand for elemental $\mathrm{Zn}$. In the spectrum for the pristine surface, the LMMs are slightly shifted towards higher binding energies $(498.76 \mathrm{eV}$ and $495.63 \mathrm{eV})$ due to the presence of $\mathrm{Zn}(\mathrm{OH})_{2}$. 


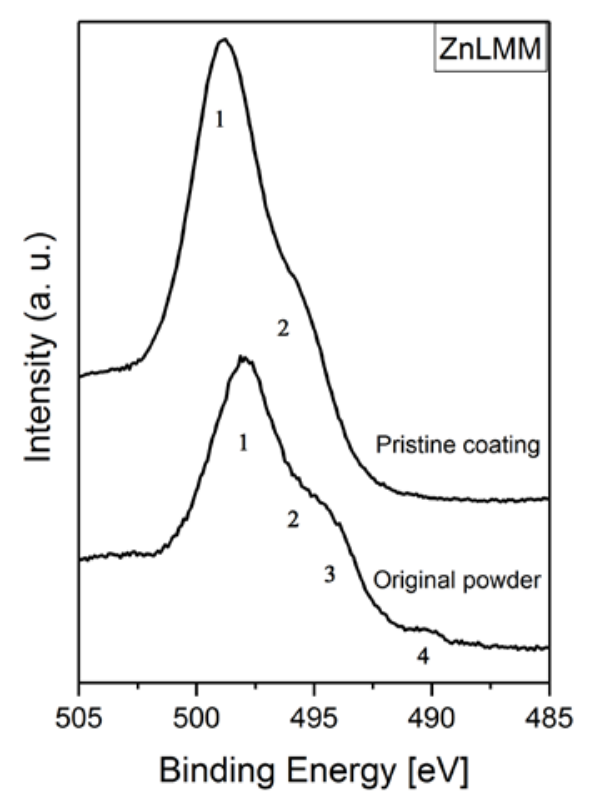

Figure 10: Comparison of the Auger peaks of the pristine coating and untreated powder. While the coating only exhibits peaks corresponding to $\mathrm{ZnO}$ (peak 1 and 2), the powder also clearly features peaks resulting from elemental Zn (peak 3 and 4).

Table 4Table 6 summarise the chemical composition of the surface before and after etching. Since the contribution from $\mathrm{Zn}(\mathrm{OH})_{2}$ to the oxygen peak strongly overlaps with the contribution from carbon bound to oxygen, those contributions were not separated in the decomposition of the O1s peak. Instead, the carbon $\mathrm{C} 1 \mathrm{~s}$ peak was deconvoluted into its contributions from $\mathrm{C}-\mathrm{C} / \mathrm{C}-\mathrm{H}$ (labelled peak no. A), $\mathrm{C}-\mathrm{OH} / \mathrm{C}-\mathrm{O}-\mathrm{C}$ (peak no. $\mathrm{B}$ ), $\mathrm{C}=\mathrm{O}$ (peak no. $\mathrm{C}$ ), $\mathrm{O}-\mathrm{C}=\mathrm{O}$ (peak no. $\mathrm{D}$ ), and carbonates $-\mathrm{CO}_{3}$ (peak no. E) [45]. Table 5 shows that the amount of carbonates appears to be very low, hence, the presence of $\mathrm{ZnCO}_{3}$ was neglected in the course of the analysis. Since the sample was not prepared and stored in an inert atmosphere, the other compounds should result from adventitious carbon. When assuming a ratio of $\mathrm{C}: \mathrm{O}$ of $1: 1$ for peaks no. $\mathrm{B}, \mathrm{C}$, and $\mathrm{D}$ and a ratio of $1: 3$ for peak $\mathrm{E}$ as suggested by [45], the remaining oxygen from the mixed $\mathrm{Zn}(\mathrm{OH})_{2} /$ carbon compounds as derived from the $\mathrm{O} 1 \mathrm{~s}$ peak (12 at. \%) matches twice the amount of $\mathrm{Zn}$ from $\mathrm{Zn}(\mathrm{OH})_{2}$ resulting from its contribution to the $\mathrm{Zn} 2 \mathrm{p}_{3 / 2}$ peak (11.8 at. \%) within the error tolerance. As expected by the shifted $\mathrm{Zn} 2 \mathrm{p}_{3 / 2}$ position to lower binding energies in Figure 7(a), a reduction of $\mathrm{Zn}(\mathrm{OH})_{2}$ and $\mathrm{ZnO}$ for the benefit of elemental $\mathrm{Zn}$ takes place. It shows that $\mathrm{Zn}(\mathrm{OH})_{2}$ is completely removed by etching whereas a large amount of $\mathrm{ZnO}$ is present not only on the surface, but also within the bulk material. Thus, the plasma coating procedure induces oxidation processes. Since the coatings partly exhibit nanostructures, the increased reactivity also supports these oxidation effects.

Table 4: Pristine surface: Spectral component separation. Pass energy: $23.5 \mathrm{eV}$. The contributions from oxygen bound to carbon and $\mathrm{Zn}(\mathrm{OH}) 2$ are not separated, therefore, the decomposition of the carbon $\mathrm{C} 1 \mathrm{~s}$ peak is shown below in Table 5 . An error of $5 \%$ was assumed. Due to a strong overlap of the Si2p peak with a shake-up from $\mathrm{Zn}$, the $\mathrm{Si}$ content could not be determined as reliably as the other values. For the determination of the given value, a maximum interval of binding energies was chosen to avoid underestimation.

\begin{tabular}{lcccccccc}
\hline $\begin{array}{l}\text { Peak } \\
\text { Compound }\end{array}$ & $\mathrm{Zn}$ & $\begin{array}{c}\mathrm{Zn2p}_{\mathbf{3} / 2} \\
\mathrm{ZnO}\end{array}$ & $\mathrm{Zn}(\mathrm{OH})_{2}$ & $\mathrm{SiO}_{2}$ & $\begin{array}{c}\text { O1s } \\
\mathrm{Zn}(\mathrm{OH})_{2} \\
\mathrm{C} \text { comp. }\end{array}$ & $\mathrm{ZnO}$ & C1s & Si2p \\
\hline $\begin{array}{l}\text { Peak pos. } \\
\text { [eV] }\end{array}$ & 1021.59 & 1022.19 & 532.70 & 531.87 & 530.59 & & 103.29 \\
$\begin{array}{l}\text { At. perc. } \\
\text { [\%] }\end{array}$ & 0 & 21.5 & 5.9 & 9.9 & 15.8 & 22.0 & 19.3 & $(5.6)$ \\
\hline
\end{tabular}


Table 5: Pristine surface: spectral component separation of the carbon C1s peak. Pass energy: $23.5 \mathrm{eV}$. An error of $5 \%$ was assumed.

\begin{tabular}{lccccc}
\hline $\begin{array}{l}\text { Peak no. } \\
\text { Compound }\end{array}$ & $\begin{array}{c}\text { A } \\
\text { C-C, C-H }\end{array}$ & $\begin{array}{c}\text { B } \\
\text { C-OH, C-O-C }\end{array}$ & $\begin{array}{c}\text { C }=\mathrm{O} \\
\text { C }\end{array}$ & $\begin{array}{c}\text { D } \\
\text { O-C=O }\end{array}$ & $\begin{array}{c}\text { E } \\
-\mathrm{CO}_{3}\end{array}$ \\
\hline $\begin{array}{l}\text { Peak pos. } \\
{[\mathrm{eV}]}\end{array}$ & 284.88 & 286.39 & 288.13 & 289.11 & 290.11 \\
At. perc. $[\%]$ & 14.0 & 1.3 & 0.8 & 2.2 & 1.0 \\
\hline
\end{tabular}

Table 6: Etched surface (16 min): spectral component separation. Pass energy: $23.5 \mathrm{eV}$. An error of $5 \%$ was assumed.

\begin{tabular}{lcccccccc}
\hline $\begin{array}{l}\text { Peak } \\
\text { Compound }\end{array}$ & $\mathrm{Zn}$ & $\begin{array}{c}\mathrm{Zn2p}_{3 / 2} \\
\mathrm{ZnO}\end{array}$ & $\mathrm{Zn}(\mathrm{OH})_{2}$ & $\mathrm{SiO}_{2}$ & $\begin{array}{c}\mathbf{\mathrm { O }} \mathrm{\textrm {Zn }}(\mathrm{OH})_{2} \\
\mathrm{C} \text { comp. }\end{array}$ & $\mathrm{ZnO}$ & C1s & Si2p \\
\hline $\begin{array}{l}\text { Peak pos. } \\
\text { [eV] }\end{array}$ & 1020.56 & 1021.13 & & 532.60 & & 529.91 & & 103.31 \\
$\begin{array}{l}\text { At. perc. } \\
\text { [\%] }\end{array}$ & 6.1 & 4.6 & 0 & 55.9 & 0 & 4.6 & 1.1 & 27.8 \\
\hline
\end{tabular}

It turns out that the peak positions of the $\mathrm{O} 1 \mathrm{~s}$ peak (metallic bound) and the $\mathrm{Zn} 2 \mathrm{p}_{3 / 2}$ peak (oxygen bound) before and after etching do not entirely coincide (cf. Table 4 and Table 6). This is assumed to be due to lattice relaxations in $\mathrm{ZnO}$ following the argon bombardment, leading to a conductivity [46] which yields slightly different binding energies. The resulting peak positions are in good agreement with the reference spectra (cf. Table 3). Since the reference spectra were shifted by means of the LMM positions of the etched coating, the corresponding O1s (metallic bound) and $\mathrm{Zn} 2 \mathrm{p}_{3 / 2}$ (elemental $\mathrm{Zn}$ as well as $\mathrm{ZnO}$ contribution) peak positions in the reference spectra concur well with the positions for the etched coating. For the pristine sample, however, these positions are shifted towards higher binding energies. $\mathrm{SiO}_{2}$ is less influenced by this effect [36]; consistently, the position of the O1s peak resulting from the $\mathrm{Si}-\mathrm{O}$ bond in $\mathrm{SiO}_{2}$ does not shift.

In summary, XPS analysis confirmed the existence of a thin, almost closed layer of nano-sized zinc oxide particles located between larger agglomerates which are composed of a mixed system consisting of $\mathrm{Zn}$ and $\mathrm{ZnO}$. The plasma deposition process in general induced oxidation of the original zinc powder, hence, the presented method can be used to create $\mathrm{ZnO}$ coatings on thermosensitive materials at atmospheric pressure.

\section{Conclusions}

Our analysis gives an overview on structural properties of coatings based on zinc powder deposited by the specifically used cold plasma-spray setup. As ascertained by LSM and AFM measurements, the coating partially consists of a number of several large particles/agglomerates with a diameter of several microns. Even though the diameter of the raw material, i. e. the Zn powder, was $\mathrm{d}_{50}=13 \mu \mathrm{m}$, an almost closed basic layer of very small, nanometre-sized particles in between such larger particles/agglomerates was detected. The existence of particles in the nanoscale could be due to melting and vaporisation of initial particles within the hot plasma zone and a selective deposition of an almost closed layer of small particles and some agglomerated big clusters. Given this behaviour relatively large powder particles could be applied as raw material for realising nanoparticle coatings.

It can further be assumed that the plasma deposition process gives rise to an increased oxidation of zinc particles since the degree of oxidation is much higher than expected in the case of regular oxidation at ambient air. An increased oxidation can be explained by the excitation and/or dissociation of oxygen molecules from the used process gas and was confirmed by XPS depth profiles of the elemental composition. Here, a predominantly $\mathrm{Zn}$-based pristine surface and a quick decrease in zinc with increasing sputter time, which results in a constant portion of $\mathrm{Zn}$, was observed. The existence of $\mathrm{Zn}(\mathrm{OH})_{2}$ at the pristine surface was also measured, whereas this bond was not found on the etched surface. The surface etched for 16 minutes consists of $\mathrm{ZnO}$ and elemental $\mathrm{Zn}$ in approximately equal shares. To our best knowledge, this is the first work presenting quantitative analysis of a mixed system 
consisting of $\mathrm{Zn}$ and $\mathrm{ZnO}$ where the main challenge originates from the overlap of the $\mathrm{Zn} 2 \mathrm{p}_{3 / 2}$ peak which applies for both compounds.

The presence of $\mathrm{ZnO}$ within the layer is also indicated by transmittance spectra where a band gap energy of $(3.20 \pm 0.02) \mathrm{eV}$ was determined. This value is in good agreement with literature. Furthermore, the strong UV absorbance of very thin coatings was shown. Based on these results and the simple and easy-to-use working principle of the presented cold plasma-spray process a wide range of possible applications can be suggested.

The deposited coatings could be used as UV protection layer on wood surfaces or other appropriate substrate materials. Since the substrate heating is relatively low, thermosensitive materials can be coated when using the presented method. Another potential application of this approach is the realisation of optical coatings, e. g. UV blocking filters for protection windows as used in laser safety or welding practice. Furthermore, zinc oxide has a certain biocidal function and (amongst other effects) inhibits the uptake of manganese which is essential for a number of bacteria or fungi. Thus, the presented method could be an approach for the realisation of anti-bacterial layers.

The results suggest that atmospheric pressure plasma deposition of zinc particle coatings is a promising technique with a number of potential fields of applications. However, the description of the dependency of the large number of possible, partially inter-acting plasma parameters and the resulting coating properties still requires extensive studies. For instance, systematic investigation of the impact of process parameters on the band gap energy, the structural coating properties and the chemical composition should be performed. Relating to the above-mentioned applications, the evaluation of long-term stability and functionality of deposited coatings is further of essential interest. These open points will be addressed in ongoing and future work.

\section{Acknowledgements}

This work was carried out within the framework of the research project "PlaNaWood" (funding reference no. 03X5519B) with financial support from the German Federal Ministry of Education and Research. The authors further thank ECKART GmbH for providing the used powder material and Roger Skarsten for proofreading the manuscript.

\section{References}

[1] H. Marchebois, S. Joiret, C. Savall et al., "Characterization of zinc-rich powder coatings by EIS and Raman spectroscopy," Surface and Coatings Technology, no. 157, pp. 151-161, 2002.

[2] J. Wang, Z. Wang, B. Huang et al., "Oxygen vacancy induced band-gap narrowing and enhanced visible light photocatalytic activity of ZnO," ACS Applied Materials \& Interfaces, vol. 4, no. 8, pp. 4024-4030, 2012.

[3] E. A. Dalchiele, P. Giorgi, R. E. Marotti et al., "Electrodeposition of $\mathrm{ZnO}$ thin films on n-Si (100)," Solar Energy Materials and Solar Cells, vol. 70, no. 3, pp. 245-254, 2001.

[4] P. L. Washington, H. C. Ong, J. Y. Dai et al., "Determination of the optical constants of zinc oxide thin films by spectroscopic ellipsometry," Applied Physics Letters, vol. 72, no. 25, pp. 3261-3263, 1998.

[5] D. Gal, G. Hodes, D. Lincot et al., "Electrochemical deposition of zinc oxide films from nonaqueous solution: a new buffer/window process for thin film solar cells," Thin Solid Films, 361362, pp. 79-83, 2000.

[6] C. A. Clausen, F. Green, and S. Nami Kartal, "Weatherability and leach resistance of wood impregnated with nano-zinc oxide," Nanoscale Research Letters, vol. 5, no. 9, pp. 1464-1467, 2010.

[7] Y. Yu, Z. Jiang, G. Wang et al., "Growth of $\mathrm{ZnO}$ nanofilms on wood with improved photostability," Holzforschung, vol. 64, no. 3, pp. 385-390, 2010.

[8] C. A. McDevitt, A. D. Ogunniyi, E. Valkov et al., "A molecular mechanism for bacterial susceptibility to zinc," PLoS Pathogens, vol. 7, no. 11, e1002357, 2011.

[9] S. B. Pointing, V. Bucher, and L. Vrijmoed, "Dye decolorization by sub-tropical basidiomycetous fungi and the effect of metals on decolorizing ability," World Journal of Microbiology and Biotechnology, vol. 16, no. 2, pp. 199-205, 2000. 
[10] H. Yasuda, "Glow discharge polymerization," Journal of Polymer Science: Macromolecular Reviews, vol. 16, no. 1, pp. 199-293, 1981.

[11] L. Podgorski, C. Bousta, F. Schambourg et al., "Surface modification of wood by plasma polymerisation," Pigment \& Resin Technology, vol. 31, no. 1, pp. 33-40, 2002.

[12] M. Bente, G. Avramidis, S. Förster et al., "Wood surface modification in dielectric barrier discharges at atmospheric pressure for creating water repellent characteristics," Holz als Rohund Werkstoff, vol. 62, no. 3, pp. 157-163, 2004.

[13] G. Toriz, M. G. Gutiérrez, V. González-Alvarez et al., "Highly hydrophobic wood surfaces prepared by treatment with atmospheric pressure dielectric barrier discharges," Journal of Adhesion Science and Technology, vol. 22, no. 16, pp. 2059-2078, 2008.

[14] S. Zanini, C. Riccardi, M. Orlandi et al., "Wood coated with plasma-polymer for water repellence," Wood Science and Technology, vol. 42, no. 2, pp. 149-160, 2008.

[15] G. Avramidis, E. Hauswald, A. Lyapin et al., "Plasma treatment of wood and wood-based materials to generate hydrophilic or hydrophobic surface characteristics," Wood Material Science \& Engineering, vol. 4, 1-2, pp. 52-60, 2009.

[16] J. Profili, O. Levasseur, A. Koronai et al., "Deposition of nanocomposite coatings on wood using cold discharges at atmospheric pressure," Surface and Coatings Technology, vol. 309, pp. 729737, 2017.

[17] P. Gascón-Garrido, N. Mainusch, H. Militz et al., "Copper and aluminium deposition by coldplasma spray on wood surfaces: effects on natural weathering behaviour," European Journal of Wood and Wood Products, vol. 75, no. 3, pp. 315-324, 2016.

[18] P. Gascón-Garrido, N. Mainusch, H. Militz et al., "Effects of copper-plasma deposition on weathering properties of wood surfaces," Applied Surface Science, vol. 366, pp. 112-119, 2016.

[19] L. M. Wallenhorst, S. Dahle, M. Vovk et al., "Characterisation of PMMA/ATH layers realised by means of atmospheric pressure plasma powder deposition," Advances in Condensed Matter Physics, vol. 2015, no. 3, pp. 1-12, 2015.

[20] "Kunststoffe - Dynamische Differenz-Thermoanalyse (DSC) - Teil 2: Bestimmung der Glasübergangstemperatur und der Glasübergangsstufenhöhe," 2014, DIN EN ISO 11357-2.

[21] ZEN black, Carl Zeiss Microscopy GmbH, Jena, Germany.

[22] D. Nečas and P. Klapetek, "Gwyddion: An open-source software for SPM data analysis," Open Physics, vol. 10, no. 1, 2012.

[23] "Solar energy; Reference solar spectral irradiance at the ground at different receiving conditions; Part 1: Direct normal and hemispherical solar irradiance for air mass 1,5," 1992, ISO 9845$1: 1992$.

[24] MultiPak, Physical Electronics Inc., Chanhassen, USA.

[25] F. Wooten, Optical Properties of Solids, Academic Press, New York, 1972.

[26] S. Maensiri, P. Laokul, and V. Promarak, "Synthesis and optical properties of nanocrystalline ZnO powders by a simple method using zinc acetate dihydrate and poly(vinyl pyrrolidone)," Journal of Crystal Growth, vol. 289, no. 1, pp. 102-106, 2006.

[27] V. Srikant and D. R. Clarke, "Optical absorption edge of $\mathrm{ZnO}$ thin films: The effect of substrate," Journal of Applied Physics, vol. 81, no. 9, pp. 6357-6364, 1997.

[28] J. F. Moulder, W. F. Stickle, P. E. Sobol et al., Handbook of X-Ray Photoelectron Spectroscopy: A Reference Book of Standard Spectra for Identification and Interpretation of XPS Data, Physical Electronics Division, Japan, 1992.

[29] M. C. Biesinger, L. W. M. Lau, A. R. Gerson et al., "Resolving surface chemical states in XPS analysis of first row transition metals, oxides and hydroxides: Sc, Ti, V, Cu and Zn," Applied Surface Science, vol. 257, no. 3, pp. 887-898, 2010.

[30] G. Schön, "Auger and direct electron spectra in X-ray photoelectron studies of zinc, zinc oxide, gallium and gallium oxide," Journal of Electron Spectroscopy and Related Phenomena, no. 2, pp. 75-86, 1973.

[31] K.-J. Qian, S. Chen, B. Zhu et al., "Atomic layer deposition of $\mathrm{ZnO}$ on thermal $\mathrm{SiO}_{2}$ and $\mathrm{Si}$ surfaces using $\mathrm{N}_{2}$-diluted diethylzinc and $\mathrm{H}_{2} \mathrm{O}_{2}$ precursors," Applied Surface Science, vol. 258, no. 10, pp. 4657-4666, 2012.

[32] Y. Chen, P. Schneider, and A. Erbe, "Investigation of native oxide growth on zinc in different atmospheres by spectroscopic ellipsometry," Physica Status Solidi A, vol. 209, no. 5, pp. 846$853,2012$. 
[33] C. Wöll, "The chemistry and physics of zinc oxide surfaces," Progress in Surface Science, vol. 82, 2-3, pp. 55-120, 2007.

[34] S. Hofmann, "Quantitative depth profiling in surface analysis: A review," Surface and Interface Analysis, vol. 2, no. 4, pp. 148-160, 1980.

[35] S. Hofmann and J. M. Sanz, "Quantitative Erfassung des Ionenstrahleinflusses beim Sputtering von Oxidschichten mit AES und XPS," Fresenius Zeitschrift für Analytische Chemie, vol. 314, no. 3, pp. 215-219, 1983.

[36] M. P. Seah and T. S. Nunney, "Sputtering yields of compounds using argon ions," Journal of Physics D: Applied Physics, vol. 43, no. 25, p. 253001, 2010.

[37] D. F. Mitchell, G. I. Sproule, and M. J. Graham, "Sputter reduction of oxides by ion bombardment during Auger depth profile analysis," Surface and Interface Analysis, vol. 15, no. 8, pp. 487-497, 1990.

[38] R. Kelly, "On the problem of whether mass or chemical bonding is more important to bombardment-induced compositional changes in alloys and oxides," Surface Science, vol. 100, no. 1 , pp. 85-107, 1980.

[39] C. A. Gouvêa, F. Wypych, S. G. Moraes et al., "Semiconductor-assisted photocatalytic degradation of reactive dyes in aqueous solution," Chemosphere, vol. 40, no. 4, pp. 433-440, 2000.

[40] A. McLaren, T. Valdes-Solis, G. Li et al., "Shape and size effects of $\mathrm{ZnO}$ nanocrystals on photocatalytic activity," Journal of the American Chemical Society, vol. 131, no. 35, pp. 1254012541, 2009.

[41] A. Rezaee, H. Rangkooy, A. Khavanin et al., "High photocatalytic decomposition of the air pollutant formaldehyde using nano-ZnO on bone char," Environmental Chemistry Letters, vol. 12, no. 2, pp. 353-357, 2014.

[42] H. F. Moafi, A. F. Shojaie, and M. A. Zanjanchi, "Photocatalytic self-cleaning properties of cellulosic fibers modified by nano-sized zinc oxide," Thin Solid Films, vol. 519, no. 11, pp. 3641-3646, 2011.

[43] J. Hirschberg, C. Gerhard, A. Braun et al., "Validation of the suitability of stripped lipid as a skin model in plasma medical investigations," Open Journal of Applied Sciences, vol. 05, no. 02, pp. 40-49, 2015.

[44] K.-D. Weltmann, E. Kindel, R. Brandenburg et al., "Atmospheric pressure plasma jet for medical therapy: Plasma Parameters and Risk Estimation," Contributions to Plasma Physics, vol. 49, no. 9, pp. 631-640, 2009.

[45] B. P. Payne, M. C. Biesinger, and N. S. McIntyre, "X-ray photoelectron spectroscopy studies of reactions on chromium metal and chromium oxide surfaces," Journal of Electron Spectroscopy and Related Phenomena, vol. 184, 1-2, pp. 29-37, 2011.

[46] A. Janotti and van de Walle, C. G., "Oxygen vacancies in ZnO," Applied Physics Letters, vol. 87, no. 12 , p. 122102, 2005. 


\title{
Paper II
}

\section{UV-Blocking Properties of Zn/ZnO Coatings on Wood deposited by Cold Plasma Spraying at Atmospheric Pressure}

\author{
L. Wallenhorst ${ }^{1}$, L. Gurău², A. Gellerich ${ }^{3}$, H. Militz ${ }^{3}$, G. Ohms ${ }^{1}$, W. Viöl ${ }^{1,4}$ \\ ${ }^{1}$ University of Applied Sciences and Arts, Laboratory of Laser and Plasma Technologies, \\ Von-Ossietzky-Straße 99, 37085 Göttingen, Germany \\ ${ }^{2}$ Transilvania University of Braşov, Faculty of Wood Engineering, Str. Universităţii nr. 1, corp L, \\ Braşov, Romania
}

${ }^{3}$ University of Göttingen, Wood Biology and Wood Products, Faculty of Forest Sciences, Büsgenweg 4, 37075 Göttingen, Germany

${ }^{4}$ Fraunhofer Institute for Surface Engineering and Thin Films, Application Center for Plasma and Photonics, Von-Ossietzky-Str. 100, 37085 Göttingen, Germany

\begin{abstract}
In this study, artificial ageing of beech wood coated with $\mathrm{Zn} / \mathrm{ZnO}$ particles by means of a cold plasma spraying process as well as coating systems including a $\mathrm{Zn} / \mathrm{ZnO}$ layer and additional conventional sealings were examined. As ascertained by colour measurements, the particle coatings significantly decreased UV light-induced discolouration. Even though no significant colour changes were observed for particle-coated and alkyd-sealed samples, ATR-FTIR measurements revealed photocatalytic degradation of the alkyd matrix. In contrast, the polyurethane sealing appeared to be stabilised by the $\mathrm{Zn} / \mathrm{ZnO}$ coating. Furthermore, morphologic properties of the pure particle coatings were studied by SEM and roughness measurements. SEM measurements confirmed a melting and solidifying process during deposition.
\end{abstract}

Keywords: Photostabilisation, Wood, Plasma Coatings, Zinc Oxide, ATR-FTIR, Surface Roughness

\section{Introduction}

As one of the oldest construction materials, wood is gaining even more importance with respect to contemporary demands for sustainability. Hence, the further development of wood products plays a major role in material science. In outdoor use however, wood and wood products are subjected to biotic and abiotic damage such as degradation by UV light. Many tropical wood exhibits a good resistance to biotic degradation mechanisms, yet its limited availability and the negative connotation regarding the destruction of tropical forests make its use undesirable. In contrast, most of the temperate wood species show a poor dimensional stability when faced with changes in temperature and humidity, a limited resistance to fungi and bacteria [1,2], and a particularly high sensitivity to UV light $[1,3-5]$.

Consequently, the development of highly efficient protective wood coatings is the subject of intense current research. Particularly to protect wood from photodegradation, inorganic UV absorbers such as $\mathrm{ZnO}$ and $\mathrm{TiO}_{2}$ have been widely studied and have proven to successfully protect the surface [6-9]. The use of (nano-) particles may, however, lead to an unintentional release of nanoparticles caused e. g. by abrasion due to airborne sand particles. These nanoparticles may be harmful for both human health and the (aquatic) environment $[10,11]$. Another challenge is represented by the photocatalytic activity of many UV absorbers that can lead to the degradation of organic matrix materials [12-16].

Among the emerging wood coating techniques are plasma-assisted processes. Various studies have addressed the generation of hydrophobic plasma polymers [17-20]. Furthermore, a plasma can be used 
to directly deposit solid powder material even on thermosensitive materials like wood [21-24]. In a previous study [24], thin particle coatings based on elemental Zn powder were deposited on glass substrates. Their characterisation revealed a mixed system of $\mathrm{Zn}$ and $\mathrm{ZnO}$ which led to a significantly reduced transmittance of light in the UV range. Based on these results, the present study investigates UV-blocking properties of similar coatings on beech wood (here referred to as "particle coating"). Additionally, coating systems composed of a particle coating and a conventional sealing (alkyd/polyurethane) were studied. The sequential application of UV absorbers and a sealing permits a precise dosage of UV absorbers and should at the same time inhibit nanoparticle release due, for example, to abrasion.

In this study, the UV-protective properties of $\mathrm{Zn} / \mathrm{ZnO}$ coatings deposited in a cold plasma spraying process on beech wood were examined. In addition to the pure particle coatings, two coating systems involving a particle coating and a conventional top sealing (alkyd or polyurethane) were tested to eventually develop a coating system that permits a precise dosage of these UV absorbers and inhibits nanoparticle release from both leaching and abrasion. The effect of UV irradiation on the surfaces was assessed by colour and ATR-FTIR measurements.

Moreover, the morphology of such coatings was studied by means of SEM and surface roughness measurements.

\section{Materials and Methods}

\section{Materials}

To carry out the UV tests, different coating systems were applied to beech wood samples (Fagus sylvatica L.) with dimensions $15 \times 7 \times 5 \mathrm{~cm}^{3}$. In addition to the reference samples, $\mathrm{Zn} / \mathrm{ZnO}$ coatings were deposited by cold plasma spraying as described below. Since the pure $\mathrm{Zn} / \mathrm{ZnO}$ coatings do not exhibit a sufficient leach resistance, two commercially available sealing systems were tested along with unsealed samples. The used alkyd paint (ProfiDur Klarlack, Schöner Wohnen Farbe) represents a standard outdoor paint for wooden surfaces. Furthermore, the polyurethane (PUR) SKresin P34 NV from S u. K Hock GmbH was applied to investigate a second type of coating system. Three replicates were prepared for each coating system.

To study the roughness of particle-coated samples, one concentration (coating B, see below) of particles was also applied to beech wood samples of the size $6 \times 10 \times 4 \mathrm{~cm}^{3}$ as well as to polypropylene samples $\left(6 \times 10 \times 3 \mathrm{~cm}^{3}\right)$.

Zinc particles with a diameter $\mathrm{d}_{50}=16 \mu \mathrm{m}$ were provided by ECKART GmbH. These particles feature an irregular shape and were found to improve the coating's homogeneity compared to the flake-like particles that were used previously [24].

\section{Deposition process}

The particle coatings were applied by means of a setup as described earlier [21-24]. In short, dry Zn particles were added to the afterglow of a jet discharge operated at atmospheric pressure while the sample was moved below the spraying nozzle by a xy linear stage to ensure an overall coating. The plasma source had an ignition voltage of approx. $15 \mathrm{kV}$ and an effective voltage of $2-3 \mathrm{kV}$. Its maximum input power amounted to $2 \mathrm{~kW}$ and pulses were generated at a frequency of $50 \mathrm{kHz}$ with a duration of 5-10 $\mu \mathrm{s}$. Compressed air was used as the process gas. To generate a particle aerosol, the uniformly compressed powder was moved towards a rotating brush at a constant velocity which defined the feed rate. Finally, an air stream took up the powder above the brush to carry the particle aerosol towards the discharge. The carrier gas pressure was set to $0.16 \mathrm{MPa}$. During the deposition, the process gas flow was kept constant at $30 \mathrm{~L} / \mathrm{min}$. The distance between the spraying nozzle and the sample amounted to $16 \mathrm{~mm}$.

Table 1 lists parameters for the two coatings that were varied to obtain two different concentrations. This specific plasma source significantly limits substrate heating compared to conventional plasma spraying and therefore permits coating of thermosensitive materials. On the other hand, it restricts applicable powders to those featuring a melting temperature of less than approx. $1500^{\circ} \mathrm{C}$. Therefore, zinc particles were used as the base material instead of directly depositing $\mathrm{ZnO}$ powder. 
Table 1: Summary of varied coating parameters to generate a thin coating (A) and a thick coating (B).

\begin{tabular}{lcc}
\hline Coating No. & $\begin{array}{c}\text { Powder feed rate } \\
\text { in } \mathbf{~ c m}^{3} \mathbf{h}\end{array}$ & $\begin{array}{c}\text { Substrate displacement } \\
\text { in } \mathbf{~ m m} / \mathbf{s}\end{array}$ \\
\hline $\mathrm{A}$ & 16.1 & 80 \\
$\mathrm{~B}$ & 24.1 & 50 \\
\hline
\end{tabular}

\section{Morphologic characterisation}

Roughness measurements were performed for uncoated (kept as control) as well as particle-coated surfaces (coating B) of beech specimens $\left(6 \times 10 \times 4 \mathrm{~cm}^{3}\right.$, previously processed by planning), as well as for polypropylene samples $\left(6 \times 10 \times 3 \mathrm{~cm}^{3}\right)$. Wood is an example of a surface with inherent anatomical irregularities (for example wood pores), while polypropylene is a homogeneous material chosen for comparisons of the effect of the particle coating on surface morphology. For the roughness measurements, a MarSurf XT20 measuring system with XT 20 Topography manufactured by MAHR Göttingen GmbH was used. The instrument was endowed with a scanning head MFW 250 with tracing arm in the range of $\pm 500 \mu \mathrm{m}$ and a stylus with $2 \mu \mathrm{m}$ tip radius and $90^{\circ}$ tip angle. The specimens were measured at a speed of $0.5 \mathrm{~mm} / \mathrm{s}$, at a vertical resolution of $7 \mathrm{~nm}$, a lateral resolution of $1 \mu \mathrm{m}$, and with a low scanning force of $0.7 \mathrm{mN}$. Both for coated and uncoated materials, five replicates were measured by randomly tracing three profiles per sample with a length of $70 \mathrm{~mm}$, so that 15 profiles were available for evaluation of each combination of treatment and material. In the case of beech specimens, the measurements were carried out perpendicular to the grain direction, which corresponds to the direction of the highest roughness [25].

Roughness represents the finer irregularities of the surface texture that are usually inherent in a machining process and represented the target of this study in order to see by comparison with reference samples if any modification in surface morphology was caused by treating the surface with zinc particles. In order to obtain the surface roughness, the measured profiles must be filtered from other types of irregularities. In this sense, a Robust Gaussian Regression Filter contained in ISO/TS 16610-31:2010 [26] was used. This filter was tested and found suitable for wood surfaces [27,28].

After filtering, a range of roughness parameters were calculated for the profiles, such as: $R a, R q, R t$, from ISO 4287 [29] and $R k, R p k, R v k$ from ISO 13565-2 [30]. $R a$ and $R q$ are mean parameters (the arithmetic mean of the absolute ordinate values, $R a$, and the root mean square value of the ordinate values, $R q$ ) most commonly used. $R t$ is the total height of the profile calculated as the sum of the maximum profile peak height and the largest absolute value profile valley depth and is expected to be sensitive to variations in local wood anatomy. $R k$ measures the core roughness of a profile, is the parameter least biased by inherent variation in wood anatomy and should best indicate the modifications in roughness caused by coating the surface. Rpk is the reduced peak height and represents the average height of the protruding peaks above the core roughness profile. $R v k$ is the reduced valley depth and represents the average depth of the profile valleys projecting through the core roughness profile. Both parameters are sensitive to outlying irregularities, as fuzziness in the case of Rpk and outlying deep anatomical valleys in the case of Rvk [31].

For treated and untreated surfaces and each roughness parameter, a mean value and the standard deviation were calculated. ANOVA with single factor was performed to test significant differences between reference samples and those treated with zinc particles.

Moreover, the particle coatings' morphology was examined by Scanning Electron Microscopy (SEM, JSM-5600LV from Jeol USA, Inc). All samples were sputter coated with a thin (approx. $32 \mathrm{~nm}$ ) gold layer and measured at an accelerating voltage of 12 or $15 \mathrm{kV}$.

\section{Artificial ageing}

Artificial ageing was simulated by a chamber for artificial weathering (QUV/Spray from Q-Lab Corporation) without using the spraying system and at a constant temperature of $50^{\circ} \mathrm{C}$. The solar irradiation intensity was set to $0.89 \mathrm{~W} /\left(\mathrm{m}^{2} \mathrm{~nm}\right)$ at the lamp's emission peak around $340 \mathrm{~nm}$ $(290 \mathrm{~nm} \leq \lambda \leq 400 \mathrm{~nm})[32]$. Three replicates were prepared for each coating system.

Colour measurements were performed by taking pictures under equal conditions and extracting colour parameters with the software Photoshop CS6. Constancy of illumination was confirmed by 
measurements with the spectroradiometer CS-200A (Konica Minolta). CIE Lab parameters were calculated on all three samples. In this colour system, $L$ quantifies the lightness in grey-scale values from 0 to 100, whereas $a$ and $b$ represent the two colour axes. $\Delta \mathrm{a}=\mathrm{a}_{0}-\mathrm{a}_{\mathrm{i}}>0$ represents a green shift for the $i$-th measurement, $\Delta \mathrm{a}<0$ a red shift, $\Delta \mathrm{b}=\mathrm{b}_{0}-\mathrm{b}_{\mathrm{i}}>0$ a blue shift and $\Delta \mathrm{b}<0$ a yellow shift. The overall colour changes can be quantified by:

$$
\Delta E=\sqrt{\left(L_{0}-L_{i}\right)^{2}+\left(a_{0}-a_{i}\right)^{2}+\left(b_{0}-b_{i}\right)^{2}} .
$$

Chemical changes caused by UV irradiation were examined by Attenuated Total Reflectance Fourier Transform Infrared Spectroscopy (ATR-FTIR, Perkin Elmer Frontier). Spectra were acquired for wavelengths ranging from 4000 to $400 \mathrm{~cm}^{-1}$ by performing 32 scans at a resolution of $4 \mathrm{~cm}^{-1}$. All spectra were ATR and baseline corrected as well as normalised by use of the software Spectrum from Perkin Elmer. Moreover, slight smoothing was applied using a Savitzky-Golay filter $\left(2^{\text {nd }}\right.$ degree polynomial). Measurements were carried out at identical spots before and after irradiation. For every coating system, a minimum of three measurements was obtained.

\section{Results and Discussion}

Morphology of unsealed coatings

The surface roughness results are summarised in Table 2 and Table 3.

Table 2: Roughness parameters (mean values) and standard deviations for untreated and particle-coated beech surfaces (values in microns).

\begin{tabular}{lcccccc}
\hline Sample & $\boldsymbol{R a}$ & $\boldsymbol{R q}$ & $\boldsymbol{R t}$ & $\boldsymbol{R k}$ & $\boldsymbol{R p k}$ & $\boldsymbol{R} \boldsymbol{k}$ \\
\hline Reference beech & 5.7 & 8.6 & 72.3 & 12.0 & 6.5 & 18.4 \\
stdev & 0.30 & 0.65 & 8.11 & 0.92 & 0.90 & 1.56 \\
& & & & & & \\
Particle-coated beech & 6.2 & 9.1 & 80.4 & 13.67 & 9.12 & 17.89 \\
stdev & 0.24 & 0.48 & 7.50 & 1.66 & 1.63 & 2.22 \\
\hline
\end{tabular}

Table 3 Roughness parameters (mean values) and standard deviation for untreated and particle-coated polypropylene surfaces (values in microns).

\begin{tabular}{lcccccc}
\hline Sample & $\boldsymbol{R} \boldsymbol{a}$ & $\boldsymbol{R q}$ & $\boldsymbol{R} \boldsymbol{t}$ & $\boldsymbol{R} \boldsymbol{k}$ & $\boldsymbol{R p k}$ & $\boldsymbol{R} \boldsymbol{k}$ \\
\hline Reference polypropylene & 0.019 & 0.028 & 1.101 & 0.056 & 0.027 & 0.038 \\
stdev & 0.001 & 0.009 & 0.837 & 0.003 & 0.005 & 0.015 \\
& & & & & & \\
Particle-coated polypropylene & 5.325 & 7.370 & 61.622 & 14.068 & 12.405 & 2.098 \\
stdev & 0.287 & 0.343 & 9.417 & 1.385 & 0.703 & 1.115 \\
\hline
\end{tabular}

From Table 2, it can be seen that the particle coating increased the surface roughness of beech. This increase is more reliably evaluated by the $R k$ parameter, because it is the parameter least biased by beech wood anatomy [33]. $R k$ increased with $13.6 \%$ in the case of particle-coated beech and this difference was significant for a $95 \%$ confidence interval $(\mathrm{p}<0.05)$ according to the ANOVA test. In absolute values, this means a surface $1.64 \mu \mathrm{m}$ rougher if coated. The fact that the surface was coated has generated, as expected, an increase in surface peaks as measured by the parameter Rpk, which exhibited the highest increase (41\%) for coated beech compared to untreated surfaces. $R v k$, which may be a measure of wood anatomical irregularities (mostly wood pores) for uncovered wood, decreased by $2.6 \%$ as compared to untreated beech, which is an indication that the particles may have penetrated into the wood anatomical cavities and obscuring them to a certain extent.

From Table 3, it can be observed that the particle coating caused a large increase in surface roughness of plastic surfaces for all measured parameters. For example, the $R k$ parameter increased 249 times, but the highest increase was recorded again for $R p k$ (461 times), while $R v k$ exhibited the minimum increase (55 times). This indicates that the particle coating increased the occurrence of isolated peaks on the surface for both polypropylene and beech surfaces. In comparison with polypropylene, the particles penetrate into the wood anatomical irregularities (gaps) and its overall effect on surface roughness is small. 
To further study the coatings' morphology, SEM measurements were acquired. Figure 1 shows representative images of the unmodified powder as well as the surfaces of both coating A and B (frame size: $48 \times 64 \mu \mathrm{m}^{2}$ ).

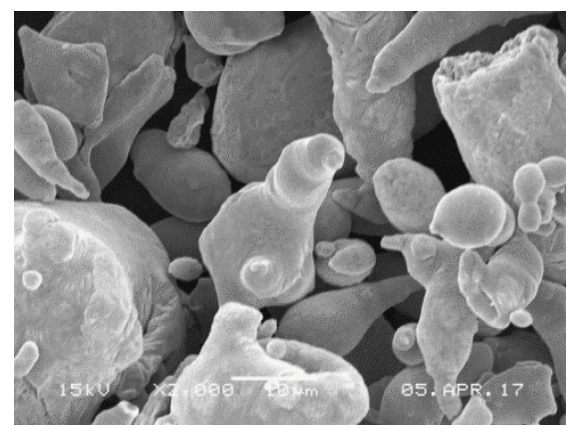

(a)

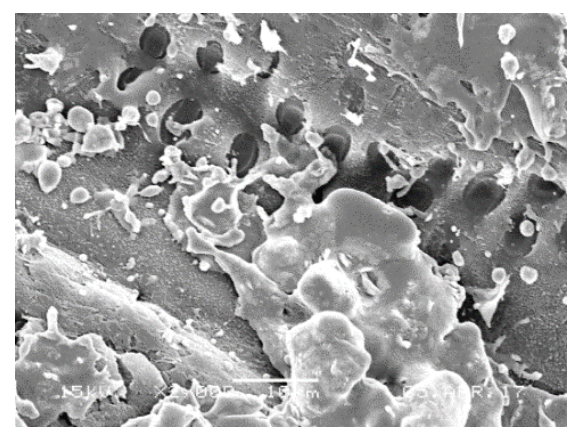

(b)

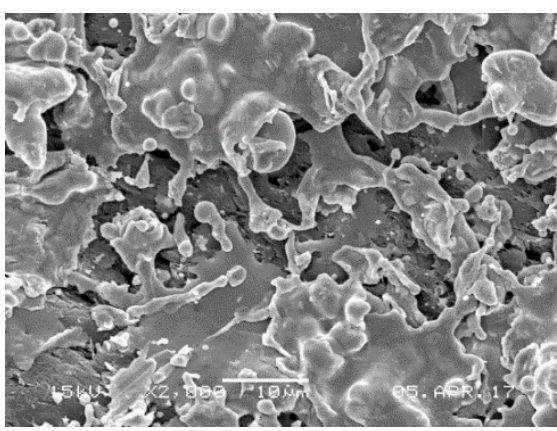

(c)

Figure 1: SEM measurements on (a) untreated powder, (b) thin coated (A) and (c) thick coated (B) sample.

For both coatings, the particles' shape differs significantly from the shape of the unmodified particles, confirming a melting and solidifying process as generally expected for plasma spraying techniques. In particular for thin coated samples (Figure 1(b), the wood structure was still clearly visible but even the thick coating did not completely obscure the original surface. Figure 2 shows representative images of cross sections of the coatings confirming the formation of very flat particle shapes with thicknesses around or below $1 \mu \mathrm{m}$. The coating, being composed of several such particles with different thicknesses, appears to feature thicknesses around a few $\mu \mathrm{m}$ (approximately up to $5 \mu \mathrm{m}$ ).

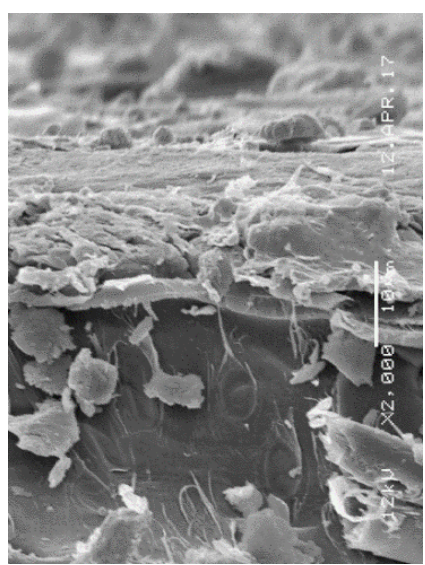

(a)

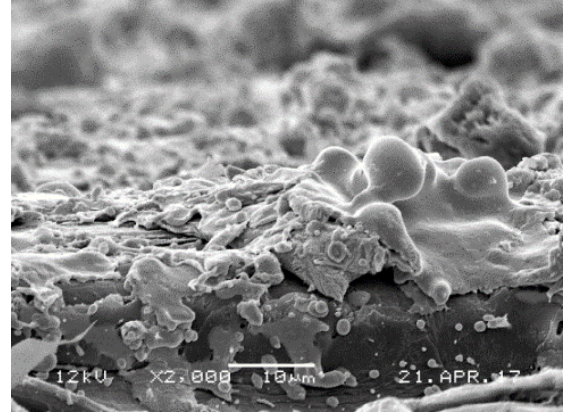

(b)

Figure 2: SEM measurements of cross sections. (a): Thin coating A, (b): thick coating B. 
Colour changes induced by UV irradiation

All samples were exposed to UV radiation for a total of $50 \mathrm{~h}$ with irradiation intervals ranging from 2 to 10 hours. After each interval, pictures were taken to analyse colour changes.

The evolution of the total colour change $\Delta E$ of the different coating systems with increasing UV irradiation time is shown in Figure 3. All values represent mean values of measurements on three samples.

For the unsealed as well as the PUR- and alkyd-sealed samples, a significant improvement of the colour stability is visible for the thin coated samples (A). Coating systems including a thick coating (B) only exhibit negligible colour changes. Likewise, all samples manifest a darkening by decreasing values for $L$ (cf. Figure 4), with strong changes for the reference samples, slight changes for thin coated samples and insignificant changes for thick coated samples. $\Delta E$ seemed to increase more strongly for PUR-sealed samples; therefore, it was tested for statistical significance. ANOVA, Bonferroni and Tukey-HSD all yielded a significant difference between the evolution of $\Delta E$ for unsealed as well as PUR-sealed samples on a level of $p=0.05$. However, since only three samples were investigated for each coating system, the statistical evaluation may not be entirely reliable. The chromaticity parameters $a$ and $b$ (not shown) also reveal distinct changes (increasing redness and yellowness, respectively) for the coating systems without particles. Changes for samples covered with a thick coating remain negligible while thin coated samples exhibit very little colour changes compared to the uncoated samples. These colour changes of wood exposed to UV light are known to be generated by a decomposition and oxidation of lignin [3,5,34,35], which was observed also in our studies (see IR measurements below). Because of the similar evolution with increasing irradiation time, colour changes of uncoated but sealed samples are assumed to be mainly caused by chemical changes of the underlying wood sample.

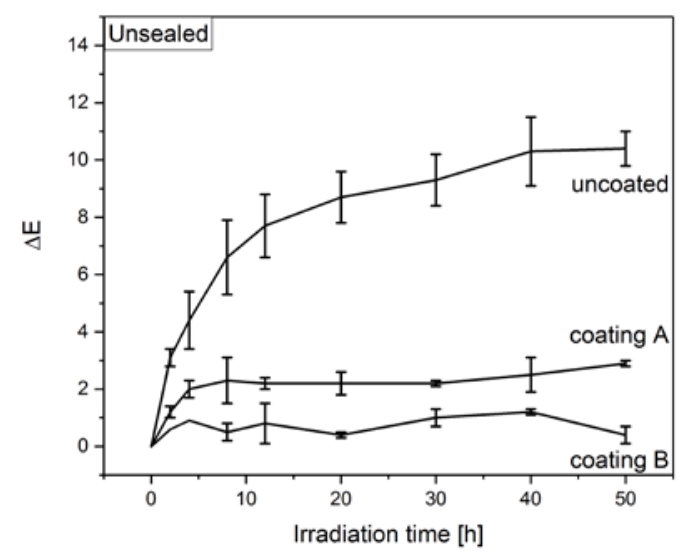

(a)

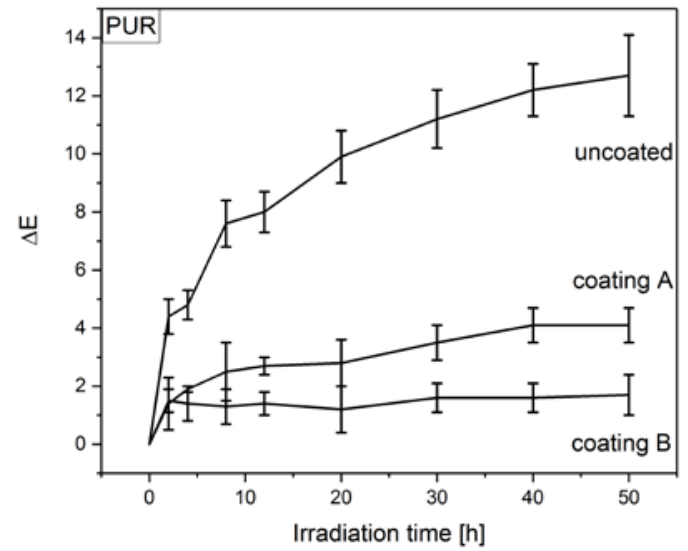

(b)

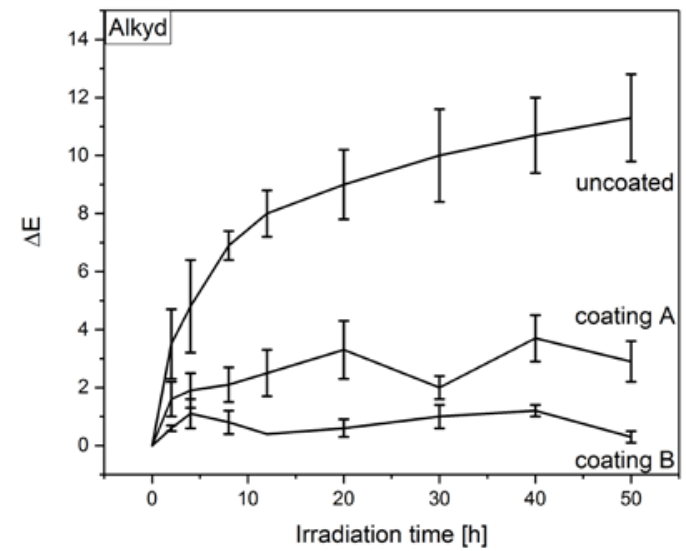

(c)

Figure 3: Variation of total colour change with irradiation time. (a): Unsealed samples, (b): PUR-sealed samples, (c): alkyd-sealed samples. 


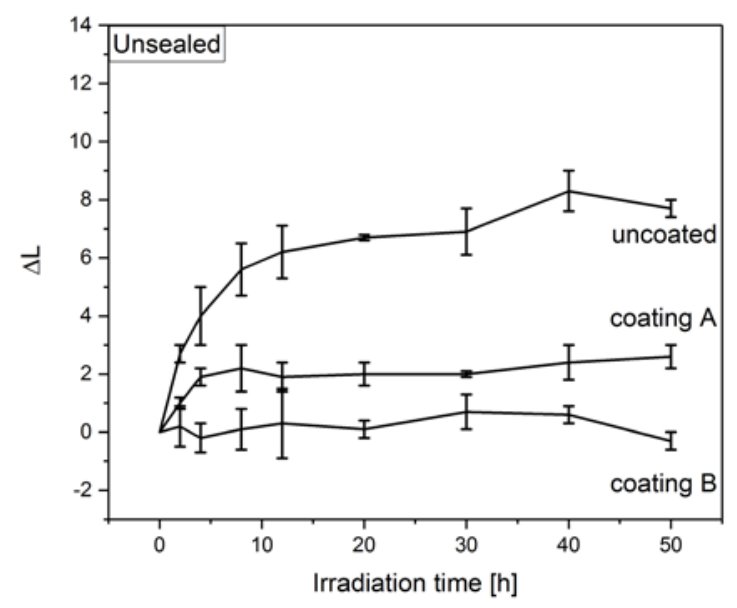

(a)

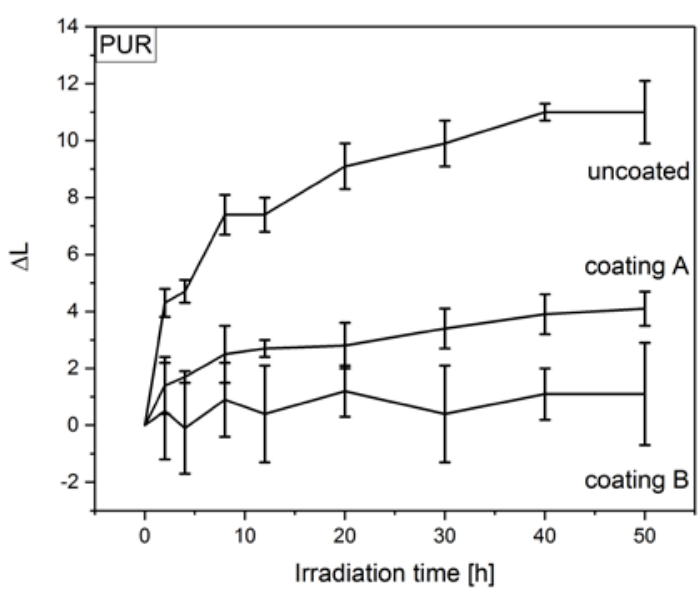

(b)

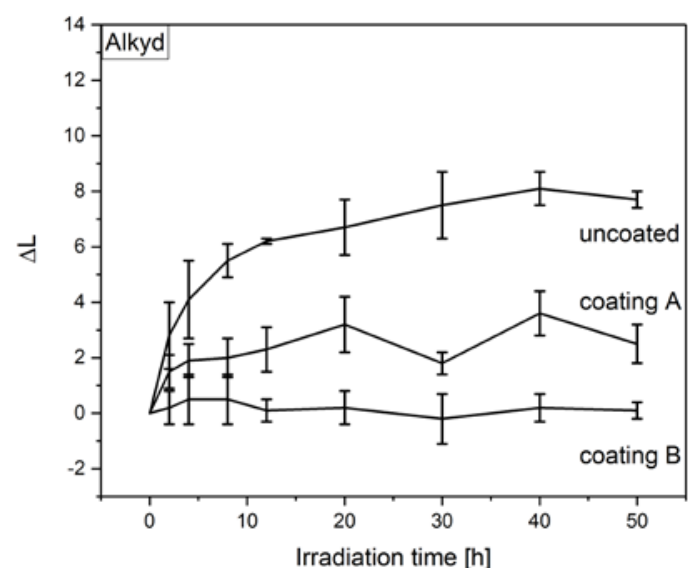

(c)

Figure 4: Variation of the lightness $L$ with irradiation time. (a): Unsealed samples, (b): PUR-sealed samples, (c): alkyd-sealed samples.

\section{Chemical changes induced by UV irradiation}

Figure 5 shows representative IR spectra of uncoated and unsealed wood before and after UV irradiation for 50 hours along with the corresponding difference spectrum. Since the chemical changes are primarily visible in the fingerprint region, only the range from $1850-800 \mathrm{~cm}^{-1}$ is displayed. All spectra were normalised to the highest peak at $1028 \mathrm{~cm}^{-1}$ which features only a minor contribution from lignin [36,37] and mainly results from $\mathrm{C}-\mathrm{O}$ stretching in cellulose and hemicellulose [37-40]. These components are known to be affected less by UV light than lignin [5]. Even though the wood structure was not entirely covered in the case of particle-coated samples, reliable ATR measurements were not possible since the coating prevented a sufficient contact between the wood surface and the ATR crystal. Therefore, only results for uncoated beech wood are discussed in absence of a sealing.

The results in this study coincide with changes reported in the literature [9,38,41-44]. Peaks mainly characteristic for lignin in wood are reduced, e. g. at $1596 \mathrm{~cm}^{-1}(\mathrm{C}=\mathrm{C}$ aromatic skeletal vibrations), and $1464 \mathrm{~cm}^{-1} / 1422 \mathrm{~cm}^{-1}$ (C-H deformation). In particular the decreasing peak at $1503 \mathrm{~cm}^{-1}(\mathrm{C}=\mathrm{C}$ aromatic skeletal vibrations) confirms the degradation of lignin since it does not overlap considerably with peaks resulting from other wood components [45]. On the other hand, photooxidation is manifested in particular through a strong increase and broadening of the peak at $1730 \mathrm{~cm}^{-1}(\mathrm{C}=\mathrm{O}$ stretching vibration), indicating the formation of new and different carbonyl compounds $[38,41,46]$ such as quinones which appear in the region below $1700 \mathrm{~cm}^{-1}$ [34]. The formation of these new chromophoric carbonyl groups is known to correlate well with UV light induced colour changes $[34,35]$. 


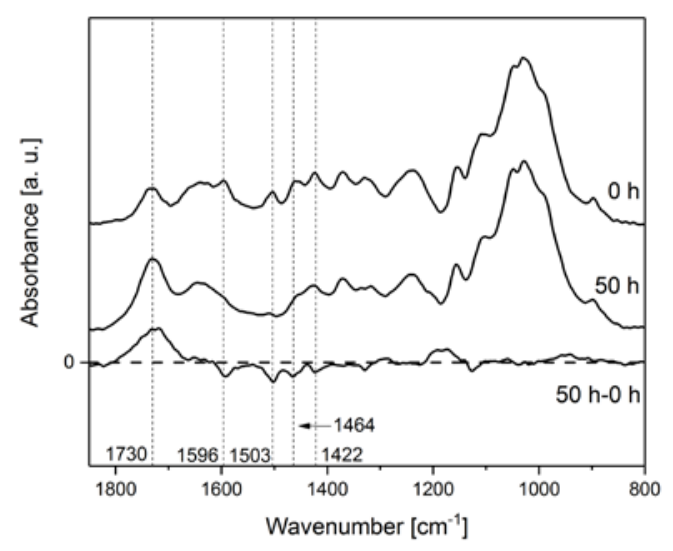

Figure 5: IR fingerprint region of pristine $(0 \mathrm{~h})$ and irradiated $(50 \mathrm{~h})$ beech surfaces along with their diffe re nce spectrum (50 h-0 h).

IR spectra of uncoated but PUR-sealed samples before and after irradiation are displayed in Figure 6a. Band assignment was done based on literature results [47-50]: Around $3700-3200 \mathrm{~cm}^{-1}, \mathrm{OH}$ and NH stretching vibrations are evident. Methyl and methylene absorptions appear around $3000-2800 \mathrm{~cm}^{-1}$ and the carbonyl region is located approx. between $1800-1650 \mathrm{~cm}^{-1}$. The peak at $1530 \mathrm{~cm}^{-1}$ may result from a coupling of $\mathrm{NH}$ bending vibrations with $\mathrm{C}-\mathrm{N}$ stretching vibrations. $\mathrm{C}-\mathrm{O}$ stretching and $\mathrm{C}-\mathrm{H}$ bending vibrations are visible approx. from $1500-1100 \mathrm{~cm}^{-1}$. All spectra were normalised to the highest peak at $1682 \mathrm{~cm}^{-1}(\mathrm{C}=\mathrm{O})$.

Despite the slightly more pronounced colour changes, PUR-coated samples did not exhibit strong chemical changes after $50 \mathrm{~h}$ of UV irradiation. For PUR-sealed and uncoated samples, only minor changes in the methyl/methylene and carbonyl region occurred. The particle coatings seemed to have a stabilising effect on the coating system (cf. Figure 6(b) and 6(c)). Therefore, a coating system composed of a $\mathrm{Zn} / \mathrm{ZnO}$ particle coating and the PUR used in this study appears to be a promising approach to create UV-blocking layers on wooden surfaces.

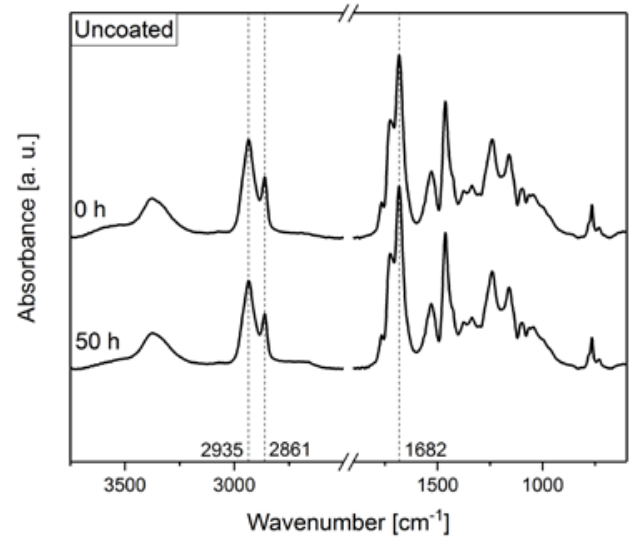

(a)

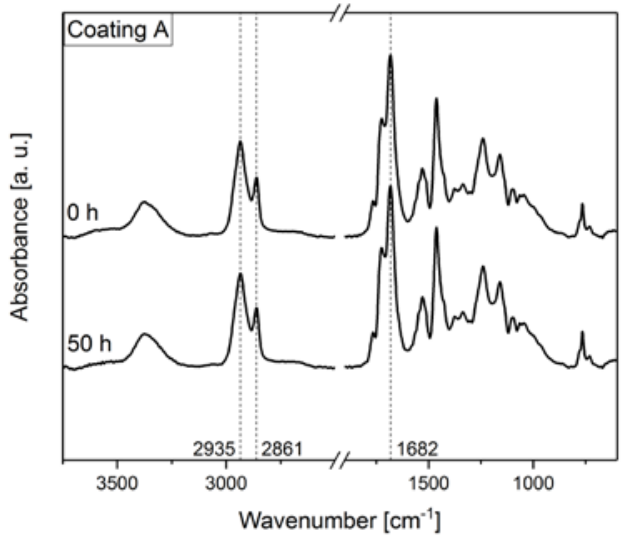

(b) 


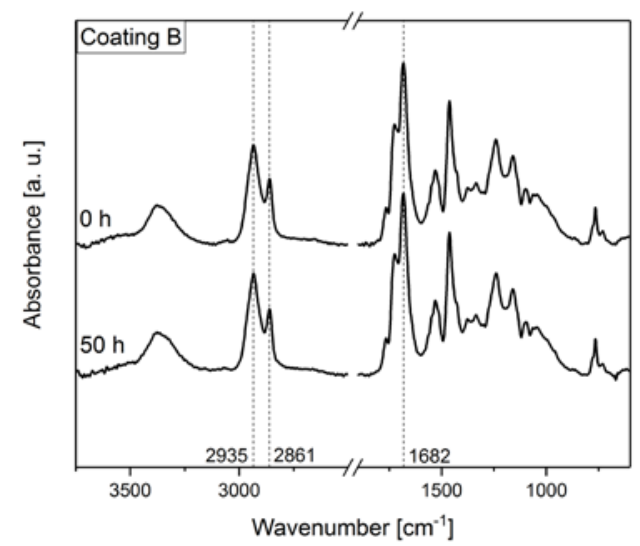

(c)

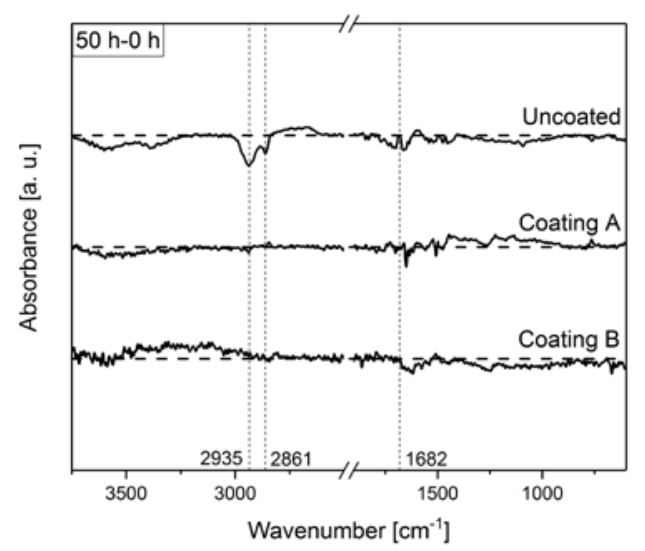

(d)

Figure 6: IR spectra of PUR-sealed samples before and after irradiation. (a): Without particle coating, (b): thin particle coating, (c): thick particle coating, (d): resulting difference spectra for each coating system with dashed horizontal lines denoting zero values.

In contrast, alkyd-sealed samples showed distinct chemical differences before and after UV irradiation. The relevant region in the IR range is depicted in Figure 7 for representative measurements. All spectra were normalised to the highest peak at $1723 \mathrm{~cm}^{-1}$ generated by $\mathrm{C}=\mathrm{O}$ stretching [51-53]; however, this peak was also affected by UV irradiation. Difference spectra are therefore not discussed. Despite this unavoidable inaccuracy, the main changes observed were in agreement with literature results as discussed below.

Without the presence of $\mathrm{Zn} / \mathrm{ZnO}$ particles (cf. Figure 7(a)), main changes were located at $2956 \mathrm{~cm}^{-1}$, $2924 \mathrm{~cm}^{-1}$, and $2853 \mathrm{~cm}^{-1}$ (C-H stretching, decreasing intensity). These changes may be attributed to an ongoing and terminating curing process involving the emission of volatile low molecular components under the influence of UV light as well as degradation processes through Norrish type I and II reactions [51,52]. The curing process in alkyd paints may even continue one month after its application to a surface $[53,54]$. The peak at $1261 \mathrm{~cm}^{-1}$ arises from O-C-O bonds in the polyester part of the alkyd [51,55] and decreased after the ongoing curing process and degradation during UV irradiation. Furthermore, a broadening of the peak at $1723 \mathrm{~cm}^{-1}$ took place, indicating the generation of new carbonyl compounds such as ketones, aldehydes or carboxylic acids [51,52]. The region between approx. $1300-900 \mathrm{~cm}^{-1}$ accounts for $\mathrm{C}-\mathrm{O} / \mathrm{C}-\mathrm{H}$ bonds $[53,56]$ and $\mathrm{OH}$ stretching vibrations are apparent through a broad peak around $3480 \mathrm{~cm}^{-1}$.

The particle coatings appeared to intensify the degradation process, which is assumed to be due to the photocatalytic activity of $\mathrm{ZnO}$ [12-15]. For pigmented alkyd surfaces, the carbonyl peak that was used for normalisation is likely to be affected as well [55]; however, since all other peaks also change [51,55], it was kept as reference because it suffers less from overlaps with other peaks. Therefore, it has to be particularly taken into account that the spectra displayed only represent intensities relative to the $\mathrm{C}=\mathrm{O}$ bond. Contrary to the unpigmented alkyd sealing, especially the $\mathrm{OH}$ peak showed a significant broadening with a new maximum around $3340 \mathrm{~cm}^{-1}$. This peak may be attributed to the formation of carboxylic acids [55,57,58], which indicate an ageing process $[52,58]$ that took place much faster than for the unpigmented paint.

Due to a lack of information about the alkyd's specific composition, precise degradation processes cannot be named. Nevertheless, a couple of general pathways are discussed in the literature. The chemical changes may be partly explained by ongoing curing reactions (e. g. cross-linking, emission of volatile components) manifested for example by a decrease of methyl and methylene absorption [51,52]. The final alkyd film is susceptible to further reactions with radicals. Radicals can be formed through oxidative ageing involving $\beta$-scission, leading for example to the formation of aldehydes, alcohols and carboxylic acids $[59,60]$ which were also visible in the spectra presented in this study. This result is in agreement with the shift of the carbonyl peak to smaller wavelengths. Norrish type I reactions may also lead to the formation of peroxy radicals and induce similar reactions 
as oxidative ageing involving $\beta$-scission [59,60]. Moreover, the carbonyl group may react through Norrish type II processes $[51,59,60]$.

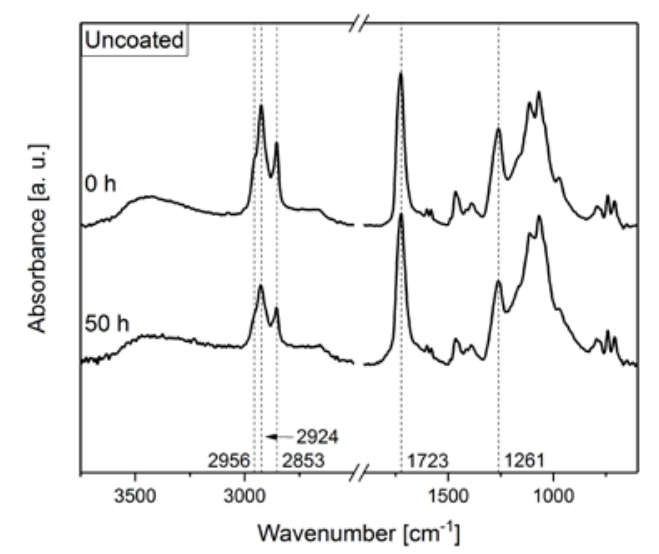

(a)

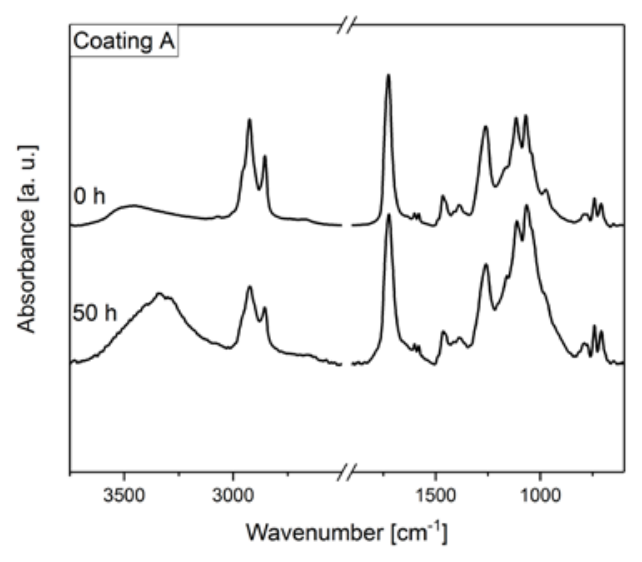

(b)

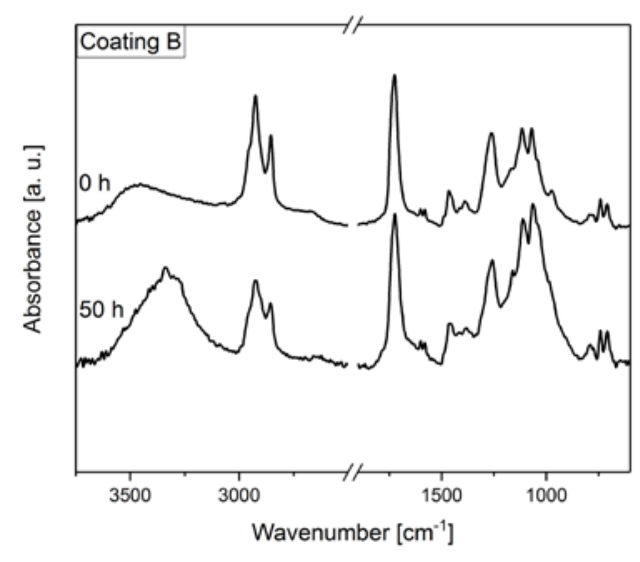

(c)

Figure 7: IR spectra of alkyd sealed samples before and after irradiation. (a): Without particle coating, (b): thin particle coating, (c): thick particle coating.

In summary, the chemical analysis confirmed degradation processes of uncoated and unsealed wood exposed to UV light. Alkyd sealings appeared to be distinctly affected by the irradiation process. Their application to particle-coated samples even led to accelerated ageing caused by photocatalytic degradation, making them unsuitable for a UV protective coating on wooden surfaces. In contrast, PUR-sealed samples without particle coatings exhibited only negligible changes in chemical structure. The prior application of a particle coating even stabilised the coating system and therefore represents a promising candidate for the further development of UV-blocking layers on wood and wood products.

\section{Conclusion and Outlook}

In this study, $\mathrm{Zn} / \mathrm{ZnO}$ coatings deposited by cold plasma spraying on wood and polypropylene were investigated with regard to morphologic aspects as well as their impact on photodegradation of wood and possible sealing materials. SEM measurements confirmed strong changes in the shape of the used particles following the deposition, thus confirming a melting and solidifying process. Moreover, a partial visibility of wood structure was affirmed. Roughness measurements showed a slight roughness increase for particle-coated beech, as measured by the $R k$ parameter, and a tendency of particles to increase the surface peaks as well as to reduce wood anatomical gaps, most probably by filling the wood pores to some extent.

The application of particle coatings significantly improved the colour stability of unsealed and alkydor PUR-sealed specimens. Whereas samples coated with concentration A still underwent minor 
discolouration upon UV irradiation, those coated with concentration B did not change. This behaviour was observed both for unsealed as well as for sealed beech wood samples, indicating that photocatalytic degradation of the matrix material by $\mathrm{ZnO}$, though it did occur for alkyd-sealed samples, did not affect the colour within $50 \mathrm{~h}$ of UV irradiation.

Despite negligible discolouration, chemical analysis showed distinct changes for alkyd-sealed samples. Strong changes in the carbonyl region in addition to modifications in the $\mathrm{C}-\mathrm{O} / \mathrm{C}-\mathrm{H}$ structure along with the appearance of $\mathrm{OH}$ bonds in carboxylic acids indicated photocatalytic degradation processes.

In contrast, the PUR matrix did not seem to be much affected by UV irradiation. Slight changes did occur to reference samples without particle coatings, but the application of $\mathrm{Zn} / \mathrm{ZnO}$ coatings seemed to have stabilised the coating system.

Particularly the coating system including a $\mathrm{Zn} / \mathrm{ZnO}$ particle coating along with the PUR sealing represents a promising approach to creating UV protective layers on wood in a relatively easy way. The used plasma setup operates at atmospheric pressure, requires relatively short treatment times and is suitable for coating thermosensitive materials like wood. Hence, the application of such a particle coating followed by a conventional sealing may be suitable for in-line processing. The sequential application of particles followed by a PUR sealing permits a precise dosage of UV-blocking filters and matrix material. Nanoparticle release due, for example, to leaching or abrasion is expected to be inhibited by such a coating system, but needs to be investigated in separate studies. Moreover, since $\mathrm{ZnO}$ features biocidal properties, the presented coating systems could therefore be tested as protective layers against bacteria and fungi.

\section{Acknowledgements}

Financial support from the Lower Saxony Ministry of Science and Culture is gratefully acknowledged. The authors further thank Max Baumung, Ghiath Jnido, Monika Gelker and Robert Koslowski for technical assistance, Roger Skarsten for proofreading the manuscript and ECKART GmbH for providing the used powder material. Moreover, the authors hereby acknowledge the structural funds project PRO-DD (POS-CCE, O.2.2.1., ID 123, SMIS 2637, ctr. No. 11/2009) for providing the infrastructure used in this work for surface roughness measurements.

\section{Niedersächsisches Ministerium für Wissenschaft und Kultur}

\section{References}

[1] M. Shimada and M. Takahashi, "Biodegradation of cellulosic materials," in Wood and Cellulosic Chemistry, D. N.-S. Hon and N. Shiraishi, Eds., Marcel Dekker, New York, 1991.

[2] T. K. Kirk and E. B. Cowling, "Biological decomposition of solid wood," in The Chemistry of Solid Wood, R. Rowell, Ed., vol. 207, pp. 455-487, American Chemical Society, 1984.

[3] W. C. Feist and D. N.-S. Hon, "Chemistry of weathering and protection," in The Chemistry of Solid Wood, R. Rowell, Ed., vol. 207, pp. 401-451, American Chemical Society, 1984.

[4] B. George, E. Suttie, A. Merlin et al., "Photodegradation and photostabilisation of wood - the state of the art," Polymer Degradation and Stability, vol. 88, no. 2, pp. 268-274, 2005.

[5] D. N.-S. Hon, "Photochemistry of wood," in Wood and Cellulosic Chemistry, D. N.-S. Hon and N. Shiraishi, Eds., Marcel Dekker, New York, 1991.

[6] C. A. Clausen, F. Green, and S. Nami Kartal, "Weatherability and leach resistance of wood impregnated with nano-zinc oxide," Nanoscale Research Letters, vol. 5, no. 9, pp. 1464-1467, 2010.

[7] Y. Yu, Z. Jiang, G. Wang et al., "Growth of $\mathrm{ZnO}$ nanofilms on wood with improved photostability," Holzforschung, vol. 64, no. 3, pp. 385-390, 2010.

[8] S. M. Fufa, B. P. Jelle, and P. J. Hovde, "Effects of $\mathrm{TiO}_{2}$ and clay nanoparticles loading on weathering performance of coated wood," Progress in Organic Coatings, vol. 76, no. 10, pp. 1425-1429, 2013. 
[9] J. Salla, K. K. Pandey, and K. Srinivas, "Improvement of UV resistance of wood surfaces by using ZnO nanoparticles," Polymer Degradation and Stability, vol. 97, no. 4, pp. 592-596, 2012.

[10] P. H. M. Hoet, I. Brüske-Hohlfeld, and O. V. Salata, "Nanoparticles - known and unknown health risks," Journal of Nanobiotechnology, vol. 2, no. 1, p. 12, 2004.

[11] A. Baun, N. B. Hartmann, K. Grieger et al., "Ecotoxicity of engineered nanoparticles to aquatic invertebrates: a brief review and recommendations for future toxicity testing," Ecotoxicology, vol. 17 , no. 5, pp. 387-395, 2008.

[12] A. Rezaee, H. Rangkooy, A. Khavanin et al., "High photocatalytic decomposition of the air pollutant formaldehyde using nano- $\mathrm{ZnO}$ on bone char," Environmental Chemistry Letters, vol. 12, no. 2, pp. 353-357, 2014.

[13] H. F. Moafi, A. F. Shojaie, and M. A. Zanjanchi, "Photocatalytic self-cleaning properties of cellulosic fibers modified by nano-sized zinc oxide," Thin Solid Films, vol. 519, no. 11, pp. 3641-3646, 2011.

[14] C. A. Gouvêa, F. Wypych, S. G. Moraes et al., "Semiconductor-assisted photocatalytic degradation of reactive dyes in aqueous solution," Chemosphere, vol. 40, no. 4, pp. 433-440, 2000.

[15] A. McLaren, T. Valdes-Solis, G. Li et al., "Shape and size effects of $\mathrm{ZnO}$ nanocrystals on photocatalytic activity," Journal of the American Chemical Society, vol. 131, no. 35, pp. 1254012541, 2009.

[16] W. F. Sullivan, "Weatherability of titanium-dioxide-containing paints," Progress in Organic Coatings, vol. 1, no. 2, pp. 157-203, 1972.

[17] M. Bente, G. Avramidis, S. Förster et al., "Wood surface modification in dielectric barrier discharges at atmospheric pressure for creating water repellent characteristics," Holz als Rohund Werkstoff, vol. 62, no. 3, pp. 157-163, 2004.

[18] J. Profili, O. Levasseur, A. Koronai et al., "Deposition of nanocomposite coatings on wood using cold discharges at atmospheric pressure," Surface and Coatings Technology, vol. 309, pp. 729737, 2017.

[19] G. Toriz, M. G. Gutiérrez, V. González-Alvarez et al., "Highly hydrophobic wood surfaces prepared by treatment with atmospheric pressure dielectric barrier discharges," Journal of Adhesion Science and Technology, vol. 22, no. 16, pp. 2059-2078, 2008.

[20] S. Zanini, C. Riccardi, M. Orlandi et al., "Wood coated with plasma-polymer for water repellence," Wood Science and Technology, vol. 42, no. 2, pp. 149-160, 2008.

[21] P. Gascón-Garrido, N. Mainusch, H. Militz et al., "Copper and aluminium deposition by coldplasma spray on wood surfaces: effects on natural weathering behaviour," European Journal of Wood and Wood Products, vol. 75, no. 3, pp. 315-324, 2016.

[22] P. Gascón-Garrido, N. Mainusch, H. Militz et al., "Effects of copper-plasma deposition on weathering properties of wood surfaces," Applied Surface Science, vol. 366, pp. 112-119, 2016.

[23] L. M. Wallenhorst, S. Dahle, M. Vovk et al., "Characterisation of PMMA/ATH layers realised by means of atmospheric pressure plasma powder deposition," Advances in Condensed Matter Physics, vol. 2015, no. 3, pp. 1-12, 2015.

[24] L. M. Wallenhorst, L. Loewenthal, G. Avramidis et al., "Topographic, optical and chemical properties of zinc particle coatings deposited by means of atmospheric pressure plasma," Applied Surface Science, vol. 410, pp. 485-493, 2017.

[25] L. Gurău, The roughness of sanded wood surfaces, Dissertation, Brunel University, 2004.

[26] International Organization for Standardization, "Geometrical product specification (GPS) Filtration - Part 31: Robust profile filters: Gaussian regression filters.," ISO/TS 16610-31:2010.

[27] L. Gurău, H. Mansfield-Williams, and M. Irle, "Filtering the roughness of a sanded wood surface," Holz als Roh- und Werkstoff, vol. 64, no. 5, pp. 363-371, 2006.

[28] P. L. Tan, S. Sharif, and I. Sudin, "Roughness models for sanded wood surfaces," Wood Science and Technology, vol. 46, no. 1, pp. 129-142, 2012.

[29] International Organization for Standardization, "Geometrical product specifications (GPS). Surface texture: Profile method-Terms, definitions and surface texture parameters.," ISO 4287:1997 + Amd1: 2009.

[30] International Organization for Standardization, "Geometrical product specifications (GPS) Surface texture: Profile method. Surfaces having stratified functional properties. Part 2: Height characterisation using the linear material ratio curve.," ISO 13565-2:1996 + Cor 1:1998. 
[31] E. Westkamper and A. Riegel, "Qualitätskriterien für geschliffene Massivholzoberflächen," Holz als Roh- und Werkstoff, vol. 51, no. 2, pp. 121-125, 1993.

[32] "Paints and varnishes - Coating materials and coating systems for exterior wood - Part 6: Exposure of wood coatings to artificial weathering using fluorescent UV lamps and water," DIN EN 927-6:2016.

[33] L. Gurău, C. Csiha, and H. Mansfield-Williams, "Processing roughness of sanded beech surfaces," European Journal of Wood and Wood Products, vol. 73, no. 3, pp. 395-398, 2015.

[34] U. Müller, M. Rätzsch, M. Schwanninger et al., "Yellowing and IR-changes of spruce wood as result of UV-irradiation," Journal of Photochemistry and Photobiology B: Biology, vol. 69, no. 2, pp. 97-105, 2003.

[35] K. K. Pandey, "Study of the effect of photo-irradiation on the surface chemistry of wood," Polymer Degradation and Stability, vol. 90, no. 1, pp. 9-20, 2005.

[36] O. Faix, "Fourier Transform Infrared Spectroscopy," in Lin, Dence (Ed.) - Methods in Lignin Chemistry, pp. 83-109.

[37] P. Verma, U. Junga, H. Militz et al., "Protection mechanisms of DMDHEU treated wood against white and brown rot fungi," Holzforschung, vol. 63, no. 3, 2009.

[38] C.-M. Popescu, M.-C. Popescu, and C. Vasile, "Structural analysis of photodegraded lime wood by means of FT-IR and 2D IR correlation spectroscopy," International Journal of Biological Macromolecules, vol. 48, no. 4, pp. 667-675, 2011.

[39] H. G. Higgins, C. M. Stewart, and K. J. Harrington, "Infrared spectra of cellulose and related polysaccharides," Journal of Polymer Science, vol. 51, no. 155, pp. 59-84, 1961.

[40] B. Mohebby, "Application of ATR infrared spectroscopy in wood acetylation," Journal of Agricultural Science and Technology, vol. 10, no. 3, pp. 253-259, 2008.

[41] L. Calienno, A. Lo Monaco, C. Pelosi et al., "Colour and chemical changes on photodegraded beech wood with or without red heartwood," Wood Science and Technology, vol. 48, no. 6, pp. 1167-1180, 2014.

[42] M. C. Timar, A. M. Varodi, and L. Gurău, "Comparative study of photodegradation of six wood species after short-time UV exposure," Wood Science and Technology, vol. 50, no. 1, pp. 135163, 2016.

[43] L. Tolvaj and O. Faix, "Artificial Ageing of Wood Monitored by DRIFT Spectroscopy and CIE L*a*b* Color Measurements. 1. Effect of UV Light," Holzforschung, vol. 49, no. 5, pp. 397-404, 1995.

[44] L. Tolvaj, C.-M. Popescu, Z. Molnar et al., "Effects of air relative humidity and temperature on photodegradation processes in beech and spruce wood," BioResources, no. 11, 2016.

[45] K. K. Pandey, "A study of chemical structure of soft and hardwood and wood polymers by FTIR spectroscopy," Journal of Applied Polymer Science, vol. 71, no. 12, pp. 1969-1975, 1999.

[46] D. N.-S. Hon and S.-T. Chang, "Surface degradation of wood by ultraviolet light," Journal of Polymer Science: Polymer Chemistry Edition, vol. 22, no. 9, pp. 2227-2241, 1984.

[47] H. Wang, Y. Wang, D. Liu et al., "Effects of additives on weather-resistance properties of polyurethane films exposed to ultraviolet radiation and ozone atmosphere," Journal of Nanomaterials, vol. 2014, no. 4, pp. 1-7, 2014.

[48] H. Kim and M. W. Urban, "Molecular level chain scission mechanisms of epoxy and urethane polymeric films exposed to $\mathrm{UV} / \mathrm{H}_{2} \mathrm{O}$. Multidimensional spectroscopic studies," Langmuir, vol. 16, no. 12, pp. 5382-5390, 2000.

[49] D. Rosu, L. Rosu, and C. N. Cascaval, "IR-change and yellowing of polyurethane as a result of UV irradiation," Polymer Degradation and Stability, vol. 94, no. 4, pp. 591-596, 2009.

[50] X. Yang, C. Vang, D. Tallman et al., "Weathering degradation of a polyurethane coating," Polymer Degradation and Stability, vol. 74, no. 2, pp. 341-351, 2001.

[51] F. X. Perrin, M. Irigoyen, E. Aragon et al., "Artificial aging of acrylurethane and alkyd paints: A micro-ATR spectroscopic study," Polymer Degradation and Stability, vol. 70, no. 3, pp. 469475, 2000.

[52] V. Pintus, S. Wei, and M. Schreiner, "Accelerated UV ageing studies of acrylic, alkyd, and polyvinyl acetate paints: Influence of inorganic pigments," Microchemical Journal, vol. 124, pp. 949-961, 2016. 
[53] E. M. Salazar-Rojas and M. W. Urban, "Curing of non-pigmented alkyd coatings detected by insitu photoacoustic fourier transform infrared spectroscopy (PA FT-IR)," Progress in Organic Coatings, vol. 16, no. 4, pp. 371-386, 1989.

[54] M. W. Urban and E. M. Salazar-Rojas, "Probing organic-inorganic interactions and curing processes in coatings by photoacoustic fourier transform infrared spectroscopy," Journal of Polymer Science Part A: Polymer Chemistry, vol. 28, no. 6, pp. 1593-1613, 1990.

[55] M. Anghelone, D. Jembrih-Simbürger, and M. Schreiner, "Influence of phthalocyanine pigments on the photo-degradation of alkyd artists' paints under different conditions of artificial solar radiation," Polymer Degradation and Stability, vol. 134, pp. 157-168, 2016.

[56] J. van der Weerd, A. van Loon, and J. J. Boon, "FTIR Studies of the Effects of Pigments on the Aging of Oil," Studies in Conservation, vol. 50, no. 1, pp. 3-22, 2005.

[57] G. Socrates, Infrared and Raman Characteristic Group Frequencies: Tables and Charts, John Wiley \& Sons, Chichester, 2001.

[58] R. Ploeger, D. Scalarone, and O. Chiantore, "The characterization of commercial artists' alkyd paints," Journal of Cultural Heritage, vol. 9, no. 4, pp. 412-419, 2008.

[59] S. M. Cakić, I. S. Ristić, J. M. Vladislav et al., "IR-change and colour changes of long-oil air drying alkyd paints as a result of UV irradiation," Progress in Organic Coatings, vol. 73, no. 4, pp. 401-408, 2012.

[60] J. Mallégol, J.-L. Gardette, and J. Lemaire, "Long-term behavior of oil-based varnishes and paints. Photo- and thermooxidation of cured linseed oil," Journal of the American Oil Chemists' Society, vol. 77, no. 3, pp. 257-263, 2000. 


\title{
Paper III
}

\section{Characterisation of PMMA/ATH Layers realised by means of Atmospheric Pressure Plasma Powder Deposition}

\author{
Lena M. Wallenhorst ${ }^{1}$, Sebastian Dahle ${ }^{2,3}$, Matej Vovk ${ }^{4,5}$, Lisa Wurlitzer ${ }^{2,3}$, \\ Leander Loewenthal $^{1}$, Nils Mainusch ${ }^{6}$, Christoph Gerhard ${ }^{6}$, Wolfgang Viöl ${ }^{1,6}$
}

${ }^{1}$ University of Applied Sciences and Arts, Laboratory of Laser and Plasma Technologies, Von-Ossietzky-Straße 99, 37085 Göttingen, Germany

${ }^{2}$ Clausthal University of Technology, Institute of Energy Research and Physical Technologies, Leibnizstraße 4, 38678 Clausthal-Zellerfeld, Germany

${ }^{3}$ Clausthal University of Technology, Clausthal Center of Material Technology (CZM), Agricolastraße 2, 38678 Clausthal-Zellerfeld, Germany

${ }^{4}$ University of Ljubljana, Biotechnical Faculty, Jamnikarjeva 101, 1000 Ljubljana, Slovenia

${ }^{5}$ Kolpa d. d., Rosalnice 5, 8330 Metlika, Slovenia

${ }^{6}$ Fraunhofer Institute for Surface Engineering and Thin Films, Application Center for Plasma and Photonics, Von-Ossietzky-Straße 100, 37085 Göttingen, Germany

\begin{abstract}
We report on the characteristics of aluminium trihydrate filled poly(methyl methacrylate) composite (PMMA/ATH) coatings realised by plasma deposition at atmospheric pressure. For this purpose, PMMA/ATH powder was fed to a plasma jet where the process and carrier gas was compressed air. The deposited coatings were investigated by X-ray photoelectron spectroscopy and water contact angle measurements. Further, the raw material was characterised before deposition. It was found that, with respect to the raw material, aluminium was uncovered in the course of the plasma deposition process which can be explained by plasma-induced etching of the PMMA matrix. As a result, the wettability of plasma-deposited PMMA/ATH was significantly increased. Even though a uniform coating film could not be realised as ascertained by confocal laser scanning microscopy, the deposited coatings feature notably enhanced characteristics which could be advantageous for further processing.
\end{abstract}

\section{Introduction}

The application of inorganic fillers as reinforcement within polymer composites is a well-known approach [1] that is still commonly applied [2]. One particular composite material that has been continuously improved in the past decades and is still having an increasing number of applications is poly(methyl methacrylate) (PMMA) reinforced with aluminium trihydrate $\left(\mathrm{Al}(\mathrm{OH})_{3}, \mathrm{ATH}\right)$. The PMMA/ATH composite is classified as solid material[3] and was developed and described by Duggins and coworkers [4,5]. Several different compositions and fabrication processes are commonly in use, but in general PMMA/ATH composites consist of about $40 \%$ PMMA and $60 \%$ ATH. All composites of this kind feature high moisture resistance, good chemical resistance, and UV resistance. They are safe for food contact, easy to clean, and thermoformable [6,7]. The ATH used as inorganic filler within the composite lowers the costs for the fabrication of this material and further drastically improves the mechanical properties such as elastic modulus [8], abrasion, and wear resistance [9] as well as the fatigue life [10].The ATH also acts as a flame retardant and smoke suppressant [11-13].

For many applications, ATH filler particles with average sizes of approximately $2-10 \mu \mathrm{m}$ are used, which typically occur as agglomerates of $40-50 \mu \mathrm{m}$ [14]. Otherwise, bead milling can be used to 
ensure an average filler particle size below $1 \mu \mathrm{m}$, consequently improving the distribution of the filler particles within the matrix [15]. Furthermore, silane coupling agents [16] are usually applied to ensure an excellent dispersion [15] and enhance the mechanical properties $[9,10]$ as well as the thermal stability of the composite material [15].

However, the final processing of PMMA/ATH composite materials may be complicated. For example, it has been found to be quite challenging to form a structural joint via bonding of PMMA/ATH material with wood and wood composites [17]. Another open issue is the recycling of PMMA/ATH composite materials. The shaping and polishing of PMMA/ATH work pieces produce an estimated amount of 1200 tons of PMMA/ATH waste dust per year only in Slovenia [18]. Even though the material itself exhibits outstanding mechanical and chemical properties, residual powder and chips can hardly be used in an industrial and economically viable way. Kaminsky and colleagues [19] have carried out the pyrolysis of a PMMA/ATH composite. Pyrolysis of PMMA is already an established way of obtaining methyl methacrylate monomer (MMA), because at $450^{\circ} \mathrm{C}$ as much as $97.2 \%$ of MMA can be obtained. Authors investigated if it is possible to obtain similarly high proportions of MMA from PMMA/ATH composites with high content of ATH and whether there is a catalytic effect of ATH in the process of pyrolysis. The results showed that the pyrolysis of the PMMA/ATH obtained only $58 \%$ of the MMA, which is a much smaller percentage compared to $97 \%$, which was obtained from pure PMMA. By-products of the thermal decomposition of this composite were the hydrolysis products of MMA, such as methacrylic acid, methanol, and isobutyl acid. By lowering the temperature to $400^{\circ} \mathrm{C}$, they have managed to increase the obtained MMA to $65 \%$ by weight. They found that such a low proportion of acquired MMA is a consequence of the water released from the pyrolysis of ATH and chemical stabilizers in the composite. The high proportion of aluminium components practically has no catalytic effect on hydrolysis, because they got the same results by using the water vapour as fluidising medium instead of nitrogen [19]. However, some innovative approaches recently try to make use of the waste powder, for example, as filler for bitumen [18].

In a recent approach, the use of a dielectric barrier discharge (DBD) plasma for the modification of PMMA/ATH work pieces has been evaluated, that is, trying to functionalise the PMMA/ATH surface and to improve the bonding of glues and other polymers to the modified PMMA/ATH surface. In the literature, several effects of cold plasma discharges on PMMA are reported, namely,

(a) an oxidation of the polymer [20],

(b) a homogeneous etching of the polymer [21],

(c) a generation of micro- or nanopatterns on the polymer's surface [22].

All of these effects could be promising for an enhancement of the bonding strength upon gluing as well as for the use of the processed powder as additive to polymer resins. The oxidation of the polymer (a) should happen by the attachment of polar groups, which could then probably act as chemical interlinks between binder and substrate or between composite powder and polymer matrix. The homogeneous etching (b) could lead to exposed ATH particles, allowing an improved attachment of an adhesive or a polymer matrix to the ATH's hydroxyl groups. Thus, these partially exposed filler particles would presumably act as mechanical interlocking and most likely lead to an improved bonding strength via form closure. The inhomogeneous etching (c), that is, the formation of rough micro- and nanostructures, should at least lead to a mechanical interlocking between adhesive and substrate or between composite powder and polymer matrix. However, these expectations were not fulfilled by a DBD pretreatment of PMMA/ATH work pieces. Even though some carboxy groups at the outermost surface layer of the PMMA as well as an increased surface roughness did remarkably reduce the water contact angles, no significant impact on bonding strengths with a water-based adhesive was observed. Due to formation of debris on the surface, representing the weakest link, an increase in bonding strength was prevented. The stability of the bonds against humidity was significantly improved, but still this does not yield any new possibilities for the use of the PMMA/ATH waste powder.

Following these previous results, the results of a plasma treatment of the waste powder using a gliding arc discharge are presented in this contribution. The gas temperatures of gliding arc discharges are usually in the order of magnitude of $1000 \mathrm{~K}$ and above [23], while the increase of sample temperatures during DBD plasma treatments usually would not exceed $10 \mathrm{~K}$ [24]. Correspondingly, the etching rates 
for the polymer are expected to be significantly higher in comparison to the previous approach. As a result, the formation of significant amounts of debris might be inhibited. Furthermore, the presented approach employs remote plasma to directly process the waste powder within a gas stream and concurrently deposit the powder onto a surface.

Remote plasma devices, that is, plasma jets, already have been employed to deposit films onto PMMA substrates via plasma-enhanced chemical vapour deposition [25]. Furthermore, the use of remote plasma devices for the functionalization of aerosol particles has been demonstrated before, for example, for polyethylene particles [26]. The approach to use remote plasma for both a functionalization of aerosol particles and the concurrent deposition of these particles as a functional coating, however, has not yet been reported in literature.

\section{Characterisation of the used PMMAATH powder}

In this work, aluminium trihydrate-reinforced poly(methyl methacrylate) powder from Kolpa, d. d., Slovenia, was used for the realisation of PMMA/ATH layers by atmospheric pressure plasma powder deposition. The mechanical properties of original PMMA/ATH composite boards were already investigated by other groups, yielding a density $\rho$ of $1740 \mathrm{~kg} / \mathrm{m}^{3}$ and a filler particle fraction of 50 wt. \% ATH [18], as well as a modulus of elasticity $E$ of approximately $9700 \mathrm{~N} / \mathrm{mm}^{2}$ and a bending strength $S$ of approximately $70 \mathrm{~N} / \mathrm{mm}^{2}$ [17]. In addition, the constant wetting rate angle (CWRA), the surface energy, the particle size distribution, and the specific surface areas as well as the glass transition temperature were determined in the present work prior to plasma deposition.

\section{Determination of the constant wetting rate angle (CWRA) and surface energy}

For the determination of the constant wetting rate angles of distilled water and diiodomethane drops on the used PMMA/ATH powder, a Krüss Drop Shape Analyser, DSA100, was used. Prior to applying the drops on the samples, the powders were pressed using a hand press Graseby from Specac. In this vein, circular plates with a diameter of $13 \mathrm{~mm}$ and a mass of $275 \pm 5 \mathrm{mg}$ were produced where the pressing time was $10 \mathrm{~s}$ and the pressing pressure was $739.46 \mathrm{~N} / \mathrm{mm}^{2}$. The volume of the applied drops was $1-2 \mu \mathrm{L}$. The process of applying the drops on the substrates was recorded and each drop was subsequently analysed by a circle fitting method from which the contact angle was calculated. Subsequently, the surface energies were determined using the measuring system's Drop Shape Analyser and an implemented evaluation algorithm according to Owens, Wendt, Rabel, and Kaelble (OWRK) [27], which is based on Young's equation, given by

$$
\gamma_{S G}=\gamma_{S L}+\gamma_{L G} \cos \theta .
$$

Here, $\gamma_{\mathrm{SG}}$ is the interfacial energy between solid and gas, $\gamma_{\mathrm{LG}}$ between liquid and gas, and $\gamma_{\mathrm{SL}}$ between solid and liquid. According to the OWRK method, the total energy $\gamma^{\text {tot }}$ is the sum of polar $\left(\gamma^{P}\right)$ and disperse $\left(\gamma^{D}\right)$ fraction. Distilled water $\left(\gamma^{\text {tot }}=72.8 \mathrm{mNm}^{-1}, \gamma^{P}=51 \mathrm{mNm}^{-1}\right.$, and $\gamma^{D}=21.8 \mathrm{mNm}^{-1}$ at $\left.T=20^{\circ} \mathrm{C}\right)$ and diiodomethane $\left(\gamma^{\text {tot }}=50.8 \mathrm{mNm}^{-1}, \gamma^{P}=0 \mathrm{mNm}^{-1}\right.$, and $\gamma^{D}=50.8 \mathrm{mNm}^{-1}$ at $\left.T=20^{\circ} \mathrm{C}\right)$ were used to analyse the constant wetting rate angle on the pressed PMMA/ATH powder pellets and to determine its surface energy. The angles were determined after a transition time of $3 \mathrm{~s}$ after application of the drops since it was visually established that this time interval coincides with the transition from the spreading stage to a constant penetration regime, basically following [28]. All measurements were performed at an environmental humidity of $35 \pm 2 \%$ and a temperature of $22 \pm 1{ }^{\circ} \mathrm{C}$. By this procedure, a constant wetting rate angle of $66.94^{\circ} \pm 0.95^{\circ}$ for distilled water was measured, where the overall surface energy was $49.23 \mathrm{mNm}^{-1}$, given by the sum of the disperse fraction of $40.65 \mathrm{mNm}^{-1}$ and the polar fraction of $8.57 \mathrm{mNm}^{-1}$.

\section{Determination of the particle size distribution and specific surface areas}

The particle size distribution of the powder was determined by laser light scattering with the aid of a particle analyser Mastersizer S from Malvern Instruments. A 300RF lens with a QS small volume sample dispersion unit for measuring liquid dispersion was used. The obscuration was adjusted to $12-$ $15 \%$. Powder samples were dispersed in distilled water using an ultrasonic probe. The dispersion was sonicated for 3 minutes prior to the measurement. Particle size distributions typically include $(0.1),(0.5)$, and $D_{(0.9)}$, which represent the percentages of particles (i.e., $10 \%, 50 \%$, and $90 \%$, resp.) below the given size in microns. By applying the above-described procedure, the particle size distribution was determined to $_{(0.1)}=7.23 \mu \mathrm{m},(0.5)=75.66 \mu \mathrm{m}, D_{(0.9)}=167.82 \mu \mathrm{m}$, and $D_{(\max )}=301.68 \mu \mathrm{m}$. 
The specific surface areas of PMMA/ATH powders were determined via Brunauer-Emmet-Teller (BET) nitrogen adsorption at $77 \mathrm{~K}$ from the adsorption data in the relative pressure interval from $p / p_{0}$ $=0.05$ to 0.3 . For this measurement, a nitrogen adsorption analyser Tristar 3000 from Micromeritics Instruments was used. Prior to the measurements, between 150 and $250 \mathrm{mg}$ of the sample were accurately weighed into sample tubes and degassed overnight at $50^{\circ} \mathrm{C}$ in a vacuum dryer VacPrep 061 from Micromeritics Corporation. In the course of this drying process, the temperature was set to $50^{\circ} \mathrm{C}$ in order to avoid possible thermochemical reactions of the samples. Two replicate measurements were carried out to evaluate the reproducibility. As a result, a BET specific surface of $2.1452 \pm 0.0250 \mathrm{~m}^{2} / \mathrm{g}$ was determined.

\section{Determination of the glass transition temperature}

The glass transition temperature of the used polymer composite powder was determined by differential scanning calorimetry (DSC). A commercial setup DSC 820 from Mettler-Toledo International Incorporation was used to measure in steps of $10 \mathrm{~K}$ within a temperature range from $-10^{\circ} \mathrm{C}$ to $250^{\circ} \mathrm{C}$. During the measurement, technical nitrogen was used as cover gas. To minimise the influence of impurities and residuals, two heating curves and a cooling curve were recorded sequentially, while only the second heating curve was used for the analysis.

The measured DSC curves are shown in Figure 1, representing the curves of both heating cycles as well as the cooling process in between. A detailed view of the relevant second heating curve is given in Figure 1(b), depicting a glass transition temperature $T_{g}$ of about $120^{\circ} \mathrm{C}$.

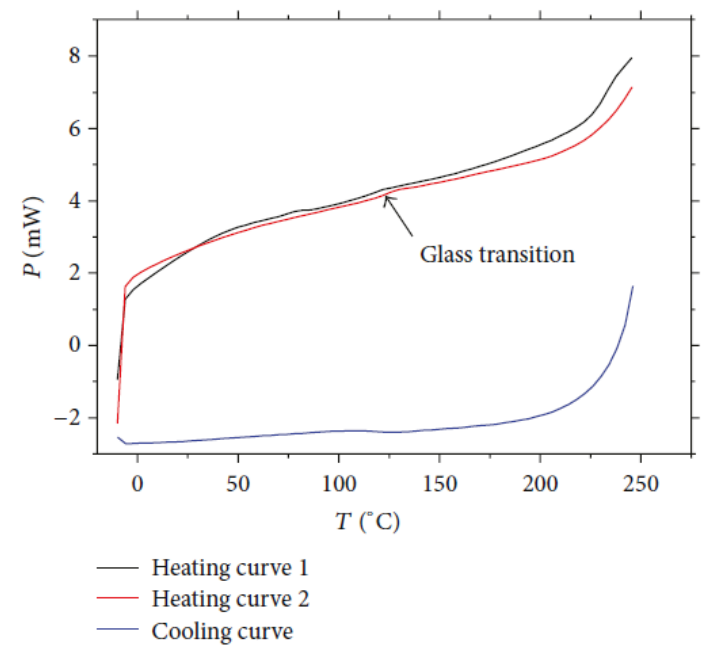

(a)

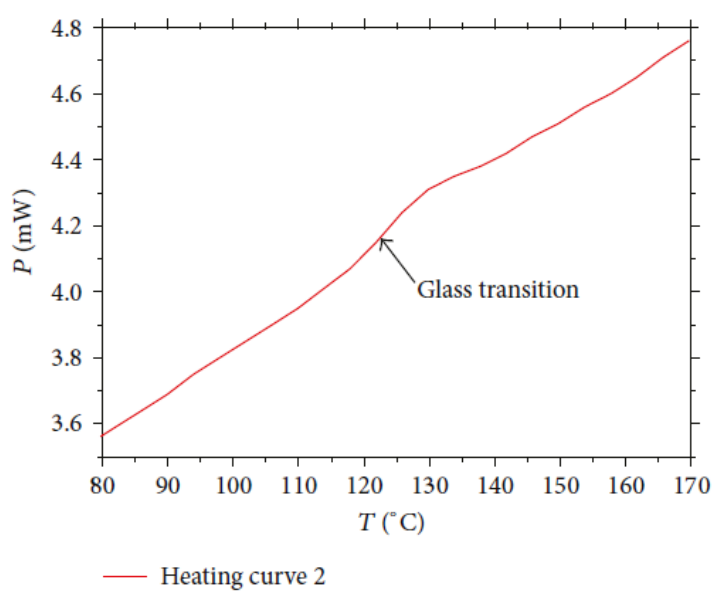

(b)

Figure 1: DSC sequential heating and cooling curves for an untreated PMMA/ATH powder (a) as well as a detailed view of the second heating curve (b).

The elevated value for the PMMA/ATH in comparison to the literature value of pure PMMA of $105^{\circ} \mathrm{C}[29]$ is most probably due to the interface between the PMMA matrix and the ATH filler particles [30]. Furthermore, the glass transition temperature is dependent on the mobility of the polymer chains within the matrix. Generally, a large number or length of linear polymers' side chains leads to decreased glass transition temperatures, while stiff polymers exhibit low mobility and thus high glass transition temperatures. Accordingly, hard filler particles within the polymer matrix lead to a reduced mobility of the polymer chains and thus the glass transition temperature usually increases with an increasing content of filler material [31].

\section{Summary of PMMA/ATH powder material properties}

To summarise, the material properties of PMMA/ATH powder which was used for atmospheric pressure plasma deposition as presented hereafter are listed in Table 1. 
Table 1: Material properties of the PMMA/ATH powder used for atmospheric pressure plasma deposition.

\begin{tabular}{lr}
\hline Parameter & Value \\
\hline Ash residue in wt. \% & 53.4 \\
Constant wetting rate angle (CWRA) in degrees & $66.9 \pm 0.95$ \\
Total energy $\gamma_{\text {tot }}$ in $\mathrm{mNm}^{-1}$ & 49.2 \\
Disperse fraction $\gamma^{D}$ of $\gamma^{\text {tot }}$ in $\mathrm{mNm}^{-1}$ & 40.6 \\
Polar fraction $\gamma^{P}$ of $\gamma^{\text {tot }}$ in $\mathrm{mNm}^{-1}$ & 8.5 \\
$D_{(0,1)}$ in $\mu \mathrm{m}$ & 7.2 \\
$D_{(0,5)}$ in $\mu \mathrm{m}$ & 75.6 \\
$D_{(0,9)}$ in $\mu \mathrm{m}$ & 167.8 \\
$D_{(\max )}$ in $\mu \mathrm{m}$ & 301.6 \\
Glass transition temperature $T_{g}$ in ${ }^{\circ} \mathrm{C}$ & 120 \\
\hline
\end{tabular}

\section{Materials and Methods}

\section{Atmospheric pressure plasma layer deposition}

In order to synthesise composite PMMA/ATH, both thin and thick films on wood were realised by a thermokinetic coating technique. For this purpose, a customised plasma coating system was used. The entire setup comprises a commercially available plasma power supply and plasma generator Plasmabrush from Reinhausen Plasma $\mathrm{GmbH}$ with an optimised spraying nozzle and an aerosol generator as shown in Figure 2. Within the system, plasma process gases are ionised and form a low temperature plasma jet. Concretely, the ignition voltage is approximately $15 \mathrm{kV}$ and the effective voltage is in the range of $2-3 \mathrm{kV}$. The pulse duration amounts to $5-10 \mu \mathrm{s}$, where the pulse repetition rate is $50 \mathrm{kHz}$. Due to the transient nature of the discharge and a low current density, the plasma exhibits moderate temperatures at the exit of the nozzle that furthermore strongly decline along the direction of the gas flow. Depending on the plasma power (the maximum input power is $2 \mathrm{~kW}$ ), the kind of gas, the total gas throughput (i. e., the plasma process gas and the dispersion carrier gas), and the gas velocity, typical effluent temperatures range from 100 to $200^{\circ} \mathrm{C}$ at a working distance of $10 \mathrm{~mm}$.

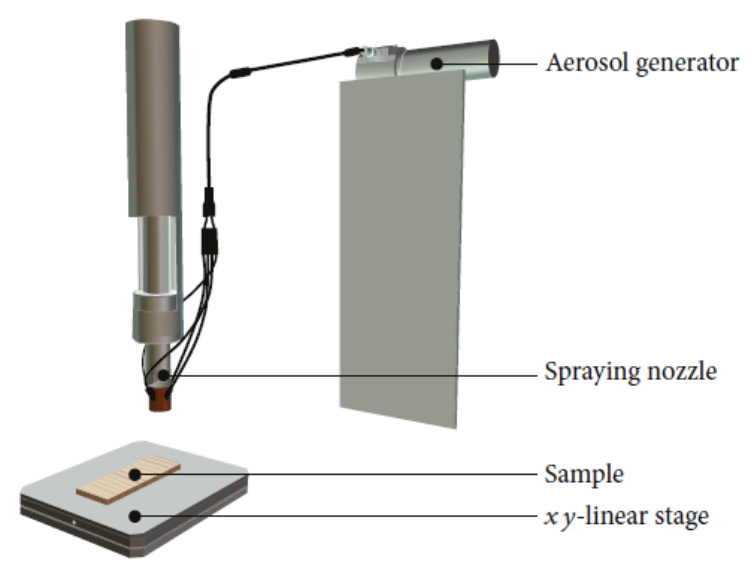

Figure 2: Setup of the used plasma coating system.

Since the starting material was dry PMMA/ATH powder, the implemented aerosol generator was used to dose, disperse, and inject particles into the plasma jet. The powder was sieved previously so that only particles with an initial diameter $<63 \mu \mathrm{m}$ were deposited. $4 \mathrm{~mm}$ thick beech wood samples (Fagus sylvatica L.) were used as substrates and placed on a $x y$-linear stage, where the lateral sample dimension was $76 \times 26 \mathrm{~mm}^{2}$. Compressed air (CA) was used as process gas and as carrier gas for the particles. The process gas flow rate and the velocity of the substrate displacement were kept constant at $30 \mathrm{~L} / \mathrm{min}$ and $20 \mathrm{~mm} / \mathrm{s}$, respectively. Different PMMA/ATH layers were deposited by varying the working distance, powder feed rate, and carrier gas pressure as listed in Table 2. 
Table 2: Parameter sets for atmospheric pressure plasma deposition of different PMMA/ATH layers.

\begin{tabular}{lccc}
\hline Sample number & $\begin{array}{c}\text { Working distance } \\
\text { in } \mathbf{~} \mathbf{m}\end{array}$ & $\begin{array}{c}\text { Powder feed rate } \\
\text { in } \mathbf{~ c m}^{\mathbf{3}} / \mathbf{h}\end{array}$ & $\begin{array}{c}\text { Carrier gas pressure } \\
\text { in bar }\end{array}$ \\
\hline $\mathrm{A}$ & 15 & 10 & 2 \\
$\mathrm{~B}$ & 13 & 18.1 & 1.5 \\
$\mathrm{C}$ & 13 & 18.1 & 2 \\
\hline
\end{tabular}

$X$-ray photoelectron spectroscopic (XPS) analysis of chemical composition

In order to determine the chemical composition of deposited PMMA/ATH coatings, X-ray photoelectron spectroscopy (XPS) was carried out at room temperature with a base pressure of $5 \times 10^{-8} \mathrm{~Pa}$ using a PHI 5000 VersaProbe II system from ULVAC-PHI. The sample surface was irradiated with an X-ray spot of $200 \mu \mathrm{m}$ in diameter, consisting of monochromatised X-ray photons (1486.8 eV with $0.26 \mathrm{eV} \mathrm{FWHM),} \mathrm{originating} \mathrm{from} \mathrm{the} \mathrm{Al} \mathrm{K}_{\alpha}$ line. Photoelectrons emitted from the sample surface were recorded via a spherical capacitor analyser. The resulting spectra were then displayed as binding energy-dependent electron intensities, where the binding energy is given with respect to the Fermi level. Broadening effects within the system add up to a total energy resolution of $<0.5 \mathrm{eV}$ (FWHM Ag3d $\mathrm{F}_{5 / 2}$ ). All spectra were recorded in constant energy mode at a pass energy of $23.5 \mathrm{eV}$ with a step size of $0.1 \mathrm{eV}$. During the measurement, charging effects were compensated via an electron flood gun $(<1 \mathrm{eV})$, running simultaneously with a low-energy $(10 \mathrm{eV}) \mathrm{Ar}^{+}$-gun.

The spectral deconvolution was carried out using the evaluation software MultiPak, where charge referencing was done by means of carbon at $284.8 \mathrm{eV}$. For quantitative analysis, sensitivity factors with correction for the analyser work function (corrected RSF) were applied after Shirley-type background correction. For the peak fitting procedure, Voigt profiles with a Lorentzian contribution of $0-22 \%$ were used in order to achieve the best agreement between experimental data and the mathematical fit. For the quantitative analysis, a total error, that is, a superposition of both the measurement and the fitting error, of $5 \%$ was assumed.

Confocal laser scanning microscopic (CLSM) analysis of topography

For the investigation of the surface topography of the deposited PMMA/ATH layers, confocal laser scanning microscopy (CLSM) was performed using a Keyence VKX210 microscope with a VKX200K controller. The wavelength of the laser used for illumination amounts to $408 \mathrm{~nm}$. The CLSM's total magnification $m_{\text {tot }}$ can be set up by varying the objective lenses $\left(m_{\mathrm{ol}}=10 \mathrm{x}, 20 \mathrm{x}, 50 \mathrm{x}\right.$, and $\left.150 \mathrm{x}\right)$ and applying the microscope's internal magnification $(10 \mathrm{x})$, an $8 \mathrm{x}$ optical zoom, and further digital magnification. The possible total magnification then amounts to $200 \mathrm{x}$ to $24,000 \mathrm{x}$. The images shown in this contribution were recorded using objectives with a magnification of $10 \times(\mathrm{NA}=0.3)$ or $150 \mathrm{x}$ $(\mathrm{NA}=0.95)$, with all of them being superpositions of widefield microscopic and laser intensity measurements.

Determination of Water Contact Angle (WCA).

Water contact angle (WCA) measurements on the PMMA/ATH coated beech samples were performed by using a commercial measuring system $\mathrm{G} 10$ from Krüss $\mathrm{GmbH}$, where each sample was measured three times. For this purpose, a droplet $(11 \mu \mathrm{L})$ of distilled water was applied to the surface and recorded for $10 \mathrm{~s}(25$ frames/sec). For every frame, the contact angle was determined by fitting a circular arc to the droplet and calculating the angle between the solid and the tangent at the point of intersection.

\section{Results and Discussion}

Chemical composition of untreated and deposited PMMA/ATH

The XPS detail spectra before background subtraction of the O1s, Al2p, and C1s peaks for both untreated powder before deposition and thick powder coating on a beech substrate (sample B) are shown in Figure 3. 


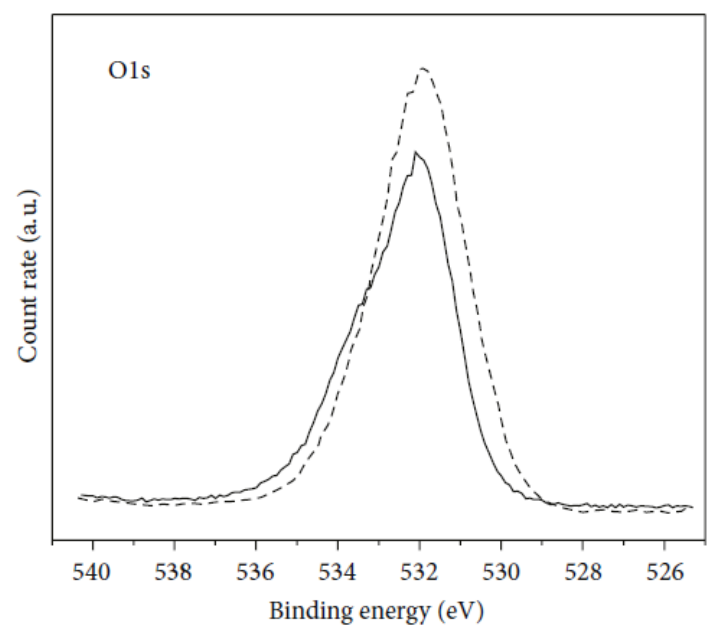

(a)

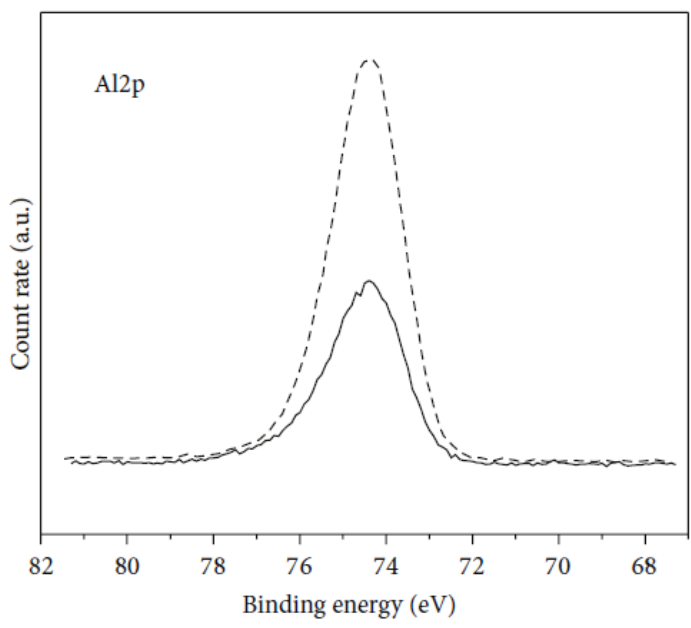

(b)

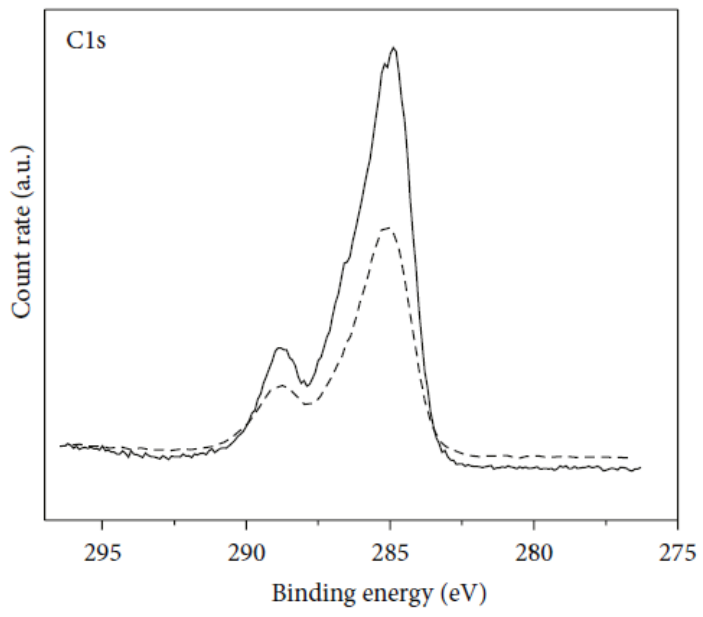

(c)

Figure 3: XPS detail spectra of untreated powder before deposition (solid line) and thick powder coating (dashed line). (a) O1s peak, (b) Al2p peak, and (c) C1s peak.

It turns out that the $\mathrm{Al} 2 \mathrm{p}$ peak intensity increases significantly after the deposition process. Consistently, the O1s peak is slightly shifted towards lower binding energies. This indicates a decrease of carbon-bound oxygen resulting from PMMA, where the O1s peaks are found at $532.01 \mathrm{eV}$ and $533.57 \mathrm{eV}$, respectively [32]. Apparently, this comes along with an increase of aluminium-bound oxygen, where the O1s peak is located at a binding energy of $531.8 \mathrm{eV}$ [33].

In order to investigate whether the increase in aluminium is due to a decomposition of PMMA, the C1s peaks represented in Figure 3(c) before and after the coating procedure were analysed in more detail as shown in Figure 4. 


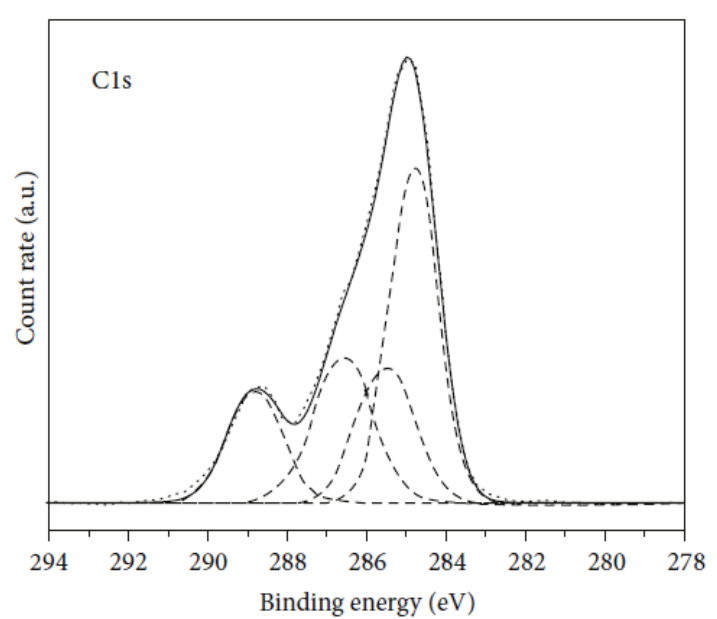

(a)

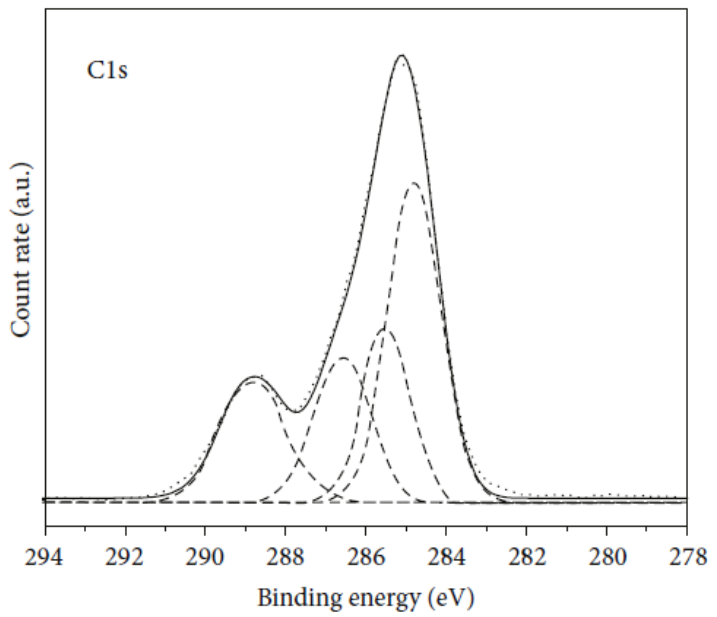

(b)

Figure 4: Measured C1s peaks of (a) untreated powder before deposition and (b) thick powder coating (dotted lines) including fits (dashed lines) for particular bonds and cumulative fit (solid lines).

For this purpose, the distances of the three minor fit peaks were constrained to $0.72 \mathrm{eV}, 1.79 \mathrm{eV}$, and $4.03 \mathrm{eV}$, respectively, to the main peak. This main peak represents the peak of lowest binding energy, hereinafter referred to as MP [32]. Moreover, the ratio of the MP's area $\left(-\mathrm{CH}_{2},-\mathrm{CH}_{3}\right)$ and that of the neighbouring peak $\left(\mathrm{C}-\mathrm{C}_{4}\right)$ were set to 0.5 [32]. Since the plasma coating procedure is expected to induce oxidation processes, the ratios of the peak areas corresponding to carbon atoms bound to oxygen are left unconstrained. The chemical compositions obtained by such spectral dissecting of the $\mathrm{C} 1 \mathrm{~s}$ peaks of the investigated surfaces, that is, untreated powder before deposition and thick powder coating, are summarised in Table 3 and Table 4.

Table 3: Binding energies (BE) of relevant peaks, particular concentration $c_{x}$, and measured relative area $A_{\text {meas }}$ of carbon bonds within the $\mathrm{C} 1 \mathrm{~s}$ peak as well as literature values for $A_{\text {lit }}$ of the untreated powder before deposition.

\begin{tabular}{lcccc}
\hline Peak & BE in $\mathbf{~ e V}$ & c in at. $\%$ & $\mathbf{A}_{\text {meas }}$ in $\%$ & $\mathbf{A}_{\text {lit }}$ in \% [32] \\
\hline $\mathrm{O} 1 \mathrm{~s}$ & 532.0 & 37.6 & & \\
$\mathrm{~A} 12 \mathrm{p}$ & 74.4 & 7.2 & & \\
$\mathrm{C} 1 \mathrm{~s}$ & & & & \\
$-\mathrm{CH}_{2}, \mathrm{CH}_{3}$ & 284.7 & 22.7 & $41.2\left(=: \mathrm{MPA}^{1}\right)$ & $42.0(=: \mathrm{MPA})$ \\
$\mathrm{C}^{-} \mathrm{C}_{4}$ & 285.5 & 11.4 & $20.6(=0.5 \cdot \mathrm{MPA})$ & $21.0(=0.5 \cdot \mathrm{MPA})$ \\
$-\mathrm{O}-\mathrm{CH}$ & 286.5 & 11.9 & $21.5(=0.5 \cdot \mathrm{MPA})$ & $21.0(=0.5 \cdot \mathrm{MPA})$ \\
$\mathrm{O}-\mathrm{C}=\mathrm{O}$ & 288.8 & 9.3 & $16.8(=0.4 \cdot \mathrm{MPA})$ & $17.0(=0.4 \cdot \mathrm{MPA})$ \\
\hline
\end{tabular}

Table 4: Binding energies (BE) of relevant peaks, particular concentration $c_{x}$, and measured relative area $A_{\text {meas }}$ of carbon bonds within the C1s peak of thick powder coating.

\begin{tabular}{lccc}
\hline Peak & BE in $\mathbf{~ e V}$ & c in at. \% & $\mathbf{A}_{\text {meas }}$ in \% \\
\hline $\mathrm{O} 1 \mathrm{~s}$ & 531.94 & 50.5 & \\
$\mathrm{~A} 12 \mathrm{p}$ & 74.30 & 15.6 & \\
$\mathrm{C} 1 \mathrm{~s}$ & & & \\
$-\mathrm{CH}_{2}, \mathrm{CH}_{3}$ & 284.78 & $13.8(=: \mathrm{MPA})$ & $40.6(=: \mathrm{MPA})$ \\
$\mathrm{C}-\mathrm{C}_{4}$ & 285.50 & $6.9(=0.5 \cdot \mathrm{MPA})$ & $20.3(=0.5 \cdot \mathrm{MPA})$ \\
$-\mathrm{O}-\mathrm{CH}$ & 286.57 & $6.4(=0.46 \cdot \mathrm{MPA})$ & $18.9(=0.46 \cdot \mathrm{MPA})$ \\
$\mathrm{O}-\mathrm{C}=\mathrm{O}$ & 288.81 & $6.9(=0.5 \cdot \mathrm{MPA})$ & $20.2(=0.5 \cdot \mathrm{MPA})$ \\
\hline
\end{tabular}

As already shown in Figure 3(b), the amount of aluminium increases in the course of the deposition process whereas the total carbon content decreases. Furthermore, an oxidation process takes place

\footnotetext{
${ }^{1}$ Main peak area
} 
yielding an increased relative amount of carbon as $\mathrm{O}-\mathrm{C}=\mathrm{O}$ compound (carboxy groups). For untreated powder, the resulting ratios of the main C1s peak's area and the other $\mathrm{C} 1 \mathrm{~s}$ peak areas are in good agreement with literature [32] as also shown in Table 3. As reported in the literature, the relative amount of methoxy groups is reduced while carboxy groups are increased but to far less extent. Hence, the arc discharge plasma used for the coating process in the present work disposes less oxidative species than the dielectric barrier discharge which was applied. In contrast to that, the resulting coatings exhibit an increased content of aluminium by a factor of about 2.2. Therefore, PMMA seems to be etched, which was also reported in [21] (cf. Section 1), and aluminium compounds are uncovered during the coating process whereas, apparently, the chemical composition of PMMA is not significantly modified. This is also confirmed by the ratios of the particular concentrations obtained from each considered peak after and before the plasma coating process $c_{c} / c_{p}$. Here, $c_{c}$ is the concentration of plasma-deposited material and $c_{p}$ is the concentration of the untreated powder corresponding to the values given in Table 3 andTable 4. Figure 5 shows a radar chart of the investigated peak ratios $c_{c} / c_{p}$. On the one hand, the data in Figure 5(a) confirm the decrease in carbon, the increase in aluminium, and the oxidation of the powder. On the other hand, Figure 5(b) reveals that, irrespective of an increase of carboxy groups, the composition of the C1s peak is not significantly changed by the plasma coating process.

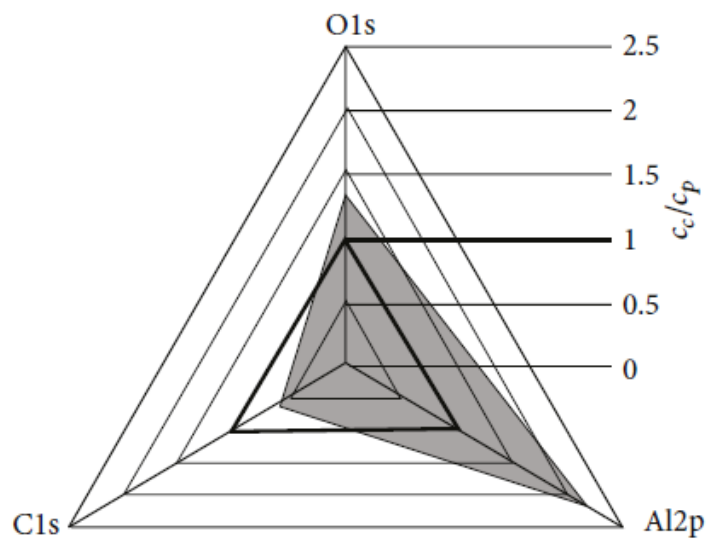

(a)

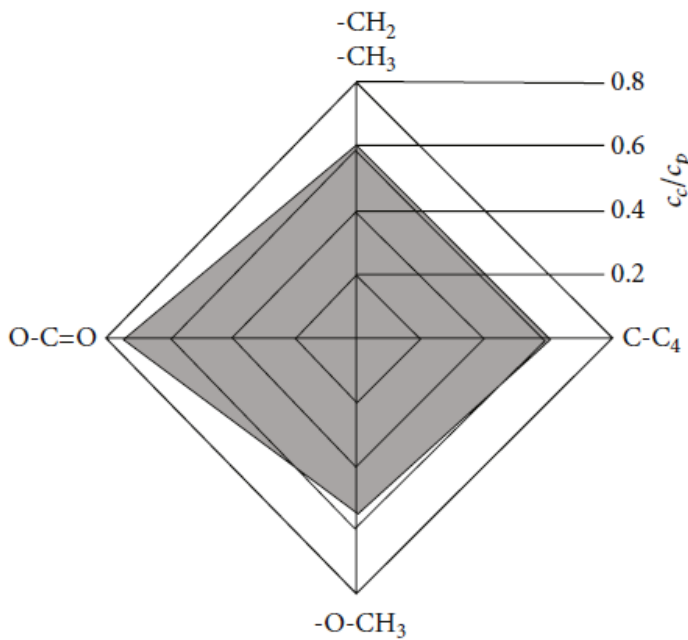

(b)

Figure 5: Radar chart of the ratios of particular concentrations obtained from each conside red peak after and before the plasma coating process $c_{\mathrm{c}} / c_{p}$.

The uncovered aluminium compounds are expected to feature a better ability to develop chemical bonds, for example, with waterborne adhesives, than the original material with a surface mainly composed of PMMA. This is also indicated by the wettability behaviour of deposited PMMA/ATH coatings as discussed hereafter.

\section{Topography}

The CLSM images of a thin plasma coating on beech wood in Figure 6(a) (top and middle image) consist of superimposed widefield microscopic and laser intensity measurements. The sample is partially covered with patches of the composite polymer material while still large areas of the substrate are exposed as shown in the top image. The bottom images display the height profile along the green line within the top image. The height of these patches amounts to about $20 \mu \mathrm{m}$ according to several of these CLSM line scans. The root means square (RMS) surface roughness $R_{\mathrm{RMS}}$ amounts to $12.5 \mu \mathrm{m}$ for the overall area shown in the top left image. On top of the white patches, the roughness evaluation yields values of $5-8 \mu \mathrm{m}$, while the uncovered substrate areas exhibit a RMS roughness of approximately $3 \mu \mathrm{m}$. A magnified detail picture of one of the white patches is additionally shown in the middle, revealing it to be roughly structured from differently sized particles. 


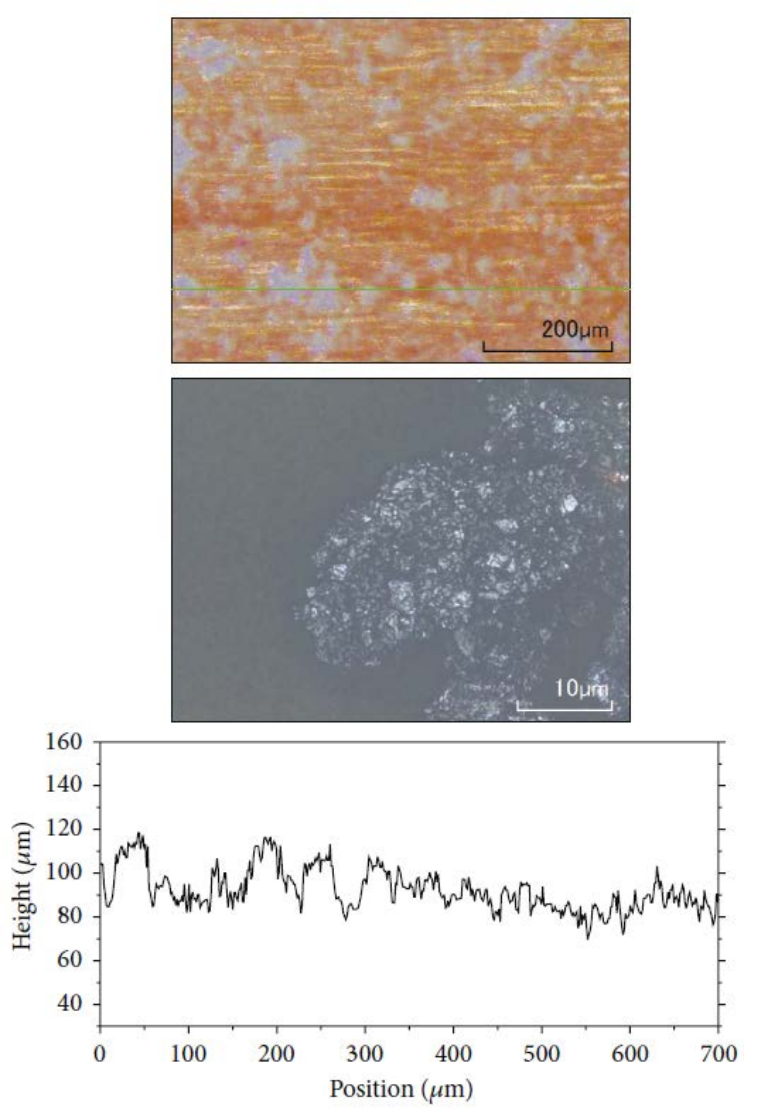

(a)
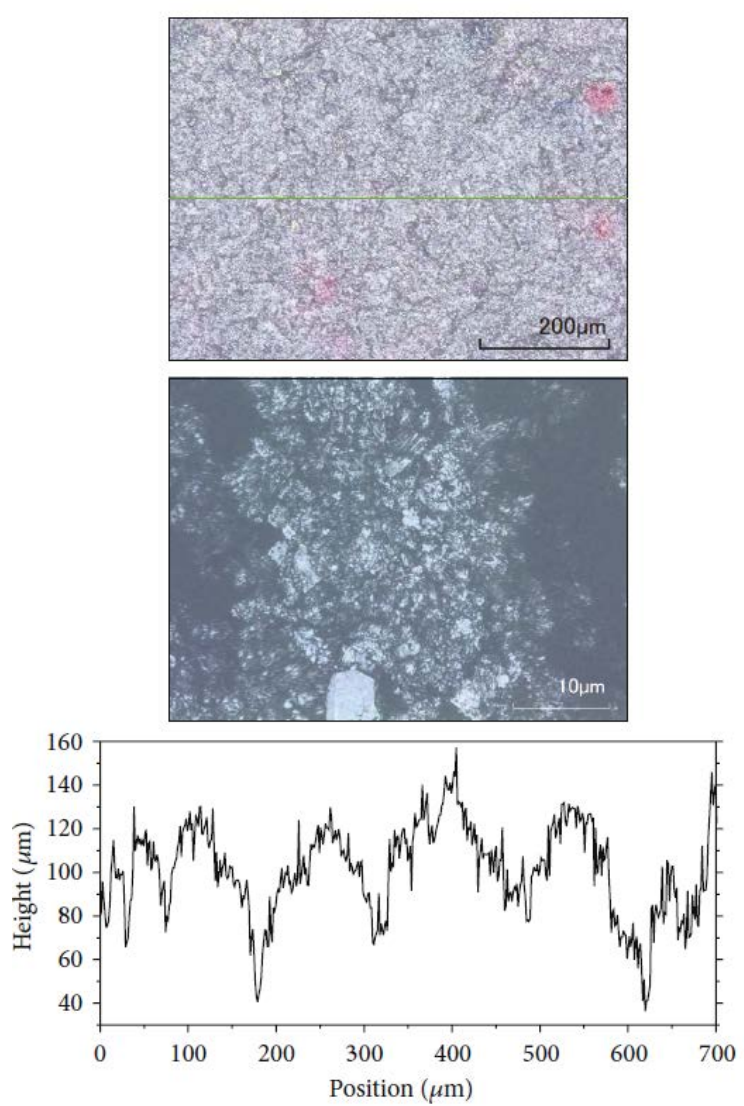

(b)

Figure 6: (a) CLSM images of a thin plasma coating on a beech wood substrate (sample A) at optical magnifications of $100 \times$ (top) and $1500 \times$ (middle) as well as a height profile along the green line within the top image. (b) CLSM images of a thick plasma coating on a beech wood substrate (sample $C$ ) at optical magnifications of $100 \mathrm{x}$ (top) and $1500 \mathrm{x}$ (middle) as well as a height profile along the green line within the top image.

Figure 6(b) shows images of a thick PMMA/ATH coating on beech wood as superimposed widefield microscopic images (top and middle) as well as a line profile (bottom image) along the green line within the top image. As visible in the top image, the surface is completely covered with the deposited composite polymer material. The red spots are due to residuals from differently coloured powder of the same base material. The magnified detail picture (middle) reveals a rough structure with differently sized, separate particles just as for the partially coated sample. The bottom image depicts the height profile along the green line within the top image. This line scan shows elevations with diameters of around $100 \mu \mathrm{m}$ and heights of approximately $30-100 \mu \mathrm{m}$, which are not correlated with the structure of the underlying beech wood substrate. The RMS roughness amounts to $R_{\mathrm{RMS}}=25.2 \mu \mathrm{m}$, thus encouraging the visual impression of a heavily ragged coating.

The presence of separate particles within both coatings is rather surprising, since the glass transition temperature of the PMMA/ATH powder was determined to be about $120^{\circ} \mathrm{C}$. The gas temperatures within the active region of the plasma device usually exceed $1000 \mathrm{C}$, while the remote plasma mostly exceeds the given glass transition temperature, even at a distance of $10 \mathrm{~mm}$ from the plasma jet nozzle (cf. Section 3). Considering the retention time of the particles within the hot gas stream, the particle temperatures should well exceed the glass transition temperature, too, and thus the deformation of the particles as well as the mobility of the polymer chains should be large enough to assure an interdiffusion of the polymer chains and thereby the film formation process. However, the particles obviously did largely neither deform in shape nor form a closed film. Even though this should happen even for slightly cross-linked polymers, further experiments showed that, at temperatures as high as $200^{\circ} \mathrm{C}$, no interdiffusion takes place for the used PMMA/ATH composite material. This might most likely be due to the chemical bonds between the PMMA and the ATH via the used functional silanebased surfactant. These findings also fit very well to the high temperature resistance of PMMA and the nearly temperature-independent modulus of elasticity up to thermal decomposition of the 
polymer [34]. Furthermore, there might be an influence due to the effects of the plasma treatment beyond the heat transfer, for example, the etching of the PMMA polymer. A significant formation of cross-links can be excluded from the XPS results since, here, no notable changes in the bonding structure of the carbon compounds could be determined (cf. Figure 4 and Figure 5). Even though there is still a significant amount of PMMA left after the plasma treatment, the assumed polymer etching leads to a considerably increased fraction of the filler particles as compared to the polymer matrix (cf. Section 4, chemical analysis). Thus, the glass transition temperature should further increase [31]. The main etching mechanism is the generation of short, volatile oligomers via chain scission [35], which might possibly also lead to the formation of residual short-chained oligomers at the particle surfaces. The interdiffusion and film formation processes, however, depend strongly on the interface between the PMMA/ATH particles and might therefore be significantly hindered by an adlayer of shortchained oligomers.

\section{Wettability}

The evolution of the water contact angles versus time is represented in Figure 7. For each time interval, the average of the three measurements was plotted. As a cut-off, the shortest time of complete soaking was used. This point in time represents the limit of measurability which is found at a water contact angle of $<5^{\circ}$. Each droplet was completely soaked by the material within the measuring period of $10 \mathrm{~s}$, illustrating a high wettability of the plasma-deposited PMMA/ATH coatings. Generally, the contact angles for the thin coating (Figure 7(a), cf. also Figure 6(a)) are smaller than for the thick coatings (Figure 7(b) andFigure 7(c), cf. also Figure 6(b)) for all time intervals. This is supposed to be caused by a surface activation of the substrate itself, that is, beech wood, as already reported, for example, by Wolkenhauer and coworkers [36].

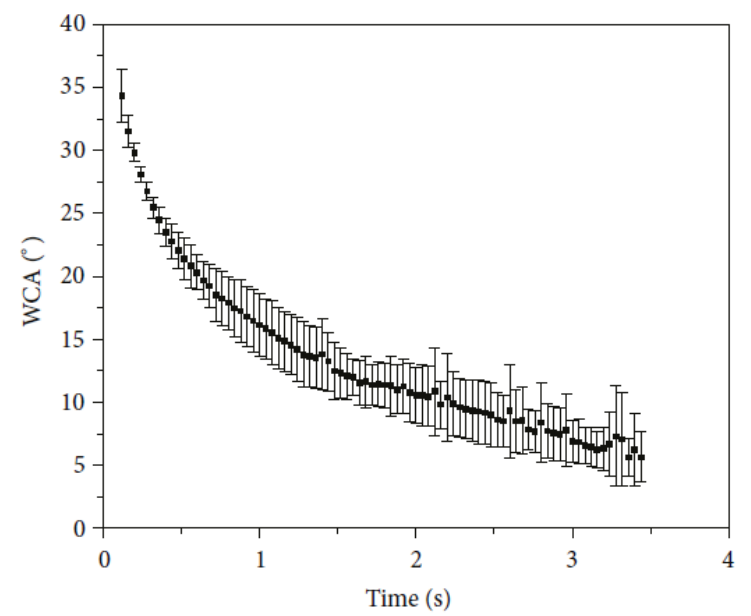

(a)

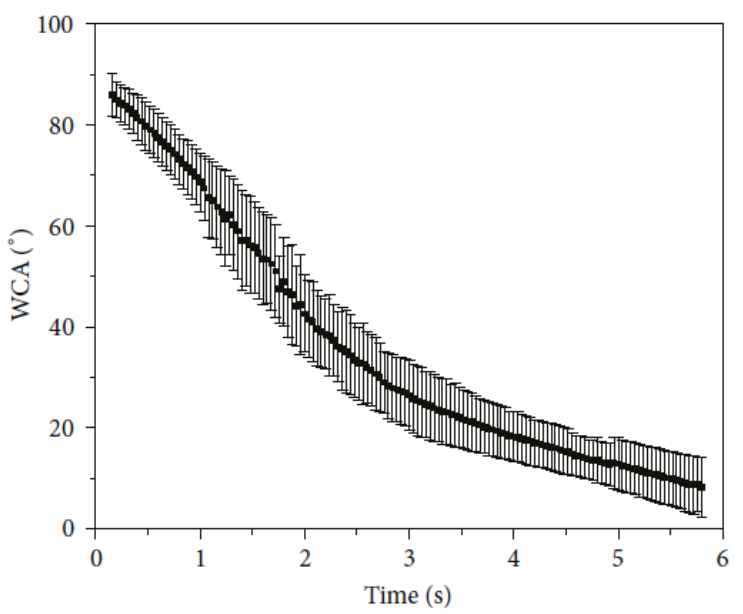

(b) 


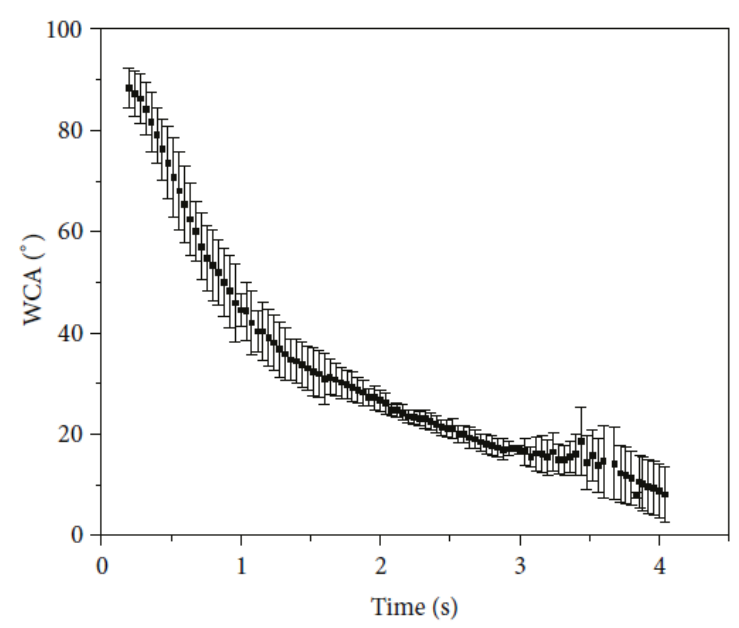

(c)

Figure 7: Evolution of the water contact angles for (a) thin coating (sample A) and thick coatings: (b) sample B, (c) sample C.

In the literature, contact angles for untreated plates were found to be $>60^{\circ}$ and no soaking process was observed. The raw material is thus rather hydrophobic which is generally also valid for pure PMMA. This behaviour is confirmed by the measurements of the constant wetting rate angle on pressed powder pellets before plasma deposition (cf. Section 2). Even though a quantitative comparison of the wettability of the PMMA/ATH plates, untreated powder, and the deposited coatings is not possible (which is due to the completely different forms, either in powder form or in solid matter), the highly hydrophilic characteristics of all plasma coatings obviously have to be correlated with a significantly increased polar part of the surface energies. Moreover, diiodomethane droplets $(8 \mu \mathrm{L})$ were applied to study the wettability of the coatings with a nonpolar liquid, but all droplets were soaked almost immediately without yielding measurable contact angles. Hence, the resulting coatings also exhibit highly lipophilic characteristics similar to the starting material.

The improved wettability is in good agreement with the results obtained by XPS. As a result of an uncovering of aluminium compounds, the wettability is enhanced by providing new bonding sites. Furthermore, the rough structure of the surface confirmed by CLSM measurements also supports this effect. In contrast to the literature, the PMMA polymer matrix of the deposited layers is not oxidised by the attachment of polar groups at all, which could contribute to the wettability with water.

\section{Summary and Outlook}

In this work, it was shown that PMMA/ATH raw material and plasma-deposited PMMA/ATH feature notably different material properties. As a main aspect, the XPS investigations suggest that ATH was uncovered by a plasma-induced etching of the PMMA matrix in the course of the plasma deposition process as visualised in Figure 8.

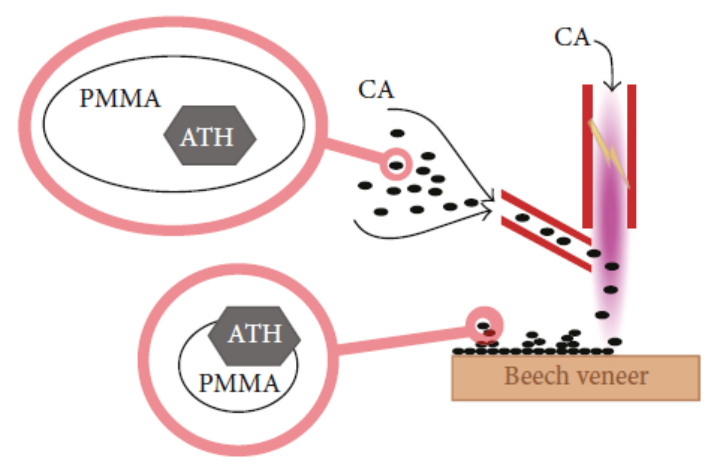

Figure 8: Visualisation of the plasma-induced modification of PMMA/ATH by atmospheric pressure plasma powder deposition. 
However, based on the given results, it cannot be stated whether the ATH particles are chemically influenced by the plasma process, too, or remain as aluminium hydroxide. A more detailed investigation using Auger electron spectroscopy is planned to be carried out in ongoing work. In any case, the supplementary water contact angle measurements support an uncovering of aluminium-based bonds. In contrast to the raw material which shows a hydrophobic behaviour, the deposited coatings are rather amphiphilic. As a result of this property, the surface energy could not be determined since the analysis of contact angles of dispersed liquids was not possible. It was shown that such high wettability is not caused by the formation of additional polar groups, that is, an activation of PMMA, but most likely due to the above-mentioned uncovering of ATH. The observed decrease in carbon, giving rise to such uncovering, is most probably due to a chain scission mechanism from the plasma as described, for example, by Kuzuya and coworkers [35]. Subsequently, the resulting short-chained oligomers would desorb from the PMMA/ATH particles' surfaces. An adjacent oxidation of these hydrocarbon molecules by the plasma towards the formation of $\mathrm{CO}_{2}$ and water is likely but has not been investigated yet.

As shown by CLSM, the plasma deposition does not result in film formation. Thus, completed coatings cannot be realised by simply applying this approach. However, the uncovering of ATH provides further docking points that might improve the adhesion of glues or lacquers. In future projects, the realised coatings could thus be finished by applying either solvent- or water-based lacquers while a complete impregnation of the PMMA/ATH primary coating should be ensured due to its high wettability for polar and nonpolar liquids. In such a multicomposite system, the ATH particles could significantly improve the mechanical properties of the coating as commonly known for inorganic filler particles within polymers [8-10]. Furthermore, the ATH particles should significantly promote the adhesion between both a polar top coat matrix and the PMMA particles, due to form closure as well as due to the ATH particles being chemically bonded to the finish coating and via silane-based surfactants to the PMMA. Another finishing procedure could include a polymerization of further MMA monomers around the deposited coatings in order to maintain the excellent chemical stability and resistance against UV radiation of the raw material. This might even be possible by plasma polymerization via the addition of the MMA monomer (cf. [37]) to the process gas during the PMMA/ATH powder-based primary film deposition, which will be evaluated in a consecutive study. The development of such layer systems involving atmospheric pressure plasma powder deposition of PMMA/ATH could contribute to sustainable and resource-efficient coating processes since the raw material is recycled from waste, that is, abrasive dust which originates from classical manufacturing of PMMA/ATH work pieces as already mentioned in the Introduction.

\section{Acknowledgements}

This work was supported by the German Federal Ministry of Education and Research in the frame of the research project "PlaNaWood," funding reference no. 03X5519B. The authors further thank Frank Endres from the Institute of Electrochemistry at Clausthal University of Technology for the provision of the CLSM, Ulrike Koecher from the Institute of Technical Chemistry at Clausthal University of Technology for the measurement of DSC curves, and Roman Rerich from the University of Applied Sciences and Arts for their help during the experimentation and evaluation as well as Andrej Beličič from Kolpa d. d. for providing the used powder material.

\section{References}

[1] M. Wagner and J. Sellers, "Kinetics of filler-polymer interaction between fine particle silica and SBR or butyl rubber," Industrial \& Engineering Chemistry, vol. 51, no. 8, pp. 961-966, 1959.

[2] H. Zhang, M. Lu, Q. Lu et al., "Design and preparation of cross-linked $\alpha$-methylstyrene acrylonitrile copolymer nanoparticles and their interfacial investigation with rubber," Journal of Applied Polymer Science, vol. 132, no. 18, 2015.

[3] "Plastics - Decorative solid surfacing materials - Part 2: Determination of properties - Sheet goods," 2007, ISO 19712-2.

[4] R. B. Duggins, C. Ford, H. C. Miller et al., US 3827933: Filled polymethyl methacrylate article and a process for its manufacture, 1974. 
[5] R. B. Duggins and C. Ford, US 3847865: Use of alumina trihydrate in a polymethyl methacrylate article, 1974.

[6] C. R. Peters and J. E. Young, US 6554944B1: Process for making bowls comprising acrylic plastics filled with alumina trihydrate, 2003.

[7] E. Gunel and C. Basaran, "Influence of filler content and interphase properties on large deformation micromechanics of particle filled acrylics," Mechanics of Materials, vol. 57, pp. 134-146, 2013.

[8] O. A. Stapountzi, M. N. Charalambides, and J. G. Williams, "Micromechanical models for stiffness prediction of alumina trihydrate (ATH) reinforced poly (methyl methacrylate) (PMMA): Effect of filler volume fraction and temperature," Composites Science and Technology, vol. 69, 11-12, pp. 2015-2023, 2009.

[9] C. Basaran, S. Nie, and C. S. Hutchins, "Time dependent behavior of a particle filled composite PMMA/ATH at elevated temperatures," Journal of Composite Materials, vol. 42, no. 19, pp. 2003-2025, 2008.

[10] C. Basaran, S. Nie, C. S. Hutchins et al., "Influence of interfacial bond strength on fatigue life and thermo-mechanical behavior of a particulate composite: An experimental study," International Journal of Damage Mechanics, vol. 17, no. 2, pp. 123-147, 2007.

[11] I. Sobolev and Woycheshin E. A., "Alumina trihydrate," in Handbook of Fillers for Plastics, Katz. H. S., Ed., Van Nostrand Reinhold, New York, 1987.

[12] H. S. Katz and J. V. Milewski, Handbook of Fillers for Plastics, Van Nostrand Reinhold, New York, 1987.

[13] A. P. Mouritz and A. G. Gibson, "Flame retardant composites," in Fire Properties of Polymer Composite Materials, A. P. Mouritz and A. G. Gibson, Eds., pp. 237-286, Springer, Dordrecht, 2006.

[14] O. Obakponovwe and J. G. Williams, "Temperature effects on the fatigue of highly filled PMMA," Journal of Materials Science, vol. 41, no. 2, pp. 437-443, 2006.

[15] I. M. Joni, T. Nishiwaki, K. Okuyama et al., "Enhancement of the thermal stability and mechanical properties of a PMMA/aluminum trihydroxide composite synthesized via bead milling," Powder Technology, vol. 204, no. 1, pp. 145-153, 2010.

[16] E. P. Plueddemann, Silane Coupling Agents, Plenum Press, New York, 1991.

[17] V. Jivkov, R. Simeonova, A. Marinova et al., "Study on the gluing abilities of solid surface composites with different wood based materials and foam PVC," in Proceedings of the 24th International Scientific Conference, pp. 49-55.

[18] E. Šušteršič, M. Tušar, and A. Zupančič Valant, "Rheological and mechanical characterization of waste PMMA/ATH modified bitumen," Construction and Building Materials, vol. 38, pp. 119_ $125,2013$.

[19] W. Kaminsky, M. Predel, and A. Sadiki, "Feedstock recycling of polymers by pyrolysis in a fluidised bed," Polymer Degradation and Stability, vol. 85, no. 3, pp. 1045-1050, 2004.

[20] M. C. Sunny and C. P. Sharma, "Surface modification of corneal contact lens with phosphoryl choline by glow discharge," Biomaterials, Artificial Cells and Immobilization Biotechnology, vol. 19, no. 3, pp. 599-612, 2009.

[21] K. Saito and A. Yoshikawa, " $\mathrm{O}_{2}$ plasma etch rate reduction on synchrotron radiation exposed PMMA film," Japanese Journal of Applied Physics, vol. 26, no. 9, 1428-1430, 1987.

[22] M. Collaud Coen, R. Lehmann, P. Groening et al., "Modification of the micro- and nanotopography of several polymers by plasma treatments," Applied Surface Science, vol. 207, 14, pp. 276-286, 2003.

[23] A. Fridman, S. Nester, L. A. Kennedy et al., "Gliding arc gas discharge," Progress in Energy and Combustion Science, vol. 25, no. 2, pp. 211-231, 1999.

[24] U. Kogelschatz, "Dielectric-barrier discharges: their history, discharge physics, and industrial applications," Plasma Chemistry and Plasma Processing, vol. 23, no. 1, pp. 1-46, 2003.

[25] R. Wang, C. Zhang, X. Liu et al., "Microsecond pulse driven $\mathrm{Ar} / \mathrm{CF}_{4}$ plasma jet for polymethylmethacrylate surface modification at atmospheric pressure," Applied Surface Science, vol. 328, pp. 509-515, 2015.

[26] G. Oberbossel, A. T. Güntner, L. Kündig et al., "Polymer powder treatment in atmospheric pressure plasma circulating fluidized bed reactor," Plasma Processes and Polymers, vol. 12, no. 3, pp. 285-292, 2015. 
[27] D. K. Owens and R. C. Wendt, "Estimation of the surface free energy of polymers," Journal of Applied Polymer Science, vol. 13, no. 8, pp. 1741-1747, 1969.

[28] R. M. Nussbaum, "Natural surface inactivation of scots pine and norway spruce evaluated by contact angle measurements," European Journal of Wood and Wood Products, vol. 57, no. 6, pp. 419-424, 1999.

[29] J. Brandrup, ed., Polymer Handbook., Wiley, Hoboken, NJ, 1999.

[30] J. Berriot, H. Montes, F. Lequeux et al., "Evidence for the shift of the glass transition near the particles in silica-filled elastomers," Macromolecules, vol. 35, no. 26, pp. 9756-9762, 2002.

[31] S. Cerveny, S. N. Goyanes, A. J. Marzocca et al., "Dynamic properties in aluminum filled PMMA," Polymer, vol. 40, no. 6, pp. 1495-1500, 1999.

[32] G. Beamson and D. Briggs, High Resolution XPS of Organic Polymers, Wiley, 1992.

[33] J. T. Kloprogge, L. V. Duong, B. J. Wood et al., "XPS study of the major minerals in bauxite: gibbsite, bayerite and (pseudo-)boehmite," Journal of Colloid and Interface Science, vol. 296, no. 2, pp. 572-576, 2006.

[34] M. J. Forrest, Analysis of Plastics, Smithers Rapra, Shrewsbury, 2002.

[35] M. Kuzuya, A. Noguchi, M. Ishikawa et al., "Electron spin resonance study of free-radical formation and its decay of plasma-irradiated poly (methacrylic acid) and its esters," The Journal of Physical Chemistry, vol. 95, no. 6, pp. 2398-2403, 1991.

[36] A. Wolkenhauer, G. Avramidis, E. Hauswald et al., "Sanding vs. plasma treatment of aged wood: A comparison with respect to surface energy," International Journal of Adhesion and Adhesives, vol. 29 , no. 1, pp. 18-22, 2009.

[37] T. B. Casserly and K. K. Gleason, "Effect of substrate temperature on the plasma polymerization of poly(methyl methacrylate)," Chemical Vapor Deposition, vol. 12, no. 1, pp. 59-66, 2006. 


\title{
Paper IV
}

\section{Enhancing the Abrasion Resistance of PMMA/ATH Layers realised by means of Atmospheric Pressure Plasma Powder Deposition on Wood}

\author{
Lena Wallenhorst ${ }^{1}$, Roman Rerich ${ }^{1}$, Matej Vovk ${ }^{2}$, Sebastian Dahle ${ }^{3}$, Holger Militz ${ }^{4}$, \\ Gisela Ohms ${ }^{1}$, Wolfgang Viöl ${ }^{1,5}$
}

${ }^{1}$ University of Applied Sciences and Arts, Laboratory of Laser and Plasma Technologies, Von-Ossietzky-Straße 99, 37085 Göttingen, Germany

${ }^{2}$ University of Ljubljana, Biotechnical Faculty, Jamnikarjeva 101, 1000 Ljubljana, Slovenia

${ }^{3}$ Clausthal University of Technology, Institute of Energy Research and Physical Technologies, Leibnizstraße 4, 38678 Clausthal-Zellerfeld, Germany

4 Georg-August-University Göttingen, Wood Biology and Wood Products, Burckhardt Institute, Büsgenweg 4, 37077 Göttingen, Germany

${ }^{5}$ Fraunhofer Institute for Surface Engineering and Thin Films, Appl. Center for Plasma and Photonics, Von-Ossietzky-Straße 100, 37085 Göttingen, Germany

\begin{abstract}
In this work, we present the deposition and stabilisation of PMMA/ATH powder on wood and glass substrates in a cold atmospheric pressure plasma-spray process. For this purpose, the raw powder material was injected to the effluent plasma of a jet discharge to coat the substrate that is situated in a comparatively cold region of the afterglow. Since the abrasion resistance of pure PMMA/ATH powder coatings deposited with the use of compressed air as process and carrier gas turns out to be very low, two variations to stabilise those coatings were tested. The replacement of compressed air as process and carrier gas by forming gas did not lead to an increased abrasion resistance, but the addition of phenol-formaldehyde (PF) powder successfully stabilised the coatings.
\end{abstract}

Keywords: Plasma Coatings, PMMA/ATH, Abrasion, Recycling

\section{Introduction}

Poly(methyl methacrylate) (PMMA) reinforced with aluminium trihydrate $\left(\mathrm{Al}(\mathrm{OH})_{3}, \mathrm{ATH}\right)$ is classified as solid surface material[1] with characteristics meeting the requirements of the performance standard ANSI/ICPA SS-1-2001. The PMMA/ATH composite, as it is abbreviated, was developed and described by Duggins and co-workers [2,3]. In general, the composites contain about 50 to $70 \mathrm{wt} . \%$ of ATH filler and about 30 to $50 \mathrm{wt} . \%$ of PMMA resin. Because of this composition, machining of PMMA/ATH composite is very similar to machining of wood, but PMMA/ATH can additionally be thermoformed similar to thermoplastics. Unlike wood, though, it exhibits a high moisture resistance along with both a good chemical and UV resistance. These properties make PMMA/ATH composites widely useable as working surfaces and claddings for indoor and outdoor applications.

However, about $20 \%$ of the material produced is represented in post-industrial waste, leading to a production of about 1000 tons of PMMA/ATH waste every year only in Slovenia. Cut-offs and powder waste of PMMA/ATH material is generated during the post-polymerization process by trimming and sanding the PMMA/ATH sheets. Different methods of recycling that particular waste 
have already been considered [4-10], but none of them have provided a simple, sustainable and costeffective solution yet. In previous studies [8], PMMA/ATH powder was deposited in a plasma-spray process by the use of air as process and carrier gas. A functionalisation of the used PMMA/ATH material could be detected; however, it did not yield abrasion resistant coatings. Therefore, the use as protective coatings on wood was not possible. In the present study, the replacement of air by forming gas was investigated which could yield more reactive molecule fragments and consequently improve the adhesion.

Our preliminary research suggested that PMMA/ATH boards and waste material are compatible with phenol formaldehyde (PF) resin, yielding the opportunity to use this resin in a production of some kind of recycled composite. PF resins are most often used in wood composite adhesives [11]. It has a unique ability to react with formaldehyde in acidic or basic conditions [12]. The former gives resole resins, while the reaction in basic conditions yields novolak resins [13]. Powdered novolak PF can easily be combined with the PMMA/ATH powder for the application by atmospheric pressure plasma powder deposition. The use of powdered phenol novolak resin with a medium hexamethylenetetramine (HMTA) content and medium flow turned out to be appropriate for the intended application. This kind of resin was intentionally formulated as a binding agent for the manufacturing of hot or highly compressed cold pressed grinding wheels, where high thermal resistance and toughness is required. The performance of powdered phenolic resin is roughly determined by its molecular weight, which influences flow, hexamine content and particle size [12]. The resin used in this study melts at about $90^{\circ} \mathrm{C}$ and reacts at $130^{\circ} \mathrm{C}$. When cured, it has high dry and wet strength and is very resistant to water and damp atmospheres [11].

\section{Objectives}

The main objective of this study was the stabilisation of plasma coatings based on PMMA/ATH powder as protective coating on wood from a recycled waste powder.

\section{Methods, Material and Equipment}

Atmospheric pressure plasma layer deposition

To deposit composite PMMA/ATH layers, a setup as described in $[8,14,15]$ was used which is based on the Plasmabrush from Reinhausen Plasma GmbH with a modified spraying nozzle. Briefly, the powder was added to the afterglow of a jet discharge driven by pulsed high voltage (ignition voltage: approx. $15 \mathrm{kV}$, effective voltage: $2-3 \mathrm{kV}$, max. input power: $2 \mathrm{~kW}$, pulse duration: $5-10 \mu \mathrm{s}$, pulse repetition rate: $50 \mathrm{kHz}$ ). To generate the particle aerosol, the dry powder was homogeneously compressed in a cylindrical container and moved towards a rotating brush at a constant velocity defining the feed rate. Above the brush, the carrier gas stream took up the particles. The PMMA/ATH powder was sieved prior to utilisation, yielding initial diameters below $70 \mu \mathrm{m}$. The samples were placed on an $x-y$ linear stage that moved them below the spraying nozzle to ensure an overall homogeneous coating.

Further, in an attempt to improve the abrasion resistance, two different modifications of this basic deposition process were investigated:

A. Use of pure PMMA/ATH powder with compressed air as process and carrier gas,

B. Use of forming gas instead of compressed air as process and carrier gas,

C. Addition of $10 \%$ phenol-formaldehyde powder (PF, Borofen BL-35 from Kolpa d.d) to the PMMA/ATH powder.

The coatings were deposited on beech wood (Fagus sylvatica L., $4 \times 76 \times 26 \mathrm{~mm}^{3}$ ) as well as on sodalime glass (microscope slides) as a model substrate for surfaces that cannot be coated easily. The parameters applied during the deposition process are listed in Table 1; for each coating system, two replicates were prepared. Since every substance that was deposited in this plasma process requires different parameters to ensure homogenous coatings and to keep the substrates' temperatures low enough to avoid thermal damage at the same time, the deposition parameters cannot be kept the same for all coating systems. 
Table 1: Summary of coating parameters (subscript w stands for wood, g for glass).

\begin{tabular}{|c|c|c|c|c|c|c|}
\hline $\begin{array}{l}\text { Sample } \\
\text { No. }\end{array}$ & $\begin{array}{c}\text { Deposited } \\
\text { material }\end{array}$ & $\begin{array}{c}\text { Process/carrier } \\
\text { gas }\end{array}$ & $\begin{array}{c}\text { Carrier } \\
\text { gas } \\
\text { pressure } \\
\text { in bar } \\
\end{array}$ & $\begin{array}{c}\text { Working } \\
\text { distance } \\
\text { in } \mathbf{~ m m}\end{array}$ & $\begin{array}{c}\text { Powder } \\
\text { feed rate } \\
\text { in } \mathrm{cm}^{3 / h}\end{array}$ & $\begin{array}{c}\text { Substrate } \\
\text { displacemen } \\
\text { in } \mathrm{mm} / \mathrm{s}\end{array}$ \\
\hline$A_{w / g}$ & PMMA/ATH & $\begin{array}{l}\text { Compressed air } \\
(401 / \mathrm{min})\end{array}$ & 1.5 & 13 & 72 & 20 \\
\hline $\mathrm{B}_{\mathrm{w} / \mathrm{g}}$ & PMMA/ATH & $\begin{array}{l}\text { Forming gas } \\
(351 / \mathrm{min})\end{array}$ & 1.0 & 25 & 121 & 100 \\
\hline $\mathrm{C}_{\mathrm{w} / \mathrm{g}}$ & $\begin{array}{c}\text { PMMA/ATH } \\
(90 \%) \\
\text { PF }(10 \%)\end{array}$ & $\begin{array}{l}\text { Compressed air } \\
\text { (40 1/min) }\end{array}$ & 1.6 & 16 & 121 & 20 \\
\hline
\end{tabular}

\section{Abrasion tests}

To study the abrasion resistance, a setup according to DIN ISO 9211-4 was used. Here, cotton tissues were fixed on a test probe and moved on the sample with a pressure of $(5 \pm 1) \mathrm{N}$. One cycle is defined as one movement backwards followed by one movement forwards. The samples were evaluated visually after taking a picture with an EOS 600D digital camera (Canon Inc.).

\section{Results and Discussion}

Figure 1 shows pictures of the three different coating systems on wood after one abrasion cycle. Except for some particles that remained fixed in the wood's rough surface, the two coatings $A_{w}$ and $\mathrm{B}_{\mathrm{w}}$ were already removed, hence, exchanging compressed air as process/carrier gas by forming gas does not improve the abrasion resistance. In contrast, only slight changes could be observed for the coating consisting of PMMA/ATH and PF. Similarly, coating systems $\mathrm{A}_{\mathrm{g}}$ and $\mathrm{B}_{\mathrm{g}}$ on glass substrates (not shown) were completely removed after 1 cycle whereas system $C_{g}$ showed only little removal of substance.

For the coating system containing PMMA/ATH and PF, the abrasion tests were extended. Figure 2 shows coated glass and coated wood surface after subjection to 1000 abrasion cycles, still with a significant amount of coating on the ablation site. For wood, the coating could be removed after approximately 2000 cycles, whereas no complete removal of the coating on glass was possible (up to 4400 cycles were tested on each sample). Hence, both, the adhesion between the particles that constitute the coating as well as the adhesion between the coating and the surfaces, were improved.

The poorer abrasion resistance in the case of wood substrates might be explained by the lower mechanical stability of wood which could lead to wood failure instead of a failure of the adhesive bond. Laborie and Frazier [16] proposed secondary interactions between the wood constituents and the hydroxyl groups of the resin as mechanism explaining the adhesion between wood and PF. 


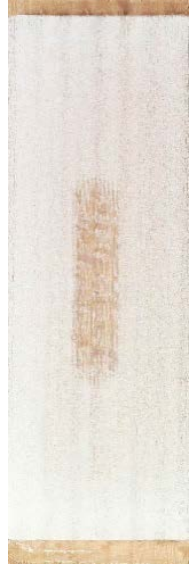

(a)

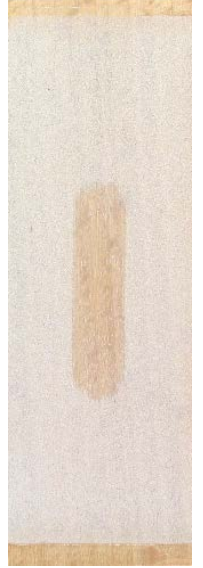

(b)

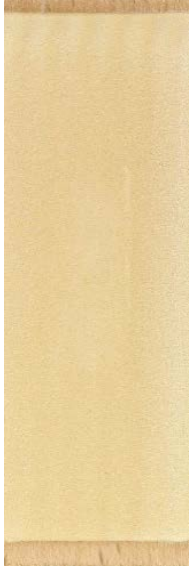

(c)

Figure 1: Coating after 1 cycle. (a): sample $A_{w}$ (PMMA/ATH with compressed air); (b): sample B $_{w}$ (PMMA/ATH with forming gas); (c) sample $\mathrm{C}_{\mathrm{w}}$ (PMMA/ATH + PF with compressed air).

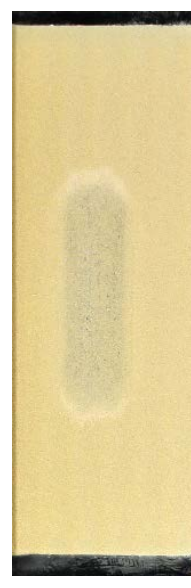

(a)

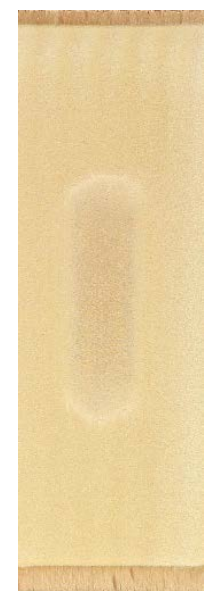

(b)

Figure 2: PMMA/ATH + PF coating after 1000 cycles on glass (Fig. 2(a)) and wood (Fig. 2(b)).

\section{Conclusions}

In this article, a way to deposit abrasion-resistant coatings on wood and glass via the addition of phenol-formaldehyde powder to the PMMA/ATH raw powder was presented. The PMMA/ATH + PF coatings withstand approx. 2000 abrasion cycles on wood, whereas it was not even possible to completely remove the coatings from glass substrates by the method applied. To understand the mechanisms behind this significantly improved abrasion resistance, detailed studies on the chemical and structural properties are subject to further ongoing investigations.

\section{Acknowledgements}

We gratefully acknowledge financial support from the Lower Saxony Ministry of Science and Culture (MWK). The authors further thank Kolpa d. d. for providing the coating materials as well as Christoph Gerhard for proofreading the manuscript. 


\section{References}

[1] "Plastics - Decorative solid surfacing materials - Part 2: Determination of properties - Sheet goods," 2007, ISO 19712-2.

[2] R. B. Duggins and C. Ford, US 3847865: Use of alumina trihydrate in a polymethyl methacrylate article, 1974.

[3] R. B. Duggins, C. Ford, H. C. Miller et al., US 3827933: Filled polymethyl methacrylate article and a process for its manufacture, 1974.

[4] W. Kaminsky and J. Franck, "Monomer recovery by pyrolysis of poly(methyl methacrylate) (PMMA)," Journal of Analytical and Applied Pyrolysis, vol. 19, pp. 311-318, 1991.

[5] W. Kaminsky, M. Predel, and A. Sadiki, "Feedstock recycling of polymers by pyrolysis in a fluidised bed," Polymer Degradation and Stability, vol. 85, no. 3, pp. 1045-1050, 2004.

[6] J. Hochberg and R. B. Young, US 4759774 A: Process for cleaning substrates, 1988.

[7] E. Šušteršič, M. Tušar, and A. Zupančič Valant, "Rheological and mechanical characterization of waste PMMA/ATH modified bitumen," Construction and Building Materials, vol. 38, pp. 119$125,2013$.

[8] L. M. Wallenhorst, S. Dahle, M. Vovk et al., "Characterisation of PMMA/ATH layers realised by means of atmospheric pressure plasma powder deposition," Advances in Condensed Matter Physics, vol. 2015, no. 3, pp. 1-12, 2015.

[9] M. Tušar, E. Šušteršič, and A. Zupančič-Valant, "Alternative PMB produced from recycling waste PMMA/ATH," Transport Problems, vol. 9, no. 2, pp. 5-9, 2014.

[10] M. Tušar, A. Beličič, M. Prešeren et al., EP 20130730823: Modified bitumen and its use for preparing asphalt mixtures and bituminous products, 2015.

[11] United States Department of Agriculture, Wood Handbook: Wood as an Engineering Material, Forest Product Laboratory, USDA, Madison, 2010.

[12] L. Pilato, Phenolic Resins: A Century of Progress, Springer, Heidelberg, London, New York, 2010.

[13] K. Mittal and A. Pizzi, Handbook of Adhesive Technology, Dekker, New York, 2003.

[14] P. Gascón-Garrido, N. Mainusch, H. Militz et al., "Copper and aluminium deposition by coldplasma spray on wood surfaces: effects on natural weathering behaviour," European Journal of Wood and Wood Products, vol. 75, no. 3, pp. 315-324, 2016.

[15] P. Gascón-Garrido, N. Mainusch, H. Militz et al., "Effects of copper-plasma deposition on weathering properties of wood surfaces," Applied Surface Science, vol. 366, pp. 112-119, 2016.

[16] M.-P. G. Laborie and C. E. Frazier, "13C CP/MAS NMR study of a wood/phenol-formaldehyde resin bondline," Journal of Materials Science, vol. 41, no. 18, pp. 6001-6005, 2006. 


\title{
Paper V
}

\section{Morphologic and Chemical Properties of PMMA/ATH Layers with Enhanced Abrasion Resistance realised by Cold Plasma Spraying at Atmospheric Pressure}

\author{
Lena Wallenhorst ${ }^{1,2}$, Roman Rerich ${ }^{1}$, Matej Vovk ${ }^{3}$, Sebastian Dahle ${ }^{4}$, Holger Militz ${ }^{2}$, \\ Gisela Ohms ${ }^{1}$, Wolfgang Viöl ${ }^{1,5}$
}

${ }^{1}$ University of Applied Sciences and Arts, Laboratory of Laser and Plasma Technologies, Von-Ossietzky-Straße 99, 37085 Göttingen, Germany

${ }^{2}$ University of Göttingen, Wood Biology and Wood Products, Faculty of Forest Sciences, Büsgenweg 4, 37075 Göttingen, Germany

${ }^{3}$ University of Ljubljana, Biotechnical Faculty, Jamnikarjeva 101, 1000 Ljubljana, Slovenia

${ }^{4}$ Clausthal University of Technology, Institute of Energy Research and Physical Technologies, Leibnizstraße 4, 38678 Clausthal-Zellerfeld, Germany

${ }^{5}$ Fraunhofer Institute for Surface Engineering and Thin Films, Application Center for Plasma and Photonics, Von-Ossietzky-Str. 100, 37085 Göttingen, Germany

\begin{abstract}
This study investigated the morphologic and chemical properties of coatings based on PMMA/ATH powder and deposited by cold plasma spraying on wood and glass. Since the deposition of pure PMMA/ATH powder with air as process gas yielded coatings with insufficient abrasion resistance, two modifications of the basic process were investigated. Previous studies showed that replacing air as process gas with forming gas did not enhance the abrasion resistance, but the addition of a phenolformaldehyde resin $(\mathrm{PF})$ succeeded in stabilising the particle coatings. In this work, results from morphologic and chemical analysis suggested an encasement of the PMMA/ATH particles by plasmamodified PF and thus a fusion of individual particles, explaining the enhanced bonding. Moreover, adhesion tests confirmed an outstanding bonding between the coating and wood as well as glass, which is assumed to result from interactions between the PF's hydroxyl groups and functional groups on the substrates' surfaces. Studies on the wettability revealed a hydrophobic character of such coatings, therefore generally indicating a possible application for example, to reduce water uptake by wooden materials.
\end{abstract}

Keywords: Plasma Coatings, PMMA/ATH, PF, Adhesion, XPS, Recycling/Reuse

\section{Introduction}

Poly(methyl methacrylate), PMMA, that is reinforced with aluminium trihydrate $\left(\mathrm{Al}(\mathrm{OH})_{3}\right.$ or $\left.\mathrm{ATH}\right)$ and other additives for achieving specific properties is classified as solid surface material [1], with characteristics meeting the requirements of the performance standard ANSI/ICPA SS-1-2001. The PMMA/ATH composite, as it is abbreviated, was developed and described by Duggins and coworkers [2,3]. In general, the composites contain about 50 to $70 \mathrm{wt} \%$ of ATH filler and about 30 to 50 wt. \% of PMMA resin. Because of this composition, machining of PMMA/ATH composite is very similar to machining of wood, but PMMA/ATH can additionally be thermoformed [4-7], similar to thermoplastics. Unlike wood, though, it exhibits a high moisture resistance along with both a good chemical and UV resistance. These properties make PMMA/ATH composites widely useable as working surfaces and claddings for indoor and outdoor applications. Because of the possibility to gain antimicrobial characteristics [8], this material is also of use in laboratories, hospitals and public places. 
The production of workpieces, however, includes trimming and sanding of the PMMA/ATH sheets, leading to about $20 \%$ of the overall material produced becoming post-industrial waste. These cut-offs and waste powder amount to approximately 1000 tons of PMMA/ATH waste every year in Slovenia alone. Different methods of recycling this particular waste have already been considered [9-15], but none of them has yet provided a simple, sustainable and cost-effective solution. In the past few years, some research has aimed to develop new methods of recycling or re-using PMMA/ATH waste and byproducts [16-19]. The common idea throughout this research was to connect the PMMA/ATH with wood, potentially leading to improved properties of so-called wood-plastic composites, or the acquisition of a new composite system that combines PMMA/ATH and wood. It has been found that the adhesives most commonly used in wood industry (urea-formaldehyde (UF), melamine ureaformaldehyde (MUF) and phenol-formaldehyde (PF)) can provide good bond strength between PMMA/ATH and wood [16]. In further work [13], PMMA/ATH powder was deposited on wood by cold plasma spraying using air as process and carrier gas. A functionalisation of the used PMMA/ATH material could be detected; however, it did not yield abrasion resistant coatings. Therefore, the use as protective coatings on wood was not possible. In the present study, the replacement of air by forming gas was investigated; a factor which could yield more reactive molecule fragments and consequently improve the adhesion.

Moreover, our preliminary research suggested that PMMA/ATH boards and waste material are compatible with phenol formaldehyde (PF) resin [16], yielding the opportunity to use this resin in the production of a recycled composite. PF resins are most often used in wood composite adhesives [20]. Powdered novolac PF can easily be combined with the PMMA/ATH powder for application by atmospheric cold plasma spraying. The use of a powdered phenol novolac resin with a medium hexamethylenetetramine (HMTA) content and medium flow turned out to be appropriate for the intended application. This kind of resin was intentionally formulated as a binding agent for the manufacturing of hot or highly compressed cold-pressed grinding wheels, where high thermal resistance and toughness is required. The resin used in this study melts at about $90^{\circ} \mathrm{C}$ and reacts at $130{ }^{\circ} \mathrm{C}$. When cured, it has high dry and wet strength and is very resistant to water and damp atmospheres [20].

In previous studies on the abrasion resistance of PMMA/ATH-based coatings [21], the replacement of air as process and carrier gas did not yield any improvement regarding particle fixation. The addition of PF resin to the PMMA/ATH base material, however, successfully stabilised the coatings. Therefore, this method provides a promising approach to recycling an industrial waste in consideration of the material's protective potential. Based on these previous results, the present research investigated morphologic and chemical properties as well as wettability of the resulting coatings to elucidate possible mechanisms that explain the outstanding adhesion achievable by the addition of PF and the failure of stabilisation by using forming gas. Moreover, adhesion tests were carried out to complement the previous studies on abrasion resistance.

\section{Materials and Methods}

\section{Cold plasma spraying}

The coatings were created by use of a setup as described previously [13,21-23]. In short, the powder material was injected into the effluent plasma of a jet discharge generated by the application of a pulsed high voltage (ignition voltage: approx. $15 \mathrm{kV}$, effective voltage: $2-3 \mathrm{kV}$, max. input power: $2 \mathrm{~kW}$, pulse duration: $5-10 \mu \mathrm{s}$, pulse repetition rate: $50 \mathrm{kHz}$ ). The particle aerosol was obtained by homogeneously compressing dry powder in a cylindrical container and moving it against a rotating brush at a constant velocity defining the feed rate. The carrier gas was streamed above the brush to take up the powder and ensure its transport to the plasma source. To enable a coating of the entire surface, the samples were mounted on an xy-linear stage below the spraying nozzle. As in [21], two different modifications of this basic deposition process were investigated:

A. use of pure PMMA/ATH powder with compressed air as process and carrier gas,

B. use of forming gas instead of compressed air as process and carrier gas,

C. addition of $10 \%$ phenol-formaldehyde powder (PF, Borofen BL-35 from Kolpa d. d.) to the PMMA/ATH powder with compressed air as process and carrier gas.

Coatings were generated on beech wood (Fagus sylvatica L., $4 \times 76 \times 26 \mathrm{~mm}^{3}$ ) and soda-lime glass (microscope slides) as a model substrate for surfaces that cannot be coated easily. Hereafter, wood and 
glass substrates are indicated with the coatings $\mathrm{A}, \mathrm{B}$ and $\mathrm{C}$, using indices $\mathrm{w}$ and $\mathrm{g}$, respectively. Applied deposition parameters are listed in Table 1. These parameters had to be adjusted separately for each coating material and process gas to avoid thermal damage to the wood samples and to concurrently ensure a homogeneous deposition.

Table 1: Summary of coating parameters (subscript w stands for wood, g for glass) [21].

\begin{tabular}{lcccccc}
\hline $\begin{array}{l}\text { Sample } \\
\text { No. }\end{array}$ & $\begin{array}{c}\text { Deposited } \\
\text { material }\end{array}$ & $\begin{array}{c}\text { Process/carrier } \\
\text { gas } \\
\text { (flow rate) }\end{array}$ & $\begin{array}{c}\text { Carrier } \\
\text { gas } \\
\text { pressure } \\
\text { in } \mathrm{MPa}\end{array}$ & $\begin{array}{c}\text { Working } \\
\text { distance } \\
\text { in } \mathrm{mm}\end{array}$ & $\begin{array}{c}\text { Powder } \\
\text { feed rate } \\
\text { in } \mathrm{cm}^{3} / \mathrm{h}\end{array}$ & $\begin{array}{c}\text { Substrate } \\
\text { displacement } \\
\text { in } \mathrm{mm} / \mathrm{s}\end{array}$ \\
\hline $\mathrm{A}_{\mathrm{w} / \mathrm{g}}$ & PMMA/ATH & $\begin{array}{c}\text { Compressed air } \\
(40 \mathrm{~L} / \mathrm{min})\end{array}$ & 0.15 & 13 & 72 & 20 \\
$\mathrm{~B}_{\mathrm{w} / \mathrm{g}}$ & PMMA/ATH & $\begin{array}{c}\text { Forming gas } \\
(35 \mathrm{~L} / \mathrm{min})\end{array}$ & 0.1 & 25 & 121 & 100 \\
$\mathrm{C}_{\mathrm{w} / \mathrm{g}}$ & $\begin{array}{c}\text { PMMA/ATH } \\
(90 \%)\end{array}$ & $\begin{array}{c}\text { Compressed air } \\
(40 \mathrm{~L} / \mathrm{min})\end{array}$ & 0.16 & 16 & 121 & 20 \\
\hline $\mathrm{PF}(10 \%)$ & & & & & \\
\hline
\end{tabular}

Adhesion tests

To investigate the adhesion of the different coatings, modified cross-cut tests were performed (based on DIN ISO 2409 [24]). For this purpose, a lattice of six cuts vertically and horizontally was prepared with a distance of $2 \mathrm{~mm}$ between parallel cuts. After carefully removing loose particles with compressed air, adhesive tape was applied and removed manually. For coated wood and glass, two replicates were prepared for each type of coating.

Layer characterisation

Morphologic properties were studied by scanning electron microscopy (SEM) using a JSM-5600LV from Jeol USA Inc. at an accelerating voltage of $12 \mathrm{kV}$.

Surface sensitive chemical analysis of coated wood was carried out by use of X-ray photoelectron spectroscopy (XPS, PHI 5000 Versa Probe II from ULVAC-PHI). At room temperature and with a base pressure of $5 \times 10^{-8} \mathrm{~Pa}$, a spot of $200 \mu \mathrm{m}$ in diameter was irradiated using a monochromatic $\mathrm{Al} \mathrm{K}$ source $\left(1486.8 \mathrm{eV}\right.$ with $0.45 \mathrm{eV}$ FWHM Ag3d $\left.\mathrm{d}_{5 / 2}\right)$. The pass energy was set to $187.85 \mathrm{eV}$ and $23.5 \mathrm{eV}$ for survey and detail spectra, respectively. For each powder or type of coating, three survey and detail spectra were acquired. Charging of the non-conductive samples was minimised by applying the system's neutralisation system. Data processing was performed using the software MultiPak from PHI [25]. Binding energies are given with respect to the Fermi level and shifted to give an energy of $284.8 \mathrm{eV}$ for the main $\mathrm{C} 1 \mathrm{~s}$ subpeak (corresponding mainly to contributions from $\mathrm{C}-\mathrm{H}, \mathrm{C}-\mathrm{C}$ and $\mathrm{C}=\mathrm{C}$ ). For quantitative analysis and spectral deconvolution, the background (Shirley-type) was subtracted and symmetric Gauss-Lorentz profiles were fitted to the peaks. To account for both measurement and fitting errors, a total error of $5 \%$ was assumed for all stoichiometric results.

In addition to XPS measurements, Fourier Transform Infrared Analysis (FTIR) was performed using a Frontier from PerkinElmer Inc. to study the functional groups involved in the powders and coatings. For this purpose, the coatings were removed from the glass substrates and mixed with $\mathrm{KBr}$ to form a pressed pellet. Spectra were acquired at a resolution of $4 \mathrm{~cm}^{-1}$ in the range of $4000-400 \mathrm{~cm}^{-1}$, each measurement representing the average of 32 spectra. For each system, three different tablets were prepared and analysed.

The wettability of the different coatings on wood was investigated by water contact angle measurements (measuring system G10 from Krüss $\mathrm{GmbH}$ ). To do this, droplets of distilled water $(11 \mu \mathrm{L})$ were applied to the different surfaces and recorded for $10 \mathrm{~s}(25$ frames/sec). Water contact angles were evaluated for each frame. On every coating, five measurements were carried out. 


\section{Results and Discussion}

\section{Adhesion}

In addition to the abrasion tests carried out in previous studies [21], the adhesion was tested by applying and pulling off adhesive tape after cross cutting the coating. On wood, PMMA/ATH coatings without the addition of PF powder were almost completely removed after the first withdrawal of tape (not shown). In contrast, samples $\mathrm{C}_{\mathrm{w} / \mathrm{g}}$ (addition of PF) showed only little change. However, coating material was attached to the adhesive tape. For the samples coated with PMMA/ATH and PF powder, the tests were therefore extended. It was found that most of the coating could be removed after multiple withdrawals of adhesive tape (see Figure 1(a) and Figure 1(b)). Damage along the grid lines are assumed to result mainly from the cutting procedure. On wood, about 21 withdrawals still removed some coating material. The exact number is assumed to be strongly related to the layer thickness and should therefore only be taken as a rough relative estimate. For further withdrawals, no particles remained on the tape even though part of the coating was still present on the sample. Hence, the adhesion between the individual particles still needs improvement, but the bonding between the coating and the beech substrate already proved to be strong. The good adhesion to the substrate may be explained by secondary interactions between the wood constituents and the resin's hydroxyl groups [26]. These results support the hypothesis that complete removal of the coating on wood during the abrasion tests [21] was at least partly due to wood failure instead of insufficient adhesion between the coating and the substrate.

On glass (sample $\mathrm{C}_{\mathrm{g}}$ ), coating material was also still present on the adhesive tape after several removals ( 7 and 9 , resp.). For additional withdrawals, no coating material could be removed even though large parts were still on the sample. These results are in accordance with the abrasion tests [21], showing that the adhesion on glass was superior even to the adhesion on wood. Except for damage along the grid lines, the sample was still completely covered by the coating. In contrast, coatings without PF could be removed completely from glass substrates after only three withdrawals.

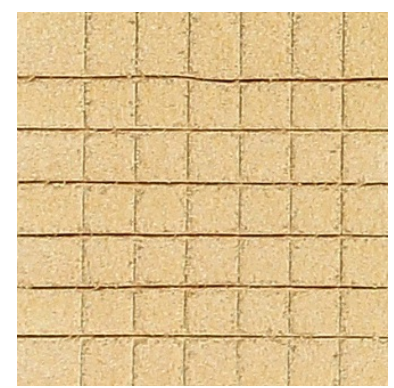

(a)

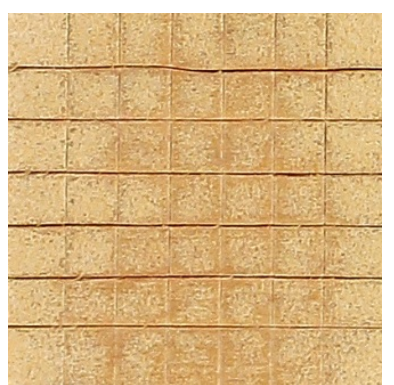

(b)

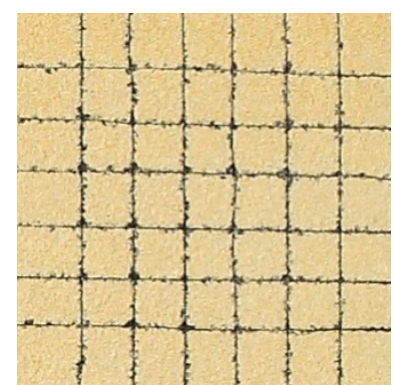

(c)

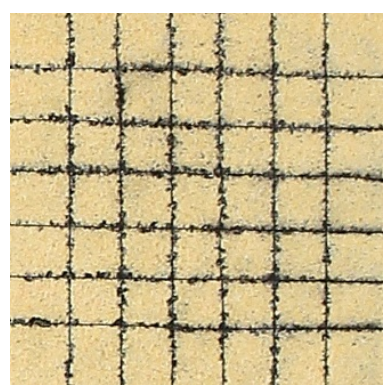

(d)

Figure 1: Cross cut tests on wood and glass coated with PMMA/ATH and PF. (a): coated wood after first removal, (b) coated wood after 22nd removal, (c) coated glass after first removal, (d) coated glass after 10th removals

\section{Morphology}

Figure 2 shows representative SEM images of the different coatings (frame size: $48 \times 64 \mu \mathrm{m}^{2}$ ). Figure 2(a) and Figure 2(b) display the pure PMMA/ATH coatings with air and forming gas as process and carrier gas, respectively. The shape and size of the individual particles did not differ from the untreated powder (not shown). No evidence is visible for film forming processes, therefore explaining the insufficient abrasion resistance and adhesion. In contrast, the coating based on a mixture of PMMA/ATH and PF powder (Figure 2(c)) appeared to feature no individual particles but rather particles that seemed to be fused. Presumably, the PF covered the PMMA/ATH particles and, by melting or reacting in the plasma, it connected the particles and thus led to an improved abrasion resistance and adhesion. This hypothesis was also supported by XPS measurements (see below). However, the SEM measurements also revealed a very rough structure involving large spatial distances between the agglomerates. This large spatial distance together with the ragged shape of the particles may prevent sufficient contact of the individual agglomerates and could therefore explain the insufficient adhesion and abrasion resistance within the coatings. 


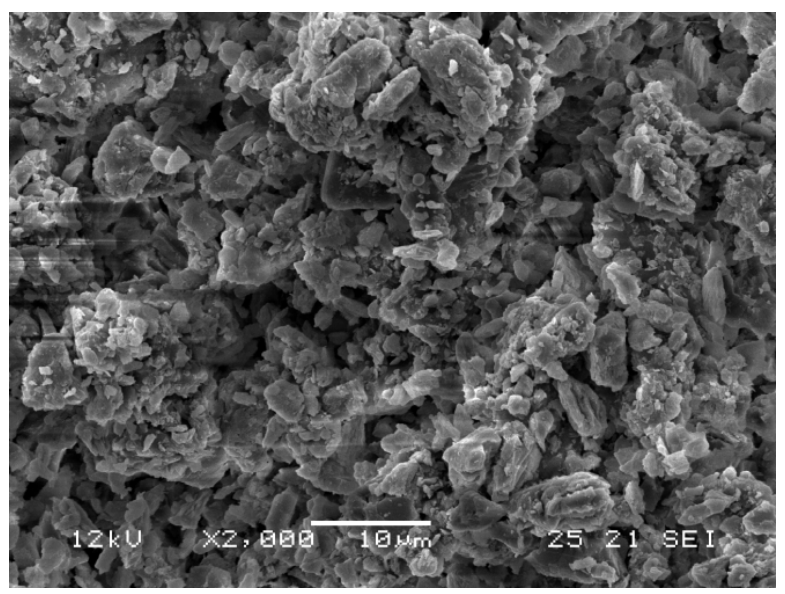

(a)

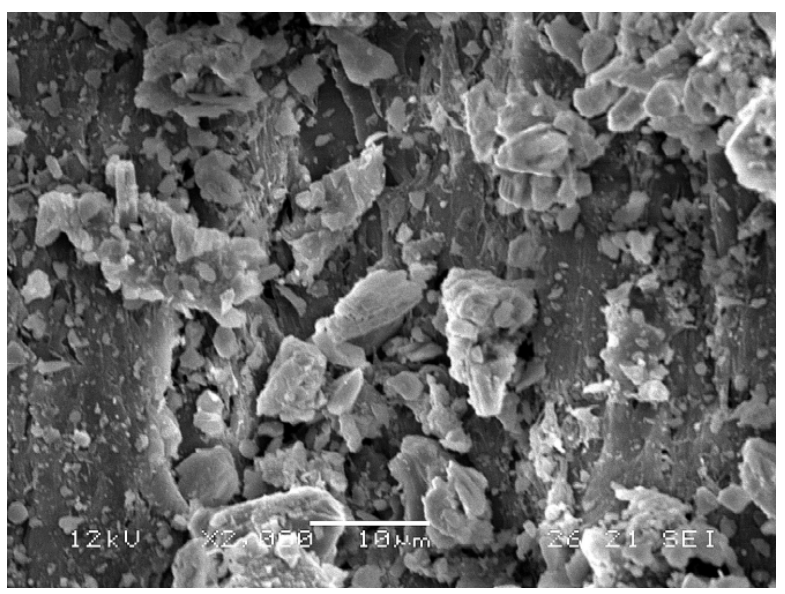

(b)

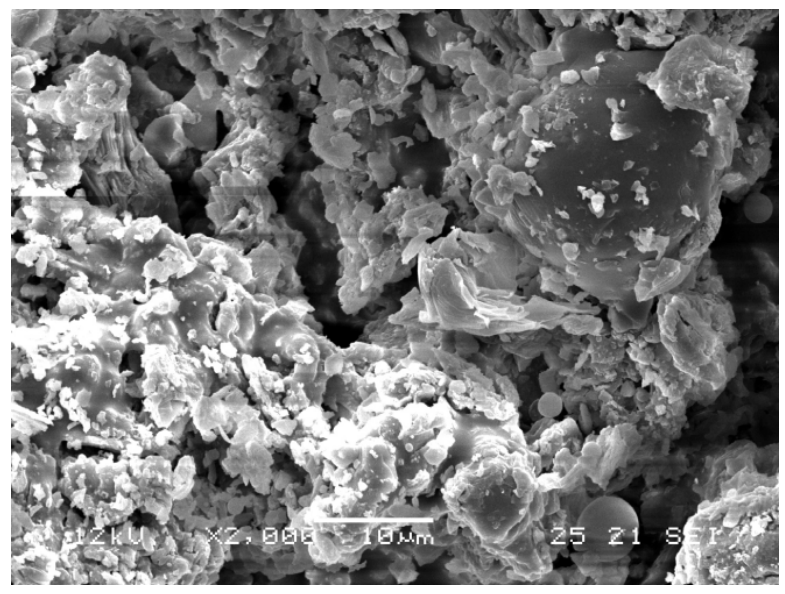

(c)

Figure 2: SEM images of different PMMA/ATH coatings on wood. (a): pure PMMA/ATH with air as process/carrier gas, (b): PMMA/ATH and forming gas as process/carrier gas, and (c): PMMA/ATH and PF powder coating with air as process/carrier gas.

\section{Chemical properties}

XPS survey spectra showed a slight contamination from sodium for some coatings. Since its content did not exceed 1.5 at. \%, the Na contribution was taken into account when determining the chemical composition, but is not listed in this article. The elemental composition of the different systems is summarised in Table 2. 
Table 2: Elemental composition of the different samples as determined by XPS together with the resulting ratio O/C. Mean values of three measurements are displayed together with standard deviations in parentheses. Contributions from Na contamination are not listed.

\begin{tabular}{|c|c|c|c|c|c|}
\hline Sample & $\begin{array}{c}\text { O1s } \\
\text { c in at. } \%\end{array}$ & $\begin{array}{c}\text { C1s } \\
\mathrm{c} \text { in at. } \% \\
\end{array}$ & $\begin{array}{c}\text { Al2p } \\
\mathrm{c} \text { in at. \% }\end{array}$ & $\begin{array}{c}\text { N1s } \\
\text { c in at. \% }\end{array}$ & $\mathbf{O} / \mathrm{C}$ \\
\hline Untreated PMMA/ATH & $\begin{array}{l}49.8 \\
(0.8)\end{array}$ & $\begin{array}{l}38.4 \\
(0.5)\end{array}$ & $\begin{array}{l}11.8 \\
(0.4)\end{array}$ & & 1.3 \\
\hline $\begin{array}{l}\text { Coating } A_{w} \\
\text { (PMMA/ATH in air) }\end{array}$ & $\begin{array}{l}50.9 \\
(0.2)\end{array}$ & $\begin{array}{l}32.1 \\
(0.4)\end{array}$ & $\begin{array}{l}15.6 \\
(0.3)\end{array}$ & & 1.6 \\
\hline $\begin{array}{l}\text { Coating } \mathrm{B}_{\mathrm{w}} \\
\text { (PMMA/ATH in } \mathrm{N}_{2}+\mathrm{H}_{2} \text { ) }\end{array}$ & $\begin{array}{l}47.8 \\
(0.8)\end{array}$ & $\begin{array}{l}37.5 \\
(1.2)\end{array}$ & $\begin{array}{l}13.7 \\
(0.4)\end{array}$ & & 1.3 \\
\hline Untreated PF & $\begin{array}{l}16.1 \\
(0.3)\end{array}$ & $\begin{array}{l}81.8 \\
(0.1)\end{array}$ & & $\begin{array}{c}2.2 \\
(0.2)\end{array}$ & 0.2 \\
\hline $\begin{array}{l}\text { Untreated PF + } \\
\text { PMMA/ATH }\end{array}$ & $\begin{array}{l}42.8 \\
(1.4)\end{array}$ & $\begin{array}{l}46.8 \\
(1.8)\end{array}$ & $\begin{array}{c}9.9 \\
(0.6)\end{array}$ & $\begin{array}{c}0.4 \\
(0.3)\end{array}$ & 0.9 \\
\hline $\begin{array}{l}\text { Coating } \mathrm{C}_{\mathrm{w}} \\
(\mathrm{PF}+\mathrm{PMMA} / \mathrm{ATH} \text { in air) }\end{array}$ & $\begin{array}{l}34.0 \\
(0.4)\end{array}$ & $\begin{array}{l}57.3 \\
(0.6)\end{array}$ & $\begin{array}{c}5.4 \\
(0.2)\end{array}$ & $\begin{array}{c}3.3 \\
(0.3)\end{array}$ & 0.6 \\
\hline
\end{tabular}

The composition of the untreated PMMA/ATH powder differed from previous results reporting an $\mathrm{Al}$ content of 7.2 at. \% [13], presumably because the powders originated from different production lots. When PMMA/ATH powder was deposited using air as carrier and process gas (sample $\mathrm{A}_{\mathrm{w}}$ ), the carbon content decreased while aluminium increased. Therefore, it is reasonable to assume PMMA was etched and thus ATH filler particles were uncovered. Accordingly, the ratio O/C increased. Etching was also observed in [13], but to a higher extent (increase of Al content by a factor of about 2.2), supporting that the deposition parameters (i. e. different process gas flow and particle feed rate in this case) influence the reactions in the plasma. Kuzuya et al. [27] proposed a chain scission mechanism to explain plasma-etching of PMMA. With forming gas as process and carrier gas (sample $\mathrm{B}_{\mathrm{w}}$ ), the increase in aluminium content was less pronounced. However, since the coating parameters had to be varied to avoid thermal damage to the substrates, direct comparison remains speculative.

With the addition of PF powder (10\%) to the PMMA/ATH powder, a strong decrease of aluminium content together with a significant increase in carbon and nitrogen took place. Aluminium indicates the presence of PMMA/ATH, whereas nitrogen occurs in the HMTA contained in the PF powder. Therefore, these results suggest that after deposition, the reacted PF encased the PMMA/ATH particles, which could explain both the fusion of the particles observed with SEM and the enhanced adhesion. Moreover, the nitrogen content of the deposited coatings ( of pure PF powder. This may be explained by the incorporation of nitrogen from the ionised atmosphere during the deposition process [28]. A thorough characterisation of this specific plasma setup is still subject to current and future projects, but preliminary measurements showed a predomination of $\mathrm{NO}$ and $\mathrm{OH}$ species in the plasma, thus supporting the hypothesis of nitrogen incorporation. In addition, the plasma is likely to open the phenolic rings to some extent, thus forming chains of conjugated double bonds. Such reactions are well known to be the result of direct and remote plasma treatments in oxygen containing atmospheres for a variety of polymers containing phenol groups, such as polystyrene, bisphenol A polycarbonate, polyethylene terephthalate, or polyphenylsulfone [29-31]. Due to steric effects, this modified structure might acquire a higher density and therefore, the HMTA could be overrepresented on the surface.

To further study the changes induced by the plasma treatments, the $\mathrm{C} 1 \mathrm{~s}$ peak was decomposed into its different contributions. The different untreated materials already feature a complex carbonic structure, 
leading to various overlapping peaks, and the different plasma treatments induced further major changes. Therefore, not every individual bond could be isolated in a reliable way. Instead, peaks covering different regions were fitted to the $\mathrm{C} 1 \mathrm{~s}$. According to the literature [32-38], these regions were defined as follows:

- $\mathrm{C} 1$ : Contributions from $\mathrm{C}-\mathrm{H}$, but also $\mathrm{C}-\mathrm{C}$ and $\mathrm{C}=\mathrm{C}$ bonds (overlap with $\mathrm{C} 2$ ),

- $\quad$ shifted to $\mathrm{E}_{\mathrm{C} 1}=284.8 \mathrm{eV}$,

- $\mathrm{C} 2$ : located at a binding energy of $\mathrm{E}_{\mathrm{C} 1}+(1.3$ to 1.7$) \mathrm{eV}$, mainly accounting for $\mathrm{C}-\mathrm{O}, \mathrm{C}-\mathrm{OH}$, $\mathrm{C}-\mathrm{N}$ and $\mathrm{C}-\mathrm{O}-\mathrm{C}$, but with contributions from $\mathrm{C}-\mathrm{C}$ and $\mathrm{C}=\mathrm{C}$ bonds,

- $\mathrm{C} 3$ : at $\mathrm{E}_{\mathrm{C} 1}+(2.8$ to 3.3) $\mathrm{eV}$, representing $\mathrm{C}=\mathrm{O}$ and $\mathrm{O}-\mathrm{C}-\mathrm{O}$,

- $\mathrm{C} 4:$ at $\mathrm{E}_{\mathrm{C} 1}+(3.8$ to 4.0$) \mathrm{eV}$, corresponding to $\mathrm{O}-\mathrm{C}=\mathrm{O}$.

Moreover, the full width at half maximum (FWHM) was constrained to be the same for all peaks within each deconvolution. Even though this assumption is expected to cause distortion of the results to some extent, the constraint was needed for a thorough fitting procedure, taking into account the variety of carbon bonds that may occur. The quantitative results should thus only be taken as an estimate.

The C1s peaks along with the decomposition for the systems are displayed in Figure 3 for representative spectra and quantitative results are summarised in Table 3. For all samples except the untreated PMMA/ATH powder, mean values of three measurements are listed. One measurement of untreated PMMA/ATH powder led to very different results than the other two and literature values [32], therefore the third measurement was excluded and the values represent the mean of only two measurements. The distortion may be explained by contamination which was likely to occur because the powder represents an industrial waste.

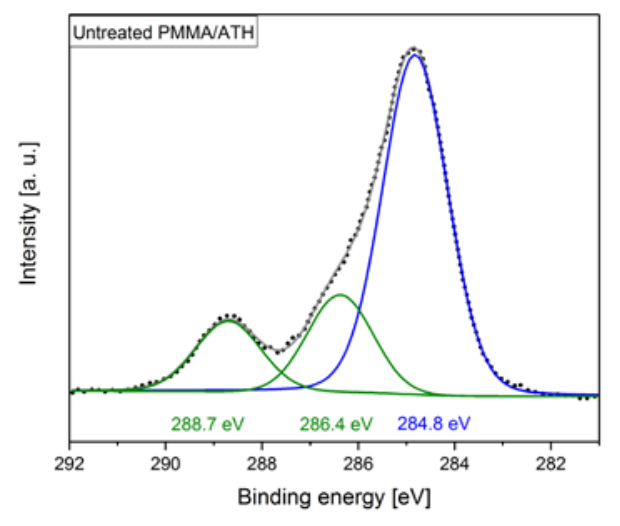

(a)

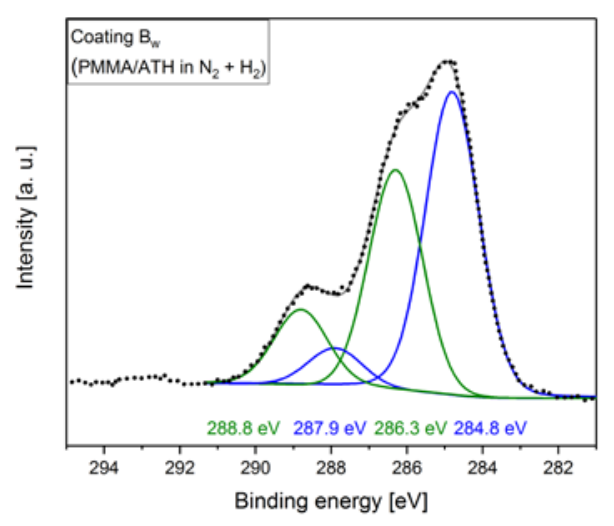

(c)

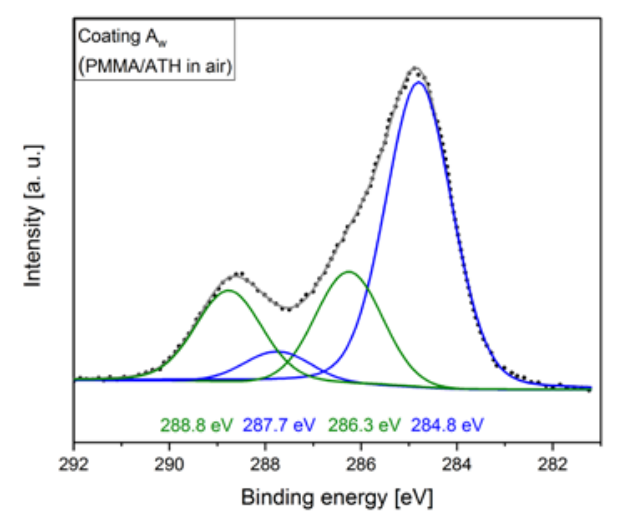

(b)

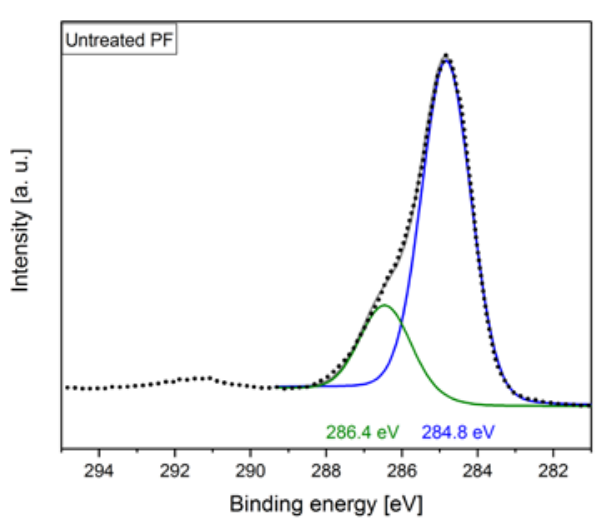

(d) 


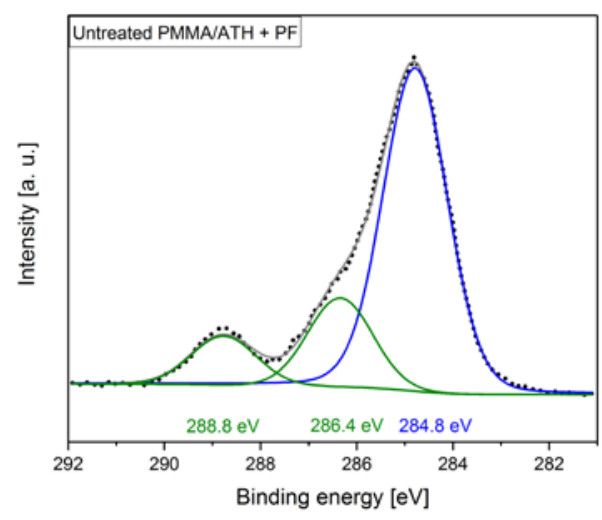

(e)

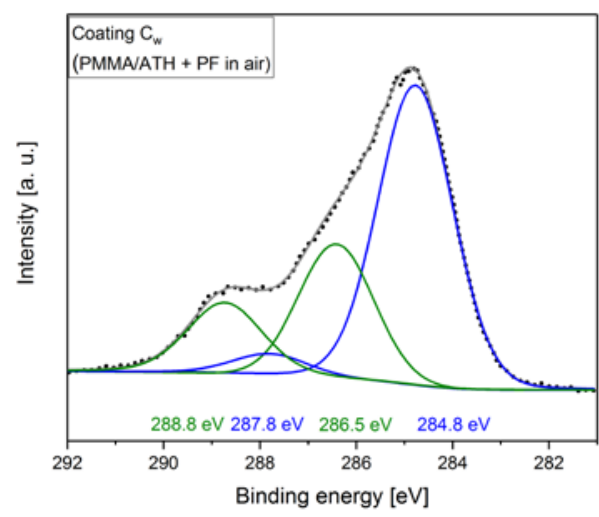

(f)

Figure 3: Representative deconvolutions of the C1s peaks. Solid grey line: cumulative fit, dotted line: measured data. The binding energies of the subpeaks corresponding to regions $\mathrm{C} 1-\mathrm{C} 4$ are depicted below the spectra. Region C1: $\mathrm{C}$ H, C-C, C=C; region C2: C-O, C-OH, C-N, C-O-C, (C-C, C=C); region C3: C=O, O-C-O; region C4: O-C=O.

Table 3: Spectral deconvolution of the C1s peaks. $A_{\text {meas }}$ denotes the measured relative area of the specific carbon bonds within the $\mathrm{C} 1 \mathrm{~s}$. Values in parentheses denote standard deviations. *One measurement of untreated PMMA/ATH powder was probably distorted by contamination; therefore, the values given in this case are mean values of only two measurements.

\begin{tabular}{|c|c|c|c|c|}
\hline Sample & $\begin{array}{c}\text { C1 } \\
\mathrm{C}-\mathrm{H}, \mathrm{C}-\mathrm{C}, \\
\mathrm{C}=\mathrm{C} \\
\mathrm{A}_{\text {meas }} \text { in } \% \\
\end{array}$ & $\begin{array}{c}\text { C2 } \\
\mathrm{C}-\mathrm{O}, \mathrm{C}-\mathrm{OH}, \\
\mathrm{C}-\mathrm{N}, \mathrm{C}-\mathrm{O}-\mathrm{C} \\
(\mathrm{C}-\mathrm{C}, \mathrm{C}=\mathrm{C}) \\
\mathrm{A}_{\text {meas }} \text { in } \% \\
\end{array}$ & $\begin{array}{c}\mathrm{C} 3 \\
\mathrm{C}=\mathrm{O}, \mathrm{O}-\mathrm{C}-\mathrm{O} \\
\mathrm{A}_{\text {meas }} \text { in } \%\end{array}$ & $\begin{array}{c}\text { C4 } \\
\mathrm{O}-\mathrm{C}=\mathrm{O} \\
\mathrm{A}_{\text {meas }} \text { in \% }\end{array}$ \\
\hline Untreated PMMA/ATH* & $\begin{array}{l}67.5 \\
(0.1)\end{array}$ & $\begin{array}{l}18.6 \\
(0.1)\end{array}$ & $\begin{array}{c}0.0 \\
(0.0)\end{array}$ & $\begin{array}{l}13.9 \\
(0.0)\end{array}$ \\
\hline $\begin{array}{l}\text { Coating } A_{w} \\
\text { (PMMA/ATH in air) }\end{array}$ & $\begin{array}{l}60.5 \\
(2.4)\end{array}$ & $\begin{array}{l}18.9 \\
(0.7)\end{array}$ & $\begin{array}{c}4.0 \\
(1.4)\end{array}$ & $\begin{array}{l}16.6 \\
(0.7)\end{array}$ \\
\hline $\begin{array}{l}\text { Coating } \mathrm{B}_{\mathrm{w}} \\
\text { (PMMA/ATH in } \mathrm{N}_{2}+\mathrm{H}_{2} \text { ) }\end{array}$ & $\begin{array}{l}48.7 \\
(1.2)\end{array}$ & $\begin{array}{l}32.3 \\
(1.3)\end{array}$ & $\begin{array}{c}6.5 \\
(0.7)\end{array}$ & $\begin{array}{l}12.5 \\
(0.5)\end{array}$ \\
\hline Untreated PF & $\begin{array}{l}80.9 \\
(0.2)\end{array}$ & $\begin{array}{l}19.1 \\
(0.2)\end{array}$ & $\begin{array}{c}0.0 \\
(0.0)\end{array}$ & $\begin{array}{c}0.0 \\
(0.0)\end{array}$ \\
\hline Untreated PF + PMMA/ATH & $\begin{array}{l}69.9 \\
(1.3)\end{array}$ & $\begin{array}{l}19.3 \\
(0.7)\end{array}$ & $\begin{array}{c}0.0 \\
(0.0)\end{array}$ & $\begin{array}{l}10.8 \\
(0.6)\end{array}$ \\
\hline $\begin{array}{l}\text { Coating } \mathrm{C}_{\mathrm{w}} \\
(\mathrm{PF}+\mathrm{PMMA} / \mathrm{ATH} \text { in air) }\end{array}$ & $\begin{array}{l}58.6 \\
(1.6)\end{array}$ & $\begin{array}{l}24.1 \\
(0.7)\end{array}$ & $\begin{array}{c}3.6 \\
(1.5)\end{array}$ & $\begin{array}{l}13.7 \\
(2.0)\end{array}$ \\
\hline
\end{tabular}

In accordance with previous results [13], the deposition of PMMA/ATH powder with the use of air as process and carrier gas (coating $\mathrm{A}_{\mathrm{w}}$ ) led to a slight oxidation of the PMMA, indicated by a decrease of $\mathrm{C} 1$ for the benefit of $\mathrm{C} 3$ and $\mathrm{C} 4$. In contrast, the use of forming gas instead of air as process and carrier gas seemed to cause a more severe fragmentation and recombination, presumably involving high loss of hydrogen side-chains $\left(\mathrm{CH}_{2}, \mathrm{CH}_{3}\right)$ as supported by the decreasing $\mathrm{Cl}$ peak. The increase in $\mathrm{C} 2$ may partly be caused by fragmentation and subsequent formation of $\mathrm{C}-\mathrm{OH}$ or $\mathrm{C}-\mathrm{O}-\mathrm{C}$ groups. The shake-up around $292.6 \mathrm{eV}$ indicates the generation of $\mathrm{C}=\mathrm{C}$ or other unsaturated bonds. Even though the elemental composition indicated a slight reduction of the PMMA, the oxidative compounds within 
the $\mathrm{C} 1 \mathrm{~s}$ peak seemed to increase. This is assumed to result from an overlap of the regions, particularly $\mathrm{C} 1$ and $\mathrm{C} 2$ which cover various different compounds. Presumably, a strong loss of hydrogencontaining side-chains along with an increase in $\mathrm{C}-\mathrm{C}$ (relative) and $\mathrm{C}=\mathrm{C}$ bonds distorted the results since the $\mathrm{C}-\mathrm{H}$ and $\mathrm{C}-\mathrm{C} / \mathrm{C}=\mathrm{C}$ bonds were summarised as one region despite the difference in binding energy of about $0.72 \mathrm{eV}$ [32]. Nevertheless, the formation of new $\mathrm{C}=\mathrm{O}$ bonds was strongly supported by the data and evidence for severe fragmentation was found. These results may be explained by depolymerisation of PMMA in the plasma through chain scission and depletion of methyl/methylene groups.

The deposition of PMMA/ATH and PF powder with air as process/carrier gas seemed to cause oxidation. Since the results from the elemental composition on the surface and SEM measurements indicated a predominant concentration of PF rather than PMMA on the surface, the oxidation is assumed to affect mainly the resin instead of PMMA during deposition. However, complementary analysis would be needed to elucidate the processes involved.

Since the information depth of XPS usually is well below $10 \mathrm{~nm}$, these measurements cannot be used to study the adhesion mechanism between the coating and the substrates directly. However, the results did suggest that PF underwent distinct chemical changes during the plasma treatment. On wood, the PF's hydroxyl groups are assumed to interact with the wood constituents and ensure bonding [26]. On the surface of $\mathrm{SiO}_{2}$, hydroxyl groups usually play an important role as well [39]. Since plasma treatments involving oxygen species are known to activate surfaces $[38,40]$, the $\mathrm{C}-\mathrm{OH}$ groups of the $\mathrm{PF}$ could react with the Si-OH bond of the glass surface in the presence of an activating plasma, leading to the release of $\mathrm{H}_{2} \mathrm{O}$ and the formation of covalent $\mathrm{C}-\mathrm{O}-\mathrm{Si}$ bonds on the interface. Since even in non-pure $\mathrm{SiO}_{2}$ glass, the density of such functional groups is assumed to be higher on $\mathrm{SiO}_{2}$ than on wood, this could explain the outstanding adhesion on glass compared to wood.

In addition to XPS measurements, infrared spectra of the coatings were acquired by scraping off the layer, mixing it with $\mathrm{KBr}$ and forming a pressed pellet. These transmittance spectra were recorded because the high surface roughness which prevented close contact with the ATR crystal made ATRFTIR measurements impossible. Figure 4 shows representative spectra of untreated PMMA/ATH powder, the untreated mixture of PMMA/ATH and PF powder, as well as the deposited coating $\mathrm{C}_{\mathrm{g}}$. In the spectrum of PMMA/ATH powder, the hydroxyl groups from ATH mainly manifest in the region between $3700-3200 \mathrm{~cm}^{-1}$, around $1021 \mathrm{~cm}^{-1}$, and between approximately $900-459 \mathrm{~cm}^{-1}$ [41]. Moreover, PMMA predominantly appears around $2998 \mathrm{~cm}^{-1}, 2952 \mathrm{~cm}^{-1}, 1487 \mathrm{~cm}^{-1}, 1452 \mathrm{~cm}^{-1}$, $1390 \mathrm{~cm}^{-1}\left(\mathrm{CH}_{2}, \mathrm{CH}_{3}\right)$, and $1732 \mathrm{~cm}^{-1}(\mathrm{C}=\mathrm{O})$ [42]. The addition of PF powder led to the appearance of new peaks which were much more pronounced for the deposited coating. For the coating, the hydroxyl region between $3700-3200 \mathrm{~cm}^{-1}$ was strongly broadened towards lower wavenumbers, resulting from the PF's C-OH bonds. Moreover, phenolic C $=\mathrm{C}$ manifested at $1612 \mathrm{~cm}^{-1}, 1595 \mathrm{~cm}^{-1}$ and $1513 \mathrm{~cm}^{-1}$. The band around $1240 \mathrm{~cm}^{-1}$ may be assigned to $\mathrm{C}-\mathrm{O}$ or $\mathrm{C}-\mathrm{OH}[43,44]$. Since the FTIR spectra include information from the coating's bulk material, they confirm a strong dominance by PF not only on the surface. This dominance may result from both an encasement of the PMMA/ATH particles and an etching of PMMA during the deposition. 


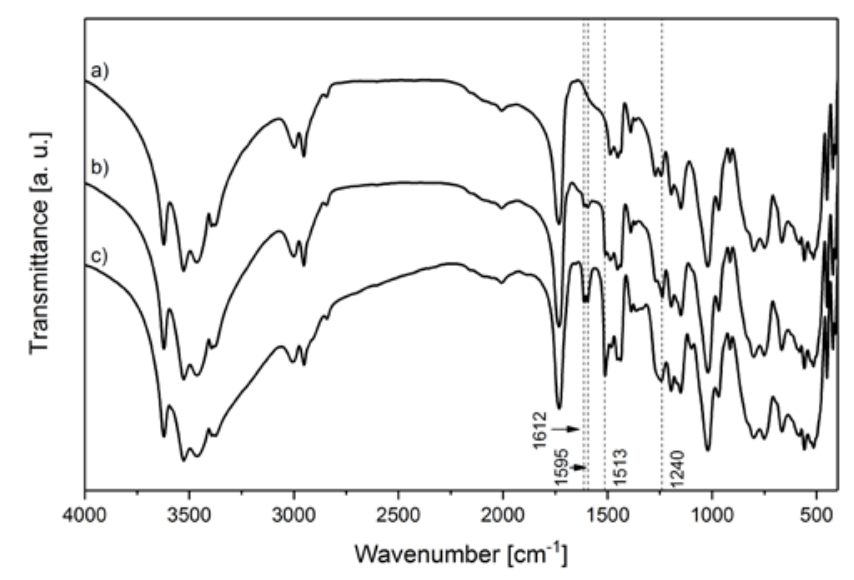

Figure 4: IR-spectra of untreated PMMA/ATH powder (a), untreated mixed PMMA/ATH and PF powder (b) and coating $\mathrm{C}_{\mathrm{g}}(\mathrm{c})$.

Infrared spectra were also recorded for the other coatings. However, since all chemical moieties changed during the deposition, normalisation was found to be challenging. No reliable additional information could be extracted from those spectra; therefore, they are not presented in this article.

\section{Wettability}

Figure 5 shows the evolution of the water contact angles on the different samples. For each time interval, the mean of five measurements is given. In accordance with [13], the contact angles on coating $\mathrm{A}_{\mathrm{w}}$ (Figure 5(a)) decreased quickly and the droplet was absorbed within $10 \mathrm{~s}$. This behaviour is assumed to result from a combination of the rough surface as well as from the chemical changes, namely the exposure of ATH and thus of hydroxyl groups and the oxidation of PMMA. Sample $\mathrm{B}_{\mathrm{w}}$ (Figure 5(b)) appeared to be slightly less hydrophilic, presumably because of less intense etching of PMMA and therefore less ATH on the surface. Moreover, it supports the hypothesis that non-polar C$\mathrm{C}$ and $\mathrm{C}=\mathrm{C}$ bonds dominate and that the apparent increase in polar groups observed for XPS measurements (peak C2) was merely an inaccuracy resulting from the strong overlap of the peaks. In contrast, sample $\mathrm{C}_{\mathrm{w}}$ (Figure 5(c)) was found to be hydrophobic (contact angles above $100^{\circ}$ ), which should result from the significantly increased carbon content on the surface and the decreased share of ATH. Therefore, an application of optimised coatings based on PMMA/ATH and PF powder as protective, hydrophobic coatings is conceivable.

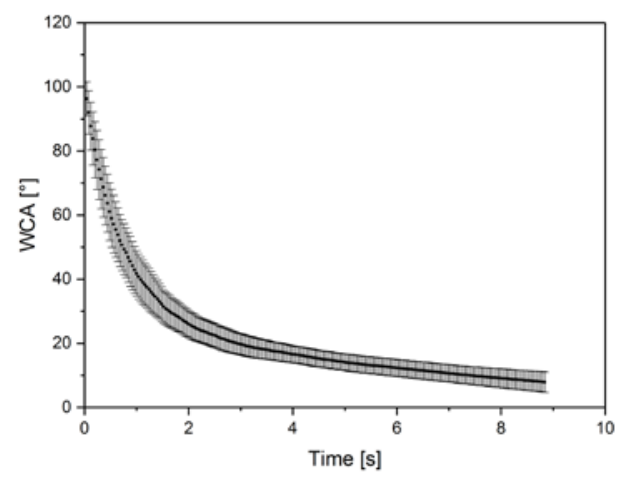

(a)

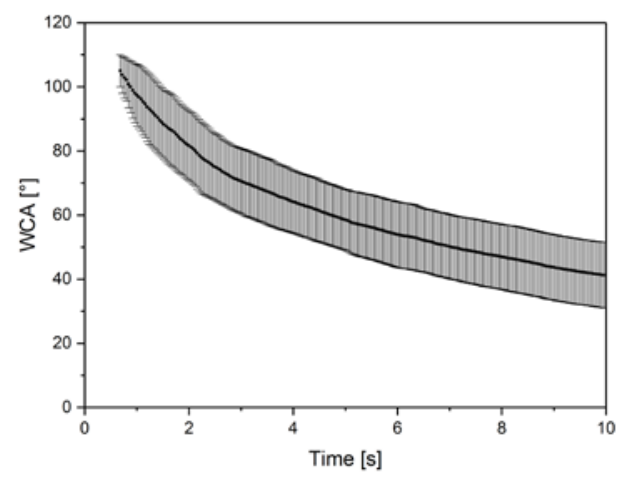

(b) 


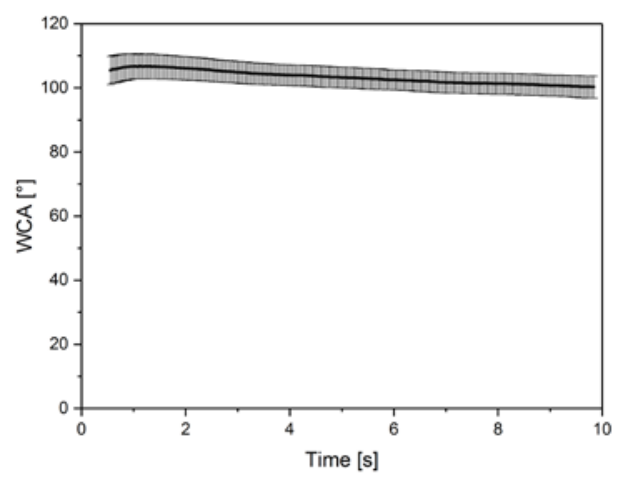

(c)

Figure 5: Evolution of the water contact angle with time. (a): Coating $A_{w}$, (b): Coating $B_{w}$, (c): Coating $C_{w}$.

\section{Conclusion and Outlook}

In this work, cold plasma spraying was used to deposit different kinds of coatings based on PMMA/ATH waste powder to explore possible recycling options as protective layers. The adhesion of the resulting coatings was investigated together with their morphologic and chemical properties to study possible adhesion mechanisms and changes during the plasma treatments.

In an attempt to increase the adhesion of PMMA/ATH coatings to wood and glass substrates, the process and carrier gas was replaced by forming gas. Even though chemical analysis confirmed severe fragmentation and recombination of carbonic compounds, no improvement of the adhesion could be obtained. As for PMMA/ATH coatings deposited with air as process and carrier gas, SEM measurements did not show any evidence for film-forming mechanisms.

In contrast, the addition of a novolac phenol-formaldehyde resin with medium HMTA content significantly enhanced the coating's adhesion, particularly between the coating and the substrate. SEM measurements revealed a fusion of individual particles, but also a very rough surface leading to large spatial distances between larger agglomerations of particles. Therefore, the enhanced but not yet sufficient adhesion between the particles/agglomerates within the coating is assumed to result from a morphologic rather than from a chemical problem. In accordance with the observed fusion of particles on the surface, chemical analysis by XPS and FTIR provided strong evidence for the formation of an envelope around the PMMA/ATH particles made of PF that has reacted in the plasma. In the infrared spectra, a strong increase of PF-related bands was observed, even though only $10 \%$ resin was added to the PMMA/ATH base material. Moreover, XPS measurements revealed an increase of carbon and nitrogen content, supporting a predomination of PF on the surface. Changes in the carbonic structure are therefore assumed to occur mainly in PF. Even though the interface between the coating and the surfaces could not be studied, the reactivity of PF in the plasma supports the hypothesis of interactions between the $\mathrm{C}-\mathrm{OH}$ groups of $\mathrm{PF}$ and $\mathrm{Si}-\mathrm{OH}$ on the glass surface yielding $\mathrm{C}-\mathrm{O}-\mathrm{Si}$ bonds and releasing water following the plasma treatment. The high density of functional groups on glass surfaces may explain the outstanding adhesion between the coating and the substrate. In addition, water contact angle measurements showed hydrophobic properties of the coatings with angles above $100^{\circ}$, therefore generally permitting an application of such coatings for example, to reduce water uptake by wood.

The results suggest that highly adhesive and abrasion-resistant coatings on wood and glass substrates can be achieved by further optimising such coatings generated by cold plasma spraying with mixed PMMA/ATH and PF powder. Since the problems with adhesion within the coatings are assumed to arise from the morphologic and not from the chemical nature, further studies could concentrate on variations of the deposition parameters to reduce piling of particles and agglomerates. For example, the substrate displacement velocity might distinctly impact the resulting structural properties. Moreover, a grinding of PMMA/ATH particles prior to the deposition could contribute to smoother surfaces. Following optimisation, the coatings' protective potential could be explored. Hydrophobic properties were already shown in this work, but the base material features other outstanding properties 
favourable to the protection of wood or other materials (cf. Introduction). Therefore, it could be assessed whether some of these potentially protective properties were transferred to the coatings.

\section{Acknowledgements}

We gratefully acknowledge financial support from the Lower Saxony Ministry of Science and Culture (MWK). The authors also thank Kolpa d.d. for providing the coating materials, Mirco Weber for fruitful discussions about the results, and Roger Skarsten for proofreading the manuscript.

\section{Niedersächsisches Ministerium für Wissenschaft und Kultur}

\section{References}

[1] "Plastics - Decorative solid surfacing materials - Part 2: Determination of properties - Sheet goods," 2007, ISO 19712-2.

[2] R. B. Duggins and C. Ford, US 3847865: Use of alumina trihydrate in a polymethyl methacrylate article, 1974.

[3] R. B. Duggins, C. Ford, H. C. Miller et al., US 3827933: Filled polymethyl methacrylate article and a process for its manufacture, 1974.

[4] P. Gilormini, L. Chevalier, and Régnier, "Modeling the nonlinear PMMA behavior near glass transition temperature: application to its thermoforming," AIP Conference Proceedings, vol. 1315, no. 1, pp. 179-184, 2011.

[5] E. M. Gunel and C. Basaran, "Micro-deformation mechanisms in thermoformed alumina trihydrate reinforced poly(methyl methacrylate)," Materials Science and Engineering: A, vol. 523, no. 1, pp. 160-172, 2009.

[6] R. G. Jagger and A. Okdeh, "Thermoforming polymethyl methacrylate," The Journal of Prosthetic Dentistry, vol. 74, no. 5, pp. 542-545, 1995.

[7] E. Minghetti, US 6562927 B1: Method of making a thermoformable article having uniform distribution of coloring and mineral filler before and after thermoforming, 2003.

[8] S. Sabesan, US 8092815 B2: Antimicrobial solid surface materials containing chitosan-metal complexes, 2012.

[9] W. Kaminsky and J. Franck, "Monomer recovery by pyrolysis of poly(methyl methacrylate) (PMMA)," Journal of Analytical and Applied Pyrolysis, vol. 19, pp. 311-318, 1991.

[10] W. Kaminsky, M. Predel, and A. Sadiki, "Feedstock recycling of polymers by pyrolysis in a fluidised bed," Polymer Degradation and Stability, vol. 85, no. 3, pp. 1045-1050, 2004.

[11] J. Hochberg and R. B. Young, US 4759774 A: Process for cleaning substrates, 1988.

[12] E. Šušteršič, M. Tušar, and A. Zupančič Valant, "Rheological and mechanical characterization of waste PMMA/ATH modified bitumen," Construction and Building Materials, vol. 38, pp. 119$125,2013$.

[13] L. M. Wallenhorst, S. Dahle, M. Vovk et al., "Characterisation of PMMA/ATH layers realised by means of atmospheric pressure plasma powder deposition," Advances in Condensed Matter Physics, vol. 2015, no. 3, pp. 1-12, 2015.

[14] M. Tušar, E. Šušteršič, and A. Zupančičc-Valant, "Alternative PMB produced from recycling waste PMMA/ATH," Transport Problems, vol. 9, no. 2, pp. 5-9, 2014.

[15] M. Tušar, A. Beličič, M. Prešeren et al., EP 20130730823: Modified bitumen and its use for preparing asphalt mixtures and bituminous products, 2015.

[16] M. Vovk and M. Šernek, "Lepljenje lesa in Kerrocka," Pedagoška obzorja 2, vol. 2015, no. 4.

[17] M. Vovk, A. Beličič, and M. Šernek, "Združljivost lesa s kompozitom iz polimetilmetakrilata in aluminijevega hidroksida (Compatibility of wood with a composite from polymethyl methacrylate and aluminium hydroxide)," Znanstveno Srečanje Gozd in Les, pp. 11-13, 2014.

[18] M. Vovk, L. Wallenhorst, C. Kaldun et al., "Air plasma treatment of aluminium trihydrate filled poly(methyl methacrylate)," Submitted to: Journal of Adhesion Science and Technology. 
[19] M. Vovk, M. Šernek, and A. Beličič, SI 24705a: Postopek za pridobivanje kompozitnega materiala iz odpadnih produktov PMMA/ATH kompozita, vezanih z aminoplastičnimi ali fenolnimi lepili, 2015.

[20] United States Department of Agriculture, Wood Handbook: Wood as an Engineering Material, Forest Product Laboratory, USDA, Madison, 2010.

[21] L. Wallenhorst, R. Rerich, M. Vovk et al., "Enhancing the abrasion resistance of PMMA/ATH layers realised by means of atmorpheric pressure plasma powder deposition," Proceedings of the 11th International Conference "Wood Science and Engineering in the third Millenium, 2017.

[22] P. Gascón-Garrido, N. Mainusch, H. Militz et al., "Copper and aluminium deposition by coldplasma spray on wood surfaces: effects on natural weathering behaviour," European Journal of Wood and Wood Products, vol. 75, no. 3, pp. 315-324, 2016.

[23] P. Gascón-Garrido, N. Mainusch, H. Militz et al., "Effects of copper-plasma deposition on weathering properties of wood surfaces," Applied Surface Science, vol. 366, pp. 112-119, 2016.

[24] "Paints and varnishes - Cross-cut tests," 2013, ISO 2409:2013.

[25] MultiPak, Physical Electronics Inc., Chanhassen, USA.

[26] M.-P. G. Laborie and C. E. Frazier, "13C CP/MAS NMR study of a wood/phenol-formaldehyde resin bondline," Journal of Materials Science, vol. 41, no. 18, pp. 6001-6005, 2006.

[27] M. Kuzuya, A. Noguchi, M. Ishikawa et al., "Electron spin resonance study of free-radical formation and its decay of plasma-irradiated poly (methacrylic acid) and its esters," The Journal of Physical Chemistry, vol. 95, no. 6, pp. 2398-2403, 1991.

[28] H. Yasuda and T. Yasuda, "The competitive ablation and polymerization (CAP) principle and the plasma sensitivity of elements in plasma polymerization and treatment," Journal of Polymer Science Part A: Polymer Chemistry, vol. 38, no. 6, pp. 943-953, 2000.

[29] D. Zhang, S. M. Dougal, and M. S. Yeganeh, "Effects of UV irradiation and plasma treatment on a polystyrene surface studied by IR- visible sum frequency generation spectroscopy," Langmuir, vol. 16 , no. 10 , pp. $4528-4532,2000$.

[30] L. J. Gerenser, "XPS studies of in situ plasma-modified polymer surfaces," Journal of Adhesion Science and Technology, vol. 7, no. 10, pp. 1019-1040, 1993.

[31] E. Gonzalez, M. D. Barankin, P. C. Guschl et al., "Ring opening of aromatic polymers by remote atmospheric-pressure plasma," IEEE Transactions on Plasma Science, vol. 37, no. 6, pp. 823$831,2009$.

[32] G. Beamson and D. Briggs, High Resolution XPS of Organic Polymers, Wiley, 1992.

[33] T. Cai, M. Zhou, D. Ren et al., "Highly ordered mesoporous phenol-formaldehyde carbon as supercapacitor electrode material," Journal of Power Sources, vol. 231, pp. 197-202, 2013.

[34] A. Crunteanu, M. Charbonnier, M. Romand et al., "Synthesis and characterization of carbon nitride thin films obtained by laser induced chemical vapour deposition," Surface and Coatings Technology, vol. 125, no. 1, pp. 301-307, 2000.

[35] C. Moreno-Castilla, M. V. López-Ramón, and F. Carrasco-Marın, "Changes in surface chemistry of activated carbons by wet oxidation," Carbon, vol. 38, no. 14, pp. 1995-2001, 2000.

[36] B. P. Payne, M. C. Biesinger, and N. S. McIntyre, "X-ray photoelectron spectroscopy studies of reactions on chromium metal and chromium oxide surfaces," Journal of Electron Spectroscopy and Related Phenomena, vol. 184, 1-2, pp. 29-37, 2011.

[37] M. N. Uddin, O. A. Fouad, M. Yamazato et al., "Deposition and characterization of carbon nitride films from hexamethylenetetramine/ $\mathrm{N}_{2}$ by microwave plasma-enhanced chemical vapor deposition," Applied Surface Science, vol. 240, no. 1, pp. 120-130, 2005.

[38] P. Král, M. Stupavská, J. Šrajer et al., "XPS depth profile of plasma-activated surface of beech wood (Fagus sylvatica) and its impact on polyvinyl acetate tensile shear bond strength," Wood Science and Technology, vol. 49, no. 2, pp. 319-330, 2015.

[39] P. Schönherr, Multiple Oberflächenfunktionalisierung von Mischgläser- und Siliciumdioxidpartikeln als Komponenten für Kompositmaterialien, Dissertation, Technical University Chemnitz, 2013.

[40] E. Kondoh, T. Asano, A. Nakashima et al., "Effect of oxygen plasma exposure of porous spin-onglass films," Journal of Vacuum Science \& Technology B: Microelectronics and Nanometer Structures Processing, Measurement, and Phenomena, vol. 18, no. 3, pp. 1276-1280, 2000. 
[41] J. T. Kloprogge, L. V. Duong, B. J. Wood et al., "XPS study of the major minerals in bauxite: gibbsite, bayerite and (pseudo-)boehmite," Journal of Colloid and Interface Science, vol. 296, no. 2, pp. 572-576, 2006.

[42] H. Nagai, "Infrared spectra of stereoregular polymethyl methacrylate," Journal of Applied Polymer Science, vol. 7, no. 5, pp. 1697-1714, 1963.

[43] R. O. Ebewele, B. H. River, and J. A. Koutsky, "Relationship between phenolic adhesive chemistry and adhesive joint performance: effect of filler type on fraction energy," Journal of Applied Polymer Science, vol. 31, no. 7, pp. 2275-2302, 1986.

[44] G. Socrates, Infrared and Raman Characteristic Group Frequencies: Tables and Charts, John Wiley \& Sons, Chichester, 2001. 